\title{
Co-design Concepts and Subsystem Oriented Development for Academic BSN Platforms
}

A Thesis Presented to

the Faculty of the School of Engineering and Applied Science

University of Virginia

In Partial Fulfillment

of the Requirements for the Degree

Master of Science (Electrical Engineering)

by

Ben Boudaoud

August

2014 


\section{Approval Sheet}

This thesis

is submitted in partial fulfillment of the requirements

for the degree of

Master of Science (Electrical Engineering)

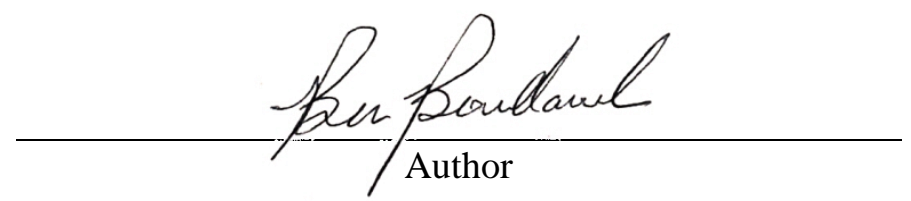

This thesis has been read and approved by the Examining Committee:

\begin{tabular}{c} 
Dr. John Lach \\
\hline Advisor \\
Dr. Harry Powell \\
\hline Dr. John A. Stankovic \\
\hline Dr. Adam T. Barth
\end{tabular}

Accepted for the School of Engineering and Applied Science:

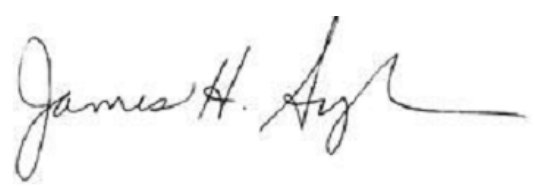

Dean, School of Engineering and Applied Science

August

2014 


\section{Abstract}

Body-worn sensor system design is a promising field of study promoted by emerging interest in longterm, longitudinal monitoring of health-based metrics in the medical and personal fitness contexts. As sensing and reporting modalities diversify, so do the challenges and opportunities faced by traditional embedded designers in developing and producing systems incorporating them. This work aims to significantly alleviate the time-to-prototype and design for novel platform-oriented research utilizing emerging sensing and reporting modalities. The primary contribution is the next generation of the TEMPO core sampling and storage platform in a wearable, expandable, and longitudinally-deployable form-factor. The TEMPO 4 node is designed to serve as a single-board, six or nine degree-of-freedom inertial motion-capture unit as well as an open development platform featuring an easy to interface 16-pin hardware extension port. The general contributions include hardware-firmware-application layer codesign principles and analysis of commercial-off-the-shelf products and protocols for the ultra-low power body-worn sensing context.

This work approaches the issues of hardware-firmware flexibility and robustness using a vertically integrated, iterative design-and-test approach. This approach consists of partitioning the full TEMPO system's operation into conveniently organized, functional subsystems then co-developing firmware libraries on top of iteratively refined hardware platforms. The result of this organization is that design decisions spanning from the application layer to low-level hardware, which may have been traditionally ignored in a more carefully delineated hardware-firmware-software approach, are exposed and examined in the context of modern body sensor node system design. 


\section{Acknowledgements}

This work has been made possible by a number of other researchers, individuals, and organizations who I am proud to be affiliated with.

My graduate research advisor, Dr. John Lach, has provided significant support and promoted continued involvement and innovation in the wearable-space throughout this work. His open mind and open ear have been invaluable throughout the thesis planning, writing, and defense. I would also like to thank Dr. Harry Powell for his technical guidance throughout this work, along with general suggestions for direction throughout my graduate career.

Fellow graduate students Dr. Mark Hanson, Dr. Adam Barth, Sam Ridenhour, and Jeff Brantley have all made contributions that serve as many of the enabling and motivating factors for this work. Without their key contributions and carefeully documented insights much of what is accomplished here would not be possible. I cannot thank them enough for the friendship, knowledge, and mentorship they have provided over the years.

In addition to graduate researchers, undergraduate research assistants played a significant role in many of the development efforts affiliated with this work. Specifically I would like to thank Emilio Esteban, Bill Devine, Davis Blalock, and Anish Simhal for their invaluable contributions to efforts affiliated with background research, platform development, and top-level feedback. Their attitudes, contributions, and dispositions brought excitement to the work and are a stellar example of the capabilities and capacities of the next generation of engineering minds.

I would like to thank the entire INERTIA team for their time and interest throughout the development process. Despite diversified research interests and goals, the continued support for and discussion of platform development, carried out within the group provided a sounding board for future ideas and an active audience for the debate of key concepts.

I would like to thank my family, for always pushing me to succeed and providing support when I did not. In addition I would like to thank my girlfriend, for dealing with me and providing equal parts motivation and all-important distraction throughout this work.

Last, but not least I would like to thank the National Science Foundation ASSIST Engineering Research Center for providing funding and support for my work and tenure at UVa. 


\section{Table of Contents}

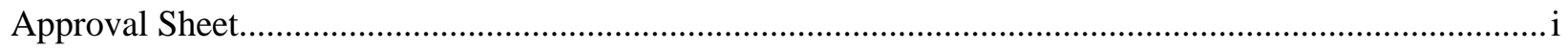

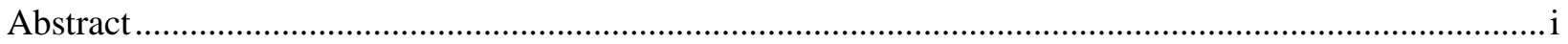

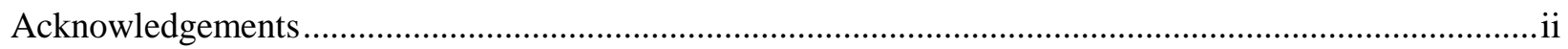

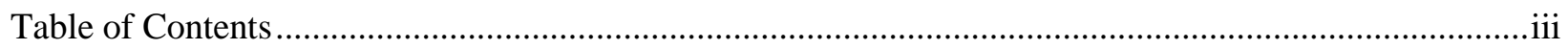

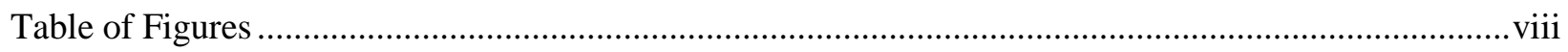

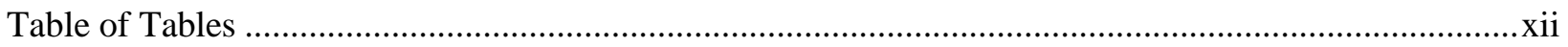

Chapter 1 Introduction and Motivation.............................................................................................

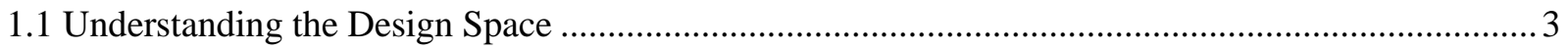

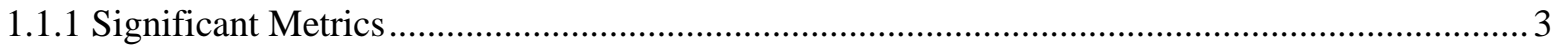

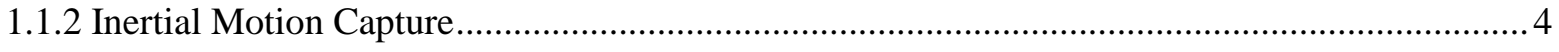

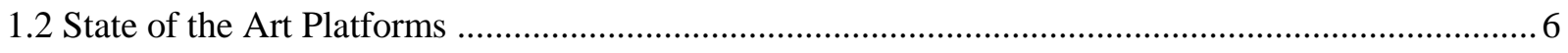

1.3 Motivation and Device Specifications ………………………………………………………….

1.3.1 Deployment Challenges and the TEMPO 3.2 AFO …………………………………….... 8

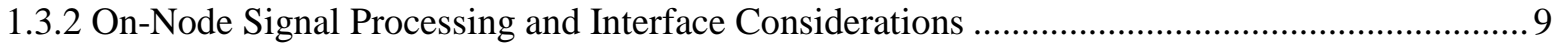

1.3.3 Open Development Considerations ……………………………………………………….... 10

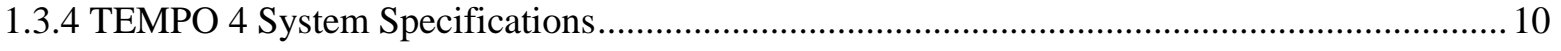

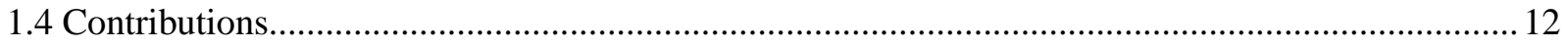

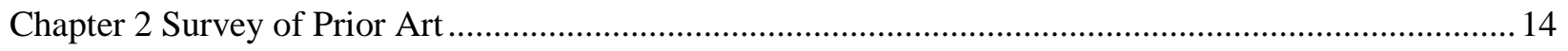

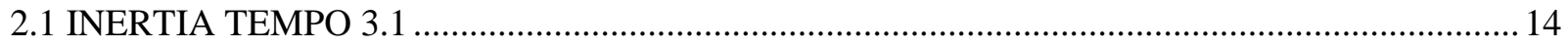

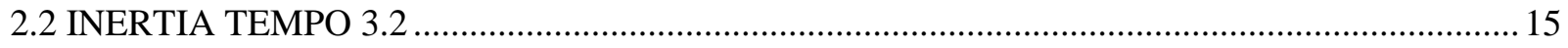

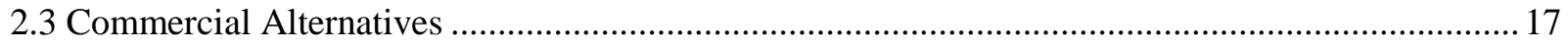

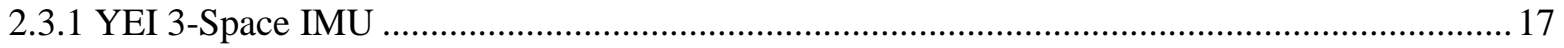

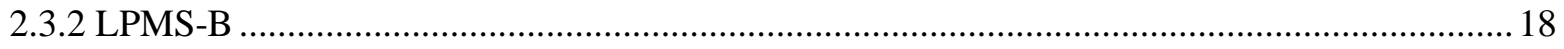

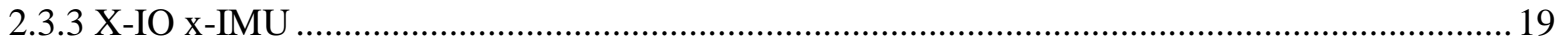




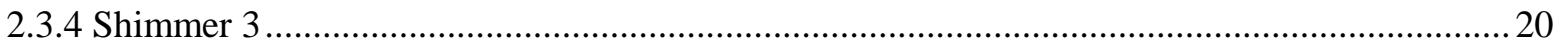

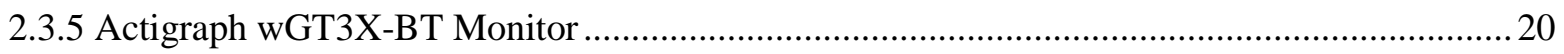

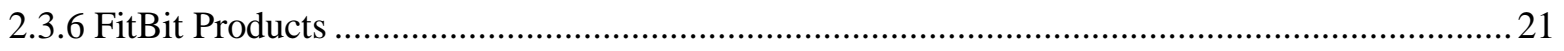

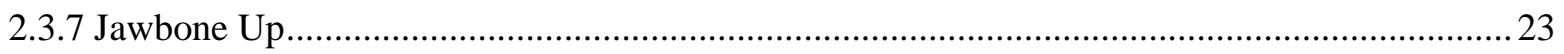

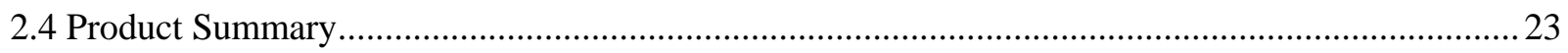

Chapter 3 Co-Design Concepts and Subsystem Designation ...........................................................2 25

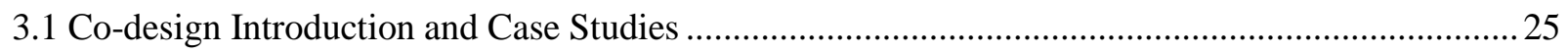

3.1.1 Co-design Case Study 1: USB Transceiver Selection.........................................................25

3.1.2 Co-design Case Study 2: Polled versus Interrupt-driven Operation ........................................29

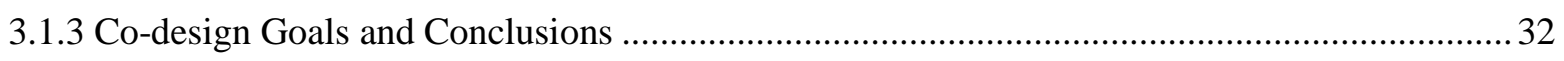

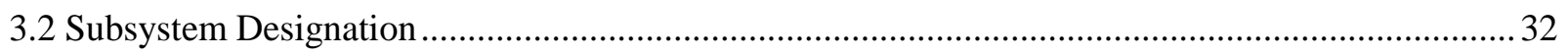

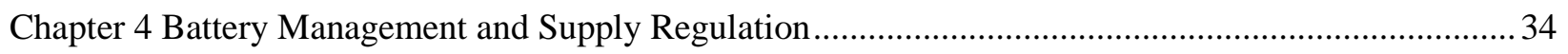

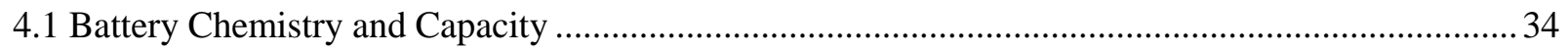

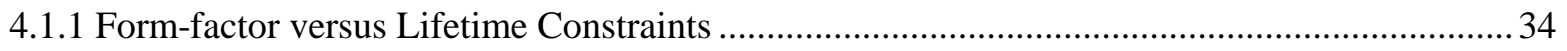

4.1.2 Application Considerations for Lithium Cells ................................................................... 37

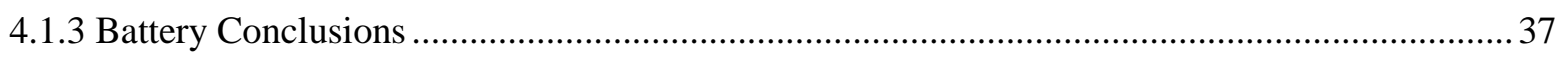

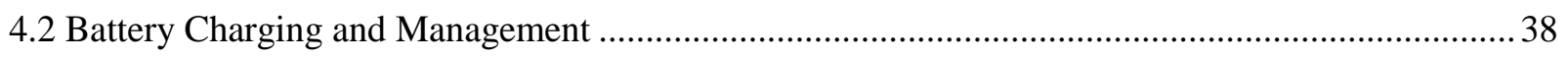

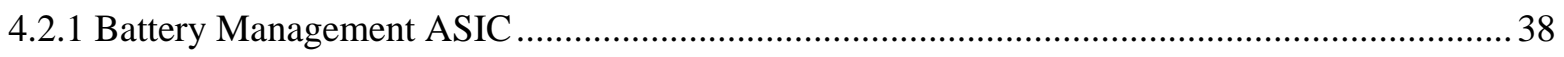

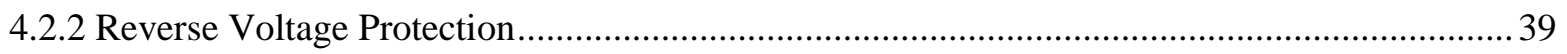

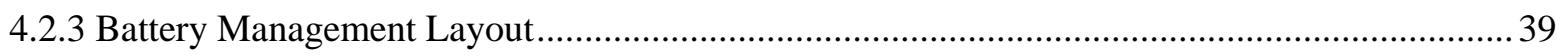

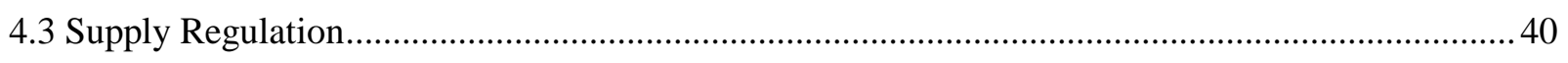

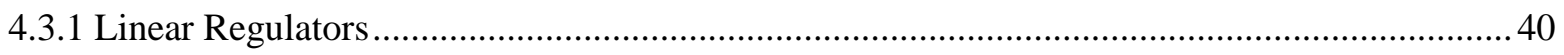

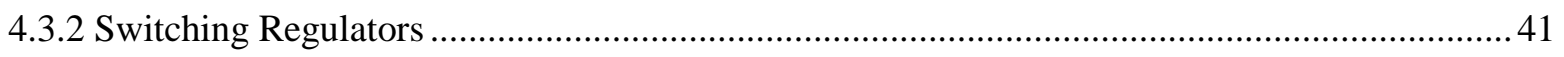

4.3.3 State of the Art Comparison and Regulator Decision .......................................................... 43

4.4 Battery Management and Regulation Summary and Conclusions .............................................. 44

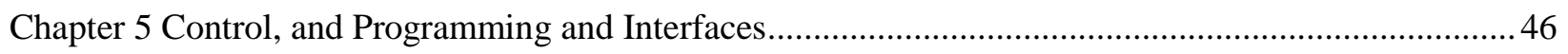




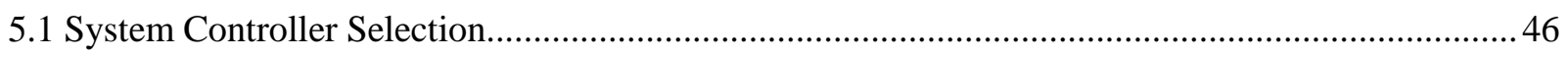

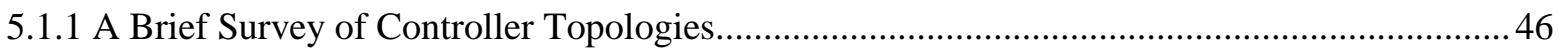

5.1.2 Operating Constraints and MCU Selection....................................................................... 49

5.1.3 MSP430 Family and Device Selection and Prototyping .....................................................50

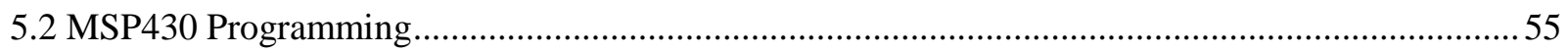

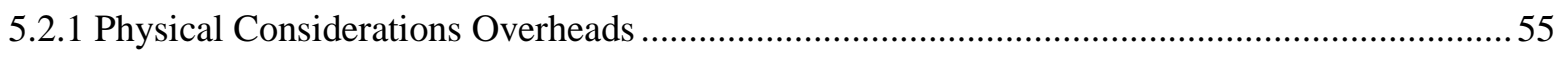

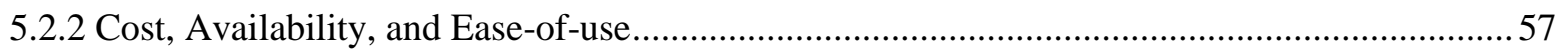

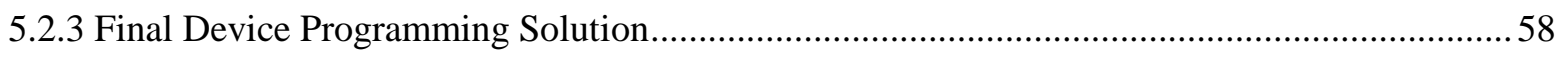

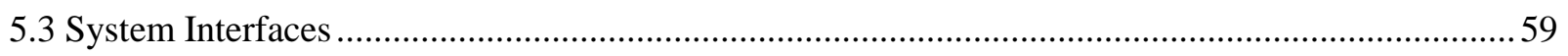

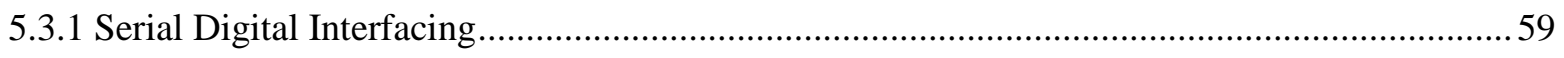

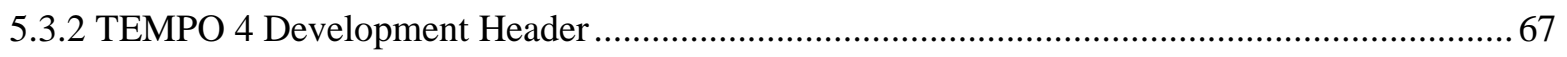

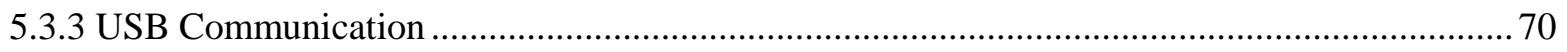

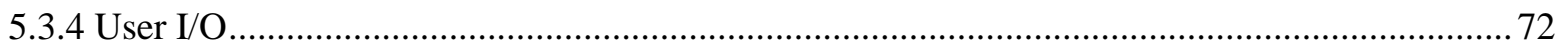

5.4 Control, Programming and Interfaces Summary and Conclusions ............................................ 73

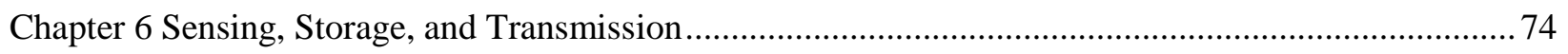

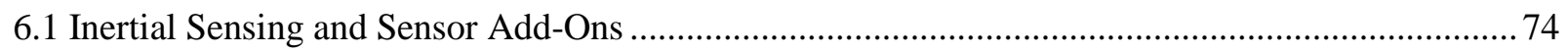

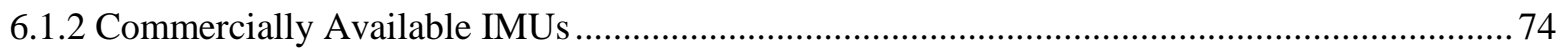

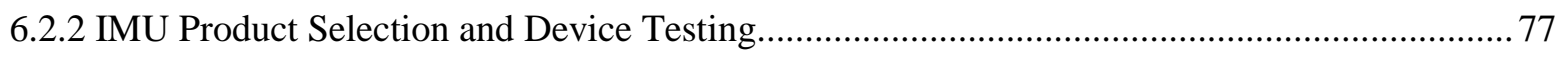

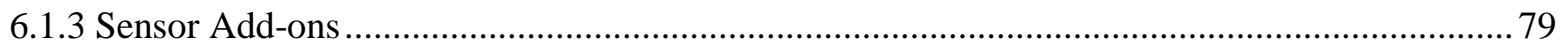

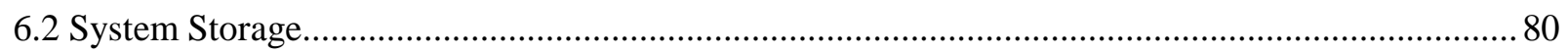

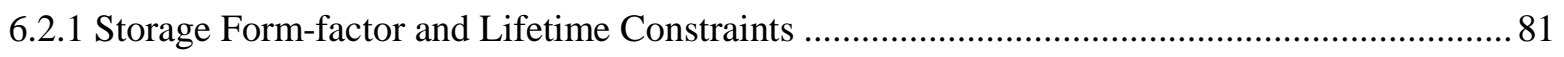

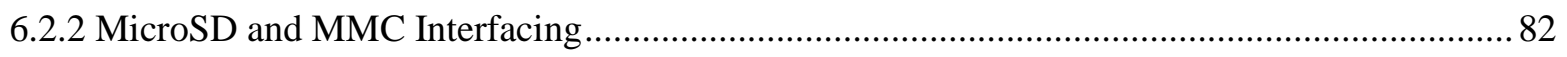

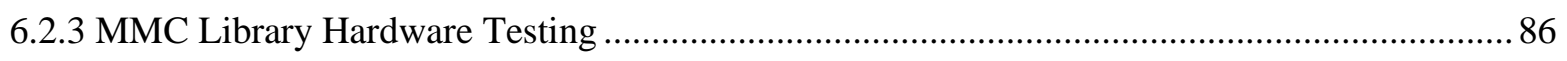

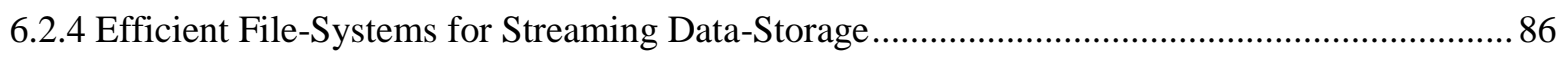

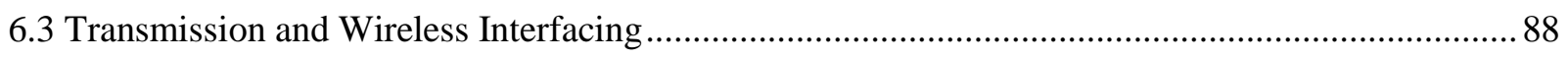

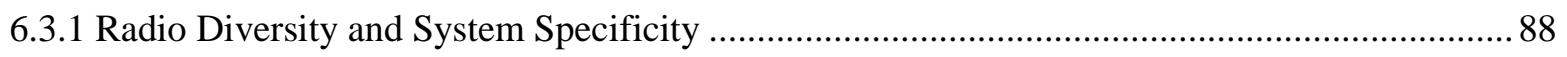


6.3.2 Physical Overhead and RF Design Challenges ............................................................. 91

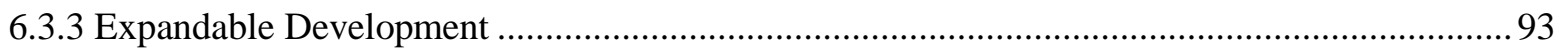

6.4 Sensing, Storage, and Transmission Summary and Conclusions ............................................. 97

Chapter 7 System-Level Design Summary and Analysis ............................................................... 98

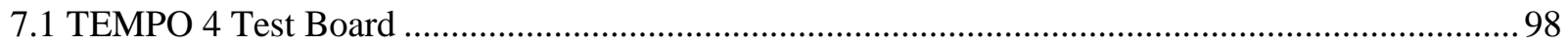

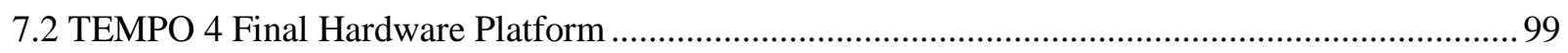

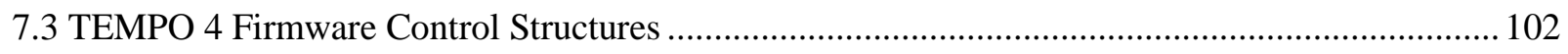

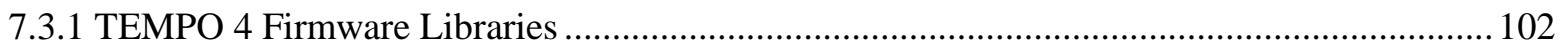

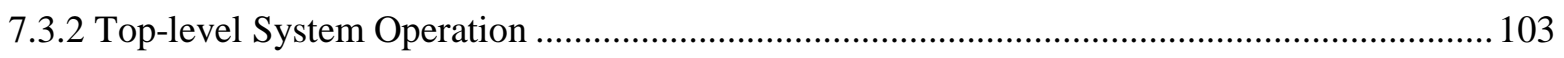

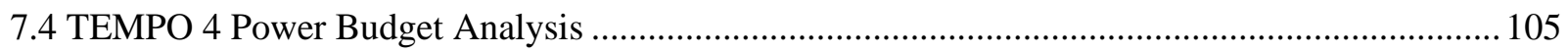

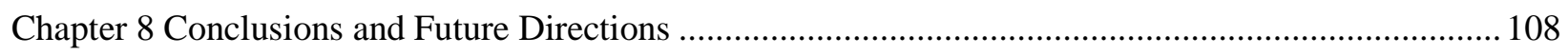

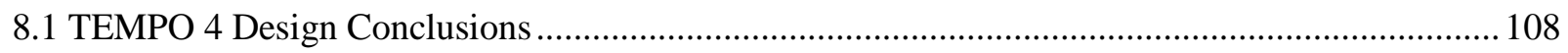

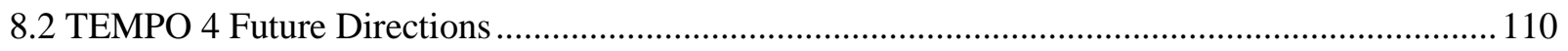

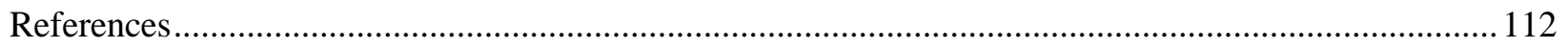

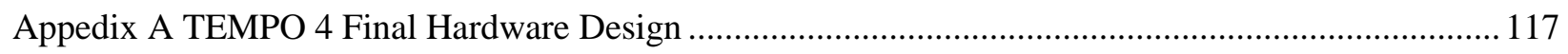

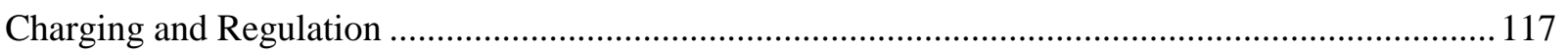

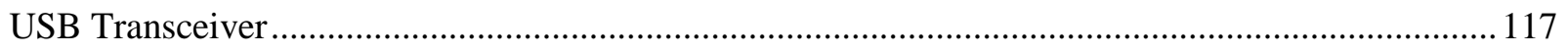

IMU

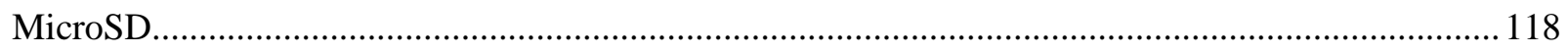

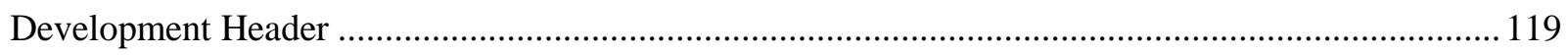

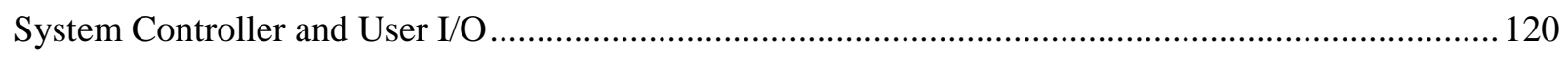

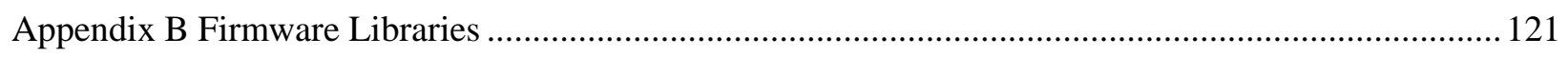

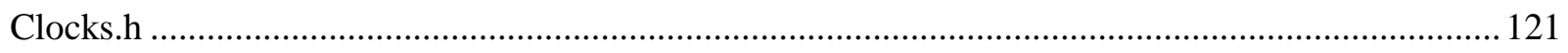

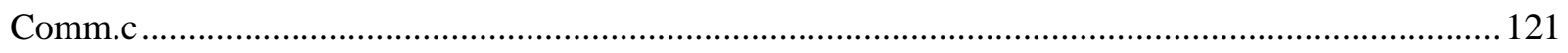

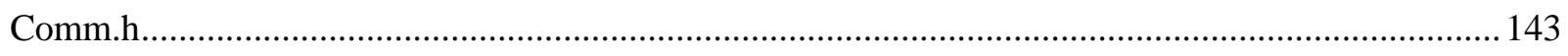

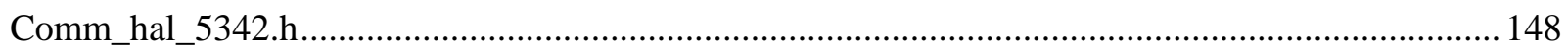


Command.c

Command.h..

Filesystem.c 156

Filesystem.h 163

Flash.c 166

Flash.h. 169

Ftdi.c 170

Ftdi.h 171

Hal.h. 173

Infoflash.c 175

Infoflash.h . 179

Interrupts.c . 181

MMC.c 183

MMC.h 189

MPU.c 191

MPU.h. 200

RTC.c 207

RTC.h.. 208

Timing.c 209

Timing.h.

Util.c 212

Util.h 213 


\section{Table of Figures}

Figure 1: Academic BSN Development Flow .............................................................................. 1

Figure 2: INERTIA Team BSN Research and Design Approach...................................................... 2

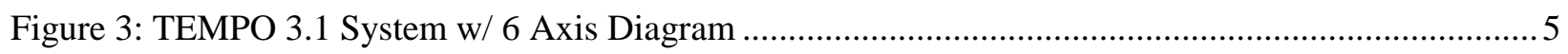

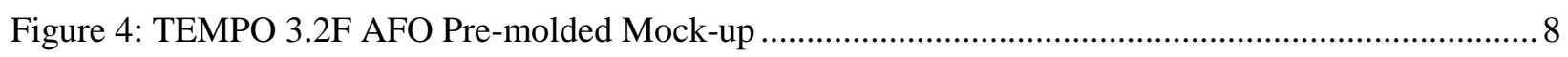

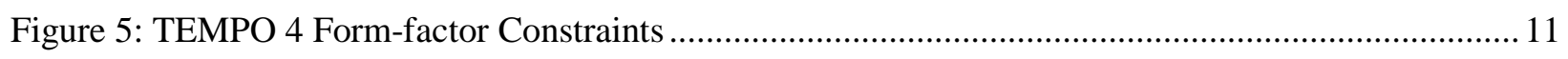

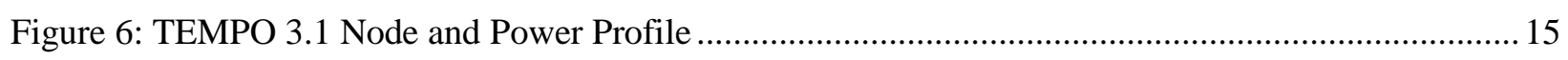

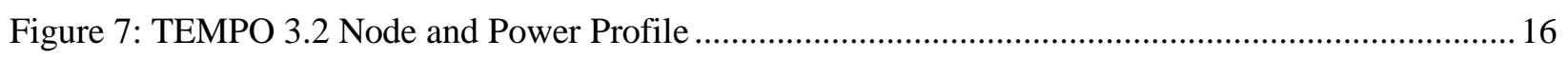

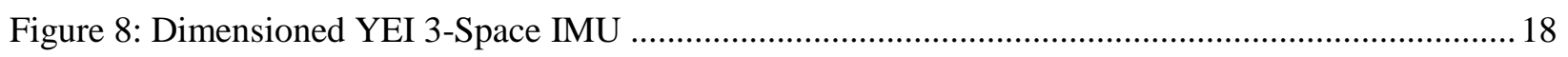

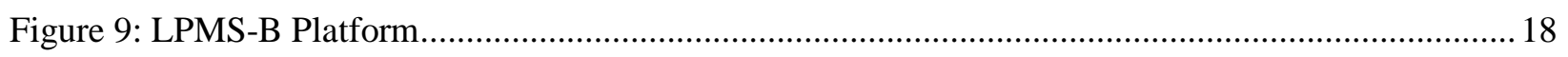

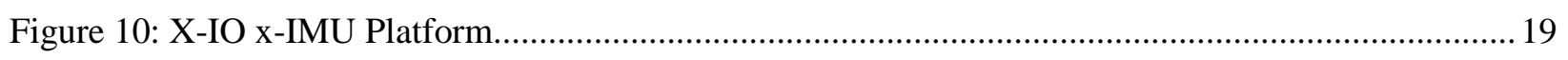

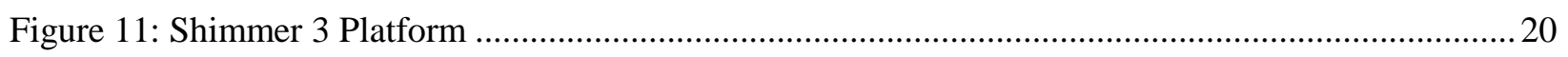

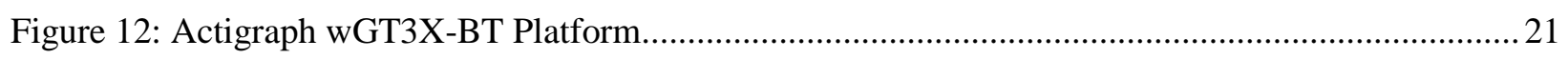

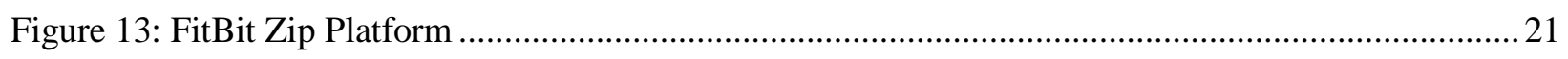

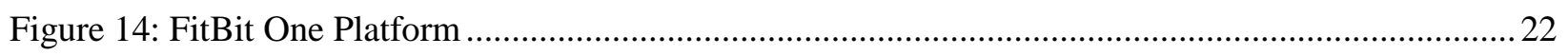

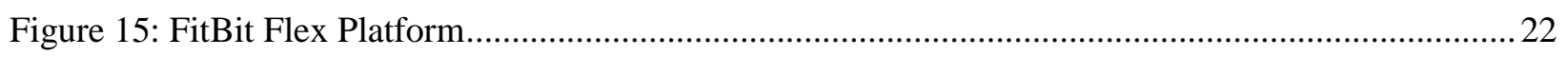

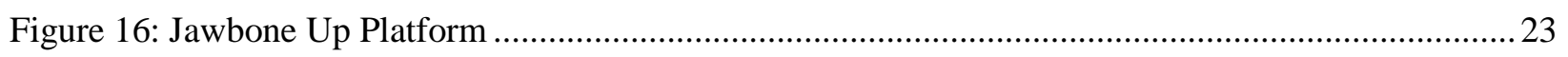

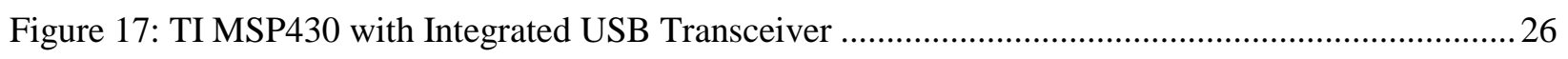

Figure 18: Block Diagram of FT232 Low-speed USB Transceiver IC …............................................2

Figure 19: FT121 SPI-driven ASIC Evaluation Module ..................................................................2

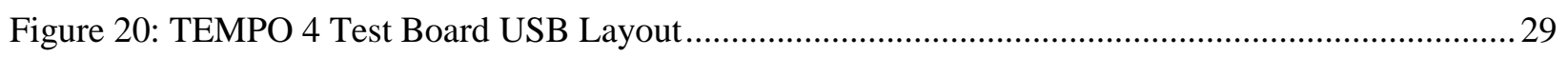

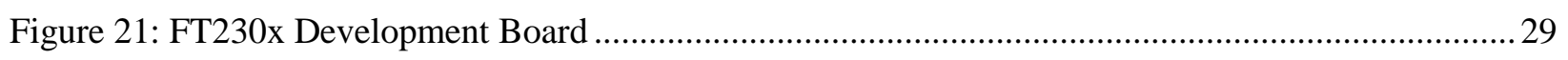

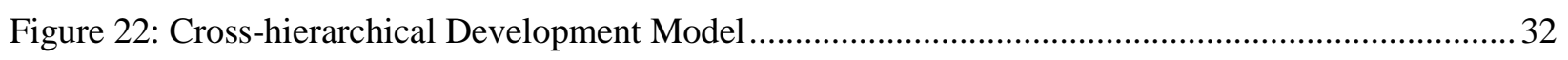

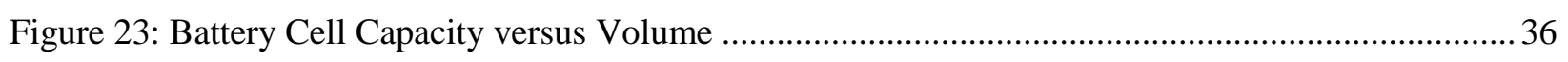

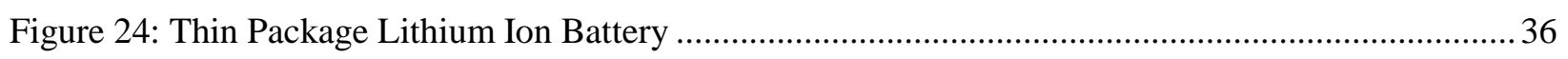




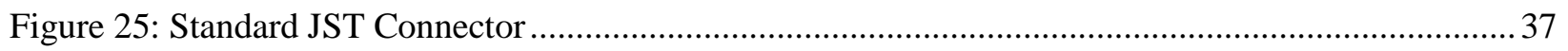

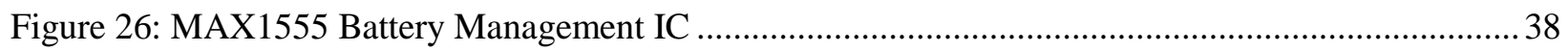

Figure 27: MAX1555 Low-Passive Count Charger Circuit ................................................................ 38

Figure 28: Low-Profile Reverse Voltage Protection Circuit................................................................ 39

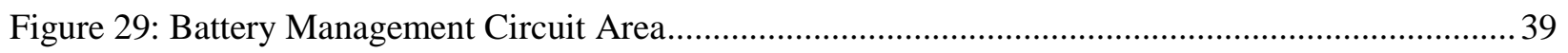

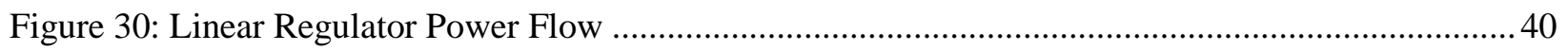

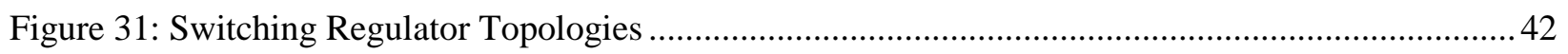

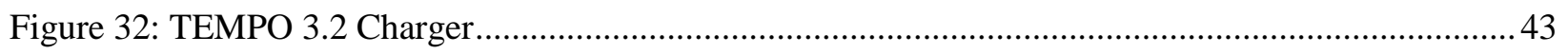

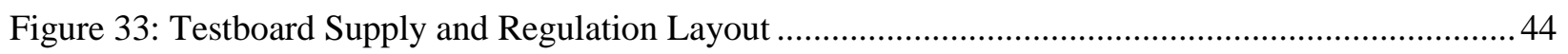

Figure 34: TEMPO 4 Battery Supply and Regulation Circuitry ......................................................... 44

Figure 35: Cypress Programmable SoC System Topology …......................................................... 47

Figure 36: SI Lab Split I/O Crossbar Switch Design ....................................................................... 48

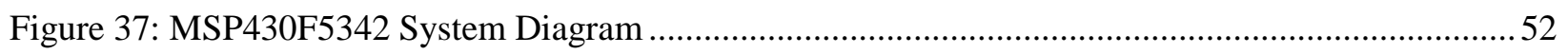

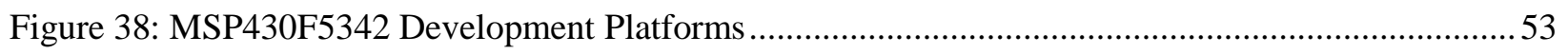

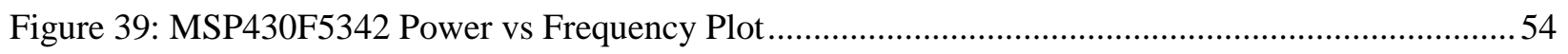

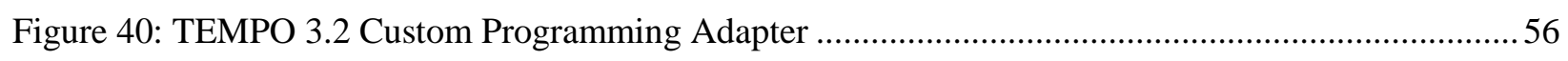

Figure 41: TEMPO 3.1 and 3.2 High-density Programming Connectors..............................................56

Figure 42: Texas Instruments Spy Bi-Wire Operational Concept ….................................................57

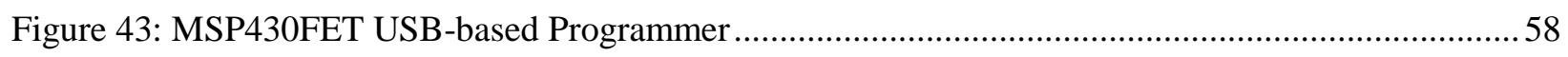

Figure 44: MSP430 Launchpad Platform as a SBW Programmer......................................................58

Figure 45: Launchpad Debugger Connected to the TEMPO 4 Test Board ............................................59

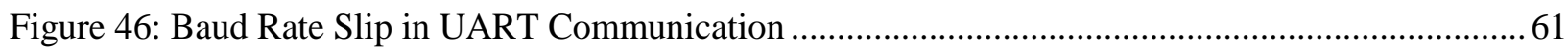

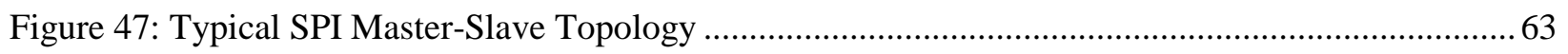

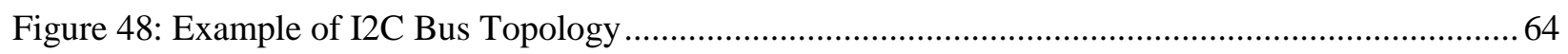

Figure 49: TEMPO 4 Communications Library Operational Hierarchy...............................................67

Figure 51: Final TEMPO 4 Design Demonstrating Breadboard Interfacing .........................................6 68 
Figure 50: Dimensioned Drawing of Maximum Allowable TEMPO 4 Pin Header .68

Figure 52: TEMPO 4 16-Pin Development Header Pin-out .69

Figure 53: Final TEMPO 4 USB Transceiver Schematic and Layout..... 72

Figure 54: TEMPO 2 Platform with Two User Push-buttons............................................................... 72

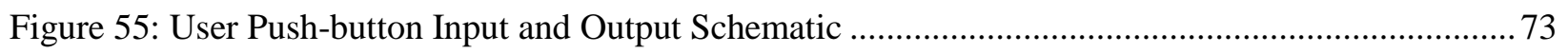

Figure 56: TEMPO 3.2 Board Area Devoted to IMU Solution .......................................................... 74

Figure 57: 13x13mm iNEMO 9DoF IMU Module from ST Microelectronics .......................................75

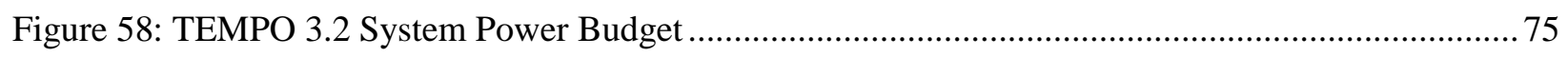

Figure 59: MPU9250 9 DoF Motion Capture Platform with I2C or SPI Interfacing ............................... 77

Figure 60: Pin-compatible MPU6xxx and 9150 IMUs in 4x4mm QFN Package ..................................77

Figure 61: Area Overhad Affiliated with MPU6/9xxx Motion Sensing ............................................... 78

Figure 62: Custom MPU6000 Breakout and SparkFun MPU6050 Relative ........................................... 78

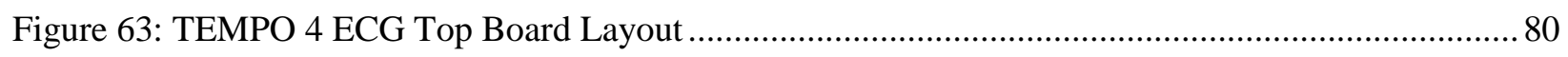

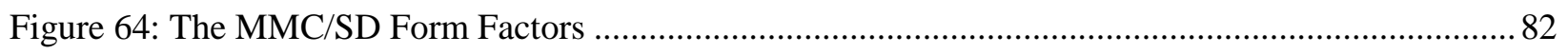

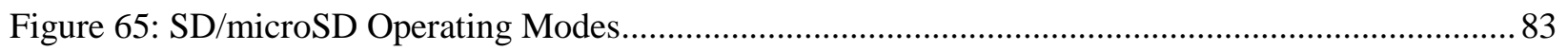

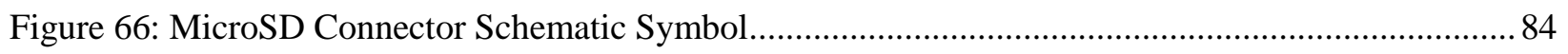

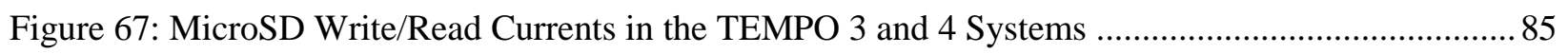

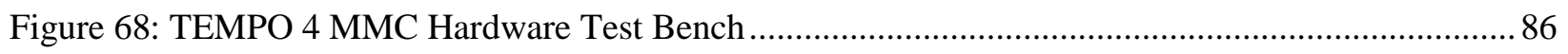

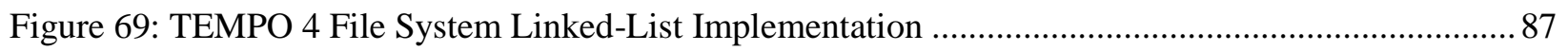

Figure 70: Power Consumtion versus Data Rate of Several Radio Protocols ......................................... 89

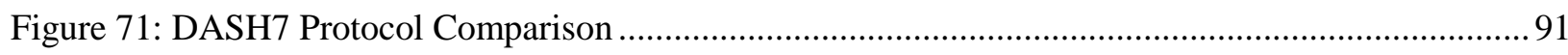

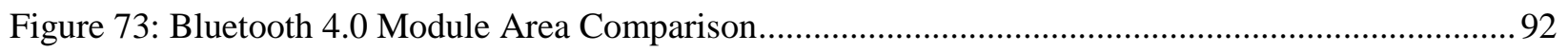

Figure 72: RN-41 Bluetooth 3 Radio Module used in TEMPO 3 Systems .......................................... 92

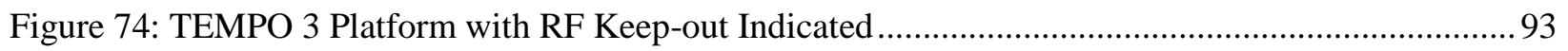

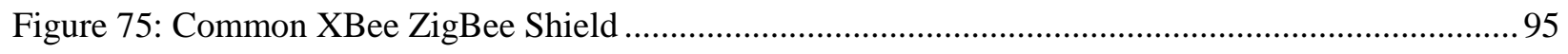

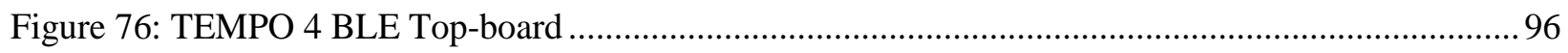




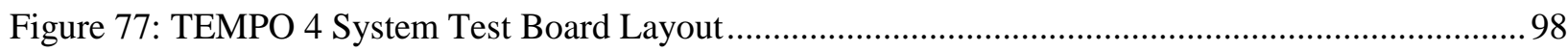

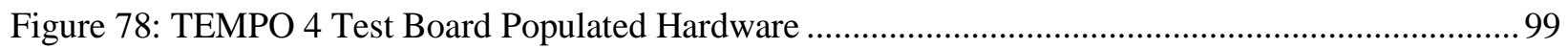

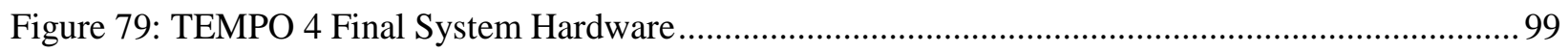

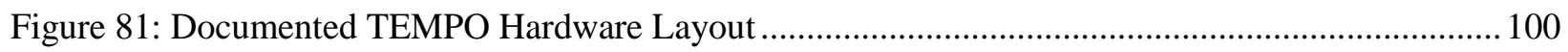

Figure 80: TEMPO 4 Final Board and Previous TEMPO 3.2 System Main-board................................ 100

Figure 82: Approximate Power Budget for TEMPO 4 in the 3 and 6 DoF Use Cases........................... 105

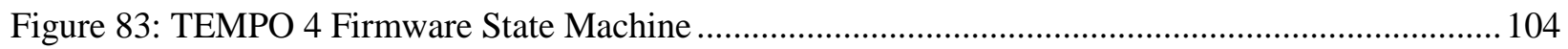

Figure 84: Predicted TEMPO 4 System Power Budget at Various Operating Frequencies ..................... 106

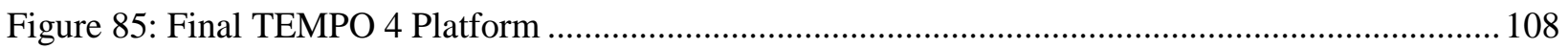




\section{Table of Tables}

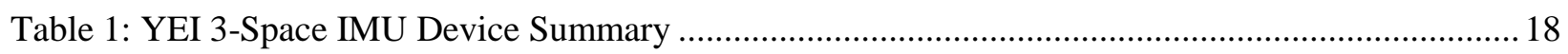

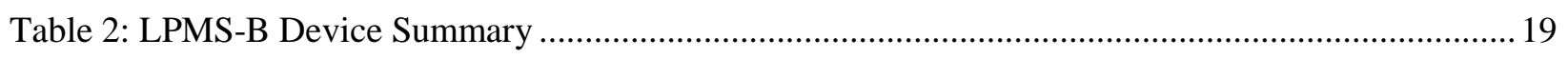

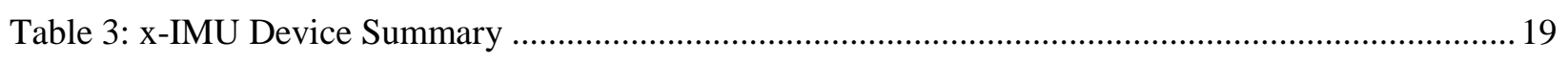

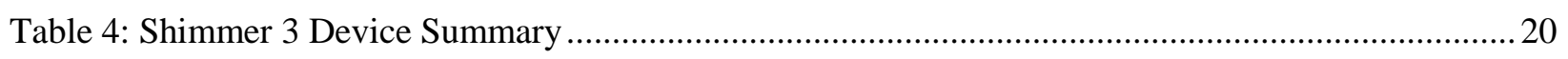

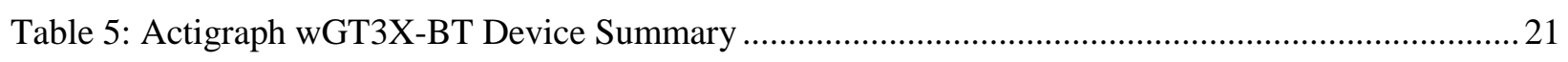

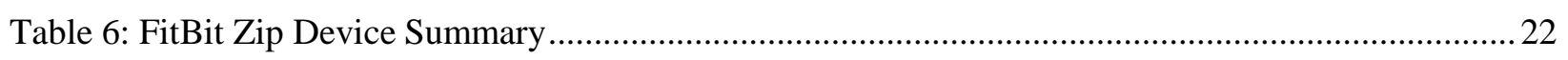

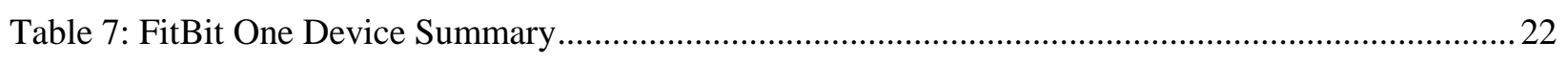

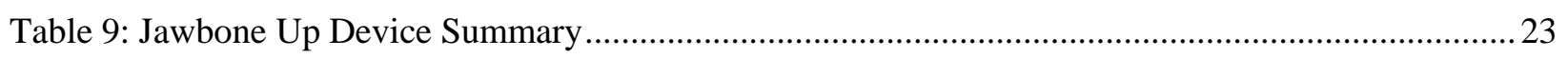

Table 10: Surveyed Commercial Alternative Market Comparison......................................................2 24

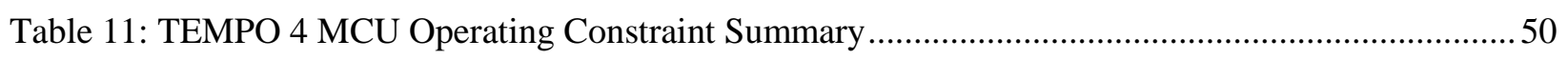

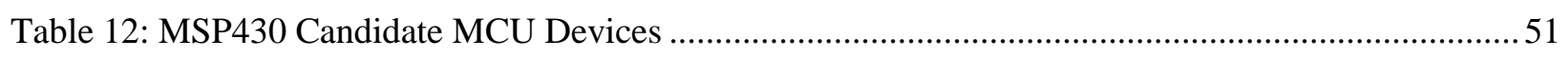

Table 13: MSP430F5342 Power Management Mode and Core Operating Condition Definitions.............54

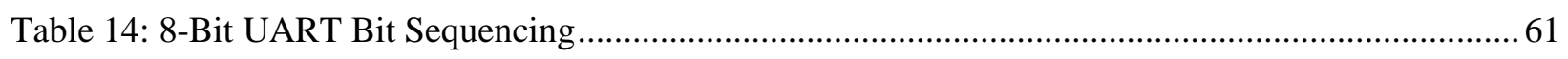

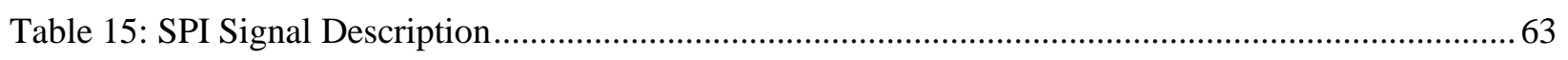

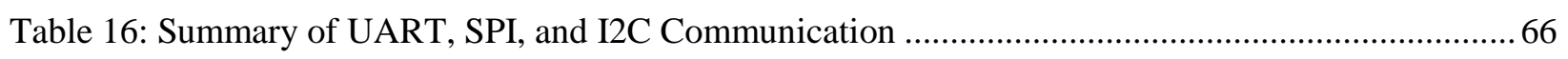

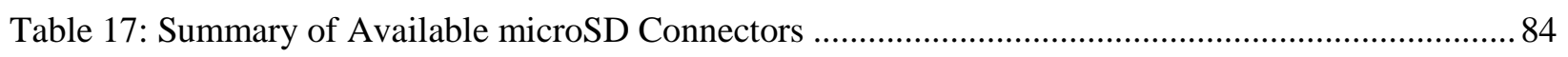

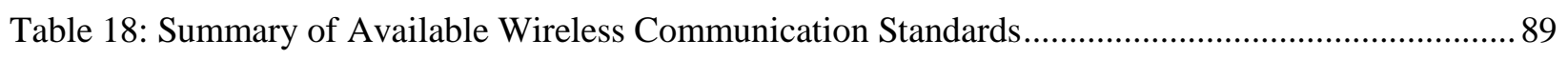

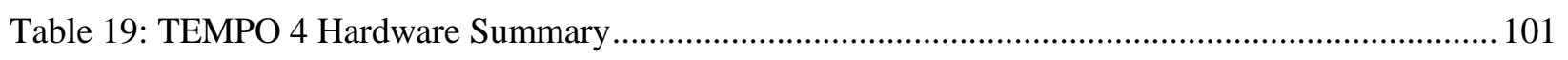




\section{Chapter 1}

\section{Introduction and Motivation}

Developing deployed sensing platforms in the academic context presents some challenges which are generally applicable to system designers and others which are unique to this design space. One of the most interesting challenges addressed by this work is that of the outward tapering of applications and deployments in the research context, as demonstrated in Figure 1. As a result of this taper, system designers need to consider a wide set of possible next-generation deployments while continuing to support the legacy needs of some or all of the previous deployments. In the case of the TEMPO platform this means maintaining, and possibly improving, the inertial motion capture capabilities of the device, while simultaneously extending the hardware into new deployments where inertial motion may not be the primary sensing modality.

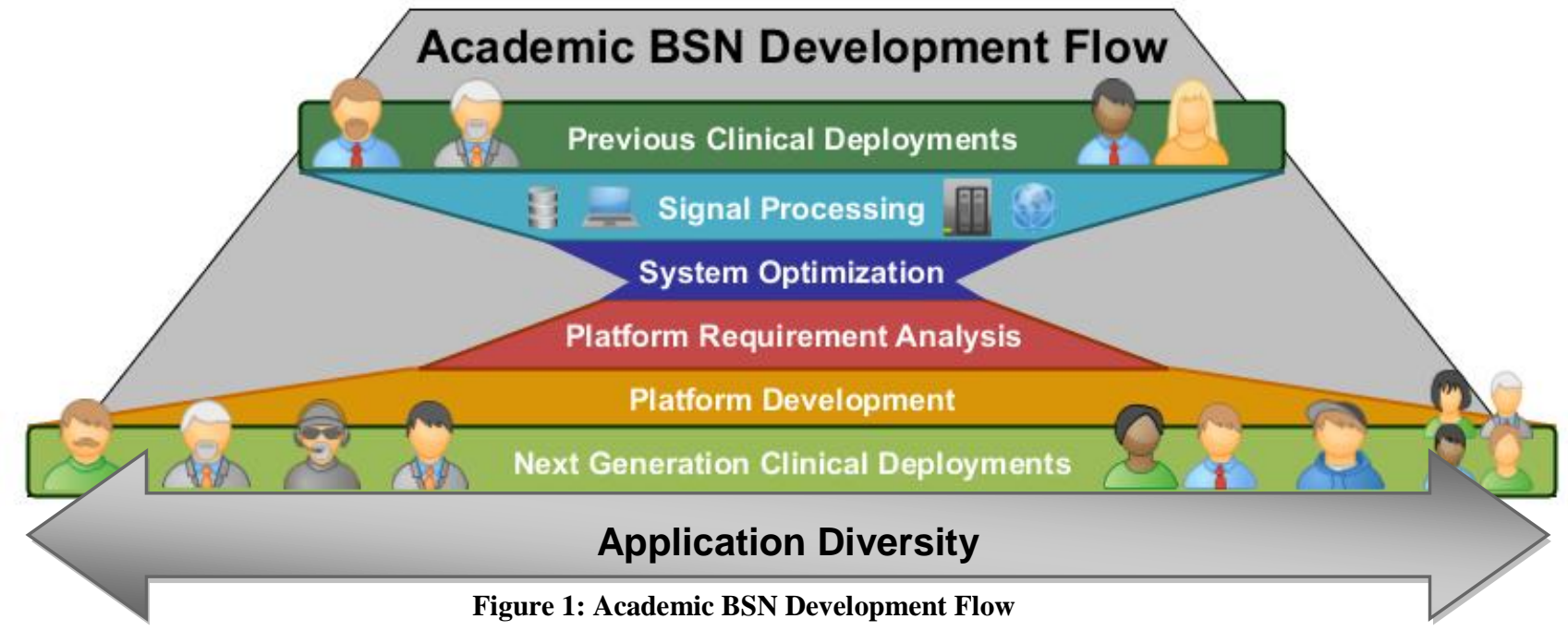

The TEMPO 4 platform features multiple interfaces for acquisition, including analog sampling and digital communications capabilities. In addition it supports on-board 6 Degree of Freedom (DoF) motion sensing, battery management, voltage regulation, and flash-based, microSD data storage for ultra-low energy operation. By leveraging pre-built and rigorously tested code libraries for node development and control this work seeks to both increase ease of development and improve robustness of operation for long-term, remote deployments in energy constrained environments. 
The design and deployment of body sensor nodes in real-world patient-physician environments has long been one of the unique draws of the INERTIA team at the University of Virginia. By engaging technical and professional collaborators, both in the applications and development spaces, the group has created a cyclic development model with the intent of finding new opportunities for cross-hierarchical design optimization and improvement of the end-user experience for doctors and patients. This work seeks to both motivate and demonstrate the next iteration of this process in examining important conclusions drawn from the development, production, and deployment of the TEMPO 3.1 and 3.2 platforms and describing the development of the next generation of TEMPO devices.

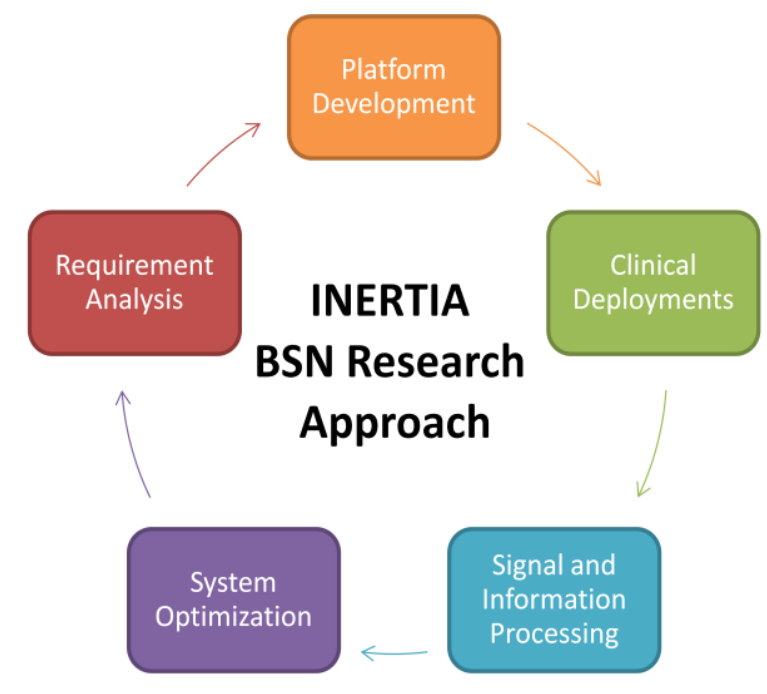

Figure 2: INERTIA Team BSN Research and Design Approach

The goal of this research approach, when viewed as a design cycle, is to both inform system designers of the needs of clinicians and signal processing experts working actively on the platform and also to make these collaborators more aware of the low-level capabilities and limitations of both the platforms they are currently using, and what may come in the future. By distributing lower level knowledge of the system's operation to technical collaborators this approach seeks to enable improved application-driven platform development.

The content of this works focuses primarily on the system optimization, requirement analysis, and platform development portions of the INERTIA body sensor network (BSN) research approach, and is organized around the activities that take place in each stage of this hybrid research/design flow. In the system optimization phase, information collected from physicians, technical collaborators, and signal processing experts is synthesized into a set of targeted deliverables for the next generation of the platform. During requirement analysis, the primary challenges proposed by the conclusions made during 
the system optimization phase are analyzed. Based on these challenges subsystems are created and specifications are created. Finally, during the platform development stage parts are selected, evaluated, and tested based on the previously developed subsystem specifications, before being synthesized into a full, working, top-level system.

\subsection{Understanding the Design Space}

The first challenge to address in developing a platform for the ULP body-worn context is the determination of critical features and metrics for the design space. This section will introduce some of the key challenges and opportunities facing COTS developers working in the body-worn context today. It also includes some background information along with a brief introduction to the field of on-body inertial motion capture.

\subsubsection{Significant Metrics}

One of the primary considerations for any designer trying to specify constraints for a platform is that of significant metrics for consideration. There are myriad traditional metrics considered as standards in the field of embedded design; however, the applicability of these standard metrics to the academic research design space may vary. For example, some metrics, such as unit-cos-at-volume or the ability to source large amounts of components, are not as critical concerns in the academic context, while others, such as time-to-design and measurement accuracy, can be even more critical than their industry standard counterparts.

Though arguments can be made for a number of valid figures of merit for evaluation of platforms, this work will focus on five targeted metrics determined both from previous experience and general market directions. These are as follows:

- Battery life: Battery capacity and system power considerations

- Form-factor: Size, weight, and shape in the on and off-body contexts

- Reliability: Predictable control and robust operation

- Ease of interfacing: Offering common, commercially compliant interfaces for communication

- Flexibility: Rapid expansion and prototyping on an academic platform

There are, of course, trade-offs that also exist within these targeted metrics. A few of these trade-offs will be discussed briefly in the remainder of this section. Part of the contribution of this work is the demonstration of several general techniques effective for helping to produce separability in some of these trade-offs, simplifying decisions for the system designer. In other cases, where such general separability 
is not possible, the complex trade-offs that occur near or at the hardware-firmware boundary are addressed using application-specific knowledge and prior experience in the design space.

One design trade-off implied by the set of metrics proposed above is that off battery capacity versus formfactor. Most system designers are aware that they can typically trade off increased size for increased capacity in most, if not all, battery chemistries. This work attempts to produce some amount of separability in various technologies by using the typically referred to energy-density metric and by providing the battery life metric in hours at a rated capacity, allowing for easy linear extrapolation of lifetime when using alternative capacity batteries of similar chemistry.

A second trade-off is that of flexibility versus reliability from a top-level system perspective. Often, reliable system operation in the presence of end-user integrated code requires heavy levels of program management and underlying interface code. However, the limited code-size and low clock-speeds of lowpower embedded microcontrollers (MCUs) often results in an inability to produce such complicated control structures. A significant contribution of this work is addressing this challenge in regard to multiperipheral hardware communication interfacing on a commercially available MCU.

A final, and possibly less intuitive, trade-off existing among the candidate metrics proposed above is that of ease of interfacing versus form-factor. The principle challenge here resides not only in the significant amount of board area that some of these physical interfaces can consume, but also in integrating widely accepted wireless interfaces, such as Bluetooth or Zigbee, into low form-factor designs. This issue will be discussed at length in the chapters to follow, as the radio-frequency (RF) communication challenge may be amongst the greatest facing wireless on-body sensor node developers.

\subsubsection{Inertial Motion Capture}

The TEMPO platform, which will be described in greater detail in the following chapters, exists primarily for the purpose of the wireless capture of three-axis acceleration and rotation vectors from the wearer's body motion. This type of signal acquisition will hereby be referred to as six degree-of-freedom (DoF) motion capture, as it captures 6 , correlated, but independently measured axis for determination of motion in the global frame. An example of the orthogonal orientation of the 3 accelerometer axes and 3 gyroscopic planes can be seen in Figure 3. 

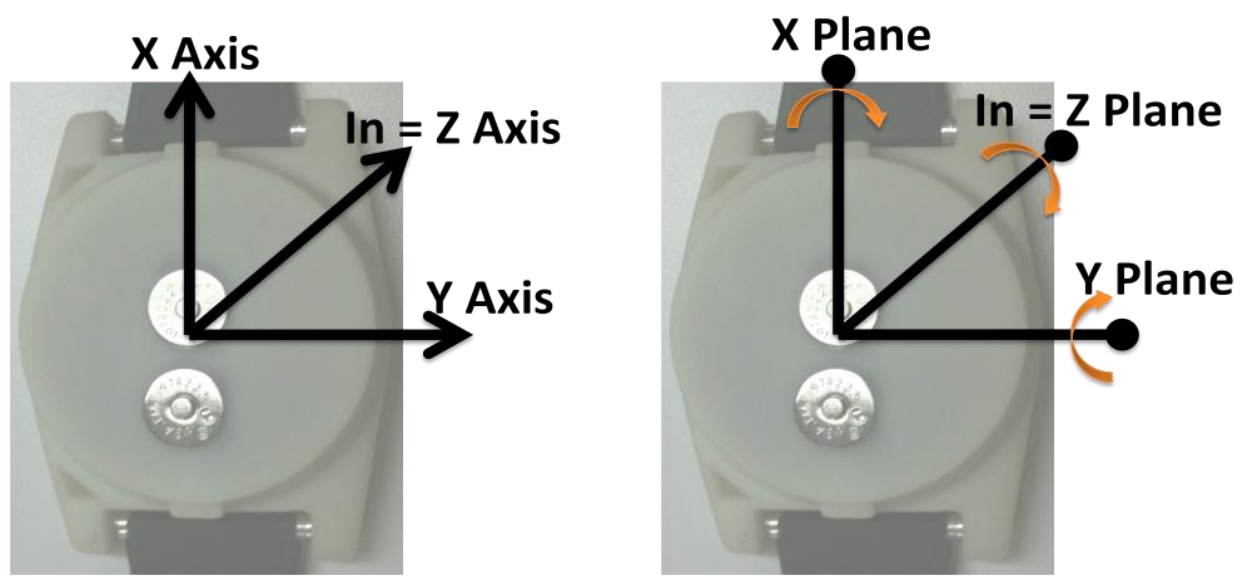

Figure 3: TEMPO 3.1 System w/ 6 Axis Diagram [1]

The uses of this inertial motion data are diverse. A number of medical studies in the areas of fall detection [11][12], gait analysis [13][14][15], and parkinsonian tremor [16][17][18] have all showed significant promise, and the field of possible applications is still growing. Meanwhile, a simultaneous interest from "quantified selfers" or that portion of the consumer market which is interested in tracking of personal metrics for wellness or fitness, has promoted a commercial explosion in the wearables space. This work attempts to deal primarily with the challenges faced by embedded developers interested in designing inertial sensing platforms for this rapidly expanding context, enabling key academic deployments while maintaining a competitive edge when compared to more tightly-integrated commercial products.

The challenges facing designers in the wireless motion sensing space are diverse. In the field of sensing, more recent interest in body-worn activity monitors has begun to drive demand for lower power Inertial Measurement Units (IMUs). These IMUs use Micro Electro-Mechanical Systems (MEMS) to produce output voltages proportional to the acceleration or rotation seen by a single point in the MEMS element. Traditionally IMUs were high-power devices, used in aeronautics and slowly introduced into the automotive market, but as demand for low-power IMUs designed for lower-power applications has grown, so has the corresponding market share.

Most traditional IMUs integrated one, or possibly two axes of accelerometer or gyro-based monitoring integrated in custom physical foot-prints to accommodate the specialized electrical and mechanical consideration of the device. These earliest accelerometers and gyros used spring-mass systems, rather than the more modern MEMS-based technology, which had to be precisely tuned and calibrated for proper operation. This resulted in relatively high cost and low availability of these devices to most COTS developers. As the automotive industry began to more widely adopt accelerometer into vehicles, primarily for collision monitoring, the level of integration of the accelerometer grew quickly. As the MEMS field 
developed rapidly to accommodate this new desire for electrical orientation sensing, gyros also benefited from advancements such as reduced feature sizes and increased level of silicon integration, allowing for standard packaging and single-chip multiple axis sensing.

The previous TEMPO platforms have all implemented MCU-side analog-to-digital conversion [1]. This was both because state-of-the-art IMUs were all primarily offering analog interfaces at the time, and that this scheme allowed for precise control and timing for the MCU-side Analog-to-Digital Converter (ADC). As a result of previously mentioned increased level of integration, today, many low-power IMUs take advantage of on-chip signal conditioning and integrated ADCs to provide a simpler all-digital interface to the user. Along with this simplified interface, also comes the power savings of not buffering and processing analog signals on chip. Last, but not least, the recent boom in MEMS miniaturization and lowpin count of these all-digital interfaces mean smaller package size and low off-chip passive counts for the new generation of all-digitally interfaced IMUs.

Efforts affiliated with the capture of human motion data have arrived at varied Nyquist criteria and tolerable phase offset in system sampling [2]. While most prominent work agrees most spectral content of interest lies between 0 and $12 \mathrm{~Hz}$, sampling rates as high as $1 \mathrm{kHz}$ are commonly used to digitize human motion data. These higher sampling rates are often exploited by complex, estimation theory-based signal approximation techniques, such as Kalman filtering, which can take advantage of the information benefits obtained from over-sampling of the mostly sparse spectrum of interest. Based on a number of previous high-fidelity human motion capture deployments, along with extensive involvement in the early development of signal processing techniques for the body-motion context, the INERTIA team has arrived at $16-128 \mathrm{~Hz}$ as an acceptable range of sampling rates for extracting meaningful information for most human motion capture deployments.

A number of differing conclusions regarding digitization bit-depth along with the use of on-node compression or decimation to reduce output data rates have also been discussed in regard to on-node sensing. Within the INERTIA team's open firmware development model, much of this sort of control is accessible to the application coordinator prior to deployment. This means that nodes can be quickly modified to sample at various rates and perform relatively simple, user-defined data tasks.

\subsection{State of the Art Platforms}

A more in-depth review of state-of-the art platforms for on-body motion capture is conducted in the following chapter of this work; however, to demonstrate the motivation for this design and unique features of the TEMPO platform, a brief discussion of state-of-the-art IMU systems is provided below. 
Generally speaking there are two categories of commercially available IMUs on the market today. As a result of recent popularity in the health and fitness markets $3 \mathrm{DoF}$, accelerometer-only, fixed functionality platforms have become much more common, predominantly as pedometers. This has lead to significant improvements in battery life through high levels of integration and iterative improvemnt. For reference, today's state-of-the-art pedometer-based IMUs can run for up to 6 months on a single, non-rechargeable coin cell battery [3]. The previous TEMPO platforms also fell into this category of IMUs and for this reason, have in some ways failed to stay competitive in the face widely available, low cost commercial alternatives.

The second class of IMU platforms considered for this work is referred to as "expandable" devices. These are primarily fully or semi-open development platforms with hardware and/or software interfaces are provided for configuration, programming, or customization. Typically these devices are produced by academics or commercial companies with active interest in engaging with the academic research community. As of today, relatively few platforms in the body-worn context have adapted this flexible style of development, with a few notable exceptions [4] [5]. However, in the more mainstream development community, the flexible development model has become commonly accepted, with a large pool of open hardware and firmware developers working on top of platforms such as Arduino, RasperryPi, and Maple.

In order to best suit the needs of on-going developments and future correlative studies implementing additional, or entirely new sensing modalities this work focuses on the second category of IMU platforms. In addition to fitting the needs of on-going TEMPO deployments, this focus on expandability and userinterfacing means that the TEMPO 4 platform represents a significant step forward in the form-factor and power constraints not only relative to previous TEMPO nodes, but also the state-of-the-art in available commercial products.

\subsection{Motivation and Device Specifications}

This work is motivated by the need for a low-power, wearable and expandable IMU with open hardware and firmware for the research community. This work addresses challenges similar to those of the leading low-power 6 and 9 DoF platforms, but adds an increased focus on availability of standard digital interfaces and firmware libraries designed for reliable, low-overhead operation during the rapid prototyping and proof-of-concept phases of design.

Several key pieces of feedback from the medical deployment and signal processing phases of the previous BSN design flow seen in Figure 2 are fed forward into this design. Namely, several cases in which the 
TEMPO 3 system failed to meet the evolving needs of medical collaborators, and increased demand for capabilities for on-node signal processing from technical collaborators have promoted a new hardware/firmware iteration of the TEMPO system. The system optimization portion of this work consists primarily of the evaluation of the previous TEMPO 3 platform in the context of modern deployment demands, shifting COTS market direction, and increasing relevance of hardware-firmware co-design concepts.

\subsubsection{Deployment Challenges and the TEMPO 3.2 AFO}

Recent deployments of the TEMPO 3.2 system have demonstrated both the value of robust, reliable operation during long untethered deployments and the added value of being able to rapidly prototype a new sensing platform using an existing IMU.

There was relatively little negative feedback from collaborators regarding the operation of the TEMPO 3.1 and 3.2 Bluetooth-based systems, as typically a technical collaborator was in the room monitoring the data collection. There were however, some limited complaints about the quality of calibration and inability to synchronize two nodes sampling rates to one another. In addition the relatively short battery life of these nodes prohibited more longitudinal deployments. Though capable of performing much longer data sessions, and coupled with a rather user-friendly offload interface, the TEMPO 3.2 flash-based node received a far greater amount of negative feedback. Due to the complex nature of the firmware and operating model, collaborators often accidentally left the device on and sampling for hours, draining the battery and in some cases producing critical errors that locked the node from operation.

In one case, two TEMPO nodes were paired together for the purpose of synchronizing system sampling rates as part of an effort to determine the ankle-angle of an ankle-foot orthotic (AFO) device. Though the TEMPO 3.2 hardware and firmware were fortunately able to be modified to source and sink system clocks through exposed pins, this was not intended functionality of the device. However, the

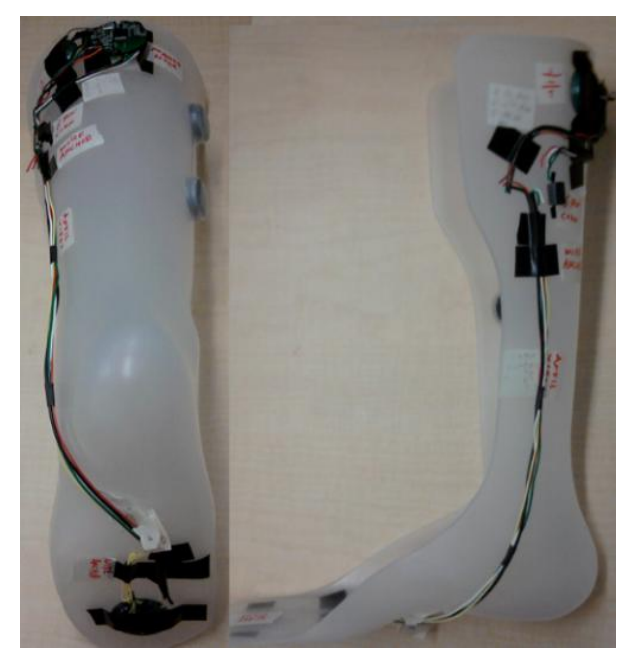

Figure 4: TEMPO 3.2F AFO Pre-molded Mock-up precise nature of monitoring small changes in ankle-angle via body worn 6 DoF sensing proved incredibly difficult. Through careful synchronization of system sampling rates, and a collaborator's development of more complex, non-linear descent method-based software calibration schemes, this device was proven able to accurately recover the information of interest. The resulting AFO system mock-up is shown in Figure 4. 
The system shown above functions using two TEMPO 3.2 flash-based nodes, one designated master and one slave. The master uses its on-node $32.768 \mathrm{kHz}$ crystal oscillator to source a stable digital clock signal to the slave node, which receives a conditioned version of this clock signal for sourcing its own lowfrequency external oscillator inputs. A unified charging and communication port was created by connecting the charge inputs of the two nodes, and establishing a keyed connection to maintain polarity of two separate RS-485 data connections. The hardware produced, though a bit shaky to begin with, ended up performing reasonably well in a custom-molded AFO.

Unfortunately, the final goal of this project is the molding of custom children's AFO's, which are a great deal smaller than the adult counter-part shown above. For this reason, the size of the TEMPO 3.2 platform, with or without its battery, prohibited the use of TEMPO 3.2 in this device as a long-term solution. One proposed solution was the use of a smaller daughter-board, designed explicitly to be mounted on the bottom of the foot, sourcing its power and clock from the larger master node which could remain on the upper thigh. However, the TEMPO 3.2 platform supported no such easy-to-use interface, and would require major modification and possibly even a new layout all together, to achieve this formfactor.

The result was the demand for a platform capable of providing reliable power to and interfacing a lowprofile daughter board designed to be situated on the bottom of the foot of a children's AFO. The TEMPO 3.2 node offered no ability to implement such a daughter board. As a result, it was deemed that this final AFO product could not make use of the current TEMPO platform. The hardware design challenge presented by this AFO project was a significant part of the motivation for a new TEMPO system at the onset of this work.

\subsubsection{On-Node Signal Processing and Interface Considerations}

As the set of applications of the TEMPO 3 platform grow more diverse, so does the signal content of interest. Though in the past, many deployments required high-fidelity monitoring and burdensome levels of signal processing on the back-end, many newer deployments look increasingly to on-node processing efforts to increase battery life and decrease back-end data bloat and processing complexity. One practical application demonstrating the value of increased on-node signal processing was that of a data-driven power reduction technique proposed by a technical collaborator near the onset of this work.

Our collaborator noticed that more than $50 \%$ of the power being consumed in the TEMPO 3.2F power budget is that of the 3 axes of gyro sensing used by the node to precisely capture changes in angle. The collaborator then connected this information with the application knowledge that when an individual is 
sitting still, these high-fidelity gyro signals provide little-to-no information to the signal processing expert. This connection allowed him to develop a simple piece of code that calculated the standard deviation of the vector magnitude of the 3-axis accelerometer signal, and based on simple thresholding with hysteresis, decided whether or not to turn the gyros on or off. This type of sensor-integrated control presents significant power reduction opportunities for many high-fidelity motion capture platforms and demonstrates the value of developing low-level power control schemes with knowledge of high-level application constraints.

In addition to feedback on providing additional capability for on-node processing, the INERTIA team has also heard increased demand for the ability to run more burdensome libraries and Real-Time Operating Systems (RTOSs) on the TEMPO hardware. This promotes an interest in increased maximum system clock rates and large on-chip instruction memories for those who chose to pay for their development tools.

\subsubsection{Open Development Considerations}

The final motivating factor outside of the desire for a more powerful, expandable platform designed to compete with the best the market has to offer, is making the TEMPO 4 design an open and available resource for the embedded development community. By allowing individuals to work on top of the platform, building their own hardware and firmware extensions, this work seeks to maximize the set of applications it is capable of being deployed in. While simultaneously, by allowing individuals to modify and reproduce the core platform itself, this work hopes to reap the benefit of continued iterative improvement throughout its lifetime.

\subsubsection{TEMPO 4 System Specifications}

Rather than fully specify all of the operating characteristics of the final TEMPO 4 platform here, instead the specifications for the targeted metrics, introduced at the start of this chapter, will be established and some strong top-level system constraints put in place for the remainder of the design process. Below, a brief specification of each of the desired metrics is provided.

\section{Form-Factor}

The TEMPO 4 platform form-factor is one of the strong constraints that will be put in place early in the design process. Based on a desire to be reverse compatible with older, custom printed casings it was decided that the TEMPO 4 node need be able to be inscribed within the existing footprint of the TEMPO 3 devices. In addition, rather than using the more difficult to manufacturer circular design implemented to mimic a wristwatch in the TEMPO 3 platforms, this device targets a simpler, rectangular geometry for 
low-cost panelized mass production. This rectangular board shape requirement, together with the inscription-constraint introduced earlier, imply that if the TEMPO 4 platform is to maximize the usable area within the previous node's footprint, it will need to be a square of maximum allowable side-length.

Thus, as one of the first considerations of this work, it was decided that the TEMPO 4 platform would be designed to fit within a $1 \mathrm{x} 1$ inch footprint. Thus, throughout this work, all circuit area computations will be carried out relative to a 1 square inch board size. It is worth noting at this point that a significant amount of effort is not put into characterizing the system depth dimension, since the height of the end-user

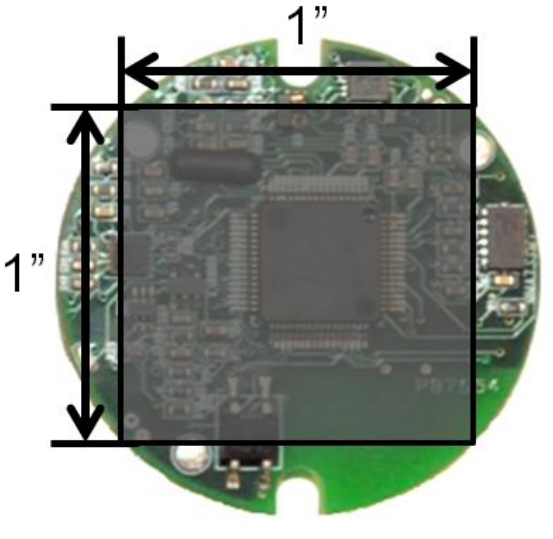

Figure 5: TEMPO 4 Form-factor Constraints Based on Device Footprint Inscription platform may vary with choice of power source and add-on modules.

\section{Lifetime}

It is difficult to come to general conclusions regarding lifetime for flexibly deployed sensor systems. In the use-case of 6 DoF inertial motion sensing, the TEMPO 3.2, flash-based platform was able to obtain 10-12 hours of continuous monitoring, and in the accelerometer-only case it was able to run for up to 30 hours. At the very least this work seeks to improve upon the lifetime offered up by previous TEMPO systems by a factor of 2, enabling one day of continuous 6 DoF data monitoring, though it will be shown later in this work that this bound can, with appropriate battery selection, be significantly out-performed.

\section{Interfacing}

The TEMPO 4 device needs to be able to communicate directly with a host computer or smart phone without use of custom hardware for interfacing. This constraint is derived primarily from the added design challenge of creating this custom hardware and the barriers to open development such interfaces can create. This work considers a number of standard interfaces for development, but as a bare minimum it is required at least one commonly available, commercially supported interface is provided on-board.

\section{Reliability}

While this section will propose no formal considerations for reliability, it is of course required that the TEMPO 4 system be able to capture and record 6 DoF human motion data accurately to a user, in real time and/or after a deployment has completed. It is worth noting that this metric is titled "reliability" rather than "robustness" as it is intended to be considered from a top-level system functionality 
perspective. Since the node hardware is offered up without packaging or a software back-end an argument for platform robustness will not be made. Instead, reliable low-level library operation in the presence of a variety of top-level control strategies is evaluated in the context of an interrupt-rich programming environment. Further discussion of considerations for reliable and robust system operation is included in the coming chapters of this document.

\section{Flexibility}

In addition to providing on-board 6 DoF motion sensing, the TEMPO 4 node is also be required to support a flexible development and/or programming interface. This interface is established to enable future developers to easily access a wide variety of sensors, and will be required to be able to implement at least one set of regulated output connections, several pins for common digital serial protocols, along with analog data capture, digital I/O, and possibly other user-defined functionality.

With these goals and specifications established it is now possible to more concisely describe the contributions of this work in the context of both the previous TEMPO platforms and the state-of-the-art in commercial platforms.

\subsection{Contributions}

There are three primary areas of contribution of this work. The first is the development of a low-profile, wearable, open hardware platform for expandable, human motion capture referred to as TEMPO 4 . The second is the development of reliable and rigorously tested firmware libraries for serial communication, timing/clock control, MMC and USB interfacing, user I/O, and event-driven system operation to run ontop of this hardware. The final contribution of this work is the identification of general trends in today's ULP body-worn design space and demonstration the importance of system co-design concepts in achieving significant power and area reductions without compromising flexible, robust operating principles.

The hardware contribution of this work is summarized in chapters 4,5, and 6 of this work, along with two co-design case studies proposed in chapter 3. It includes the development of a single board, 6 or 9 DoF IMU platform with USB interfacing, battery charging and regulation, MicroSD data storage, 2 push buttons, and 2 LEDs for user interfacing. Most importantly, this work tackles the challenge of providing additional user I/O by means of an open 16-pin development header, designed for rapid platform expandability. 
The firmware libraries created for the TEMPO 4 platform are, with minimal porting, capable of running on most, if not all recent MSP430 devices from Texas Instruments. These libraries asynchronously manage system communication and sampling and provide useful tools for setting up base-level peripherals such as the on-chip frequency lock-loop (FLL) and real-time clock (RTC). Contributions related to firmware are also discussed in chapters 4,5 , and 6 , with an affiliated co-design case study presented in chapter 3 .

The general contribution of this work is identification of trends and challenges affiliated with body-worn sensor system design in the modern context. Conclusions drawn from experience spanning the duration of this work and beyond, are provided throughout the document. Chapter 2 introduces the previous TEMPO platforms and other commercial state-of-the-art competitors. Chapter 3 defines the concept of co-design in the context of general operating principles and introduces two affiliated case studies.As previously alluded to, Chapters 4-6 each demonstrate more specific, subsystem related claims. Though this work defines these claims as "general" it is of course acknowledged that many of the conclusions arrived at throughout this work are feature of the technology of the time. Having said that, this work does attempt to demonstrate the value of considering trade-offs that exist at and beyond the hardware-firmware boundary. 


\section{Chapter 2}

\section{Survey of Prior Art}

This chapter is intended to provide somebackground on the previous TEMPO systems, along with commercial alternatives. Together with the previous chapter's section on motivation for this work, it is intended to serve as the justification for a need for a new revision of the TEMPO platform, along with the special considerations that will be given to digital interfaces and flexible platform development.

\subsection{INERTIA TEMPO 3.1}

The TEMPO 3.1 system represented a major step forward in wearable, single-board platform integration for wireless body sensor nodes. The node features 3 axis accelerometer and gyroscopic monitoring, hereby referred to as $6 \mathrm{DoF}$ inertial motion sensing, along with on-board regulation, battery management, a Bluetooth radio module, and an MSP430 mixed-signal processor from Texas Instruments for control and processing. The TEMPO 3.1 node made use of a separate charger platform for providing the $6.3 \mathrm{~V}$ input necessary for charging the lithium polymer battery [1].

The primary advantage of the 3.1 system, over the previous state-of-the-art, was the integration of gyros for high-fidelity motion capture applications. Though this was expected to result in increased power consumption, and corresponding decreased lifetime, these losses were tolerated to improve the overall information provided by the system. This gyro integration, coupled with an easy to use Bluetooth 3 interface and relatively simple, tethered device operating model made the 3.1 node an attractive candidate for a number of emerging studies, where rapid, in-the-field data collection proved invaluable.

As a result of its popularity, about 50 of these nodes were produced and deployed as part of a number of clinical studies over several years. Applications included fall detection [6], gait analysis [7]-[8], classification of Parkinsonian tremor [9], and agitation quantification [10]. The typical deployment methodology for these nodes included a technical collaborator, commonly a member of the INERTIA team, present in the room collecting data while a medical collaborator provided instruction to the patient and possibly relevant medical feedback both to the collaborator and the patient in real-time.

Expected downsides to the TEMPO 3.1 platform were its relatively high unit cost and difficulty of manufacturability. As this was a platform intended for academic use only, these challenges were not viewed as critical at design time. In addition and as previously mentioned, power losses to the gyros were 
anticipated, but tolerated in the name of higher output fidelity. A less expected result was the powerhungry nature of the Bluetooth radio, consuming near $50 \%$ of system power during data collection.
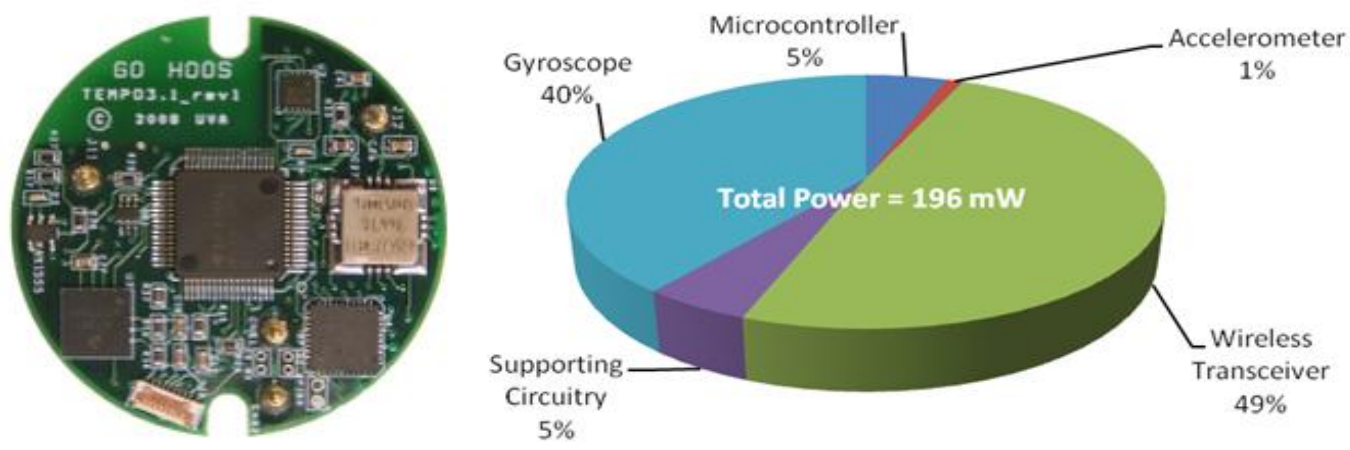

Figure 6: TEMPO 3.1 Node and Power Profile

With an overall average system power of about $196 \mathrm{~mW}$ the TEMPO 3.1 system could run for 4-6 hours on a single charge of a 300mAh LiPo coin-cell battery. This was deemed to be more than enough for the shorter in-clinic deployments the node was intended to suit. It is worth noting that some of the greatest challenges in TEMPO 3.1 deployments occurred in the few cases where nodes were passed off to, often technical, collaborators who were not intimately familiar with the platform. Though this was not an intended consequence of the development cycle, it was also not considered as an important metric for the deployment methodology in place at the time.

The take away points from the TEMPO 3.1 node design and deployment process are the value of a widely available interface and small-form factor, single board integration along with the challenges of wireless communication power and the energy-fidelity trade-offs proposed by the addition of gyroscopes to the IMU platform. Though the Bluetooth radio was in many ways the common-bridge to a number of aggregation platforms, it was also the Achilles heel of the system's power consumption, and provided integration challenges when it came to component cost and physical layout. Meanwhile signal processing efforts were vastly improved by the integration of rotational measurement into the inertial frame, and these hardware contributions were considered invaluable. The TEMPO 3.1 node was primarily heralded as a "high-fidelity" motion capture platform in lieu of its increased rotational sensing modalities and programmable sampling rate.

\subsection{INERTIA TEMPO 3.2}

The TEMPO 3.2 node represented the next iteration of the TEMPO design process. This node still hosted 6-DoF sensing capability, along with on-board regulation, battery management, and the MSP430 as a central controller/processor. However, this platform added the capability to use either the previously 
mentioned Bluetooth radio or a standard MicroSD card, interfaced over MMC, for flash storage. Resultantly this platform also featured an offload interface implemented over RS-485, a half-duplex, differential communications protocol otherwise similar to the RS232 serial standard.

The TEMPO 3.2 Flash (3.2F) system was favored largely for its ability to be used in un-tethered deployments, or those in which nodes remain in the field taking data, possibly without any communication to or from technical collaborators, for a longer period of time. As a result of this demand for less physical interaction with the nodes, a more elaborate, stand-alone firmware operating system, along with support for a custom file system was created for use with these new flash-based nodes.

About 50 TEMPO 3.2 nodes were produced and power-profiled as the first part of this thesis work. While the 3.2 Bluetooth (3.2B) devices performed remarkably similarly to their 3.1 predecessors, the $3.2 \mathrm{~F}$ node yielded significant power savings over the previous platform. By eliminating the nearly $50 \%$ of the power budget consumed by the Bluetooth module, and replacing it with a much lower-power flash-based storage module the $3.2 \mathrm{~F}$ node produced power savings of up to $63 \%$ over previous Bluetooth solutions.

Since previously the challenges of up-keep and manufacturing of the TEMPO system were viewed as non-critical, the 3.2 node suffered from many of the same expected challenges as it predecessor. High cost and assembly challenges, along with difficulty sourcing some parts which had reached end-of-life, created challenges for large-scale production of the system. In addition the demand for remote deployment resulted in demonstration of many of the weak-points in the un-thethered design and proved a significant challenge in developing robust firmware, resilient to failures in the field. However, without significant thought put into design for testability, developers often faced significant challenges when working to program or debug the nodes, with little to no access to the device hardware once it was cased and calibrated.
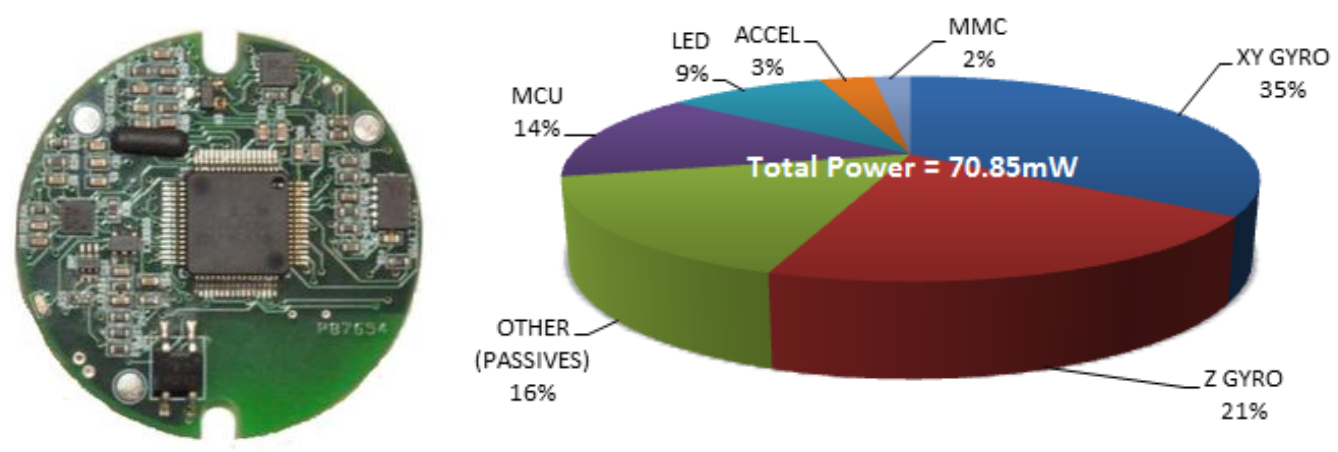

Figure 7: TEMPO 3.2 Node and Power Profile 
Though TEMPO 3.2 is still in the early phases of deployment, it has demonstrated the ability to collect 10 or more hours of data with all 6 DoF motion sensors active and up to 30 hours of data using only the accelerometers on the same $300 \mathrm{mAh}$ batteries used by the 3.1 system. Again, this has proved useful in longer-term studies where patients may wear a device home for several days, charging it each night. However, along with this demand comes the target for a reliable 12 plus hour battery life, while performing continuous $6 \mathrm{DoF}$ sensing. This opens the door into using the platform intelligently to extend battery life via MCU or interface (LED) based power reductions, as they now represent more significant portions of the power budget.

The conclusions of the TEMPO 3.2F development and deployment efforts support many of the claims introduced in the previous chapter. However, the TEMPO 3.2 system also demonstrates the unique challenge to producing a reliable, un-tethered device for body-worn operation, as inexact instructions and a more complex hardware-software ecosystem made the node difficult to deploy successfully. Nonetheless, those collaborators whose data collections did succeed using the TEMPO 3.2F system were happy with the extended battery life, and simpler data interfaces provided by the platform.

\subsection{Commercial Alternatives}

In the two years since development of the TEMPO 3.2 system was completed, a number of more recent commercial alternatives to the TEMPO platform have emerged. While some of these platforms target the same limited 3 or $6 \mathrm{DoF}$ application space as the previous system, others have began to target rapid extension into new, or possibly user-defined, sensing modalities. A brief survey of commercially available IMU products is provided in the following sections. This is intended both to familiarize the reader with the state-of-the-art in IMU platform design, and validate the assertion that no currently available platform achieves the stated goals of the TEMPO 4 system.

\subsubsection{YEI 3-Space IMU}

The YEI 3-space IMU represents the state-of-the-art in fully tethered USB-based motion capture solutions [19]. Because the platform operates only in a tethered, continuously reporting mode it is capable of both incredibly high fidelity signal capture, with a1.3kHz maximum raw sampling rate, and complex processing (on-node Kalman filtering with $385 \mathrm{~Hz}$ output). In addition it supports full $9 \mathrm{DoF}$, or simultaneous 3 axis accelerometer, gyroscope, and magnetometer motion capture, currently the gold standard for extracting information about motion and position in the global frame. 


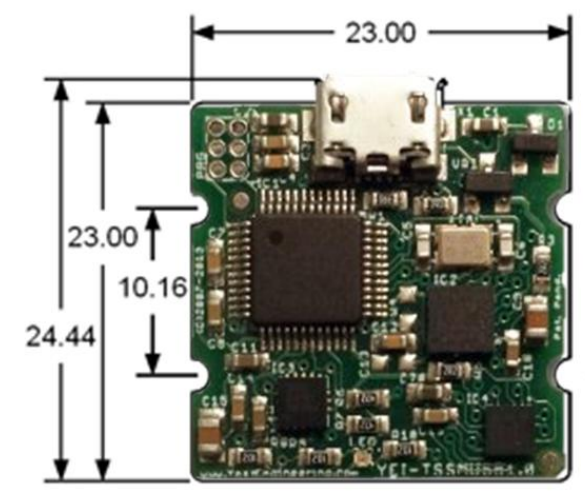

Figure 8: Dimensioned YEI 3-Space IMU (all dimensions in mm) [19]

The YEI 3-space IMU was selected as part of this market survey for two primary reasons. First, it demonstrates the usefulness of tethered IMU solutions in the ultra-high fidelity, and low wearability usecases. Second, it sets a commercial bar for small form-factor, low-weight IMUs, as without a battery, it measures just $23 \times 23 \times 2.2 \mathrm{~mm}$ and $1.3 \mathrm{~g}$.

\begin{tabular}{|c|l|}
\cline { 2 - 2 } \multicolumn{1}{c|}{} & \multicolumn{1}{c|}{ Value } \\
\hline Battery Life & Unlimited (tethered USB operation only) \\
\hline On-board Sensors & 3 axis accelerometer, gryo, and magnetometer (9 DoF) \\
\hline Interfaces & USB 2.0 \\
\hline Dimensions & $23 \times 23 \times 2.2 \mathrm{~mm}$ \\
\hline Mass & $1.3 \mathrm{~g}$ (no battery) \\
\hline Expandability & Serial interface (UART), custom software backend \\
\hline
\end{tabular}

Table 1: YEI 3-Space IMU Device Summary

\subsubsection{LPMS-B}

The LPMS-B is a research-compatible platform that represents a significant step forward in the development of powerful, high fidelity wireless motion capture. While also featuring 9 DoF sensing, with up to $300 \mathrm{~Hz}$ sampling rates, the LPMS-B offers up either Quaternion or Euler Angle pre-processed output and interfaces a PC over Bluetooth for data recovery and processing using custom code libraries and an open-source motion analysis toolkit [20].
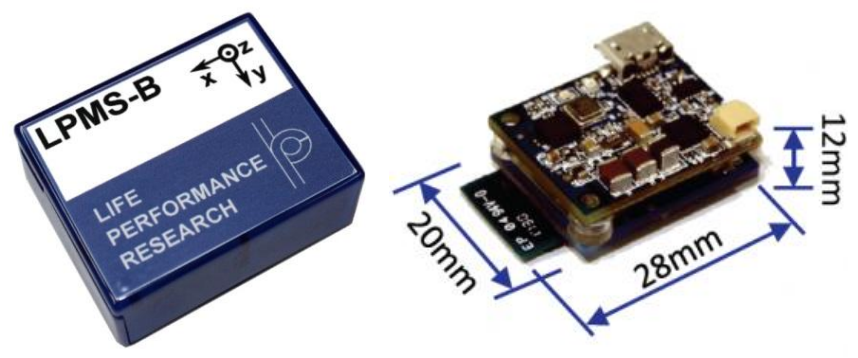

Figure 9: LPMS-B Platform [20] 
The LPMS-B platform was selected to be a part of this survey as it is a significant market competitor in the high-end, research-based, wirelessly aggregated space. In addition, it represents a highly integrated and tightly packaged device, in stark contrast with the more bare-bones YEI device presented above.

\begin{tabular}{|c|l|}
\cline { 2 - 2 } \multicolumn{1}{c|}{} & \multicolumn{1}{c|}{ Value } \\
\hline Battery Life & 10 hours (@800 mAh) \\
\hline On-board Sensors & 9 DoF, atmospheric pressure, and temperature \\
\hline Interfaces & Bluetooth 2.1, USB for charging \\
\hline Dimensions & $20 \times 28 \times 12 \mathrm{~mm}$ \\
\hline Mass & $34 \mathrm{~g}$ \\
\hline Expandability & None \\
\hline
\end{tabular}

Table 2: LPMS-B Device Summary

\subsubsection{X-IO X-IMU}

The $\mathrm{x}$-IMU platform is another $9 \mathrm{DoF}$ motion sensing platform offering up to $512 \mathrm{~Hz}$ system sampling rates. The platform is interfaced via USB, Bluetooth, MicroSD or a standard serial interface. In addition it provides several LEDs and an expansion port for interfacing with the user.
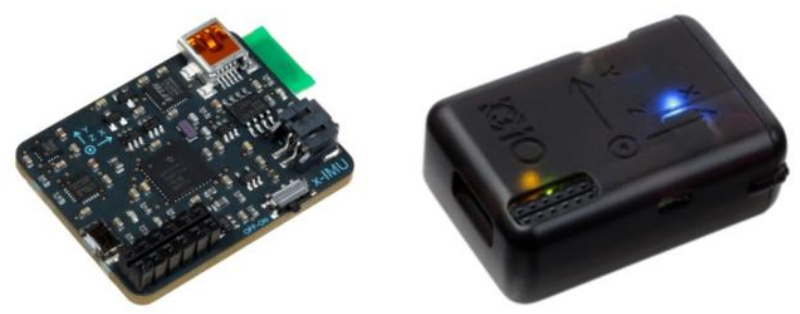

Figure 10: X-IO x-IMU Platform [4]

The $\mathrm{x}$-IMU was selected as part of this survey as it represents one of the only commercially available, IMU-specific platforms designed with a tightly integrated expandable interface. This interface includes 8 analog input or digital input/output pins, 4 pulse-width modulation (PWM) outputs, and a single Universal Asynchronous Receiver Transmitter (UART) interface capable of operating up to 1Mbaud. This makes it a principle market competitor to TEMPO 4 in the expandable IMU product-space.

Unfortunately, this interface primarily offers up simple analog or digital pins for signal capture or control, rather than the slightly more complex serial interfaces implemented as part of this work.

\begin{tabular}{|c|l|}
\cline { 2 - 2 } \multicolumn{1}{c|}{} & \multicolumn{1}{c|}{ Value } \\
\hline Battery Life & 6 hours (@ 300mAh) \\
\hline On-board Sensors & 9 DoF \\
\hline Interfaces & Bluetooth 3.0, USB, MicroSD \\
\hline Dimensions & $33 \times 42 \times 10 \mathrm{~mm}$ (no casing) \\
\hline Mass & $49 \mathrm{~g}$ (w/ battery) or 12g (w/o battery) \\
\hline Expandability & $3.3 \mathrm{~V}$ supply, 8 A/D IO, 4 PWM, and 1 UART \\
\hline
\end{tabular}

Table 3: x-IMU Device Summary 


\subsubsection{Shimmer 3}

The Shimmer platform has gained a great deal of attention lately for its wide-spread use in a number of academic deployments. By enabling research collaborators to quickly develop signal processing efforts on top of a core data reporting platform with a diverse set of biosignal-oriented add-on boards, the Shimmer 2 system found reasonable success in the low-budget preliminary case study market-space. However, the burdensome firmware operating model of the previous platform, and higher demand for greater control of platform operation has pushed the newer Shimer 3 system toward simpler software interfaces and a more bare-bones firmware operating model [5].
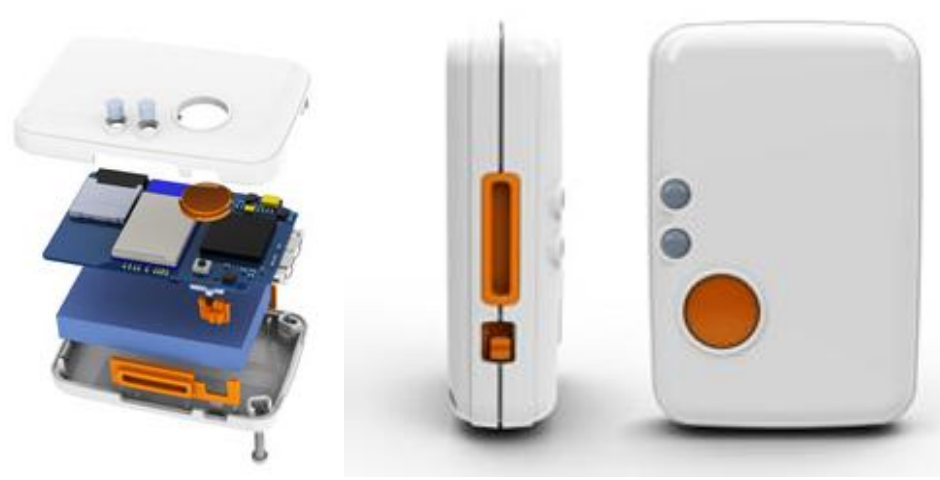

Figure 11: Shimmer 3 Platform [5]

The Shimmer 3 platform is considered as part of this survey for three primary reasons. First, it demonstrates the demand for an open, widely available, wearable device for on-body biosignal monitoring. Second, its predecessor platform demonstrates the limited, but increasingly present need for open hardware as well as firmware models. Last, but not least, this newly released, research-based, stateof-the art platform makes use of a very similar internal hardware architecture to the final TEMPO 4 node, making it a good point for comparison for base-level functionality.

\begin{tabular}{|c|l|}
\cline { 2 - 2 } \multicolumn{1}{c|}{} & \multicolumn{1}{c|}{ Value } \\
\hline Battery Life & Application dependent (@ 450mAh) \\
\hline On-board Sensors & 9 DoF, atmospheric pressure \\
\hline Interfaces & Bluetooth 3.0, MicroSD, Custom Dock \\
\hline Dimensions & $51 \times 34 \times 14 \mathrm{~mm}$ \\
\hline Mass & $20 \mathrm{~g}(\mathrm{w} /$ battery) \\
\hline Expandability & Internal and external development headers \\
\hline
\end{tabular}

Table 4: Shimmer 3 Device Summary

\subsubsection{Actigraph wGT3X-BT Monitor}

The wGT3X-BT monitor is a state-of-the-art motion monitoring-specific platform intended for use in activity and sleep monitoring. In addition to providing basic 3 DoF accelerometer sensing it also includes a light sensor to capture additional information about activity during the nighttime hours [21]. 

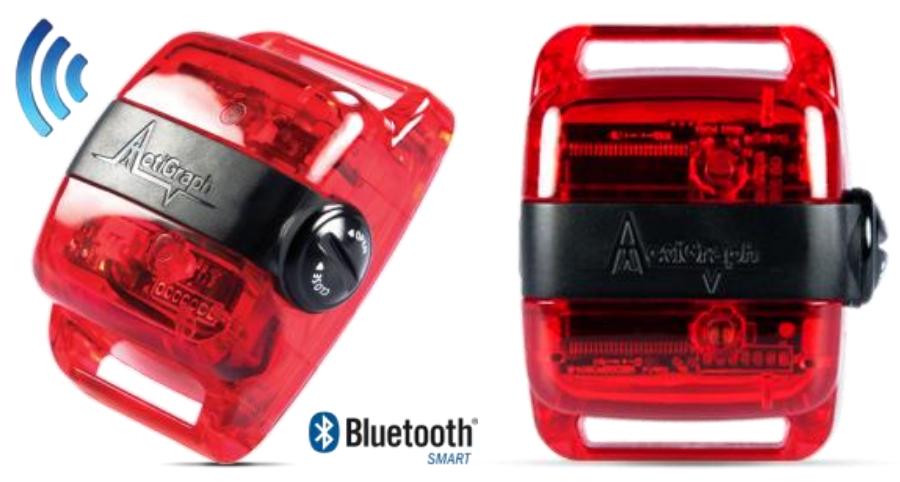

Figure 12: Actigraph wGT3X-BT Platform [21]

This Actigraph platform differs from some of the fuller-featured research-based platforms presented earlier in this section as it sacrifices some of the higher-fidelity measurement produced by these platforms in the name of significant increase of battery life and storage capacity. Thus, the wGT3X-BT device is included in this survey as it demonstrates a successful exploitation of the trade-off between sensing complexity and system lifetime.

\begin{tabular}{|c|l|}
\cline { 2 - 2 } \multicolumn{1}{c|}{} & \multicolumn{1}{c|}{ Value } \\
\hline Battery Life & 25 days (@ 800 mAh) \\
\hline On-board Sensors & 3 DoF (accelerometer only) and ambient light \\
\hline Interfaces & Bluetooth Low-Energy and USB charging \\
\hline Dimensions & $46 \times 33 \times 15 \mathrm{~mm}$ \\
\hline Mass & $19 \mathrm{~g}$ (w/ battery) \\
\hline Expandability & None \\
\hline
\end{tabular}

Table 5: Actigraph wGT3X-BT Device Summary

\subsubsection{FitBit Products}

One of the most significant players in the quantified-self and personal fitness domains of wearable electronics today is FitBit. Currently FitBit offers up three pedometer-based platforms, with varying levels of information and interfacing available. Rather than summarize each of these devices individually information regarding all three is included below.

\section{FitBit Zip}

The FitBit Zip represents the smallest form-factor, least fidelity, and lowest cost product manufactured by the company. Most importantly, with a non-rechargable CR2025 $160 \mathrm{mAh}$ battery and an approximate battery life of 4-6 months the Zip is included in this survey as it is by far the lowestpower device included in this survey.

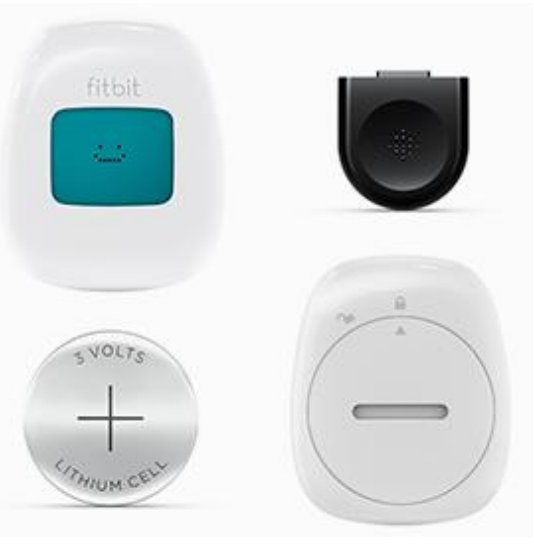

Figure 13: FitBit Zip Platform [3] 


\begin{tabular}{|c|l|}
\cline { 2 - 2 } \multicolumn{1}{c|}{} & \multicolumn{1}{c|}{ Value } \\
\hline Battery Life & $4-6$ months (@ 160 mAh) \\
\hline On-board Sensors & 3 DoF (accelerometer only) \\
\hline Interfaces & Bluetooth 4.0 and LCD \\
\hline imensions & $35.5 \times 28 \times 9.7 \mathrm{~mm}$ \\
\hline Mass & $8 \mathrm{~g}$ (w/ battery) \\
\hline Expandability & Back-end app development \\
\hline
\end{tabular}

Table 6: FitBit Zip Device Summary

\section{FitBit One}

The FitBit One is the company's most mature product, with several highly reviewed, working revisions

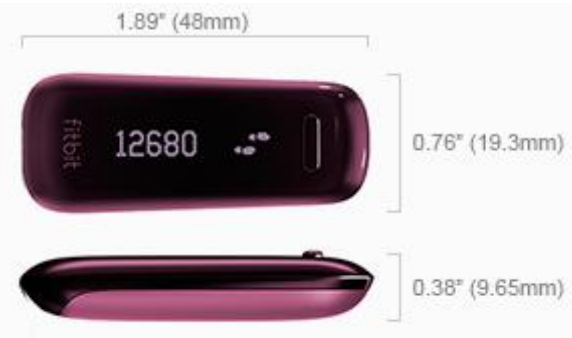

Figure 14: FitBit One Platform [22] under their belt. This device takes the form-factor of a typical clip-on pedometer and provides an impressive wireless recharging along with a nearly transparent wireless-in-range offload strategy. This device was included in this survey as it represents FitBit's most successful historical offering.

\begin{tabular}{|c|l|}
\cline { 2 - 2 } \multicolumn{1}{c|}{} & \multicolumn{1}{c|}{ Value } \\
\hline Battery Life & $5-7$ days (no capacity provided) \\
\hline On-board Sensors & 3 DoF (accelerometer only) \\
\hline Interfaces & Bluetooth 4.0 and LCD \\
\hline Dimensions & $48 \times 19.3 \times 9.7 \mathrm{~mm}$ \\
\hline Mass & $8 \mathrm{~g}$ (w/ battery) \\
\hline Expandability & Back-end app development \\
\hline
\end{tabular}

Table 7: FitBit One Device Summary

\section{FitBit Flex}

The FitBit Flex is the most recent addition to the line of products and represents FitBit's answer to the recent wave of wrist-worn IMU monitoring devices intended for both user motion capture and interfacing when connected to a smartphone. Following the trends of many significant market competitors the FitBit Flex integrates 3 DoF sensing, a vibrational motor, and a similar wireless charging interface to its cousin, the FitBit One, to

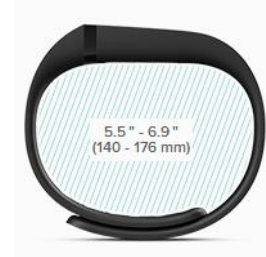

SMALL

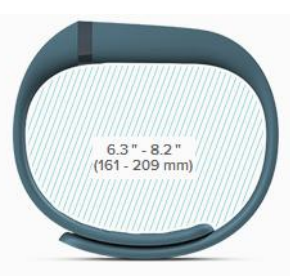

LARGE

Figure 15: FitBit Flex Platform [23] accomplish similar overall system specifications.

\begin{tabular}{|c|l|}
\cline { 2 - 2 } \multicolumn{1}{c|}{} & \multicolumn{1}{c|}{ Value } \\
\hline Battery Life & 5 days (no capacity provided) \\
\hline On-board Sensors & 3 DoF (accelerometer only) \\
\hline Interfaces & Bluetooth 4.0, LEDs, and motor \\
\hline Dimensions & Wristband (N/A) \\
\hline Mass & $?$ \\
\hline Expandability & Back-end app development \\
\hline
\end{tabular}

Figure 16: FitBit Flex Device Summary 


\subsubsection{Jawbone Up}

Last but not least, this work will introduce one of the significant market competitors to the FitBit Flex in order to better understand the state of the wrist-worn BSN design space. The Jawbone Up is another commercially popular and successful 3 DoF monitoring platform, sporting several indicating LEDs and a vibrational motor for user alerts. Its intuitive mechanical design, open back-end libraries, and relatively sleek formfactor make it a strong contender, but some would argue its lower level of integration, and non-health specific focus has cost it some success in the market. Nonetheless, the Up's high level of integration and impressive use

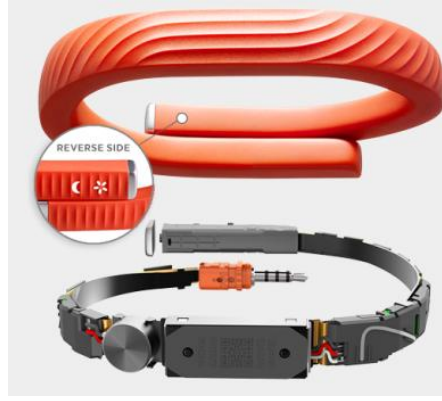

Figure 17: Jawbone Up Platform [24] of an incredibly low-capacity battery for long-term operation make it a platform of significant interest for this market survey.

\begin{tabular}{|c|c|}
\cline { 2 - 2 } \multicolumn{1}{c|}{} & Value \\
\hline Battery Life & 7 days (@ 32mAh) \\
\hline On-board Sensors & 3 DoF (accelerometer only) \\
\hline Interfaces & Bluetooth 4.0, LEDs, and motor \\
\hline Dimensions & Wristband (N/A) \\
\hline Mass & 19g \\
\hline Expandability & Back-end app development \\
\hline
\end{tabular}

Table 8: Jawbone Up Device Summary

\subsection{Product Summary}

This section presents a brief product summary comparing all of the platforms surveyed as part of this chapter and demonstrating their strengths and weaknesses in a side-by-side context. The intent of this table is to demonstrate the inability of any one platform to meet the demand for a low-power, long lifetime, flexible system for on-body IMU and non-IMU based deployments. In addition, rather than compare the surveyed devices in reliability, as this can often be difficult to gauge amongst competing commercially available devices, the table below considers built-in sensing diversity instead.

This consideration of on-board sensing modalities is designed both to give a measure of the ability of the system to target a wide range of applications based upon the on-node hardware and also to provide some idea of what portion of the underlying hardware and firmware design is committed to sensing components and code-structure, giving a better sense of both the specificity and flexibility of the platform. 
The following abbreviations are used in this table to indicate various sensing modalities, interfaces, and development strategies:

9 DoF: 3 axis accelerometer, gyro, and magnetometer human motion monitoring

6 DoF: 3 axis accelerometer and gyro human motion monitoring

3 DoF: 3 axis accelerometer human motion monitoring

Alt: Altimeter sensing, often via barometer

Temp: Ambient temperature sensing

Light: Ambient light (lux) sensing

D/A I/O: Digital or analog input or output

PWM: Pulse-width modulation output

UART: Universal asynchronous serial interface

Vib: Vibration output via motor

App Dev: Open application (often smart-phone driven) development

\begin{tabular}{|c|c|c|c|c|c|c|}
\hline \multirow{2}{*}{ Platform } & \multirow{2}{*}{ Lifetime } & \multicolumn{2}{|c|}{ Form-factor } & \multirow{2}{*}{ Interfaces } & \multirow{2}{*}{$\begin{array}{l}\text { Built-in } \\
\text { Sensing }\end{array}$} & \multirow{2}{*}{ Flexibility } \\
\hline & & $\begin{array}{c}\text { Volume } \\
\left(\mathrm{mm}^{3}\right)\end{array}$ & $\begin{array}{l}\text { Weight } \\
(\mathrm{g})\end{array}$ & & & \\
\hline YEI 3-Space IMU & $\begin{array}{c}\text { Infinite } \\
\text { (Tethered) }\end{array}$ & 1163.8 & $\begin{array}{c}1.3 \\
\text { (no batt) }\end{array}$ & USB & $9 \mathrm{DoF}$ & $\begin{array}{l}\text { App Dev, } \\
\text { UART }\end{array}$ \\
\hline LPMS-B & $\begin{array}{c}10 \mathrm{~h} \\
(800 \mathrm{mAh})\end{array}$ & 6720 & 34 & Bluetooth 2.1 & $\begin{array}{c}9 \mathrm{DoF}, \\
\text { Alt, Temp }\end{array}$ & App Dev \\
\hline X-IO x-IMU & $\begin{array}{c}6 \mathrm{~h} \\
(300 \mathrm{mAh})\end{array}$ & 13860 & 49 & $\begin{array}{l}\text { USB, Bluetooth } \\
\text { 3.0, MicroSD, } \\
\text { LEDs }\end{array}$ & $9 \mathrm{DoF}$ & $\begin{array}{l}8 \text { D/A IO, } \\
\text { 4 PWM, } \\
\text { UART }\end{array}$ \\
\hline Shimmer 3 & $\begin{array}{l}\text { Application } \\
\text { Dependent } \\
(450 \mathrm{mAh})\end{array}$ & 24276 & 20 & $\begin{array}{l}\text { Bluetooth 4.0, } \\
\text { MicroSD, } \\
\text { LEDs, Dock }\end{array}$ & $9 \mathrm{DoF}$, Alt & $\begin{array}{l}\text { JTAG, } 2 \\
\text { expansion } \\
\text { headers } \\
\end{array}$ \\
\hline $\begin{array}{c}\text { Actigraph WGT3X- } \\
\text { BT }\end{array}$ & $25 \mathrm{~d}$ & 22770 & 19 & $\begin{array}{l}\text { USB, BLE, } \\
\text { LEDs }\end{array}$ & $\begin{array}{l}3 \mathrm{DoF}, \\
\text { Light }\end{array}$ & None \\
\hline FitBit Zip & $\begin{array}{c}4-6 \mathrm{mo} \\
(160 \mathrm{mAh})\end{array}$ & 9592.1 & 8 & $\begin{array}{l}\text { Bluetooth } 4.0, \\
\text { LCD }\end{array}$ & $3 \mathrm{DoF}$ & App Dev \\
\hline FitBit One & $5-7 d$ & 8939 & 8 & $\begin{array}{l}\text { Bluetooth 4.0, } \\
\text { LCD }\end{array}$ & $3 \mathrm{DoF}$ & App Dev \\
\hline FitBit Flex & $5 d$ & Wristband & $?$ & $\begin{array}{l}\text { Bluetooth 4.0, } \\
\text { LEDs, Vib }\end{array}$ & $3 \mathrm{DoF}$ & App Dev \\
\hline Jawbone & $\begin{array}{c}7 \mathrm{~d} \\
(32 \mathrm{mAh})\end{array}$ & Wristband & 19 & $\begin{array}{l}\text { Bluetooth 4.0, } \\
\text { LEDs, Vib }\end{array}$ & $3 \mathrm{DoF}$ & App Dev \\
\hline TEMPO 3.1 & $\begin{array}{c}4-6 \mathrm{~h} \\
(300 \mathrm{mAh})\end{array}$ & Wristband & 40 & $\begin{array}{c}\text { Bluetooth 3.0, } \\
\text { LEDs, Dock }\end{array}$ & $\begin{array}{l}6 \mathrm{DoF}, \\
\text { Temp }\end{array}$ & $\begin{array}{c}\text { HW/FW/SW } \\
\text { Dev }\end{array}$ \\
\hline TEMPO 3.2 & $\begin{array}{c}10-30 \mathrm{~h} \\
(300 \mathrm{mAh})\end{array}$ & Wristband & 40 & $\begin{array}{l}\text { Bluetooth } 3.0 \text { or } \\
\text { MicroSD, } \\
\text { LEDs, Dock }\end{array}$ & $\begin{array}{l}6 \text { DoF, } \\
\text { Temp }\end{array}$ & $\begin{array}{c}\text { HW/FW/SW } \\
\text { Dev }\end{array}$ \\
\hline
\end{tabular}

Table 9: Surveyed Commercial Alternative Market Comparison 


\section{Chapter 3}

\section{Co-Design Concepts and Subsystem}

\section{Designation}

This chapter addresses two of the primary challenges to the stated contributions of this work. Namely these are designing hardware and firmware in the application-uninformed context and effectively partitioning system design methodology into smaller subsystems for iterative development and testing.

\subsection{Co-design Introduction and Case Studies}

The term co-design can often mean many things to many people with varied perspectives on a system design space. For this purpose the term will be defined explicitly for use in this work as follows.

Co-design is the process of using cross-hierarchical (i.e. hardware, firmware, and application layer) information in evaluating trade-offs which may have otherwise seemed arbitrary, irrelevant, or unintuitive from a non-system level designer's perspective.

It is understood that the definition above is somewhat vague, and intentionally so, as to capture the full scope of co-design in the context of this work. In order to make this definition somewhat more concrete, and also provide some examples of non-traditionally considered trade-offs eluded to previously, two case studies are presented in the remainder of this sub-section. The first study demonstrates how a selection typically made by a "hardware" designer, might deeply impact reliability and robustness of firmware operation, and present hurdles to the open development community. The second case study demonstrates a more traditional firmware trade-off and why it is approached by various designers in various ways, taking the system-level perspective on the challenges and benefits posed by two fundamental control strategies.

\subsubsection{Co-design Case Study 1: USB Transceiver Selection}

The topic of USB transceiver selection is typically one of hardware footprint, power delivery, routing considerations, and Application Specific Integrated Circuit (ASIC) solutions. Though firmware designers may be brought into the process to assure that USB communication will in fact be possible given a 
prescribed software operating model, the common perspective in the development field is that heavy use of hardware peripherals and software libraries largely masks away the complexity of coordinating complex, high speed interactions, such as those that take place in USB interfaces.

As a part of the preliminary hardware surveys conducted for the TEMPO 4 system, the topic of USB transceiver selection took center-stage. Since the TEMPO 4 platform targets all wired charging and interfacing to a single on-board USB port, analysis of reliable COTS products for USB operation was considered thoroughly. As a result of this survey three primary candidate solutions emerged, these are presented below.

\section{Candidate 1: MCU Driven Solutions}

Several MSP430 5xxx series

microcontrollers from Texas Instruments feature an integrated hardware peripheral for USB 2.0 communication. It just so

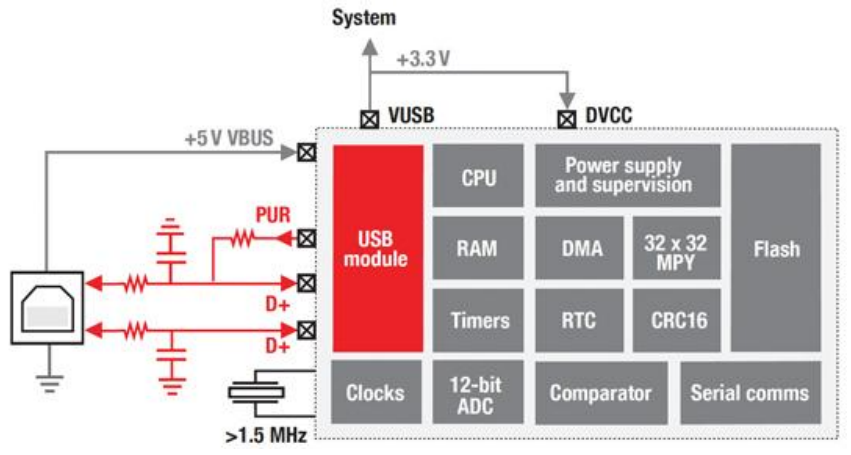

Figure 18: TI MSP430 w/ Integrated USB Transceiver [25] happened that this was also the family of controllers already being considered for use in this project. In addition a number of other MCU devices have had firmware libraries written to perform software-driven USB operation if clocked appropriately. As a result the possibility of an all in-MCU USB solution was considered both for its low form-factor and high expandability considerations.

\section{Candidate 2: High-speed ASIC Solutions}

A number of manufacturers provide ASIC topologies designed to contain one or several memory mapped USB end-points for communication. These transceiver circuits feature the necessary on-chip oscillators and decoder structures for reading and writing USB 2.0 or 3.0 data in full-speed and high-speed modes. Typically these chips feature high-pin count and a number of offload interfaces for streaming data in and out in serial or parallel formats. Thus high-speed ASICs were considered both for flexibility and robustness.

\section{Candidate 3: Low-speed ASIC Solutions}

In addition to the larger, more powerful high-speed ASICs, designed for full-speed USB communication, a number of popular manufacturers, such as FTDI, produce lower-speed USB 2.0 transceiver ASICs that allow the user to interface USB with lower-pin count, and often easier to access, serial solutions. These transceiver solutions were considered based on form-factor, ease of interfacing, and robustness. 
In discussing the trade-offs between these three candidate approaches co-design concepts will prove invaluable. At first glance, the hardwareonly solution to this problem seems straight forward; the MCU-driven solution saves cost, area, and routing complexity by reducing part count and improving level of integration.

In taking this initial hardware-driven perspective, an MSP430 prototype platform, designed to implement and test USB functionality integrated into the MSP430F5510 MCU, was purchased and

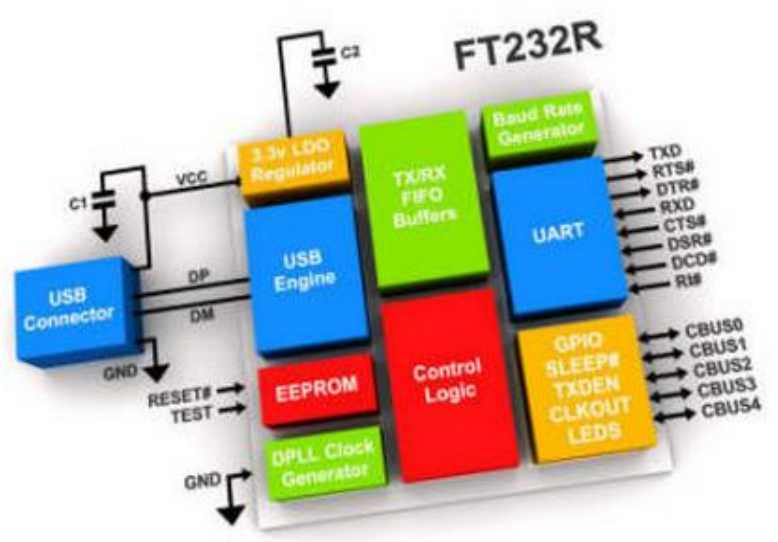
example libraries were downloaded from TI. The

Figure 19: Block Diagram of FT232 Low-speed USB Transceiver IC [26] code was then compiled and loaded onto the platform in order to evaluate system performance.

Even before beginning evaluation it became clear that this solution would not be ideal from a firmware operational perspective. To begin with, USB transceiver operation requires at least a $12 \mathrm{MHz}$ clock, which would need to be sourced within the MSP itself. This represented a challenge as typical core clock rates for previous TEMPO system have been in the $<4 \mathrm{MHz}$ range, meaning sourcing a $12 \mathrm{MHz}$ oscillator to the USB would result in tripling average system power during runtime. In addition it was nearly immediately noticed that the compiled size of the USB transceiver code was almost half of the $10 \mathrm{kB}$ code limit prescribed by the freeware version of Code Composer Studio available to the public. This meant that by implementing an on-MCU USB solution the platform would in fact save area, but also pay a significant power and performance cost along with way, limiting both the range of configurable system clock rates and the amount of user-defined code that could be implemented on the platform. Thus ASIC-driven options were considered to reduce code-complexity, and MCU clocking constraints.

The dilemma of high versus low-speed ASICs is another in which consideration of co-design concepts can be ciritical. Again, a more traditional perspective might opt in favor of a lower-footprint, higherspeed ASIC over a slower one with a possibly simpler host-controller interface. Again pursuing this hardware-only driven perspective, a lowfootprint, high-speed transceiver ASIC from FTDI, the FT121 was considered. Again a development platform with the ASIC on it was

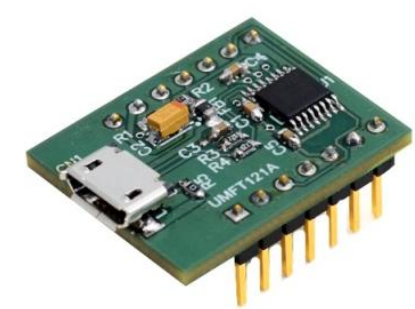

Figure 20: FT121 SPI-driven ASIC Evaluation Module [27] 
purchased and evaluated.

Though the FT121 did require much less firmware to configure its end-point control registers, it was quite complex to go about configuring and receiving on an end-point packet as a common developer. Also, though the chip did offer up a programmable interrupt line, it was deemed too complex for an enduser to manage in his/her own application-level firmware code. Thus, a special purpose library to handle communication with the FT121 ASIC would need to be created. For reasons to be discussed in the following chapters, this is not a good fit to the firmware communications operating model adopted by the TEMPO 4 system. In addition, the MCU-side storage for device configuration register values and added complexity of software-side USB endpoint management means more operational obfuscation, and less room for develops to quickly innovate on top of the hardware platform.

The final decision regarding the USB transceiver solution used in this work may in fact be the least intuitive from a firmware-agnostic perspective. A slightly higher pin count (i.e. larger form-factor), higher power, and less controllable transceiver circuit, the FT232 from FTDI, was selected. The reasons for this selection are varied and stretch from the simplified hardware interfacing model all the way up to the easeof-use for high-level application developers.

One primary motivation for selection of the FT232 USB transceiver was that it is one of the few Integrated Circuits (ICs) offering up direct USB to UART translation, making it a standard digital interface as seen by the MCU. The ability to read and write this interface using libraries already produced for peripheral communication results in reduced firmware bloat and provides an easy-to-conceptualize asynchronous digital interface to the application developer. Meanwhile, adoption of the FT232 into the custom docking station for the previous TEMPO 3.2 platforms meant that a software backend, written in Python, had already been established to communicate with these ICs over USB.

From the traditional hardware perspective, many electrical considerations, such as power consumption or input voltage range are avoidable when considering the FT232, as it has its own on-chip regulators to produce the $3.3 \mathrm{~V}$ supply its internal circuits operate on from the USB $5 \mathrm{~V}$ input. This puts the device in what is referred to as "self" or "bus" powered configuration. Since the transceiver's operation is only important when the USB is actually plugged in this works to reduce the management overhead affiliated with power gating the chip, and also provides an additional level of electrical isolation between the USB and system power domains.

In addition to power loss, there is also the question of area overhead introduced by the device. Here, we must look across only a hardware-boundary to realize that even in the worst-case FT232 layout only about 
$50 \%$ of the total USB area consists of this slightly larger USB transceiver IC and affiliated circuitry. In other words, regardless of how much the transceiver circuitry is compacted, total area will always be bounded below by the size of the connector itself. Thus, for the purpose of this work, it was deemed that the $10-40 \%$ of USB area savings achievable using a lower footprint, more customized part, or an MCU-based USB solution would not justify the significant increase in firmware overhead, and potential for reliability and flexibility issues related to this custom management code.

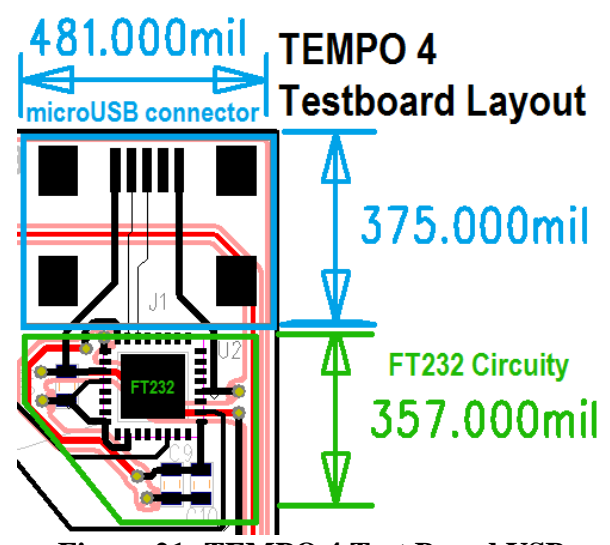

Figure 21: TEMPO 4 Test Board USB Layout w/ Dimensioning of Connector and FT232 Circuitry

Before this case-study concludes, it will demonstrate one more, all-important principle in the area of simplified, ultra-low profile electronics: the ASIC manufacturer is always adapting to market demand. Between the time at which a final bill of materials for the TEMPO 4 platform was settled on and the time at which the final production design of the TEMPO 4 platform went out, FTDI introduced a new, lowerprofile version of the FT232, the FT230X. This device has all the functionality of its big brother, but with a reduced set of the programmable I/O pads located on the FT232 which are not used in this design. Unfortunately, limited availability of development boards for testing, and pressure to complete the final design of the TEMPO 4 platform resulted in this newer IC not being implemented in TEMPO 4.0. However, a TEMPO 4.1 revision should surely consider a prototype implementing this smaller form factor device.

\subsubsection{Co-design Case Study 2: Polled versus Interrupt- driven Operation}

A great deal of work in the system-level coding community focuses on

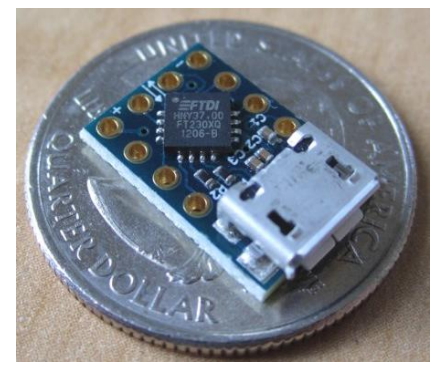

Figure 22: FT230x Development Board [28] the trade-offs and unique opportunities offered up by both polled and interrupt-driven operation. In the context of the previous TEMPO platform this section will provide one example of each type of operation and demonstrate why it is advantageous from the perspectives of system-level power reduction and increased firmware reliability.

\section{Benefits of Interrupt Driven Timing and Challenges of System Synchronization}

Asynchronous, interrupt-driven operation is typically the mode implied in many low power, low throughput applications where the use of wake-from-interrupt style operation offers tremendous reduction 
of overall system power. By running at high processing frequencies, and correspondingly powers, during wake periods and sleeping for large portions of inactive time, many systems that sample and process data infrequently can drive their average powers down to within an order of magnitude of that of sleep. When specialized circuit designs are coupled with these ultra-low power sleep states, this can mean significant reduction in average system power [29].

In the TEMPO 3 systems, all timing routines were interrupt-driven. This meant that sampling timers, along with system clock-keeping was all handled asynchronously from the operating code. This presents a challenge in-and-of itself, as the synchronous executed code, or that code which runs from the MSP's instruction memory, is not made aware of the execution of these asynchronous routines. To reconcile this runtime synchronization issue, an event-queue structure was created by the system designers. This queue allowed for passing of messages between the pool of asynchronous interrupts monitoring sampling and system time and the synchronous execution of the core, which of course took place whenever an interrupt was not in service. It is worth noting that any interrupt on the MSP430 used in the TEMPO 3 systems takes approximately 7 cycles to call and 7 cycles to respond from, so some timing slack is introduced by this interrupt-driven operation, but since this call and response time is expected to be constant it can typically be corrected for in the runtime code.

This asynchronously-driven event-queue model allowed the designers to exploit the power benefits of having the system asynchronously managed during idle periods, while still achieving significant throughput and computational ability during active periods such as on-node compression or Bluetooth transmission. In addition to power benefits, the model provides a relatively easy-to-use interface for those seeking to develop both additional computational and interrupt-driven libraries for the device. As will be mentioned later in this work, this core operating principle is considered robust enough that the TEMPO 4 system firmware is still based upon an event-driven execution scheme.

\section{Effective Uses and Drawbacks of Polling for Low Power Operation}

Polling operation is less common to find being used in low power systems. Rather than focus on the obvious misuses of polled operation, such as constantly monitoring an interrupt flag via synchronous code or simply looping on a null instruction rather than using sleep to create delay, this section will attempt to highlight some of the effective areas and challenges for polling-driven operation in a system-level context.

In the TEMPO 3.1 node, all data communication was accomplished in the form of polling from the aggregator-side. By exploiting the increased processing power, and available Bluetooth stack on most 
PCs and smar phones, this allowed the TEMPO 3.1 system to keep its own firmware control of the radio rather minimal. In addition, it allowed mid-sized ( $<8$ node) star-topology networks to be established fairly easily, as each node was polled by the master using a simple round-robin approach.

The TEMPO 3 node's Bluetooth module operates based around an asynchronous UART connection, so an interrupt is sourced in the MCU whenever data is being received from the radio. This enables a rather simple scheme of communication: the master (PC) pairs with the node (slave) over Bluetooth then, when ready, sends a start of session command telling the node to start taking data. Once the node is taking data the master then polls each node once a second to retrieve all its captured data. Since the nodes can buffer 2-3 seconds worth of data at a time, so long as the master requests each slave's data once per second or so, the PC was sure to have received a time-continuous stream of data. Otherwise a circular buffer was used to store data on-node assuring the device would report only the most recent $2-3 \mathrm{~s}$ of sample values when eventually polled again.

This polled data collection technique lent itself well to the event-queue driven operation of the TEMPO 3.1 system as it allowed developers to add any amount of data they would like to the out-going buffer before the node was polled again by the master. By creating this wireless tether between the PC and the node, the designers were also able to accomplish significant reduction in firmware complexity, slaving all operations to the command of the Bluetooth interface at the expense of a large portion of the hardware power-budget being consumed by the affiliated hardware module.

One potential radio power benefit of this approach is that, without a polling command received from the master the node does not attempt to transmit any data. This would, given a more efficient radio communication strategy, present the potential for significant power savings from a mostly-listen or asynchronous, bursty transmission protocol. Unfortunately, the TEMPO 3.1 system was not able to benefit from this added feature of its communication control scheme as Bluetooth is a Time-Domain Multiple Access (TDMA) protocol which has nearly symmetric transmit and receive buffer power.

Had the designers implemented an asynchronous interrupt-driven wireless data communication scheme instead, care would need to be taken on both the master side, to assure there was in fact room/time in the schedule for a new node, and by the slave, to monitor for when/where the transmission should be made to avoid collision. This would likely result in an increase in the overall system power and firmware complexity. 


\subsubsection{Co-design Goals and Conclusions}

The co-design concept stressed here is that of ease-of-interfacing. When simplistic interfaces, well suited to the underlying operation of the application to which they are paired, are used effectively they can produce significant gains at both the hardware and software level simultaneously. The effort affiliated with this work's general contributions is exactly this, to identify opportunities for cross-hierarchical optimizations in today's changing design space.

\subsection{Subsystem Designation}

The following 3 chapters of this document are structured around the development of three principle subsystems, that when working together, comprise the entirety of the TEMPO 4 platform functionality. This work intentionally partitions itself along the lines of functional subsystems, rather than the traditional hardware, firmware, and software divisions in order to exploit the co-design opportunities for cross-hierarchical optimization introduced in the previous section of this chapter.

The basic concept of this organizational view is demonstrated in Figure 23. By taking a traditional design challenges, such as implementing an application programmable timer or SPI interface and performing tasks from hardware selection all the way through application coding iteratively throughout the design process, the implications of application information on possible system-level hardware decisions are better clariefied and understood.

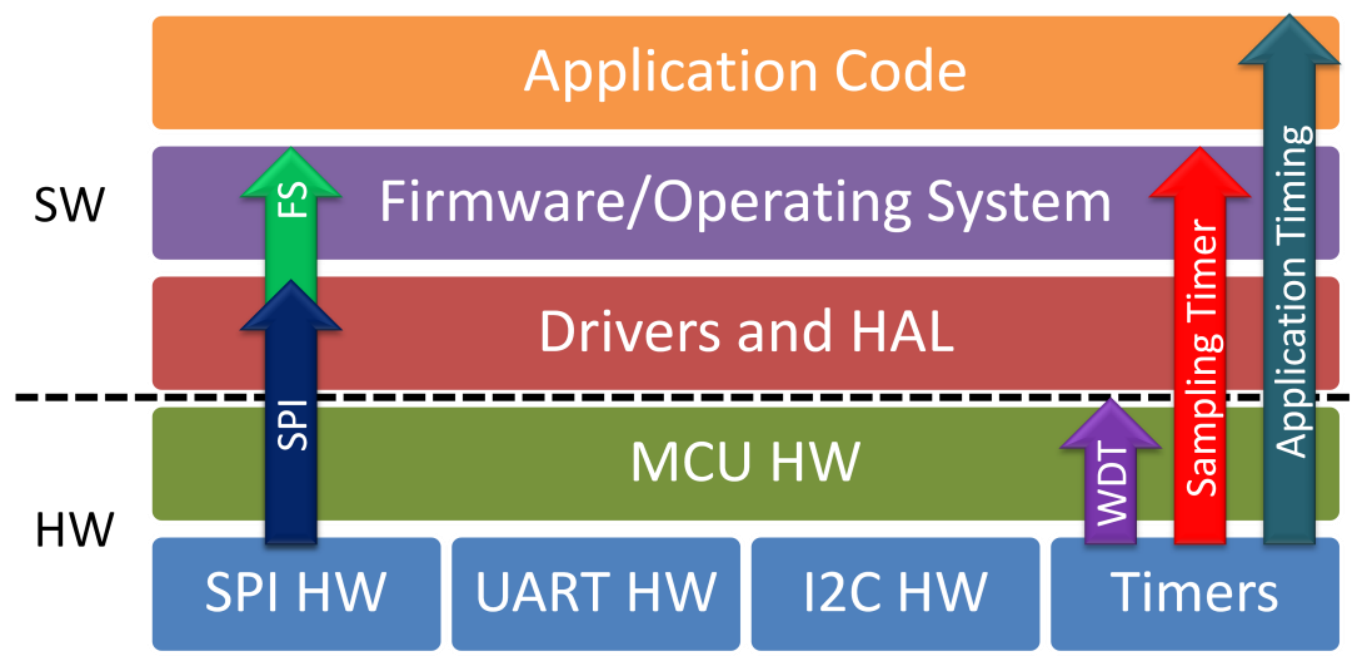

Figure 23: Cross-hierarchical Development Model

For the sake of simplicity this work will establish three principle areas of contribution to the co-design of hardware and firmware IMU solutions for the body-worn context. These areas are hereby referred to as 
subsystems as they designate functional, vertically integrated system sub-components, rather than particular hardware, firmware, or software, inside of the TEMPO 4 operating model.

\section{Battery Management and Supply Regulation}

2. Programming, Control, and Interfacing

\section{Sensing, Storage, and Transmission}

The three subsystems introduced above are partitioned the way they are for several key reasons. To begin with battery management and supply regulation are of course tightly coupled concepts, but more importantly the significant decoupling of control and monitoring between the power delivery network and remainder of the system justifies this decision. Overall this is seen as a positive feature of the specified design as it means other subsystems will have to source relatively little control to the power delivery network, and will only be responsible for monitoring and conditioning of their own delivered power rails of interest.

Programming, control, and interfacing are grouped and separated from sensing, storage, and transmission as the former tasks involve the determination of what the system will be able to interface, while the later address the challenge of tapering this interface to fit any given deployment. To make this division more explicit consider the challenge of developing a sensing system with an unknown sensor requirement. Without critical information such as Nyquist rate or signal content, it is difficult to near impossible to specify the remainder of system operating parameters. For this reason the programming, control, and interfacing portion of this work borrows largely from past experience, current market direction, and commonly available standardized interfaces to attempt to provide a reasonably unconstrained environment in which to develop for new sensing, storage, and transmission platforms and media.

The following three chapters of this document will each address the design of one of the three subsystems introduced above. Each will attempt to address both the traditional hardware and firmware challenges posed by the space then explicitly discuss opportunities for co-design optimizations, and finally the solution arrived at for the TEMPO 4 platform. It is important to remember when reading the following chapters that this work followed and iterative hardware-firmware design process, wherein a piece of hardware was not accepted into the system design until it had been verified to perform with desired metrics in subsystem and system-level test benches. 


\section{Chapter 4}

\section{Battery Management and Supply}

\section{Regulation}

The issue of battery management and supply regulation is a primary one in the wearable design space.

The targeted metrics of form-factor and lifetime are called directly into question, and in the case of many common battery chemistries, can be traded off to demonstrate advantages of some non-traditional design decisions for ultra-low power wearable technology. This chapter will address the selection of battery chemistries and capacities for on-body deployments, discuss the challenges of battery charging and management techniques in the context of low form-factor designs, and finally address the issue of selecting a regulator topology for the ultra low-power, body-worn context. It concludes with a demonstration of important co-design concepts and a final design summary for the TEMPO 4 battery management and supply regulation design.

\subsection{Battery Chemistry and Capacity}

The challenge of specifying a battery chemistry and capacity for a cordlessly-powered system is a significant one as it affects nearly every targeted metric if conducted improperly. Form-factor and lifetime constraints implied by batteries can appear rather straight forward; however, the internal series resistance, voltage level at a battery's output, and the battery's ability to source large amounts of current over a short period of time, can impact reliability, ease-of-interfacing, and even flexibility in some cases. This section briefly discusses selection of a battery chemistry and capacity in a general context, by indicating trends in normalized metrics for various battery technologies.

\subsubsection{Form-factor versus Lifetime Constraints}

The obvious trade-off implied by battery selection is that of form-factor versus lifetime. As batteries grow larger, typically their capacity increases, not necessarily linearly with size. If an individual chooses to design a product implementing a standard-sized battery into the casing, this often means specifying a particular lifetime at a given physical size. Since this works attempts to target the widest possible range of system deployments it does not consider one particular battery size or package, but rather families of batteries, organized by the chemistry through which they produce electrical energy. This type of 
organization is useful as a battery's chemistry is directly correlated to both its ability to be recharged and the circuitry required for performing this recharging if possible.

Battery capacity is typically provided in milli-ampere hours (mAh) which does not take into account the differing nominal output voltages for various cell chemistries. In order to normalize out this voltage-level variation the energy-capacity of a cell is instead calculated by taking a scaled product of its capacity and nominal output voltage. The result is an energy capacity in Joules that can be measured relative to other dissimilar battery chemistries and form-factors on a level playing field. The underlying figure of merit to this model of battery chemistry analysis is that of energy-density, which explicitly provides the energy stored per unit volume in the battery. Energy-density is a common figure of merit in all forms of energy storage, but has various interpretations. For the purpose of this work, a purely volumetric energy-density was borrowed, using capacity in milli-amp hours, nominal cell voltage, and of course cell volume, to produce a simple figure of merit for evaluation. This metric is provided, with appropriate normalization to Joule per unit volume, below.

$$
E_{D}=\frac{3.6 * C_{m A h} * V}{\text { Volume }}
$$

As part of this work, a large variety of commonly available alkaline and lithium-based cells were considered for use. Preliminary determinations ruled out a large percentage of the commonly available, cylindrically packaged cells due to their large mass and volume. In addition the challenge of whether the system would be capable of obtaining a battery life acceptable for a one-time-use battery was also considered.

The first several iterations of the TEMPO 4 design called for use of a non-rechargeable coin-cell battery, located on the backside of the PCB, but as the design grew more complex, and power and area at a premium, it was determined that an off-board battery solution would be implemented. Both Lithium and Alkaline-based cells were considered as part of a market surbey. The results of this survey are summarized in the single-log plot of volume versus battery capacity, in Joules, for various chemistries and packagings provided in Figure 24 below. 


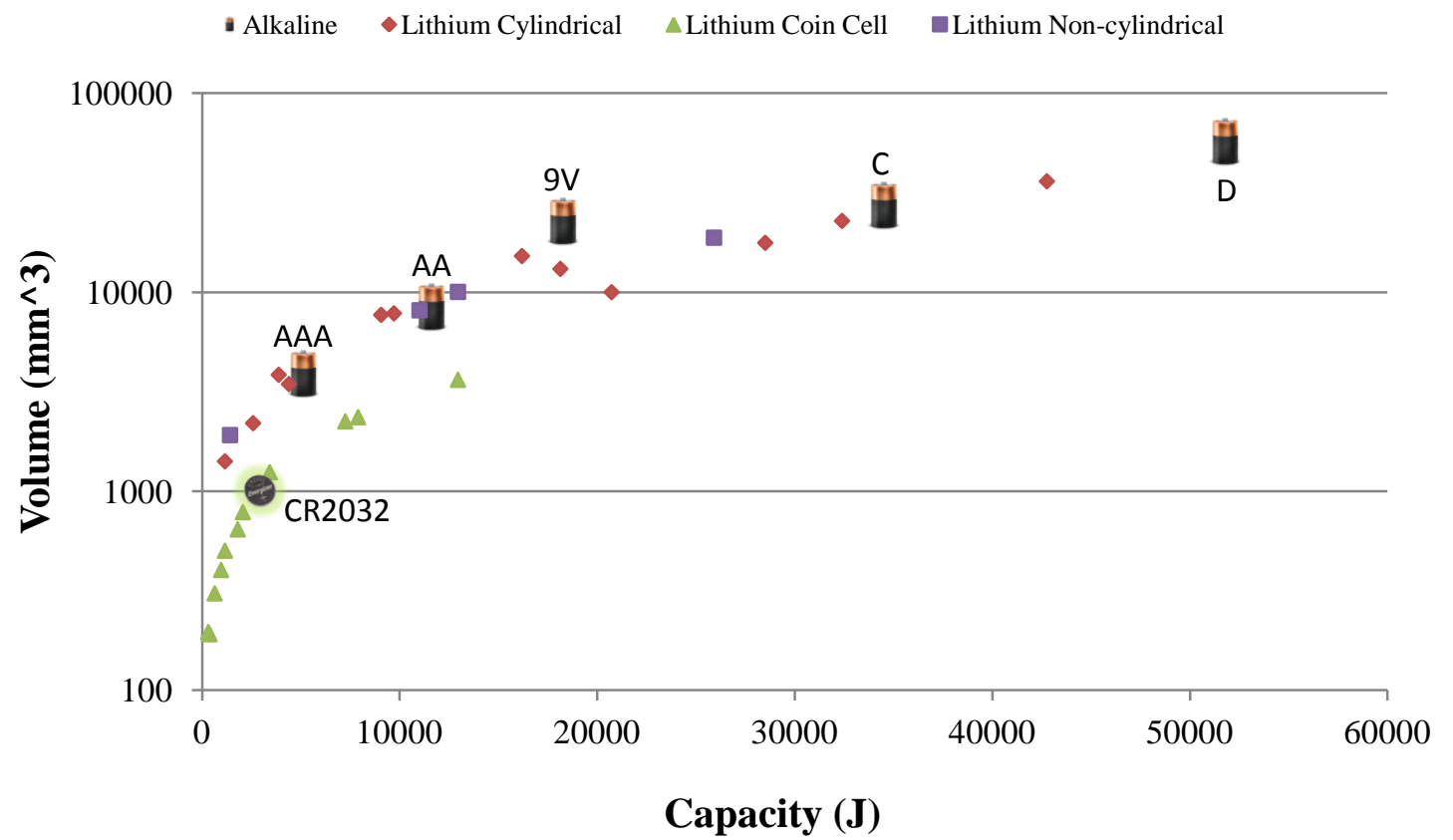

Figure 24: Battery Cell Capacity versus Volume for a Number of Chemistries

There are several important conclusions to be drawn from Figure 24. First, the low-volume, low-capacity portion of the design spectrum is dominated nearly entirely by lithium coil-cell topologies, both rechargeable and nonrechargeable. Once energy capacity exceeds the $10 \mathrm{~kJ}$ mark, the common alkaline and larger lithium cells begin to stand out. One interesting conclusion to be drawn from this plot is that energy density for alkaline cells falls just short of that of their newer lithium counterparts. What this plot does not show, are the significant mass benefits offered up by the packaging of some lithium ion (LiIon) and polymer (LiPo) batteries. While cylindrical lithium

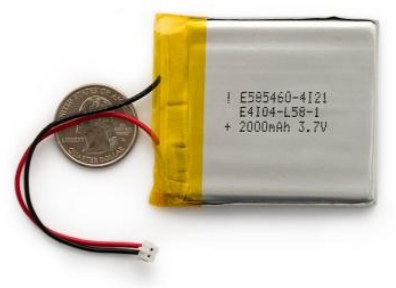
packages are similar in mass to their standardized alkaline counter-parts, alternative packaging for LiIon and LiPo cells makes this weight reduction possible. For example, while a 2000mAh thin package weighs $35 \mathrm{~g}$ [30] its nearest cylindrical lithium competitor weighs $45 \mathrm{~g}$ [31], or nearly $30 \%$ more.

Thus, in long-term deployments, where non-rechargeable batteries can provide acceptable lifetimes and the extra mass of their packaging is not an issue, it is still prudent to make use of standard packaged, cylindrical cells. Meanwhile, in similar lower-power scenarios where form-factor is a major issue, coin cells can produce acceptable battery life for some applications [3]. However, currently, the optimal tradeoff in energy capacity and a combined volume-mass metric are the thin-packaged lithium-based cells. 


\subsubsection{Application Considerations for Lithium Cells}

The use of lithium cell chemistry does call for some additional consideration of application-driven power constraints. It is well known that large instantaneous current draws can significantly shorten the lifetime of, and in some cases permanently damage, Lithium cells [32]. Thus, in order to avoid the long-term negative effects of such large instantaneous current draws, hardware designers attempt to use effective decoupling strategies to source larger instantaneous currents rather than relying on the battery alone. The challenge of storing enough energy on-node to prevent significant voltage dip and sag, and protect the cell is further discussed in the supply regulation portion of this chapter.

In regard to operational constraints implied by this poor suitability of Lithium cells to large instantaneous current draws, there are several considerations that cross the hardware-firmware boundary in this space. First and foremost, this implies Lithium-based energy storage is inherently poorly suited to bursty operation, as though the average power of many sleep-wake approaches is similar to their "always-on" counter-parts, the larger instantaneous draws from the battery implied by condensed operating time window may be damaging. This is one promising argument for lower power, lower frequency continuous operation over today's much more pervasive duty-cycled approaches.

From an embedded hardware designer's perspective there is little besides effectively decoupling components or providing an additional power source that can help to resolve these issues. However, from the firmware designer's perspective there are a number of considerations that can help alleviate unnecessary battery fatigue. First and foremost the use of lower clock speeds and less bursty operation can help to reduce large instantaneous current draws. In addition, high power operations such as flash writes/erasures or radio transmissions, should be spread out as much as possible as to allow decoupling capacitors to regain charge after being partly or fully depleted by the draw of an expensive operation.

\subsubsection{Battery Conclusions}

After much debate on ease of sourcing a battery and the desire to make the TEMPO 4 hardware accessible to all system developers, a general solution was arrived at. The TEMPO 4 platform supports a standard JST connector for battery interfacing, allowing for the use of a wide variety of potential battery options for the platform including rechargeable and nonrechargeable chemistries. In addition to allowing for the use of a wide

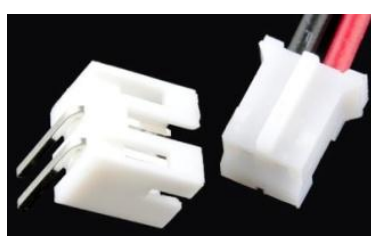

Figure 26: Standard JST Connector [33] 
variety of battery packs already terminated with JST connectors, this design decision also allows for both soldered-lead connections as well as adapters for converting other battery termination styles into the widely available JST connector.

In the following sections of this chapter the challenges of battery management and system regulation decisions will be discussed in further detail. For the sake of ease of testing and development, a number of batteries using a standard JST connector were used for evaluation with the remainder of the components described in this chapter.

\subsection{Battery Charging and Management}

As referenced in the previous section of this chapter, the decision of precise battery chemistry was left, in part, to the power management and regulation portions of the subsystem design process. This section will better describe the motivation for on-board battery management in the context of rechargeable and nonrechargeable chemistries.

\subsubsection{Battery Management ASIC}

One key consideration in the design of any system making use of rechargeable battery chemistry is the importance of precise control of current into and out of the cell over the course of the charging process. Often, without customized charging circuitry designed for the specific cell topology, battery charge lifetime is significantly reduced within tens to hundreds of recharge cycles. Previous TEMPO platforms have all

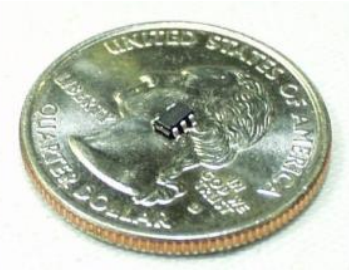
made use of a common 300mAh lithium polymer coin cell, and thus this work strongly considered the use of rechargeable lithium chemistry. Throughout the process of reviewing the previous platform's hardware, it was discovered that the ultra-low footprint MAX1555 LiPo charger ASIC used in the previous TEMPO nodes, could also be used to charge LiIon cells. Thus, the battery charging and management decision was simple. The ultra-small footprint and low passive count of the MAX1555 allowed for the device to be included in the hardware layout. If a rechargeable lithium-based chemistry is being using, this IC manages recharging the cell from the included USB connector. It sources a single,

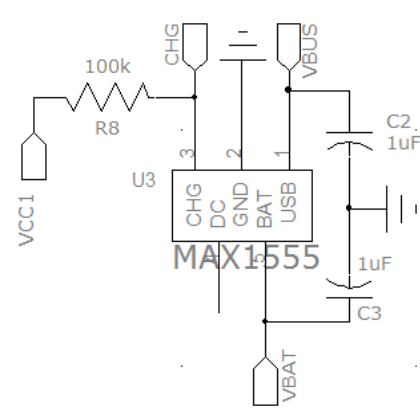

Figure 28: MAX1555 LowPassive Count Charger Circuit charge indicator pin to the MCU for the purpose of determining when the device is actively charging. When a rechargeable battery chemistry is not to be used, these components simply become Do Not 
Populates (DNPs) and there is no recharge functionality present on-board which might potentially damage a non-rechargeable cell.

\subsubsection{Reverse Voltage Protection}

In addition to charge management another consideration key to protecting the system from significant damage due to battery failure or mis-installation is reverse voltage protection. Most commonly, system regulators are not protected against reverse voltage, and as a result, when the battery is installed in reverse the regulator is the first point of failure. A relatively simple circuit trick, introduced to the group by a previous INERTIA team member, is adopted from the previous platform to protect against reverse voltage situations. This circuit is provided for reference in Figure 29. Notice that the back-EMF protection diode is used forward-biased in this control scheme, pulling up the far side of the PMOS transistor and turning the transistor on for full current conduction in the channel. This circuit is useful as it provides a lowprofile reverse voltage protection solution that can be easily shorted out on the board at population time if the feature is not desired, for example if a soldered battery connection is being used.

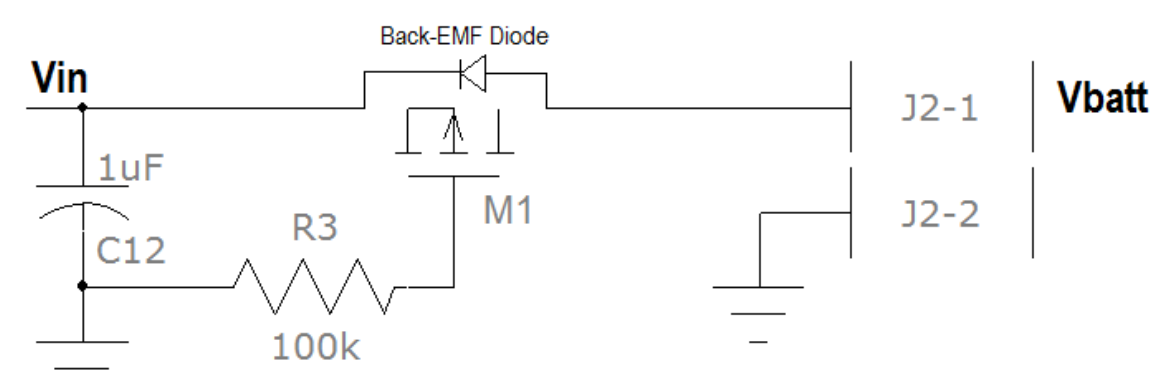

Figure 29: Low-Profile Reverse Voltage Protection Circuit

\subsubsection{Battery Management Layout}

With the MAX1555 circuit, low-profile JST connector, and reverse voltage protection PFET, the overall battery management area was kept to about $10 \%$ of the targeted 1 "x 1 " form factor. This was deemed acceptable, considering the incredibly low complexity of interfacing the hardware and the near autonomous protection of the system against reverse voltage and overcharging damage to the cell.

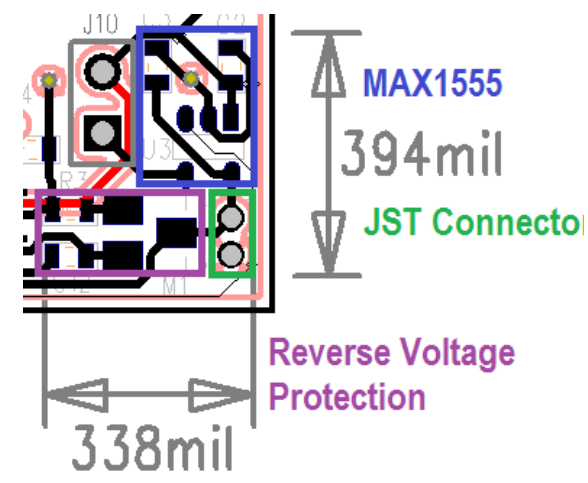

Figure 30: Battery Management Circuit Area 
In addition to providing the benefit of a minimal hardware overhead, the battery management portion of the board is considered completely optional. That is, if non-rechargeable, non-reversible batteries are used in a system, all of the components in this section, with the possible exception of the JST connector itself, can be simply left out or shorted to maintain the power path, as is the case with the reverse voltage protection PFET.

\subsection{Supply Regulation}

One of the most important considerations that eventually drove a majority of the interest in lithium-based chemistries for the TEMPO 4 platform was that of supply regulation. As opposed to battery management, the regulator is a key part of any embedded electronic system as it provides stable, reliable DC voltage to various on or potentially off-board components for operation.

Low-power applications pose an interesting challenge for power system designers interested in energyefficient regulation. The traditional evaluation of regulator topology for low power systems promotes use of switching regulators as they, on average provide more efficient regulation and need not have an efficiency strongly correlated with the input-to-output voltage differential. However, there are some situations in which low input-to-output differentials, small current draws, and stringent area constraints significantly reduce the efficacy of switching regulators. This work attempts to propose one such application and demonstrate the improved efficiency of LDO linear regulators in this regime.

\subsubsection{Linear Regulators}

A linear regulator is a voltage regulation device that uses an analog feedback loop to lock its output voltage to either an internal or external reference voltage regardless of input voltage, provided it exceeds the sum of the dropout voltage and the desired output voltage of the device.

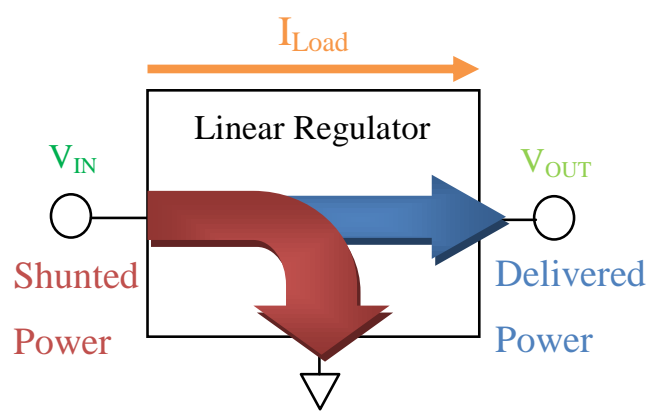

Figure 31: Linear Regulator Power Flow 
A linear regulator functions by essentially "shunting" any voltage above the desired output voltage, often provided by an internal band gap referenc, to ground. The easiest way to think about the power dissipated in a linear regulator is by considering an ohmic model of the device. Since all current delivered to the load, in this case our system, is passed through the regulator, and the voltage drop across the regulator can be calculated as the difference of the input and output voltages, and we can find the power consumed in the regulator and delivered to the load using an Ohmic model as follows.

$$
\begin{gathered}
P_{\text {reg }}=I_{\text {load }}\left(V_{\text {source }}-V_{\text {load }}\right)=I_{\text {out }}\left(V_{\text {in }}-V_{\text {out }}\right) \\
P_{\text {load }}=I_{\text {load }} V_{\text {load }}=I_{\text {out }} V_{\text {out }}
\end{gathered}
$$

Thus we can express the maximum efficiency, or the best-case ratio of power delivered to the load to power drawn by the device, of any linear regulator as follows:

$$
\frac{P_{\text {load }}}{P_{\text {tot }}}=\frac{P_{\text {load }}}{P_{\text {reg }}+P_{\text {load }}}=\frac{I_{\text {out }} V_{\text {out }}}{I_{\text {out }}\left(V_{\text {in }}-V_{\text {out }}\right)+I_{\text {out }} V_{\text {out }}}=\frac{V_{\text {out }}}{V_{\text {in }}-V_{\text {out }}+V_{\text {out }}}=\frac{V_{\text {out }}}{V_{\text {in }}}
$$

For this reason, linear regulators are often used in applications where the output voltage, which must be lower than the input voltage, is a significant fraction of said applied input voltage. For example, when lithium polymer battery chemistries $(\sim 3.6 \mathrm{~V}$ cells) are regulated down to standard $3.3 \mathrm{~V}$ system operating voltage efficiencies above $80-90 \%$ are achievable in linear regulator topologies.

In addition to the benefit of predictable efficiency regardless of load current, linear regulators require relatively few off-chip passives, usually just two capacitors used for input and output decoupling, and low-power parts, where less power is dissipated on-die, have reduced their pin-count and package size significantly in recent years. As a result of this smaller package size and low off-chip passive count, linear regulators have the added bonus of being able to produce multiple, electrically isolated and regulated outputs, at various voltages if desired, without consuming significant amounts of board area.

\subsubsection{Switching Regulators}

Switching regulator topologies are more varied, and generally speaking, less restrictive than their linear counterparts. The fundamental concept of switched-mode regulation is the use of a switch-control feedback circuit, rather than a continuous-control comparator circuit, to converge on the desired output voltage. As a result of this switched-mode operation the input-output relationship of a switching regulator is much more difficult to model. In "boost" based topologies, it is possible for the DC output voltage to exceed the input voltage, and thus these regulators perform poorly when stepping down voltages. Instead, "buck" or DC-DC converters are commonly used for step-down applications. Generally speaking, 
switching regulator efficiency is often a function of specificity of design, with far greater diversity in available topologies and commercial products than linear alternatives.

For years, switching regulators have been dominant in energyconstrained applications as their non-linear characteristics allow for battery voltage boosting, low heat-dissipation, and incredibly high efficiencies when stepping large pack voltages, in the 12$24 \mathrm{~V}$ range, down to commercially complaint levels (i.e. 1.8, 3.3, 5 , and $12 \mathrm{~V})$. However, as devices start to support operation at lower and lower voltages (1.2-1.6V) to save energy, and Lithium and Zinc-based chemistries further reduce pack voltages $(\sim 3 \mathrm{~V})$, the need for large voltage drop across on-board regulators
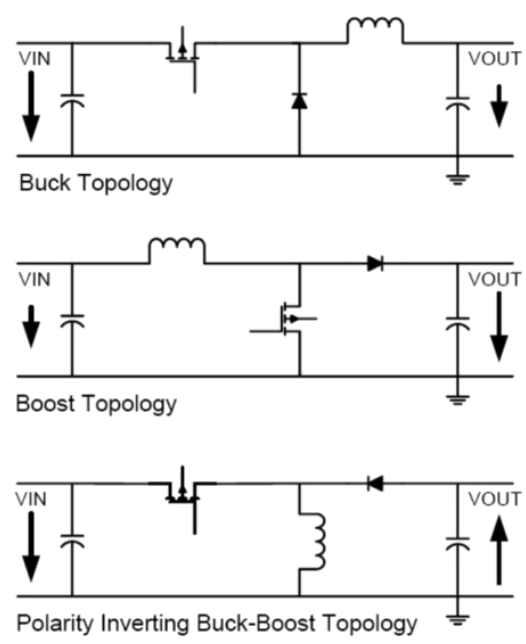

Figure 32: Switching Regulator Topologies [35] decreases, as does the traditional market-share of many of these switched-mode topologies.

The most commonly cited draw-back of switching regulators is the need for additional off-chip passives not required by other classes of regulators. Since one of the concepts fundamental to switched-mode regulation is the idea of storing energy in an inductor or capacitor during the period where the switching circuit is off (input and output voltage electrically isolated) the efficiency of switched-mode regulators is often dictated in a large part by the quality factor of inductors, or size of capacitors used to store energy and regulate line voltages. Traditionally speaking, these metrics are strongly affiliated with the physical size of these components, and thus more efficient operation also means a larger area consumed for supply regulation.

The design and layout of a switched-mode, boost converter was previously performed by a fellow INERTIA team member to source $6.3 \mathrm{~V}$ to the DC charging apparatus in the TEMPO 3.2 charger. In this case a non-linear part was required as the system output voltage exceeded that of the input, $5 \mathrm{~V}$ from the charger's USB connection. In Figure 33 the result of this layout process is included for reference. It can be easily seen that of the $1.5 \times 1.5$ " of the charger board, nearly half of the layout is consumed by the boost converter and affiliated passives. 


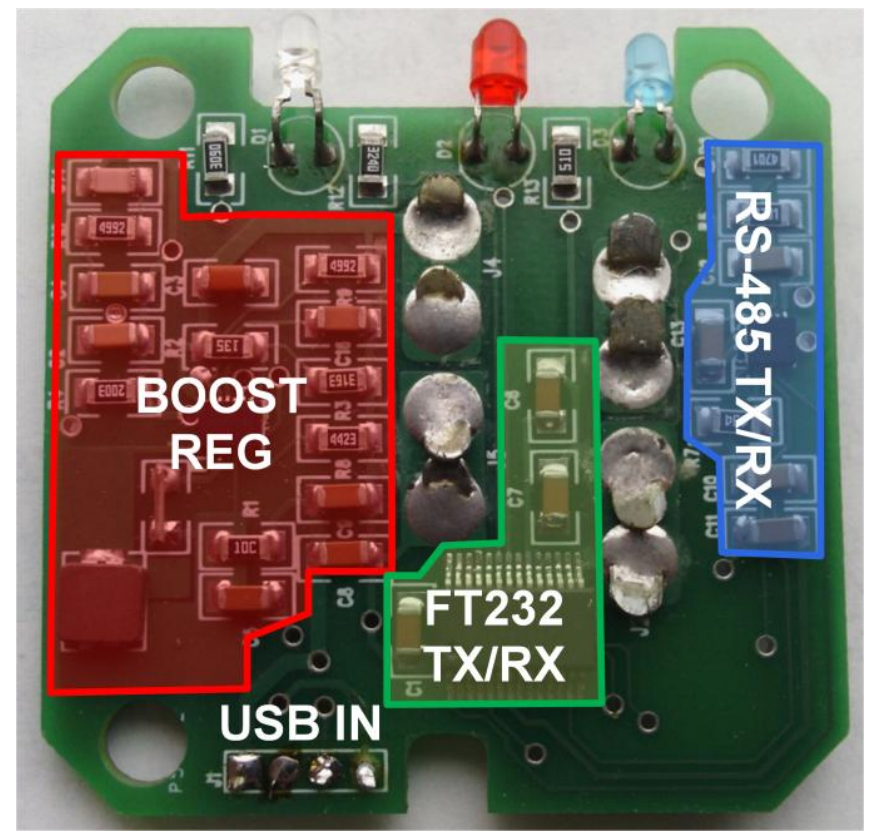

Figure 33: TEMPO 3.2 Charger with Boost Regulation, FT232, and RS-485 translation IC

A less-often cited draw-back of the switched-mode regulator is its efficiency degradation for small forward currents. Since many of these regulators operate by rapidly switching their outputs constantly, often at high frequencies produced on-chip, as the total load current drops the percentage of the input power-budget spent on switching increases, resulting in poorer efficiencies at low forward currents. As a result of this phenomenon, most switching regulators have an optimally efficient load current that is significantly larger than that targeted by this work, in the range of micro-to-milliamps.

\subsubsection{State of the Art Comparison and Regulator Decision}

As a result of the relatively high efficiencies of Low Drop-Out (LDO) linear regulators with small forward voltages, and the relatively low load currents $(\mu-\mathrm{mA})$ required for this application an LDO linear regulator topology was selected for use in the TEMPO 4 platform. Specifically, the AP7312 dual-output LDO linear regulator from Diodes Incorporated was chosen, as it can provides two electrically-isolated, controllable, 150mA outputs, one of which is used for on-board voltage regulation, the other for providing supply to the 16-pin generic header to be discussed in a later session.

Once an LDO linear regulator topology was selected, its output voltage was specified. While many components used in the TEMPO 4 system work can operate over a wide variety of operating voltage, the standard compliant 3.3V level was selected for system operation. This was in part motivated by a desire to support higher frequency operation of the MCU and also in part to allow regulation or division down to lower standard voltage levels. Once this $3.3 \mathrm{~V}$ operating point was selected, it automatically implied that 
lithium chemistries would be a good fit for this application, as they produce nominal voltages between 3.6 and $4 \mathrm{~V}$, resulting in efficiencies as high as $92 \%$ for a $3.3 \mathrm{~V}$ output. This also aligned with the battery management conclusion to include on-board LiPo/LiIon charging circuitry, finalizing the design decision.

\subsection{Battery Management and Regulation Summary and Conclusions}

The final solution arrived at for battery supply, management, and regulation is intended to optimize flexibility for future developers with varied system power constraints. By providing a standard JST connector, any battery or battery pack, provided it produces at least $3.5 \mathrm{~V}$ nominal output voltage, may be used with the system. Battery charging is accomplished via the on-board USB connector and a MAX1555 Lithium-based battery management IC. If a nonrechargeable chemistry is to be used with the platform this

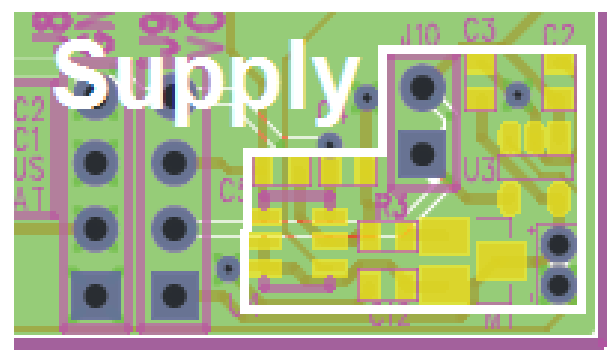

Figure 34: Testboard Supply and Regulation Layout (with 100 mil header at left for reference) battery management chip is simply not populated in the final design. Last, but not least, the use of a $3.3 \mathrm{~V}$ dual output AP7312 LDO linear regulator from Diodes Inc. implies that while larger pack voltages are tolerable for the system, they do imply poorer regulation efficiencies. With a LiPo or LiIon cell connected to the TEMPO 4 platform, regulator efficiency near $80 \%$ across the typical operating current range is expected. A full circuit schematic for system supply and regulation is provided in Figure 35 below along with the system test board layout in Figure 34.
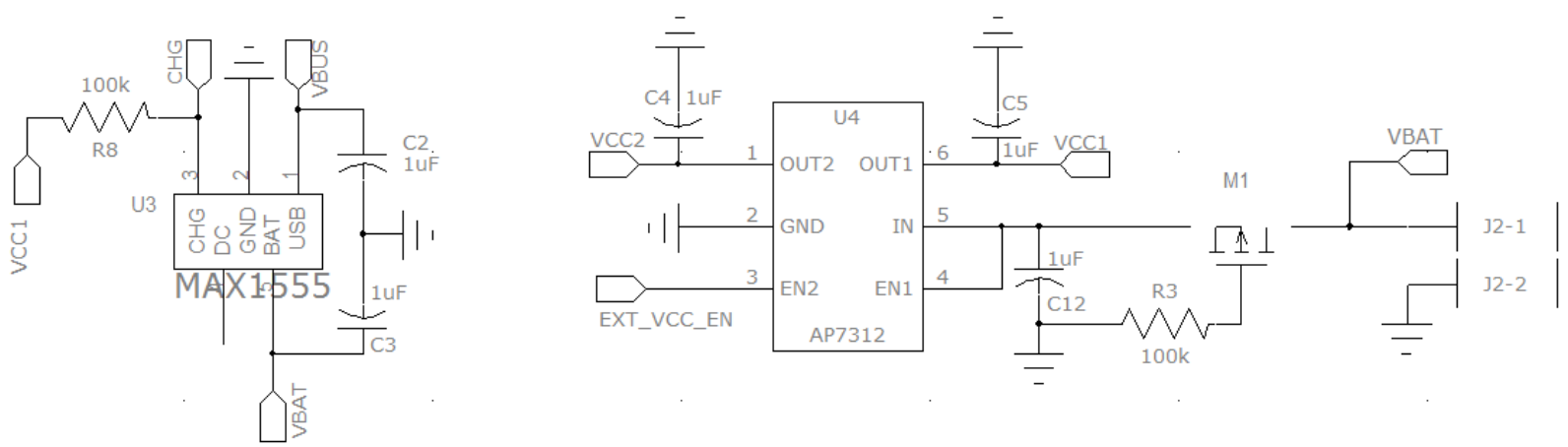

Figure 35: TEMPO 4 Battery Supply and Regulation Circuitry

The co-design concepts introduced in this section focus on the trade offs of form-factor, lifetime, and flexibility. In regard to battery chemistry, when exploiting the volume, mass, and energy-density benefits of Lithium-based chemistries, it is important for application designers to consider the power constraints they imply on system operation, including attempting to better level system power network loading in 
order to reduce peak input currents as much as possible. During the discussion of battery management, the high impact of ASIC products on the design space is discussed, along with the firmware benefits of using these tightly-integrated products in hardware design, including autonomous operation and easily interpretable charge indicator signaling. Last, but not least, during the supply and regulation section of this chapter the importance of consideration of nominal cell voltage and expected increase in the use of Lithium battery chemistries is used to motivate selection of a non-traditional linear regulator over a more complex switched-mode device, for the purpose of increased efficiency and reduced hardware footprint. 


\section{Chapter 5}

\section{Control, and Programming and}

\section{Interfaces}

The principle design challenge in determining the limits of end-point flexibility and ease-of-use for the TEMPO 4 platform was that of programming and interfacing the node. A number of tentative hardwarefirmware solutions to the generalized problems of controlling node operating and sampling were proposed, but ultimately no one-size fits all conclusion could be drawn. For this reason, rather than focus on enabling the maximum possible extent of system operation under a singular unified operating model, this section focuses on achieving reasonable goals for the programming, control, and interfacing of the TEMPO 4 system based on iterative development of a series of rigorously test firmware libraries.

\subsection{System Controller Selection}

Arguably the most important challenge in any embedded system design problem is that of MCU selection, and for the purpose of this chapter's organization it is the challenge that will be addressed first. In this section the challenge of controller selection is discussed in three parts, selection of controller topology, discussion of commercial-off-the-shelf (COTS) parts that fill into the selected topology, and ultimately selection and development on top of an individual part based on co-design trade-off analysis.

\subsubsection{A Brief Survey of Controller Topologies}

To begin the controller section process a brief qualitative market survey including a variety of topologies of controller units was conducted, followed by extensive discussion of the MCU selection decision with a variety of INERTIA team members and affiliated technical collaborators. Several key categories of contenders appeared, each with various advantages and disadvantages. Three primary candidate topologies are summarized below.

\section{Hardware Definable Controller Solutions: FPGAs and CPLDS}

This set of controllers consists of those which implement entirely, or nearly entirely, programmable logicbased solutions to coordinate system operation. Common realizations of programmable logic solutions are those of Field Programmable Gate Arrays (FPGAs) and Complex Programmable Logic Devices (CPLDs), 
both of which make use of a set of widely programmable processing blocks and precisely controllable interconnect arrays in order to allow fully user-defined hardware to be implemented in the device.

The primary advantages of implementing hardware-defined control are those of flexibility and robustness. Since multiple control paths can be processed in parallel, with any level of redundancy and isolation from the remainder of system control events, robustness is increased. In addition the vast hardware-flexibility of these platforms allows for users to create almost any structure, from a simple state machine to a full implementation of a commercially available core [36] in the programmable logic fabric. This provides for the broadest possible scope of developer-defined system control to be captured by these controller topologies.

Unfortunately, the lowered ease-of-interfacing for developers, higher power consumption during operation, and increased form-factor that comes with many of these devices often does not justify their use in ULP applications. More recently, some companies such as Xilinx, Altera, and Lattice have all sought to change that, bringing programmable logic into the low-power commercial market [37]. However, for now higher cost and legacy support for serial-execution processors has limited the success of these solutions.

\section{Hardware Reconfigurable Controller Solutions: Hard-core FPGAs and PSoC}

More recently a number of hardened silicon design firms have ventured into the programmable logic design space. Earliest examples of this work include simple PLA and PLD technologies. Today a number of tightly integrated reconfigurable hardware solutions are available on the market, from powerful FPGAs with hardened processor cores [37] to the Programmable System-on-Chip (PSoC) from Cypress [38].

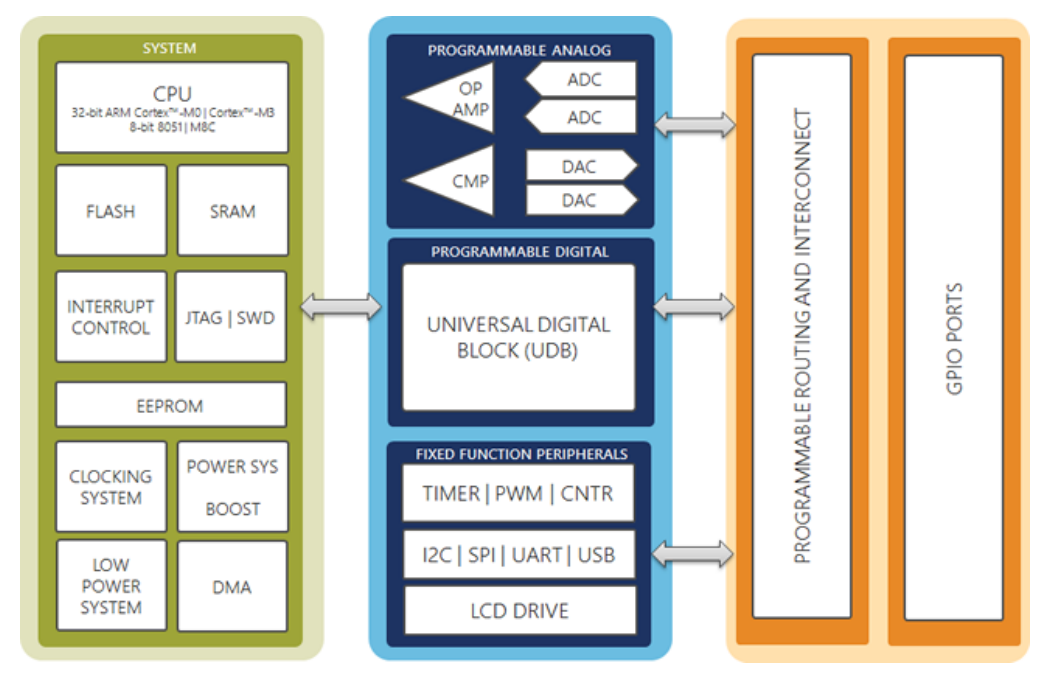

Figure 36: Cypress Programmable SoC System Topology [38] 
The previously mentioned Cypress PSoC series was considered more closely for its ability to enable a new-found level of flexible system operation, while providing a comfortable C-based programming interface to developers. Two series of the PSoC, one with an 8-bit 8051 core and another with a 32-bit ARM M0 were both contenders. Unfortunately though the development software is free and fairly intuitive to use, it was found to be quite difficult to implement novel hardware-defined functionality in the FPGA fabric without use of pre-developed Cypress libraries. Unfortunately as a result of this the PSoC failed to realize a number of the potential power and reliability benefits it's topology was selected for. Though the PSoC chips are promising candidates for use in future iterations of the TEMPO platform, or hardware add-ons, their relatively immature, non-standard topology and lack of widespread adoption resulted in them not being considered in the final pool of candidate host-controllers.

\section{Flexible Hardware Controller Solutions: MCUS}

This family is entitled "flexible" hardware solutions to imply that most if not all commercially available, fixed hardware implementations of MCUs allow some degree of flexibility. Typically pins can always be configured as either digital inputs or outputs, as well as to special functions that may be affiliated with each pin, or a given set of pins referred to as a port. In addition it is typical for peripheral modules to contain a number of configuration registers, designed to meet the needs of as many end-point applications as possible with a single hardware block in silicon. In addition many MCU designers are looking increasingly towards widespread use of crossbar switches and port-mapping controller solutions to allow for even greater flexibility in output pin assignment. Currently SI Labs leads the way in this effort with nearly fully-flexible, mixed-signal crossbar functionality on all their 8 and 32 bit MCUs [39].

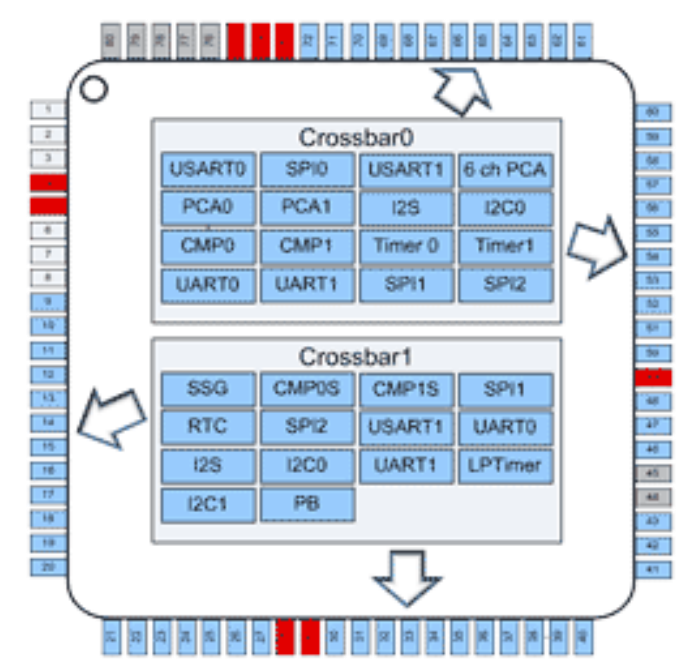

Figure 37: SI Lab Split I/O Crossbar Switch Design [39] 
Since the power, ease-of-interfacing, and form-factor considerations implied by the previous two subsections demonstrated them to be infeasible immediate solutions for the TEMPO platform host controller, this level of hardware flexibility was deemed sufficient for the desired operation. Though this decision to pursue flexible hardware solutions restricts the problem of controller selection to that of commercially available MCUs, it will be shown that significant effort is still required to determine which devices will best suite both system designers' and developers' needs.

\subsubsection{Operating Constraints and MCU Selection}

In order to constrain the results of a market survey and ensuing discussion to those that would suit the needs of medical and technical collaborators alike, a brief set of operating constraints was synthesized for the purpose of limiting the scope of market evaluation.

Based on feedback from on-node processing efforts in previous TEMPO systems, which had a maximum system clock rate of $8 \mathrm{MHz}$, a greater maximum operating frequency, of at least $16 \mathrm{MHz}$, is desired for the TEMPO 4 system controller. It was also specified that the MCU did not consume more than 10mA at $16 \mathrm{MHz}$ operation, a rather pessimistic bound for device operation. Working backwards to the industrystandard metric, this implies an active current of less than $625 \mathrm{uA} / \mathrm{MHz}$ at $16 \mathrm{MHz}$.

In addition, the ability of TEMPO to maintain accurate wall-clock timing and produce high accuracy, regularly spaced sample windows was also prioritized. For this reason, the TEMPO 4 node also calls for an MCU implementing at least 2 system timers along with a real-time clock (RTC) module for maintaining wall-clock time during device operation. It was also specified that these timers be sourced from an off-chip, high-precision crystal oscillator for the sake of mainting the quality of system and sample timing offered up by previous platforms. A second peripheral space consideration for the MCU is that of being able to interface a wide array of analog and digital products with easy-to-use hardwareimplemented peripherals. For the purpose of this work at least 2 ADC channels along with peripheral support for several common serial standards was considered as the bare-minimum for device interfacing.

Last but not least the issue of code and data memory size was addressed briefly in the establishment of a lower bound for reasonably flexible and full-featured system operation. The previous TEMPO platform makes use of an MSP430 device from the F1XXX series with 10kB of SRAM and a rather large flashbased program memory available to the developer. For the purpose of this work an SRAM size of greater than $5 \mathrm{kB}$ and program memory of at least $16 \mathrm{kB}$ were considered as minimums. All of the considerations described above are summarized in Table 10 below. 


\begin{tabular}{|l|l|}
\hline \multicolumn{1}{|c|}{ Feature } & \multicolumn{1}{c|}{ Specification } \\
\hline Frequency & $>16 \mathrm{MHz}$ \\
\hline Max Active Current (@ 16MHz) & $625 \mathrm{uA} / \mathrm{MHz}$ \\
\hline On-chip Timing Peripherals & 2 Timers, RTC, Crystal-sourced \\
\hline Analog Inputs & $>2$ channels \\
\hline Supported Serial Interfaces & UART, SPI, I2C \\
\hline Minimum SRAM (data mem) size & $5 \mathrm{kB}$ \\
\hline Minimum Flash (code space) size & $16 \mathrm{kB}$ \\
\hline
\end{tabular}

Table 10: TEMPO 4 MCU Operating Constraint Summary

There are a large number of companies currently developing fixed-form MCU solutions for the ULP design space that fit the specification above including, but not limited to: the xMEGA from Atmel, PIC XLP series from Microchip, EFM32 from SI Labs, and MSP430 and Wolverine from TI. With so many options to choose from, it can be difficult to determine a precise candidate platform that is best suited for all possible end-point applications. As previously mentioned, instead of using a lengthy research period and background study to attempt to solve the problem of what is the"most flexible" fixed-implementation MCU, a brief state-of-the-art survey followed by copious review and discussion was used to determine various commercial devices' suitability for use.

In a round-about way this discussion returned to using an MSP430 platform in the next TEMPO platform because it meant developing hardware around familiar microcontroller supply, clocking, and decoupling circuitry, and that firmware code created for the previous TEMPO platform could possibly be ported to run on the newer TEMPO system. In addition the availability of a free, albeit code-size limited, Eclipsebased Integrated Development Environment (IDE) [40] and a number of tutorials and operating systems for the MSP430 platform make it an ideal candidate for flexible operation in the firmware context. In addition, the conclusions discussed in the programming portion of this chapter also support the decision to use the MSP430 platform, as it offers up a low pin-count, easy-to-interface, and full-featured debugging protocol that can be accessed using a common, low-cost, commercially available platform.

\subsubsection{MSP430 Family and Device Selection and Prototyping}

With the decision to use an MSP430 series microcontroller [40] finalized the question then came to which particular device to use. Though this may seem like a straight-forward challenge, when a product search for MCUs with the desired metrics was conducted on TI's website it returned over 30 different devices from 11 different part families. For this reason, an undergraduate student assisted with profiling the devices across a wide variety of metrics, producing the result that, of the selected set of controllers, the lowest footprint and pin count parts were all in the F534x family. A table of results from this preliminary MSP430 product survey is included below. 


\begin{tabular}{|c|c|c|c|c|c|c|c|}
\hline Part Number & $\begin{array}{c}\text { Frequency } \\
(\mathrm{MHz})\end{array}$ & $\begin{array}{c}\text { Flash } \\
\text { (KB) }\end{array}$ & $\begin{array}{l}\text { SRAM } \\
\text { (B) }\end{array}$ & GPIO & $\begin{array}{l}\text { Timers } \\
\text { 16-bit }\end{array}$ & $\begin{array}{c}\text { ADC } \\
\text { Channels }\end{array}$ & Package \\
\hline \multicolumn{8}{|c|}{ F241x Family } \\
\hline MSP430F2410 & 16 & 56 & 4096 & 48 & 2 & 8 & 64VQFN, 64LQFP \\
\hline MSP430F2416 & 16 & 92 & 4096 & 48 & 2 & 8 & 64LQFP, 80LQFP \\
\hline MSP430F2417 & 16 & 92 & 8192 & 48 & 2 & 8 & 64LQFP, 80LQFP \\
\hline MSP430F2418 & 16 & 116 & 8192 & 48 & 2 & 8 & 64LQFP, 80LQFP \\
\hline MSP430F2619 & 16 & 120 & 4096 & 48 & 2 & 8 & 64LQFP, 80LQFP \\
\hline \multicolumn{8}{|c|}{ F247x Family } \\
\hline MSP430F247 & 16 & 32 & 4096 & 48 & 2 & 8 & 64LQFP, 64VQFN \\
\hline \multicolumn{8}{|c|}{ F248x Family } \\
\hline MSP430F248 & 16 & 48 & 4096 & 48 & 2 & 8 & 64LQFP, 64VQFN \\
\hline \multicolumn{8}{|c|}{ F249x Family } \\
\hline MSP430F249 & 16 & 60 & 2048 & 48 & 2 & 8 & 64LQFP, 64VQFN \\
\hline \multicolumn{8}{|c|}{ F261x Family } \\
\hline MSP430F2616 & 16 & 92 & 4096 & 48 & 2 & 8 & 64LQFP, 80LQFP \\
\hline MSP430F2617 & 16 & 92 & 8192 & 48 & 2 & 8 & 64LQFP, 80LQFP \\
\hline MSP430F2618 & 16 & 116 & 8192 & 48 & 2 & 8 & 64LQFP, 80LQFP \\
\hline MSP430F2619 & 16 & 120 & 4096 & 48 & 2 & 8 & 64LQFP, 80LQFP \\
\hline \multicolumn{8}{|c|}{ F532x Family } \\
\hline MSP430F5324 & 25 & 64 & 6144 & 48 & 4 & 16 & 64VQFN, 80BGA \\
\hline MSP430F5325 & 25 & 64 & 6144 & 63 & 4 & 16 & 80LQFP \\
\hline MSP430F5326 & 25 & 96 & 8192 & 48 & 4 & 16 & 64VQFN, 80BGA \\
\hline MSP430F5327 & 25 & 96 & 8192 & 63 & 4 & 16 & 80LQFP \\
\hline MSP430F5328 & 25 & 128 & 10240 & 48 & 4 & 16 & 64VQFN, 80BGA \\
\hline MSP430F5329 & 25 & 128 & 10240 & 63 & 4 & 16 & 80LQFP \\
\hline \multicolumn{8}{|c|}{ F534x Family } \\
\hline MSP430F5340 & 25 & 64 & 6144 & 31 & 4 & 9 & 48VQFN \\
\hline MSP430F5341 & 25 & 96 & 8192 & 31 & 4 & 9 & 48VQFN \\
\hline MSP430F5342 & 25 & 128 & 10240 & 31 & 4 & 9 & 48VQFN \\
\hline \multicolumn{8}{|c|}{ F541xA Familiy } \\
\hline MSP430F5418A & 25 & 128 & 16384 & 67 & 3 & 16 & 80LQFP \\
\hline \multicolumn{8}{|c|}{ F543xA Family } \\
\hline MSP430F5435A & 25 & 192 & 16384 & 67 & 3 & 16 & 80LQFP \\
\hline MSP430F5437A & 25 & 256 & 16384 & 67 & 3 & 16 & 80LQFP \\
\hline \multicolumn{8}{|c|}{ F552x Family } \\
\hline MSP430F5521 & 25 & 32 & 6144 & 63 & 4 & 16 & 80LQFP \\
\hline MSP430F5522 & 25 & 32 & 8192 & 47 & 4 & 16 & 64VQFN, 80BGA \\
\hline MSP430F5524 & 25 & 64 & 4096 & 47 & 4 & 16 & 64VQFN, 80BGA \\
\hline MSP430F5525 & 25 & 64 & 4096 & 63 & 4 & 16 & 80LQFP \\
\hline MSP430F5526 & 25 & 96 & 6144 & 47 & 4 & 16 & 64VQFN, 80BGA \\
\hline MSP430F5527 & 25 & 96 & 6144 & 63 & 4 & 16 & 80LQFP \\
\hline MSP430F5528 & 25 & 128 & 8192 & 47 & 4 & 16 & 64VQFN, 80BGA \\
\hline MSP430F5529 & 25 & 128 & 8192 & 63 & 4 & 16 & 80LQFP \\
\hline \multicolumn{8}{|c|}{ F663x Family } \\
\hline MSP430F6638 & 20 & 256 & 16384 & 74 & 4 & 16 & 100LQFP, 113BGA \\
\hline
\end{tabular}


Once the MSP430F534x family of MCUs was selected, individual device selection was only a function of available memory size. For the sake of making a large amount of system SRAM available to future designers, and maintaining memory-size compatibility with previous TEMPO platforms, the MSP430F5342, with 10kB of on-chip SRAM was selected for use in this work. It is worth noting, that if less memory and lower affiliated cost is desired, all chips in this family are pin-compatible, implying an easy bill of materials swap for future platform producers.

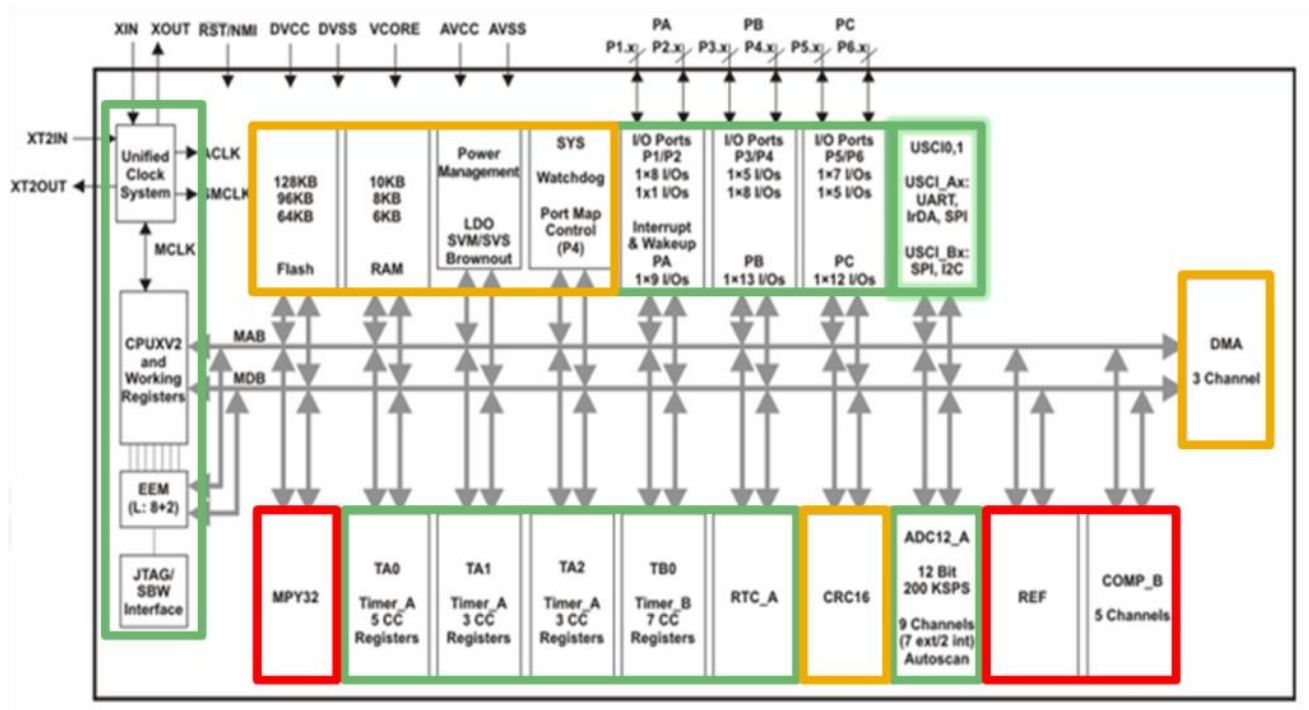

Figure 38: MSP430F5342 System Diagram [41]

The MSP430F5342 is quite a capable chip for its size, featuring programmable on-chip oscillators and Frequency Lock Loop (FLL), a 16-bit MSP430 core, 4 Universal Serial Communication Interfaces (USCIs), 2 timers, an RTC with calendar mode, Spy Bi-Wire (SBW) programming, a port mapping controller, and a number of other useful peripherals all in a 48-pin VQFN package. More about the use of these features of this MSP430 device will be discussed throughout the reaminder of this section and document.

Once the MSP430F5342 was selected for evaluation, a number of hardware test benches, with varying levels of integration of the core platform, were used to verify the device's operating specification and better examine system performance. Images of several of the selected hardware test-bed platforms are included in Figure 39 below. 

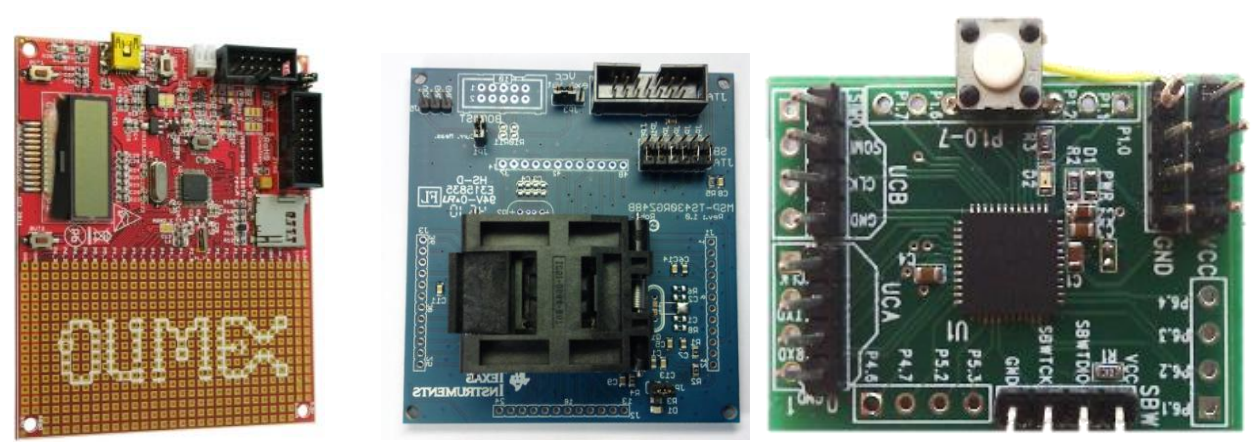

Figure 39: MSP430F5342 Development Platforms

(Left-to-right: Olimex MSP430-5510STK [42], TI MSP430F534x 48-Pin Target board[43], and custom breakout for power measurement)

Here again, it is important to remember that though this work is described in linear order of subsystem design, it was not in fact conducted in this way. The Olimex development board was used for the purpose of evaluating on-MCU USB transceiver solutions, and then later adopted as an available platform for early creation of code for configuration of common resources such the timer modules, RTC, clock control via the FLL, and some portions of the communications library. Unfortunately, the architecture of the 5510 chip's serial communication interface differed slightly enough that though this platform did have an onboard MMC port it was not able to be used for early porting and development of the TEMPO 3.2 MMC libraries.

The socket-based platform from TI was used for the most extensive portion of early system prototyping and code development. Though it may be difficult to see in the image above, this platform uses a solderless socket to connect directly to the QFN pads of an MSP430 48-pin QFN package. It then implements an easy-to-use on-board 14-pin JTAG connector along with simple single-inline pin header breakouts for all 48 pins of the device. By wire wrapping or connecting these pin headers to other development boards with ribbon cables, a number of early system prototypes were able to be tested on the bench without the need for custom PCB development.

Last, but not least, at the same time as the TEMPO 4 system test board and MPU6000 breakout board, referred to later in this work, were created and produced, an additional custom, low-footprint MSP430 breakout board was also created. This board's function was two-fold. First, by producing a minimal pin count interface with little-to-no extra on-board circuitry this platform created a viable means for the precise measurement of system core voltage and current consumption at runtime. Second, the smaller and simpler population job of this low component count board resulted in a dramatically reduced turn-around time, and an ability to test the MSP430 supply, programming, and control circuitry independent of the remainder of the components included in the more complex TEMPO 4 system test board. 


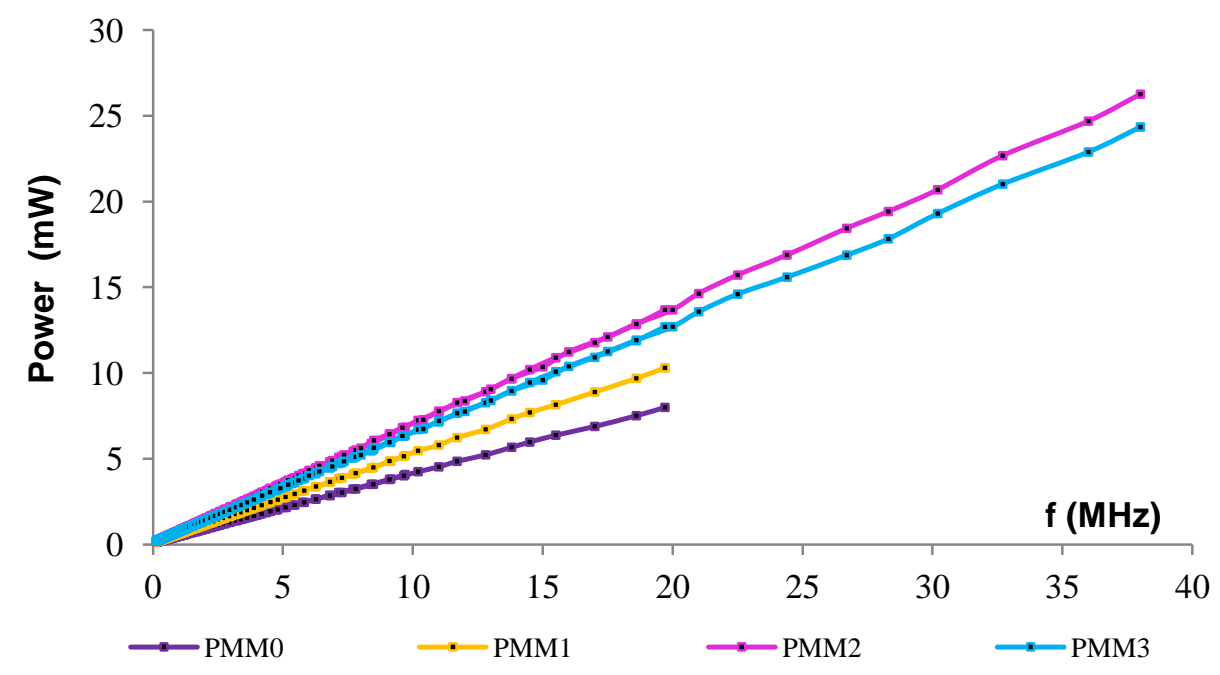

Figure 40: MSP430F5342 Power vs Frequency Plot w/ FLL Controlled Operation

Using the custom MSP430 breakout board described above the power versus frequency profile of the MSP430 was obtained for each of the 4 core operating voltages the device is capable of operating at, set by a programmable on-chip LDO regulator at runtime. The results of this power profiling are seen in Figure 40 .

This on-chip core voltage regulator is by default configured to provide the minimum input voltage to the core, resulting in the lowest system power and also the smallest range of valid frequencies for system operation. However, if desired, application coders can raise the core voltage level, allowing for higher processing frequencies, seen in Table 12, at the cost of quadratic increase in system power and energy. In addition to being a useful feature for additional power consumption reduction or expansion in suitable applications, this on-chip regulator, which is separated from a second integrated regulator used for I/O voltages, makes this particular MSP430 platform an interesting candidate for Dynamic Voltage Scaling (DVS) based solutions.

\begin{tabular}{|c|c|c|c|}
\hline Mode & Min. $\mathbf{V}_{\mathbf{C C}}$ & V $_{\text {Core }}$ & Max. $\mathbf{f}_{\text {op }}$ \\
\hline PMM0 & $1.8 \mathrm{~V}$ & $1.4 \mathrm{~V}$ & $8 \mathrm{MHz}$ \\
\hline PMM1 & $2.0 \mathrm{~V}$ & $1.6 \mathrm{~V}$ & $12 \mathrm{MHz}$ \\
\hline PMM2 & $2.2 \mathrm{~V}$ & $1.8 \mathrm{~V}$ & $20 \mathrm{MHz}$ \\
\hline PMM3 & $2.4 \mathrm{~V}$ & $1.9 \mathrm{~V}$ & $25 \mathrm{MHz}$ \\
\hline
\end{tabular}

Table 12: MSP430F5342 Power Management Mode and Core Operating Condition Definitions

An independent study conducted outside of the scope of this work more carefully examined the feasibility of implementing DVS control in the MSP430 using a break-even time model that compares the device's DVS energy consumption to that of an aggressive wake-sleep control configuration. Unfortunately, despite the interesting opportunity for investigation, it was deemed that this MSP430 platform's low sleep power, and relatively high active currently implied a break-even runtime near 3 seconds. This means the 
MCU would need to process continuously for 3 seconds in order to amortize away the extra cost of not sleeping for the slack time produced by running at a higher core frequnecy. Since this amount of uninterrupted runtime is not considered typical for the targeted set of TEMPO 4 applications and this analysis did not consider the added code overheads affiliated with USCI reconfiguration during frequency-scaled operation, it was considered unlikely for a DVS solution to yield practical benefit over standard sleep-wake, or duty-cycled, operation in the TEMPO 4 use-case.

\subsection{MSP430 Programming}

Although the previous section concludes with the selection of a single MCU-device for use in the TEMPO 4 platform it was not conducted agnostically of the programming portion of this subsystem design. Instead, each candidate platform in the previous section of this chapter was also evaluated for ease-of-programmability and availability of programming interfaces. The top candidates, including the Cypress PSoC and MSP430 were then evaluated for their ease of development through the use of development hardware and freeware tool chains.

The conclusion to use the MSP430 from Texas Instruments is supported by a number if intermittent conclusions arrived at throughout the course of prototyping and programming firmware for use in many of the early test benches created as part of this work. This section will focus on three primary areas of consideration for programming and development interfaces.

\section{Physical overheads}

\section{Cost, availability and ease-of-interfacing}

\section{Backend software support and debugging considerations}

More information about each of these areas is provided in the affiliated sub-sections below.

\subsubsection{Physical Considerations Overheads}

One challenge to be considered explicitly for the TEMPO 4 platform was that of physical overheads affiliated with system programming interfaces. While at first glance this may not appear to be a vital consideration, it is in fact, a significant challenge for many modern open development platforms. For example, the TEMPO 3 systems all made use of a custom-external programming board, as seen in Figure 41, adapting a standard 14-pin JTAG connector to a high-density, lower-area connection for the sake of on-board device programming. This was largely due to the fact that integrating the 14-pin JTAG connector directly into the platform would have resulted in significant form-factor increase. 

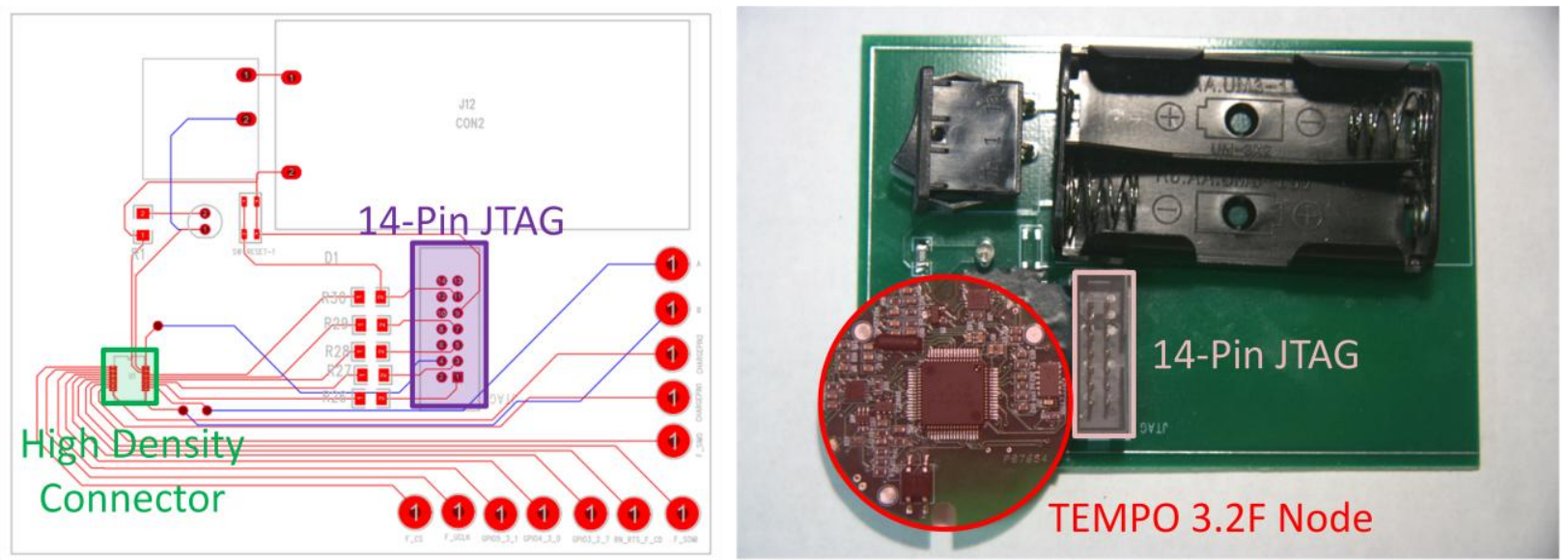

Figure 41: TEMPO 3.2 Custom Programming Adapter

Unfortunately, this small, high-density connector made both the TEMPO and interface boards difficult to assemble, and also required relatively little lateral force to damage beyond repair once installed. Thus early in the design process it was decided that the TEMPO 4 platform would not make use of any specialized high-density connectors. The figure below provides images of the TEMPO 3.1 and 3.2 highdensity programming connectors, as well as an example of the damage that can be caused do the connector by physical stress.
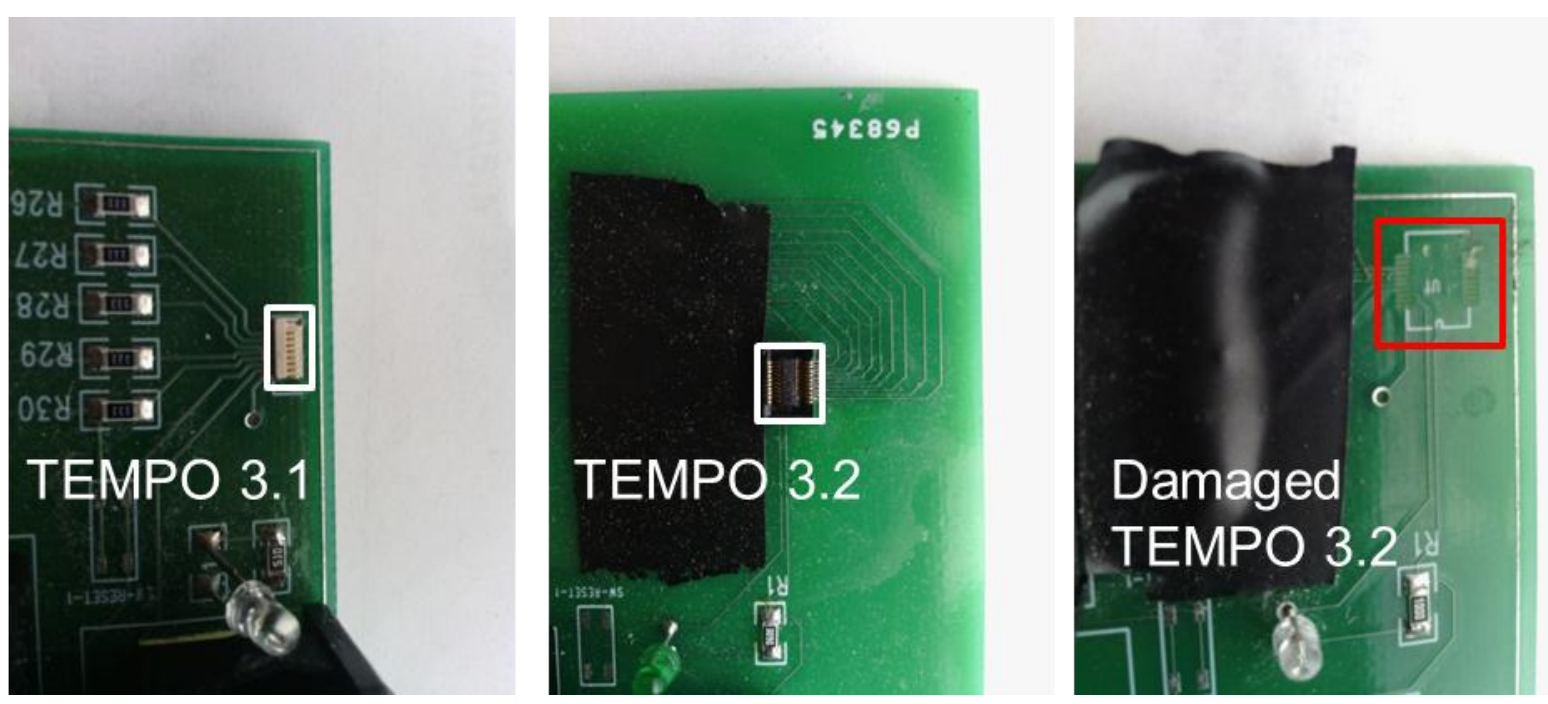

Figure 42: TEMPO 3.1 and 3.2 High-density Programming Connectors with Example of Damage to 3.2Connector Though a number of standard exist for programming microcontrollers, some of the most common programming strategies include either fuller-featured 10-16 pin JTAG connections or smaller, serialized programming connectors that may offer up less debug functionality in a lower-pin count, or more common interface. Texas Instruments stands out in their efforts to optimize these lower pin-count interfaces without reducing full debug functionality. Currently, of the 14-pins present in the JTAG 
connector TI only requires the use of four in full JTAG interfacing. In addition the company has introduced the Spy Bi-Wire (SBW) serialized 2-wire programming interface for even lower footprint programming overhead [44].

For the purpose of this work the SBW debug interface was selected for interfacing the MSP430 controller. This added that constraint that the selected MCU must have

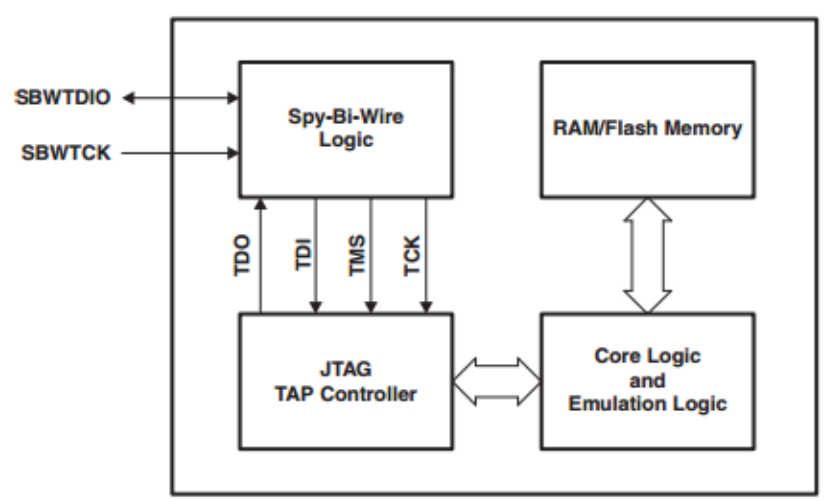

Figure 43: Texas Instruments Spy Bi-Wire Operational Concept [44] the interface available for use, as only TI's newer MSPs have the SBW interface implemented on them, and that little to no additional programming hardware should be required on the board. The integration of this 2-wire interface into the 16-pin development header is discussed during the interfaces section of this chapter.

\subsubsection{Cost, Availability, and Ease-of-use}

This section addresses a lesser-considered challenge to using many commercially available MCU devices; that of sourcing programming hardware and using the desired programming interface on a day-to-day basis. Though this may not seem like a critical constraint in the modern design space, often times hardware programming and debug interfaces for more complex platforms can host hundreds or thousands of dollars, and require expensive back-end software for the purpose of interfacing and debugging the hardware during the programming process.

Fortunately a clever, low-cost, and high-availability solution for SBW programming of the MSP430 was arrived at just prior to the beginning of this design work. The TI Launchpad platform, a \$10 development board from TI, including a value-line MSP microcontroller, two pushbuttons, two LEDs, and a USB programming and communication port, represents a significant step forward in getting beginners and hobbyist markets involved in programming the MSP430. 


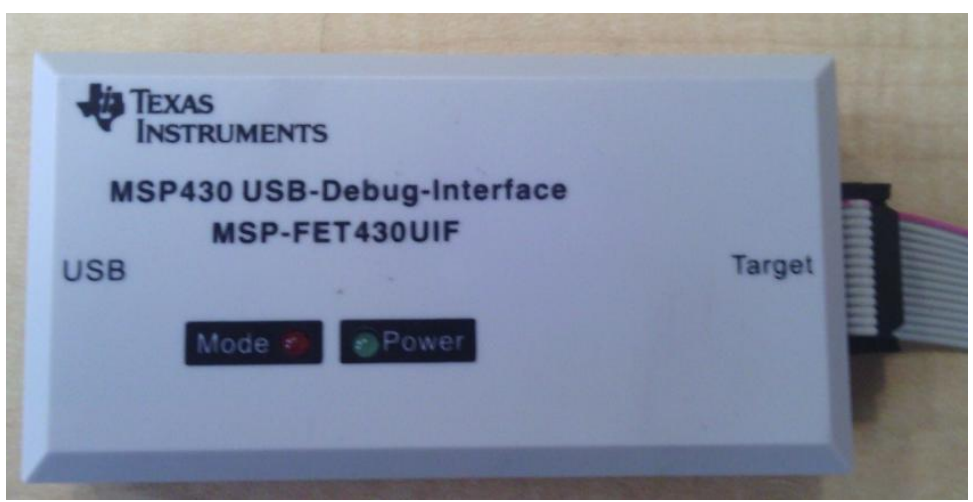

Figure 44: MSP430FET USB-based Programmer

In order to be able to program this device TI needed to implement their proprietary SBW programming method in a self-contained way, as traditional MSP USB to JTAG programmers cost between $\$ 50$ and $\$ 150$ [45]. In order to accomplish this, a chain of TI-based ICs is used to accomplish USB to SBW conversion in the top half of the Launchpad platform. Even more impressive, is the ability of this USB port to be simultaneously used as both a debug interface and plug-and-play serial communication port during on-board testing and evaluation. However, these serial communication capabilities will not be required for use in this work.

\subsubsection{Final Device Programming Solution}

By using the emulation to evaluation jumper pool on the LaunchPad and connecting to the TEST and RESET signals from the emulation side of the device, without connections to evaluation side made, the platform can be used as a USB debugger for any MSP430 system using a SBW interface. In fact, if desired, the emulation portion of the board could be cut away entirely, leaving only the SBW connections and a ground available for the user. The figure below demonstrates this use of the Launchpad as a widelyavailable, stand-alone USB programmer for less than $\$ 10$.

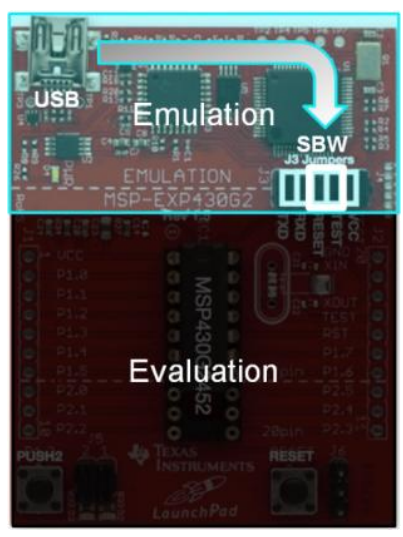

Figure 45: MSP430 Launchpad Platform as a SBW Programmer with Emulation and Evaluation Portions Labeled [46] 


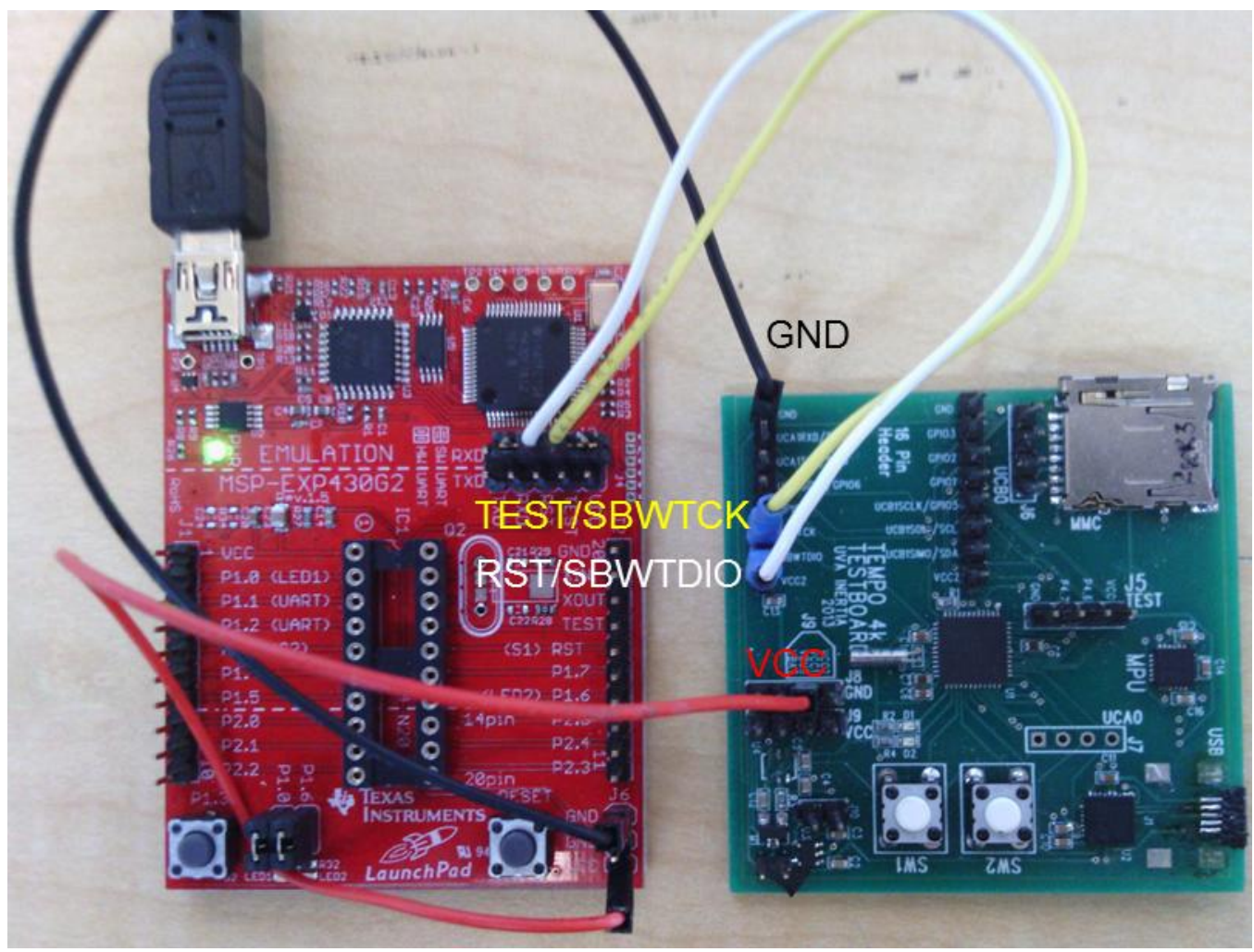

Figure 46: Launchpad Debugger Connected to the TEMPO 4 Test Board using SBW debugging interface

\subsection{System Interfaces}

One of the primary challenges addressed by this work is that of interfacing a wide variety of sensing and reporting modalities, both through analog and digital signal capture amd communication. If there is one strong suite of the TEMPO 4 platform relative to its market competitors it is that of easy-to-use serial digital interfaces and the code libraries that supports them. This section discusses both the standard and the custom interfaces available to users and developers of the TEMPO 4 platform including the 16-pin generic development header that will serve as the motivating feature for the next chapter of this document, and the on-board USB interface. The issues of wireless data reporting and MMC storage are not addressed in this chapter, as they will be dealt with at length in the coming chapter.

\subsubsection{Serial Digital Interfacing}

The first and primary challenge addressed in the development of the TEMPO 4 platform interfaces is that of digital interfacing for a variety of common serial standards. For this reason, many of the most significant co-design concepts found in this work rely upon the iterative development of a rigorously tested interfacing scheme for a number of serial standards. To begin with a brief qualitative survey of available sensor, as well as storage and radio, serial interfaces revealed three primary contenders for 
commonly implemented protocols: the Universal Asynchronous Transceiver (UART), Serial Peripheral Interface (SPI), and Inter-Integrate Circuit (I2C). Each of these three standards will is discussed below in the context of implied system-wide constraints, co-design principles and impacts on the desired metrics.

\section{UART Interface}

The UART interface has two primary advantages which are most likely related. The UART is, outwardly a rather simple interface. Two separate RX and TX lines, along with a common ground return path, communicate data in full-duplex with no sharing of the clock, and thus no strongly delineated master or slave. Possibly as a result of this outward simplicity and historical significance, the UART interface is quite common. The RS-232 standard, used by most personal computers and referred to as the "serial" port, is a UART connection that operates with a $10-24 \mathrm{~V}$ nominal swing that can be easily down-converted for use with more common embedded signaling voltages. For this reason a number of sensor and system manufacturers provide UART interfaces to their platforms and modules. Though typically UART operates at baud rates below $1 \mathrm{Mbps}$, some commercial devices will communicate with UART baud rates up to and exceeding 3-10Mbps

At first glance the UART seem to be a near ideal interface for low-power serial communication. By sending data asynchronously, or whenever it is ready, and not transmitting a clock along with this data both power and energy savings are reaped. However, taking a deeper look into the operation of a UART interface can help answer the question as to why asynchronous data recovery is often a more energy hungry system-level operation than it may appear.

When a UART transmitter prepares to launch a packet of data, typically a single byte in most cases, it must first turn on its own baud rate generation. In most modern systems this is rather easily accomplished as the UART module has its baud sourced from an internal system oscillator being used to source other hardware, such as the core or timers, and thus the clock has already stabilized. Before the transmitter places the first bit of data onto the line, it first signals a start bit by pulling the line low to indicate to the receiver data is about to be transferred. This start bit may last one or two baud periods depending on what the system designer specifies. The start bit is followed by 8 baud periods, during which each bit of data is transferred serially. At the end of this window, some devices may choose to also send a stop bit indicating the end of transmission. This is commonly used for detection of packet framing errors, as the transfer window begins with a ' 0 ' and ends with a ' 1 ', it makes sense that packet overrun framing errors can be detected based on these criteria. 


\begin{tabular}{|c|c|c|c|c|c|c|c|c|c|c|}
\hline Bit & 1 & 2 & 3 & 4 & 5 & 6 & 7 & 8 & 9 & 10 \\
\hline Symbol & Start bit & \multicolumn{5}{|c|}{ 5-8 data bits } & & Stop bit(s) \\
\hline Value & 0 & D0 & D1 & D2 & D3 & D4 & D5 & D6 & D7 & 1 \\
\hline
\end{tabular}

Table 13: 8-Bit UART Bit Sequencing

In order for the receiver to capture the data being sent by the master it must respond to the start bit and begin locking its input delay to the rising edge of this bit. If the baud is slightly mistimed by the receiver this does not mean the bits will necessarily be misread by the module. Only if baud is severely enough mistimed that a bit is mis-latched (i.e. wraps around a half /whole baud window, depending on the latching edge) does baud timing become an issue. It is important to note that when discussing baud mistiming accumulating until it "warps" around a baud window, it is not implied that this occurs within a single cycle. Rather, the accumulated baud offset, which is linearly proportional to bits per word, is what cannot grow greater than some fraction of a baud period. This is illustrated in Figure 47 below.

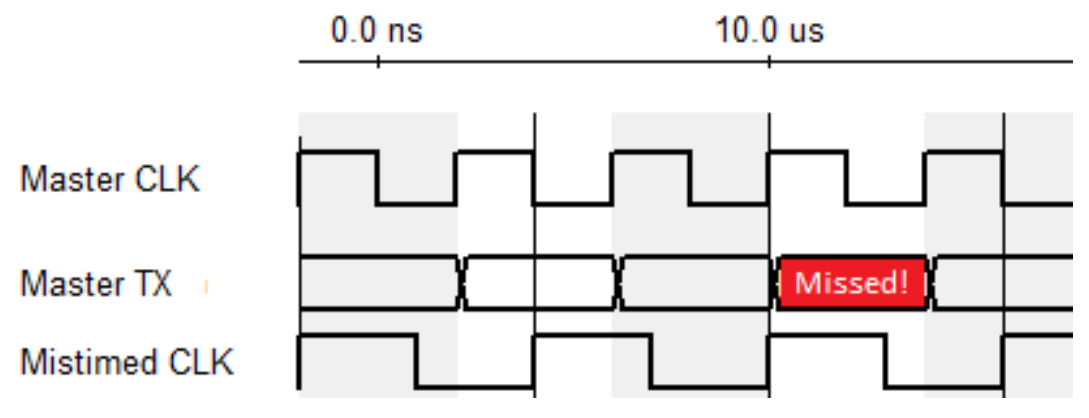

Figure 47: Baud Rate Slip in UART Communication

The maximum toleratble baud timing slip $(\Delta \mathrm{T})$ can be expressed as function of the number of bits in a word $(\mathrm{N})$ and a fraction $(\alpha)$ of the baud period $(\mathrm{T})$ as shown in the inequality below.

$$
N \Delta T \leq \alpha T_{\text {baud }} \Rightarrow \Delta T \leq \frac{a T_{\text {baud }}}{N} \Leftrightarrow f_{\text {timing }} \geq \frac{N f_{\text {baud }}}{\alpha} \text { for } a \leq 1
$$

Thus on-chip measurement and locking of the baud rate must occur within 1 baud period, and also lock within $\Delta \mathrm{T}$ of the true rate, where $\Delta \mathrm{T}$ represents the maximum baud mistiming such that the accumulation of this slip over the entire word transmission does not result in a missed bit. The resulting dual statement for frequency implies that the frequency of the clock used to time/lock the incoming data for this signal must be at least $\mathrm{N}$ times as fast as the baud of the incoming message to be successfully latched by the receiver.

For this reason, most 8-bit UART modules require that the baud divisor, baud rate clock sourced from a higher rate clock divided down, be no less than 8 , the output data length of the module. This reduces the maximum throughput of these devices and also increases the receiver power, as the frequency of 
operation need be, in some cases, much higher than the desired baud rate. In cases of high throughput interfaces (>1Mbaud), this can often make UART operation the constraining factor in overall system clock rate, and in some cases (>3Mbaud) can begin to prohibit UART operation in the ULP context altogether.

In addition to the issue of clock generation and incoming data reception, UART is also burdened with the downside of being a primarily single-endpoint driven bus protocol. The addition of a number of other bus signaling pins, formalized in the RS-232 standard, can help to extend UART to multi-endpoint applications in necessary scenarios, but at the cost of additional GPIO dedicated to bus control. As a result of significant control overheads and form-factor constraints, multi-endpoint UART communications were not considered as part of this work.

Despite some of the draw backs of the UART interface it was targeted as a desired objective in this work for two primary reasons. First and foremost, the USB to UART transceiver IC used for communications with the PC demanded at least one dedicated UART. Secondly, the microcontroller platform selected for the TEMPO 4000 design has 2 on-board UART/SPI modules capable of using either protocol. As a result, while one interface is dedicated to the on-board USB connection, the other is free to be configured as either a UART or SPI by the user prior to firmware compilation-time.

The UART portion of the communications library was based in part on a previous, in-group library written by two previous students for an older MSP430 platform. It improves upon this previous work by offering user-allocated data storage and a more minimalist data-management function API while trying to maintain the interrupt-driven code architecture and data storage methodology of the old library.

\section{SPI Interface}

The SPI is the simplest of the three common serial interfaces implemented as part of this work. SPI operates using two simple shift register structures, one device, referred to as the master, sources the clock for these two registers and data is swapped from one shift register into the other on each clock edge. By allowing the programmer to read/write these registers from either device after or during communication this data flow allows for full duplex data exchange. By sharing a single clock, sourced at the full baud rate from the master to the slave, both ICs do not need to pay the price of any baud multipliers. As a matter of fact, some well designed SPI interfaces allow communication at much higher baud rates in slave mode than the internals of the chip are capable of running at. 


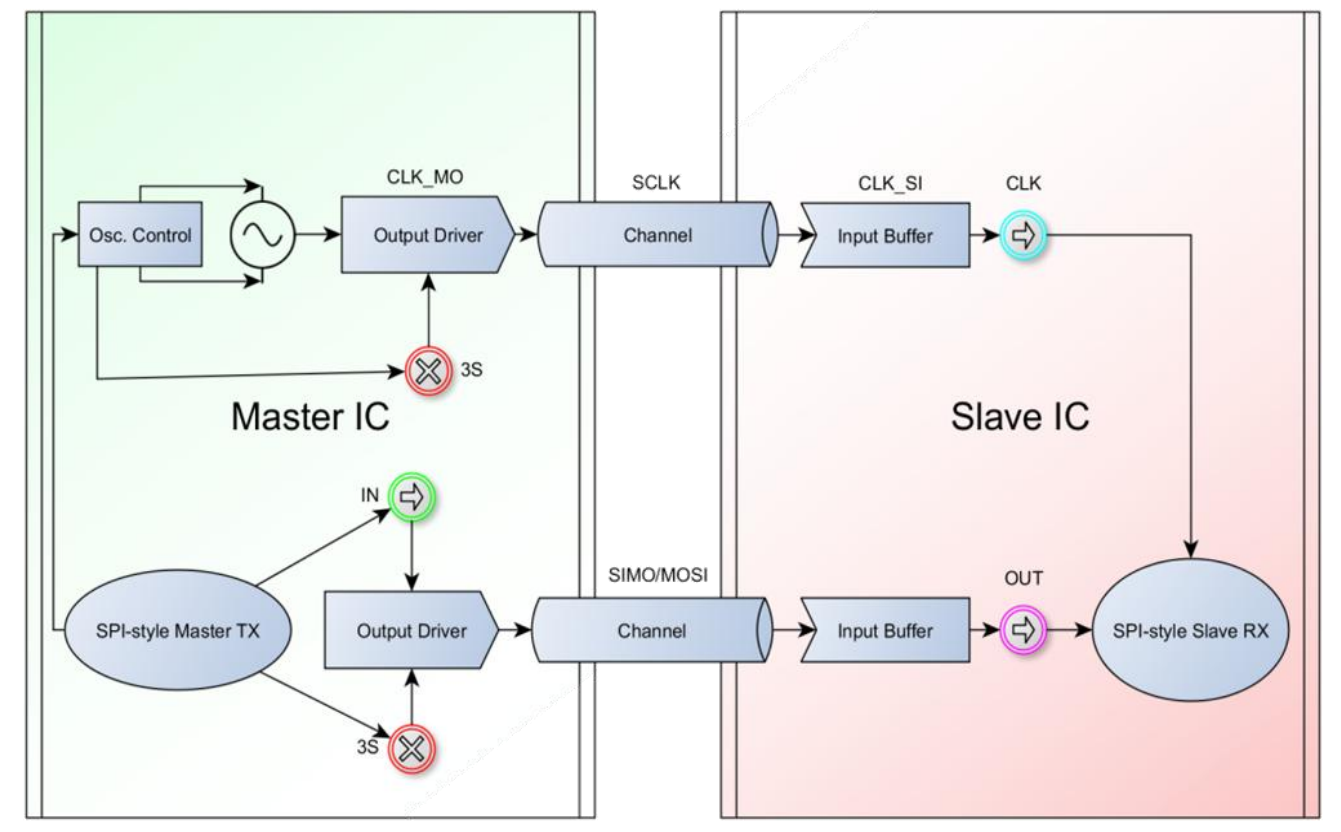

Figure 48: Typical SPI Master-Slave Topology w/ One of Two Data Connections and Clock Signal Shown

The device(s) which are sourced a clock from the master are referred to as slaves. Often an additional dedicated signal is also sourced from the master to each of the slaves. This line commonly referred to as chip-select (CS) or slave-select (SS) is used to create a parallel addressing structure for the bus, when asserted, the slave listens and communicates on the bus, otherwise it ignores all bus traffic. The result of this is a linear proportionality between SPI bus size and number of addressable devices. The core SPI communication connection is composed of 3 basic uni-directional pins along with this CS connection, outlined in Table 14 below.

\begin{tabular}{|c|l|}
\hline Pin Name & \multicolumn{1}{c|}{ Pin Function } \\
\hline SIMO & Slave In/Master Out: TX from master to RX slave \\
\hline SOMI & Slave Out/Master In: RX to master from TX slave \\
\hline SCLK & Serial Clock: Clock from master to slave \\
\hline CS & Chip Select: Optional multi-slave select line \\
\hline
\end{tabular}

Table 14: SPI Signal Description

The low hardware complexity and rudimentary operation of the SPI makes it an ideal candidate for lowpower communications. When sensors produce highly bursty data streams, such as cameras or audio modules, single or parallelized SPIs are commonly used to achieve peak data rates instantaneously while allowing for low average power consumption. An added benefit of the SPI is its lack of formal voltage specification. Though many devices operate SPI interfaces in the standard 1.2-3.3V range, there is little to nothing, except for gate delay itself, in the way of further reducing SPI voltage swing in the name of increased energy savings for lower throughput communication interfacing. 
The incredibly simplistic nature of the SPI interface makes it a good replacement for many more common serial data standards that have strong mater/slave roles. The use of SPI to communicate through the MMC standard with MicroSD cards is a good example of this ability. In addition to interfacing the on-board flash memory, a single SPI interface was also intended for communication with a 6 degree-of-freedom IMU from Invensense. Unfortunately, this part was non-responsive and ultimately warranted a move to a close I2C-based relative.

SPI is a common standard for ultra-low power reporting modalities. Devices like digital temperature sensors, barometers, accelerometers, magnetometers, etc. often employ SPI interfaces due to their flexible operating frequency and low-power/complexity for the on-chip components. For this reason, all 4 communication interfaces on the selected MSP430 MCU support SPI communication. The SPI library created as part of this work leaves chip/slave selection to the programmer, as it was found that various devices interpret this signal in various ways, making a single, hard-coded solution non-optimal.

Otherwise, the SPI library delivers simple, interrupt-driven read, write, and swap functionality for single and multi-byte transfers.

\section{I2C Interface}

Of all three serial protocols supported by the TEMPO 4000 platform inter-integrated circuit (I2C) is both the most compact, and possibly the most power inefficient. The I2C bus is an outwardly simple 2-wire bus that contains only two pins Serial Data (SDA) and Serial Clock (SCL). While the SCL pin is still sourced from the master to the slave, the SDA line is bi-directional, allowing for simple half-duplex transmission. I2C can achieve such a compact hardware footprint as it uses a more complex signaling scheme to initiate and verify communication with the slave(s).

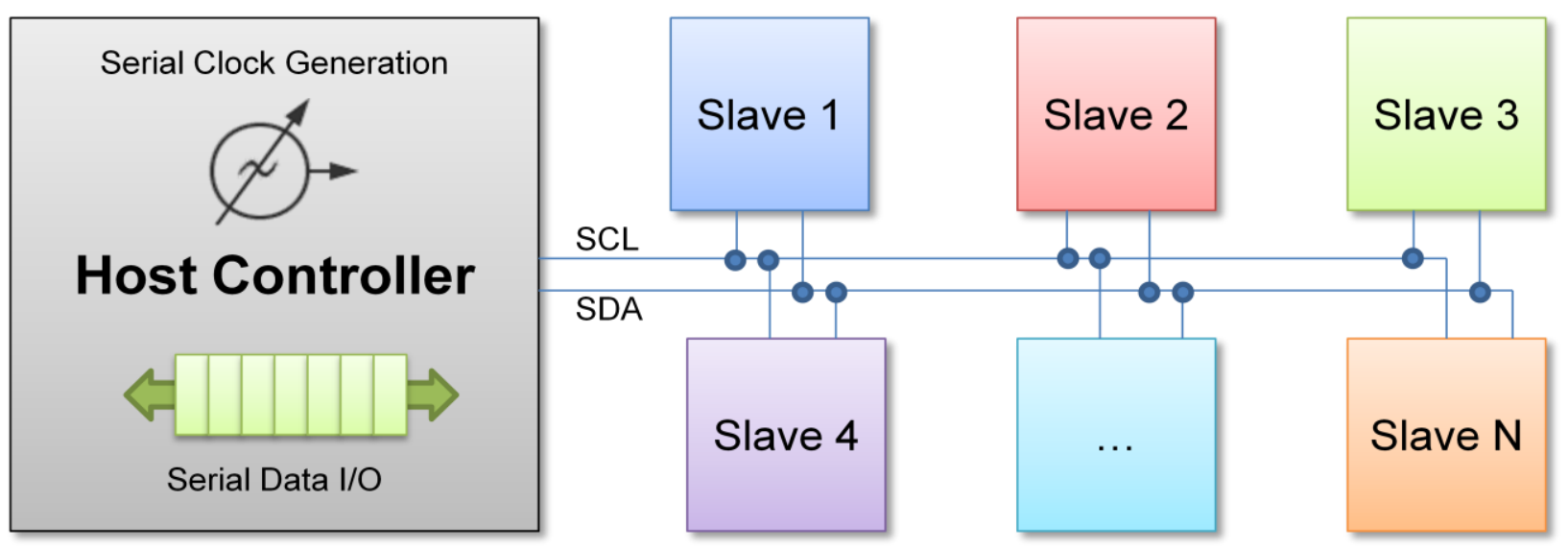

Figure 49: Example of I2C Bus Topology w/ Multiple Slave Devices 
Despite the relatively simple appearance of the $\mathrm{I} 2 \mathrm{C}$ hardware it does require some minimal additional passives for bus termination. Due to the bi-directional nature of the SDA signal in the bus, and the ability to electrically isolate the clock line from the master or the slave during reads, $\mathrm{I} 2 \mathrm{C}$ communications require one pull-up resistor be placed on each of the two I2C lines, SDA and SCL. This results in the line being pulled high whenever a device finishes transmission, signaling to the master that the bus is again available. To some extent, these pull-up resistors can be increased in value for linear power/energy savings; however, once the lumped parasitic capacitance of the line (PCB trace, pin, pad, ESD, etc.) is added to this pull-up, a low-pass filter is formed. The result is that as pull-up resistance is further increased, baud rate in the channel will have to be decreased accordingly. Outside of hardware complications, the issues involved in addressing a large, half-duplex, multi-slave environment, while time-sharing for full-duplex communications between endpoints is largely left to firmware control of the hardware unit.

For this reason, what I2C saves in hardware complexity, it often makes up for in software management. Routines required to successfully manage an I2C interface require more complex, structure-oriented APIs for higher-level programmers to easily interact with the underlying hardware. I2C data "packets" contain headers that indicate the direction of the transaction, read or write, along with address of the desired slave. As a result, multiple devices can now be connected to these two signals and share the bus provided only addressed devices respond via SDA when clocked. In addition to these packet headers, the I2C bus protocol also dictates the strict use and timing of start and stop bits, along with ACKs and NACKs designed to further improve reliability and robustness of communication.

The $\mathrm{I} 2 \mathrm{C}$ portion of the communications library also runs in an interrupt-based structure. This interrupt automates a small fimrware finite state machine (FSM) designed to complete I2C compliant communications. Unfortunately a hardware erratum, found only after the production of the first set of nodes, means that the $\mathrm{I} 2 \mathrm{C}$ interface only has timing closure up to about $50 \mathrm{kHz}$, well below the maximum communication rates of many existing $\mathrm{I} 2 \mathrm{C}$ parts.

\section{Protocol Summary and Communications Library Organization}

A table summarizing the top-level information, but not the individual design challenges, presented in each of the sub-sections above is included below. This table is not presented for the purpose of evaluating the protocols for use in the TEMPO 4 platform, as it has already been determined they will all be supported in the hardware and firmware of the system. Instead, this table is intended to demonstrate the strengths and weaknesses of each serial protocol for the sake of various possible future interface decisions. 


\begin{tabular}{|c|c|c|c|}
\cline { 2 - 4 } \multicolumn{1}{c|}{} & UART & SPI & I2C \\
\hline Max Baud & 10 's MHz & 100 's MHz & $400 \mathrm{kHz}$ \\
\hline Power & Medium & Low & High \\
\hline Multi master & No & No & Yes \\
\hline Multi slave & No & Yes & Yes \\
\hline Transfer Size & Fixed & Variable & Semi-fixed \\
\hline Pin Count & 2 (min) & $3+\mathrm{N}$ & 2 \\
\hline Availability & High & Medium & Medium \\
\hline Advantage & $\begin{array}{c}\text { Compatibility } \\
\text { Ease }\end{array}$ & $\begin{array}{c}\text { Speed } \\
\text { Power }\end{array}$ & $\begin{array}{c}\text { Flexibility } \\
\text { Size }\end{array}$ \\
\hline
\end{tabular}

Table 15: Summary of UART, SPI, and I2C Communication ( $\mathbf{N}=$ number of endpoint devices)

Based on Table 15 it can be concluded that for low-power or high throughput designs where there will not be a large number of end-point devices the SPI protocol is an intelligent choice. Meanwhile for multislave networks with low overall form-factors, I2C is the correct design decision. Last, but not least UART dominates the space of low pin-count, easy to interface, single-end point communication strategies.

The fundamental concept behind the TEMPO 4 communications library is the simplification of user interaction with low-level hardened silicon IP blocks design for various types of serial communication. Texas Instruments uses the Universal Serial Communications Interface (USCI) module as a base for all hardware serial communications. This USCI comes in two flavors; USCIA modules perform either SPI or UART communications while USCIB modules perform either SPI or I2C communications. Each MSP430 may have multiple USCIA or B modules specific to its series or family. The library addresses this by use of a custom defines file for each MSP430 device, along with conditional compilation directives designed to prune away unused routines for minimal code bloat. Currently an interface must be dedicated to a single protocol at compile time, in order to remove the possibility of off-chip cross-protocol collisions.

A communication interface is established by calling the registration function within the library. As an argument this registration function takes a communications configuration structure. This configuration structure indicates the type of module intended for use (UART, SPI, I2C), desired baud, location of a write-back pointer, and several additional control fields. However, rather than configure the module immediately, this configuration information is stored and indexed, so that the peripheral can be configured just prior to use. Since the communications library is intended to allow multiple applications to access any one physical interface, some source of program identification must be provided back to the 
developer. For simplicity, the unique index of this stored configuration information is provided, and referred to as the communication ID.

The communication library's primary sole reference to any registerd application is the communication ID. When a new user application calls the registration function with an endpoint configuration, it is returned a communication ID. This ID is analogous to a socket number. It "keeps track" of all the configuration and status information required by the library for operation. Whenever an application calls an endpoint function, such as spiA0Write(), the function takes, as one of its inputs, the application's communications ID. Using this ID the library configures the affiliated resource and then passes the message. The code is optimized to remove redundant reconfiguration, so that applications which frequently use a single interface need not pay the full reconfiguration time on each access of the device.

By implementing a simple, socket-style of communication scheme designed to minimize developer interaction with low-level hardware configuration, while still allowing skilled designers access to a majority of the underlying control mechanisms in the MSP430 hardware, this work attempts to produce a general model for communication on which to construct all other interfaces discussed as part of this work.

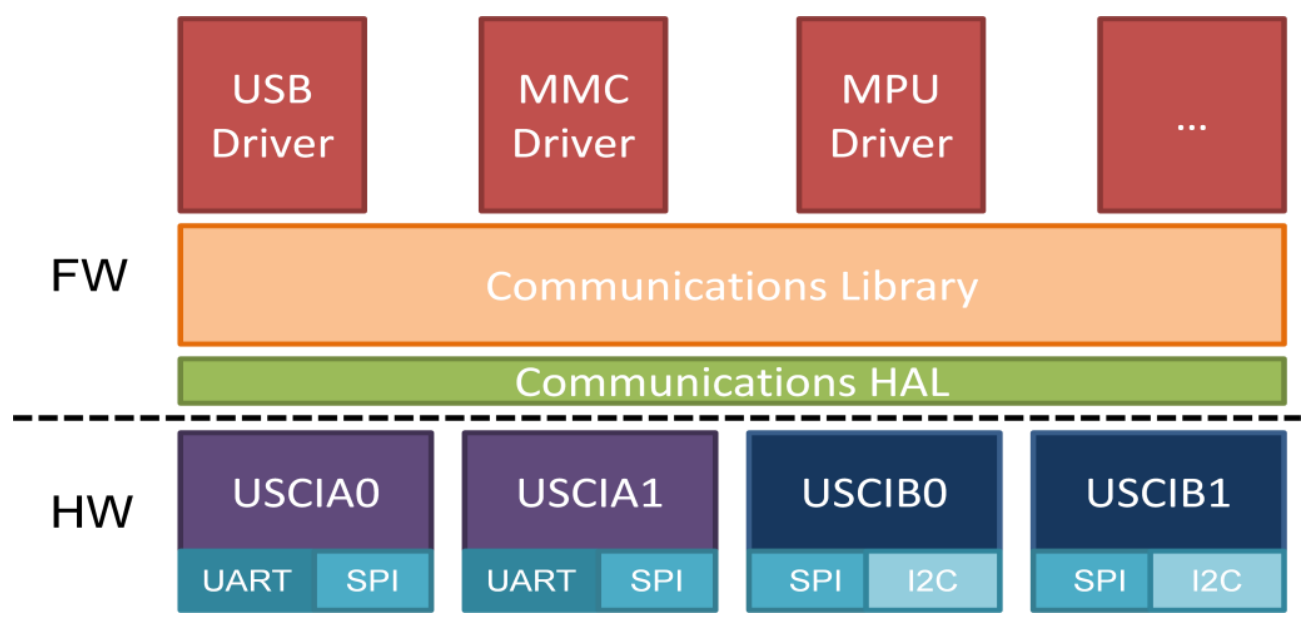

Figure 50: TEMPO 4 Communications Library Operational Hierarchy

\subsubsection{TEMPO 4 Development Header}

The primary feature of the TEMPO 4 platform's design for open development is that of the 16-pin generic header provided to the developer for interfacing the node and enabling hardware add-ons in the form of top or bottom mounted expansion boards. At this point in the design summary, this document has provided enough information to outline the pin-out of this header and describe its intended functionality. 


\section{Physical Layout and Pin Count}

The total pin count of the development header was not arrived at arbitrarily. To begin with it was established that the TEMPO 4 header should conform to standard 100 mil spacing practices, this set the platform apart from Shimmer 3, which uses custom, high-density connectors to interface user-developed hardware [5]. Next, the maximum outline of 100 mil-spaced pin headers that would allow for some minimal external routing constraints was determined. To this end, the dimensioned drawing in Figure 51 was arrived at.

This header size constraint resulted in 16-pins being fit on the board, and thus this is used for the pin-count for the remainder of this work. It is worth noting that though these pins are spaced by 100 mils vertically, they are not spaced at an even multiple of 100 mils horizontally. This is a potential negative feature as it means the board cannot be easily plugged into a standard breadboard; however, the area gains

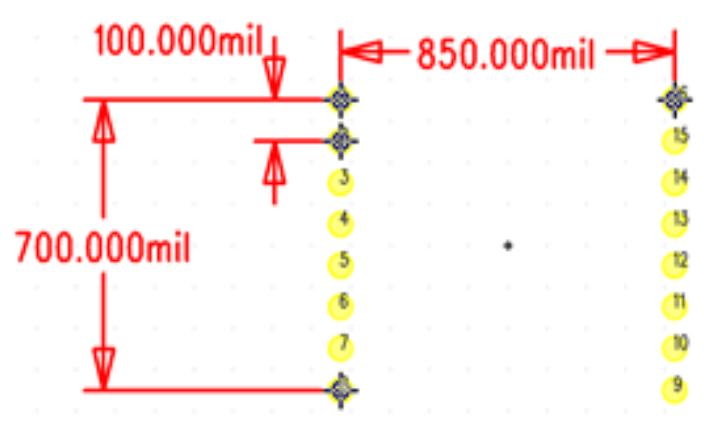

Figure 51: Dimensioned Drawing of Maximum Allowable TEMPO 4 Pin Header created by spacing these pin headers slightly farther apart were determined to out-weigh this convenience constraint. In addition, preliminary testing indicates the loose tolerances on many breadboards does in fact allow for the TEMPO 4 node to be inserted into most commercially available breadboards if desired.

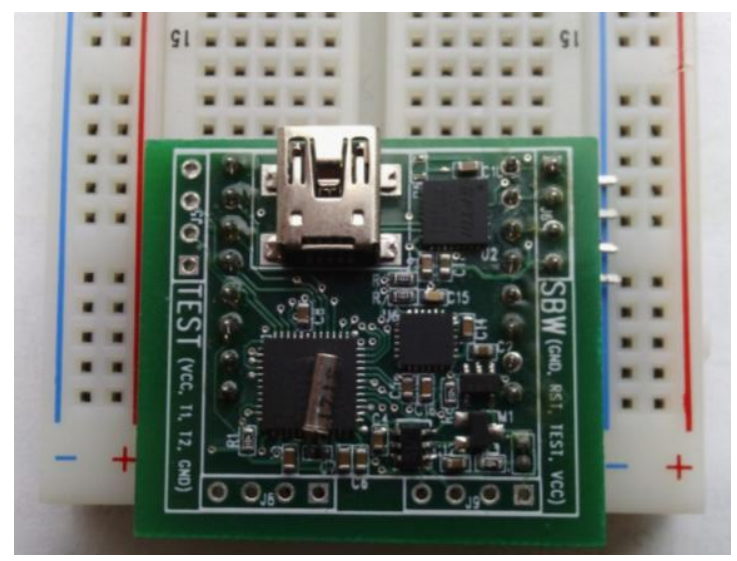

Figure 52: Final TEMPO 4 Design Demonstrating Breadboard Interfacing w/ 16-pin development header

\section{Electrical Considerations and Header Pin Out}

The TEMPO 4 development header includes two "power rails", or regulated VCC and ground, electrically isolated from on-board supply but not from each other, for powering add-on platforms. It is worth noting that applications drawing more than $150 \mathrm{~mA}$ will need separate, electrically isolated supplies. In addition to these supply pins, the header also contains the standard TEST and RESET signals that compose Texas 
Instrument's SBW programming interface. This allows for quick and easy device reprogramming through the header.
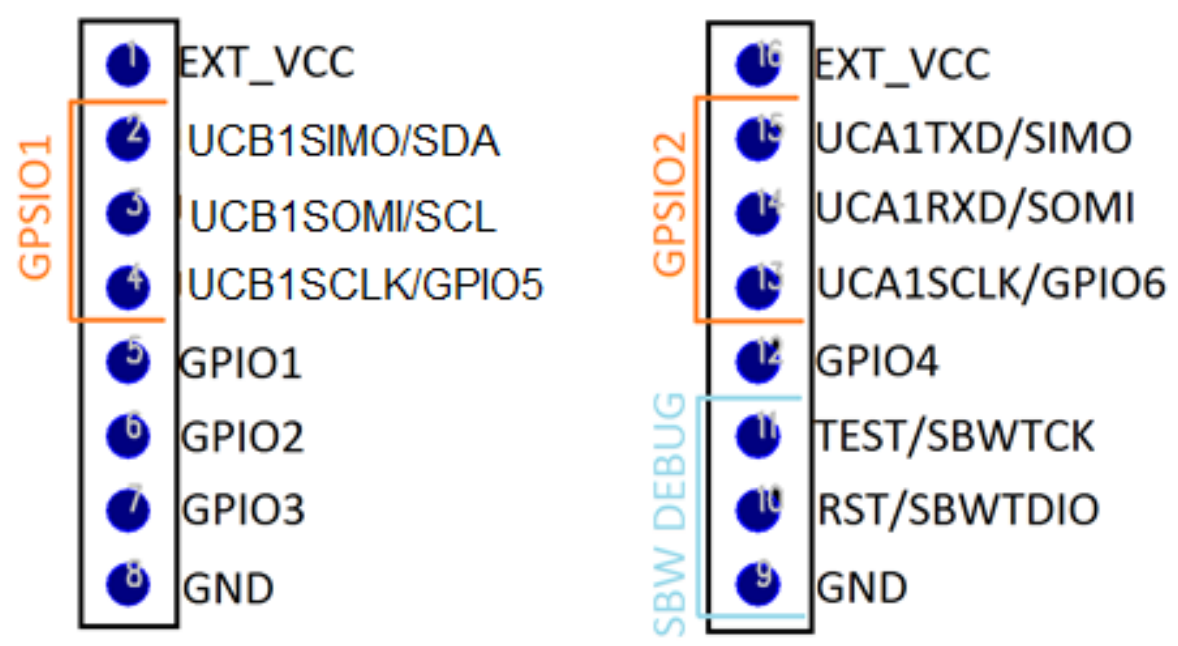

Figure 53: TEMPO 4 16-Pin Development Header Pin-out

In addition to power supply and programming, the header provides mixed-signal I/O capabilities for a variety of sensor-based applications. The first general purpose serial I/O (GPSIO1) interface is designed to be a dual-use SPI/I2C communication channel, but the use of an on-board I2C inertial motion capture unit discussed in a following chapter, if populated, means this interface should only be used for I2C communications. As previously mentioned, the microcontroller selected for this project contains an erratum that states this $\mathrm{I} 2 \mathrm{C}$ module does not have timing closure above $50 \mathrm{kBaud}$. As a result, no baud rate above 50kbps is supported on GPSIO1. The GPSIO2 interface is a fully functional general-purpose SPI/UART communication interface. UART and SPI baud rates up to $1 / 2$ of the system clock rate are supported, but it is recommended to keep these interfaces below 10MBaud for reliable operation.

The four general purpose I/O pins (GPIO1-4) support digital input and output functionality as well as analog input capture, via an integrated MCU-side 12-bit SAR analog-to-digital converter (ADC). In addition to these four dedicated GPIO inputs, any pin other than the supply and programming pins can be reprogrammed as a simple digital input/output, including the GPSIO clock lines when the interfaces are in UART or I2C (2-wire) mode.

The TEMPO 4 development header supports the communications library described as part of the previous sub-section of this chapter for easy handling of the two USCI modules included in the pin-out. For the purpose of protecting end-point devices, the ability to dynamically reconfigure between protocols, that is to switch a module from SPI to I2C operation during runtime, is prevented against using conditional 
compilation directives. However, for more skilled designers with complex system topologies in mind, there is nothing preventing this sort of code modification from being performed. Analog sampling is left to the user, with a number of code examples on ADC control and timing available from TI and other sources.

\subsubsection{USB Communication}

Though a case study included in a previous section of this work dealt with the issue of USB transceiver selection, it is worth noting the full value of this interface to the TEMPO 4 platform. Not only does USB serve as the data offload interface for the node it also provides the current and voltage for battery recharging. Thus, as mentioned, considerations regarding this USB interface were considered to be of paramount importance.

As previously concluded, the UART protocol lends itself well to the USB transceiver design space as messages are passed to and from the user asynchronously. In addition, the constant possibility of USB connection and a corresponding demand for the bus meant the USB transceiver would need to be granted its own dedicated, single-end point hardware communication interface. In the case of the FT232 transceiver selected as part of the previously mentioned case study, a UART interface demonstrated the highest ease-of-use and firmware transparency, and was thus selected as the appropriate solution.

This dedicated FT232 UART interface is managed via the previously mentioned communications library on USCIA0 of the MSP430 device. Due in part to the simplicity of the UART protocol and in part to the ease-of-interfacing of the FT232 device, the entirety of the FTDI firmware library is composed of declaration of a buffer for message storage and a set of call-through functions to pass messages in and out of the USCIA0 interface synchronously. This firmware library is intended to be used as a reference for future designers interested in developing for the USCIA1 module, which is available in the development header for future UART deployments.

\section{Charging Considerations}

The default USB port will supply up to $100 \mathrm{~mA}$ of current at $5 \mathrm{~V}$ nominal output to any inserted device. Upon device request, the USB standard supports current draws of up to $500 \mathrm{~mA}$ and thus requires a relatively reasonable level of hardware protection, which is often improved upon by commercial system designers. For this reason, USB charging and supply was seen as a reasonable direction for this work. Only the FT232 USB transceiver IC itself is powered from the bus directly, the remainder of the current consumed by the TEMPO 4 platform when plugged into a PC or outlet over USB is used to charge the 
devices battery via the MAX1555 battery charger IC. For this reason the on-board USB interface is able to serve both as the device's offload and charging interface.

\section{Offload Time Constraints}

One complaint heard about the TEMPO 3 system, which also made use of an FT232 transceiver ASIC, was that of relatively long data offload times. This resulted primarily from a $1 \mathrm{~ms}$ character delay enforced in the out-going PC-side of the offload stream for the purpose of reducing the chance of bit errors caused by unreliable node firmware-side operation during UART receive interrupt operation. In response to this complaint, the metric of offload-time ratio, or the amount of time for which a node spends collecting data, relative to the amount of time in which that data is offloaded was established and examined in the context of this work.

The TEMPO 3.2 system, at its worst, had an offload-time ratio of about 7.5, meaning that if 7 and a half hours of data were collected by the node, the user would need to wait one hour for this data to offload. In response to the demand of users for more rapid data offload, the TEMPO 4 system provides two possible avenues towards a solution. The first, hardware and firmware driven, provides a more robust and reliable interface for UART communication. The result of this improved UART communication is the ability to reliably communicate data at speeds up to $1 \mathrm{MBaud}$, implying an order-of-magnitude improvement in the offload-time ratio. A second, PC-side direct MMC-driven offload is described briefly during the future directions portion of this work.

\section{Final Design and Layout}

The test-board configuration of the bus-powered USB transceiver configuration is provided in the schematic capture below. Only one revision of this circuit was produced as part of the TEMPO 4 design process as it performed adequately nearly immediately upon implementation. The FT232's on-chip 3.3V linear regulator output is sourced to the USB_VCC connection in order to provide a USB-present indicator signal to the MSP430. In addition the connections between the USB connector and IC, UART communication lines, and minimal decoupling capacitors can be seen below. 

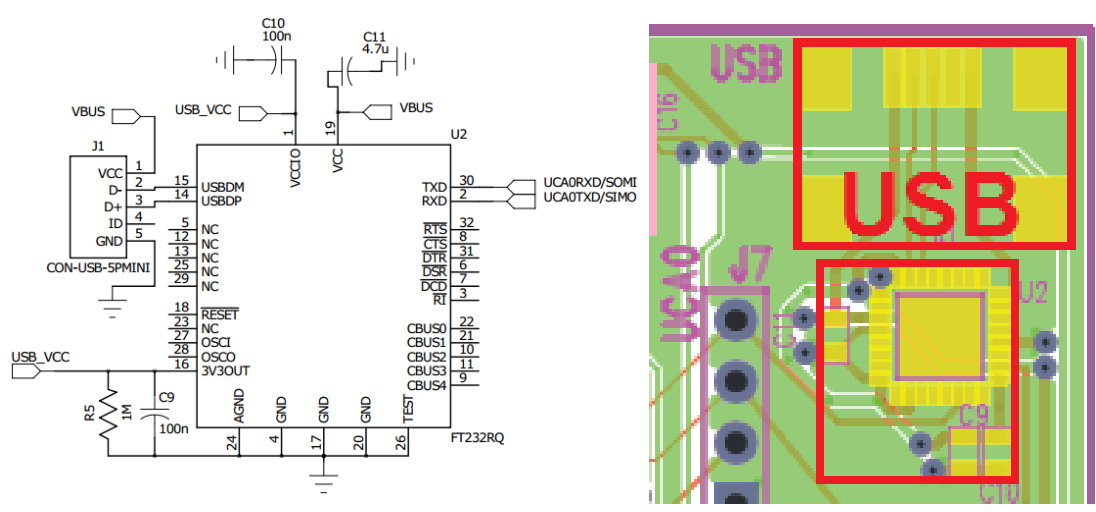

Figure 54: Final TEMPO 4 USB Transceiver Schematic and Layout

In addition to the convenience of having already used a similar part, in an alternate package, as part of a previous TEMPO-related device, the software backend to support basic communication with the chip in Python had also already been established. For this reason, use of a communication interface similar to that of the 3.2 node, but with shorter commands and less communication overheads, resulted in the need for only slight modification of the original Python code. It is hoped that with some careful porting, this newly created communication class might be able to be imported into the existing BodyDATA offload infrastructure created from scratch for the TEMPO 3.2 platform.

\subsubsection{User I/O}

The TEMPO 4 node differs significantly from its predecessors in that it is not necessarily intended for a custom-printed enclosure. Instead, for now it relies on the indiviudal developer to determine the formfactor that best suites the end-point application. For this reason, it was considered advantageous for the TEMPO 4 platform to offer up some ability for direct user interaction. This is accomplished using two optionally populated push-button switches and LEDs for signaling to and from the user.

Generally speaking the concept of push-button and LED based I/O on TEMPO platforms is not new. In fact the grandparent generation to the TEMPO 4 platform, TEMPO 2, featured 2 push-buttons to allow for input along with several LEDs. The TEMPO 3 platform left these same input

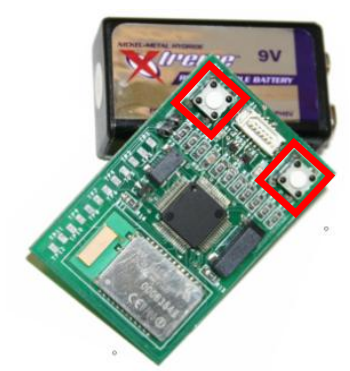

Figure 55: TEMPO 2 Platform with Two User Push-buttons switches out, but maintained the LEDs in order to allow for a water-proof casing, that used translucent material to provide blinking and solid colored indicators to the user.

Since the TEMPO 4 host MCU has programmable, integrated pull-up/down resistors and selectable increased output drive-strength on several ports, only current limiting resistors were added to the LEDs to 
complete the user input design as shown in the schematic capture below. This allows for low area overheads, and minimal impact in do-not-populate cases.

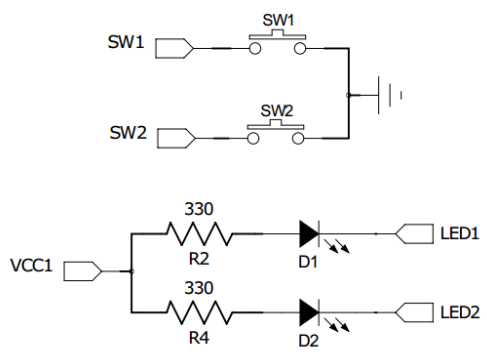

Figure 56: User Push-button Input and Output Schematic

In regard to firmware drivers, simple hardware abstraction-layer (HAL) macros were created to turn each LED on, off, or toggle its state. The push button switches can be monitored either directly, via macros that test for truth of "pressed" and "released" states during synchronous operation, or asynchronously by registering a callback to the pin interrupt by way of a specialized interrupts library.

\subsection{Control, Programming and Interfaces Summary and Conclusions}

After careful consideration of a variety of system controller topologies and commercially available products, the decision to use an MSP430F5342 microcontroller from Texas Instruments is justified using previous design experience, platform and programmability specifications, and interfacing considerations. The MSP430 was first used to develop a robust communications library designed for ease-of-interfacing with a wide variety of commercial sensing, storage, and transmission devices over UART, SPI, and I2C. This communications code was then leveraged towards both the TEMPO 4 development header and the management code for the UART interface dedicated to the on-board USB transceiver IC.

The co-design contributions of this work fall primarily into the categories of MCU management and serial interfacing, as the USB communication portion of this work was already discussed ]explicitly as a case study on the subject. System controller selection and programming interface selection are primarily motivated not by the co-design they will enable, but rather by their ease-of-interfacing and flexibility to operation under a wide set of parameters. Co-design concepts are stressed in the development of an effective control and monitoring scheme for multiple asynchronous hardware peripherals, and user I/O. In addition the selection of a communication interface well-suited to an end-point application's underlying data representation scheme is demonstrated to yield benefits across the board when it comes to operating power and complexity. 


\section{Chapter 6}

\section{Sensing, Storage, and Transmission}

This chapter addresses the challenge of developing for sensing, storage, and transmission modalities in the ULP body-worn context. Though this work will primarily address this issue of inertial sensing in the on-node context, some minimal focus is also placed on the ability of the TEMPO 4 platform to be rapidly extended to include new sensing and reporting modalities.

\subsection{Inertial Sensing and Sensor Add-Ons}

The introduction section of this work provides some preliminary background on the state-of-the-art in inertial motion sensing and the challenges affiliated with human motion capture. This is intended to familiarize the reader with the basics of $3 \mathrm{DoF}$ accelerometer, gyroscope, and magnetometer measurement. This section discusses the subject of on-board IMU part selection and library creation for the TEMPO 4 platform with an emphasis on the impacts on lifetime, form-factor, and flexibility.

\subsubsection{Commercially Available IMUs}

The commercially available IMU market of today is rather diverse, containing a wide array of motion sensor chips and modules, intended for the capture of any desired sub-set of the 9 DoF sensing frame. For the sake of form-factor, the TEMPO 4 platform sought a more tightly-integrated solution than the 3 packages required for inertial sensing in the previous TEMPO platform.

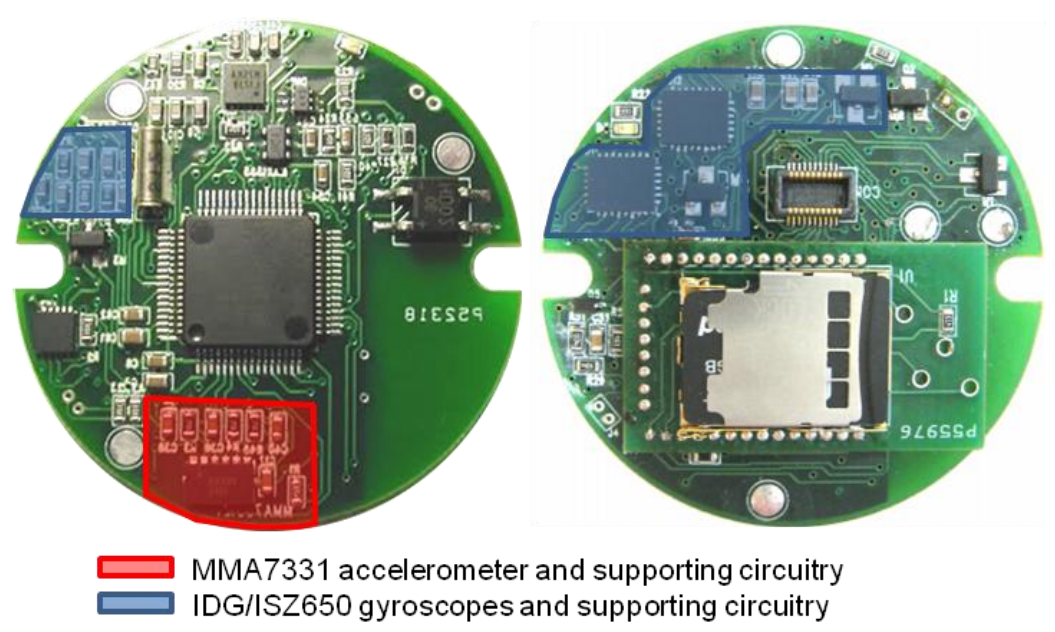

Figure 57: TEMPO 3.2 Board Area Devoted to IMU Solution 
As seen above, the portion of the TEMPO 3.2 system devoted to the accelerometer, and two gyroscope ICs, along with power supply decoupling, analog signal filtering, and header-based power gating, implemented only for the gyro parts, was quite significant. This was justified in nearly all use-cases of the TEMPO 3 system, where inertial motion data collection, was the sole and primary objective. However, as the TEMPO 4 targets greater breadth of sensor deployments, including possibly non-inertial motion capture, the importance of maintaining reasonable form-factor constraints for IMU monitoring in the presence of other system area constraints is crucial.

\section{Area Considerations}

In regard to the state-of-the-art in low form-factor IMUs there are several key competitors. IMU modules are typically small PCB-based device which commonly make use of several commercial IC, a host-controller interface, and a board-compatible footprint to accomplish multi-modality motion sensing in a developer-friendly form-factor. However, more recently as larger silicon manufacturers have pushed to create their own motion-capture platforms, the level of integration is the field has skyrocketed. As a result, even the smallest of these modules, such as the

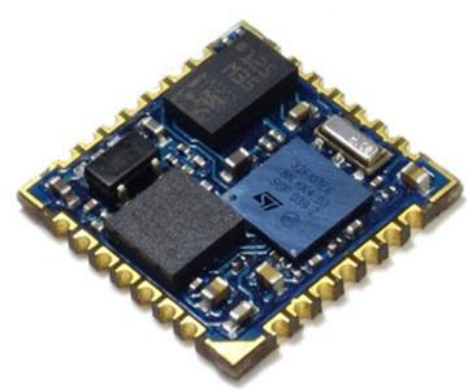

Figure 58: 13x13mm iNEMO 9DoF IMU Module from ST Microelectronics [47] 13x13mm iNEMO platform from ST Microelectronics [47] have been displaced by their smaller form-factor all-IC based alternatives.

The state-of-the-art in today's multi-modality IMU IC market is rather impressive. Analog Device, Invensense, and ST Microelectronics are currently all delivering 6 and 9 DoF motion sensors in a variety of packaging. Possibly the most impressive efforts in form-factor integration of an IMU up to this point lie between Invensense and ST Micro, who are both packaging full 9 DoF parts in 3x3mm low pin-count packages [48]-[49].

\section{Power Considerations}

As previously referenced in this work, the gyroscopes on the TEMPO 3.2 platform consume about $55 \%$ of system power when left on during a flash-based data collection. This means that nearly half of the charge stored in a TEMPO 3.2 node's battery is dissipated in the gyros during device

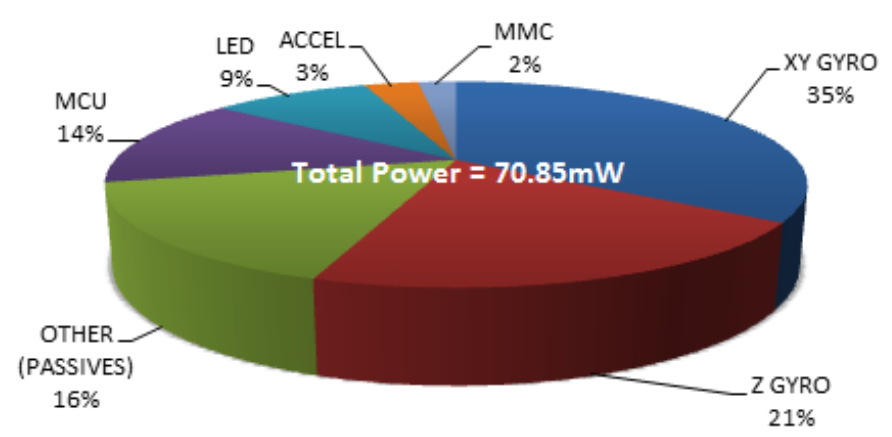

Figure 59: TEMPO 3.2 System Power Budget 
operation. Working backwards from average system power to current consumption using the system operating voltage of $3.3 \mathrm{~V}$, it can be found that the gyros on board TEMPO 3.2 required an average current of about $12 \mathrm{~mA}$ to be delivered continuously to the device throughout operation.

One major motivator and goal of this work is bringing the TEMPO platform up to the state-of-the-art by implementing a new, lower-power gyroscope solution designed to enable longer 6 DoF motion capture sessions. The method by which this power-gain is accomplished is primarily through the selection of a more intelligently managed, digitally interfaced sensor capable of reducing active powers both through improve MEMS sensor element design and efficient electrical management of the gyros during inactive periods. Fortunately, this happens to be where the Motion Processor Unit (MPU) series multi-DoF IMUs from Invensense truly excel.

Invensense has long had its hand in the development of low-power PCB-mountable gyros for high precision signal capture. In fact, both the IDG and ISZ650 dual and single-axis gyroscopes used in the TEMPO 3 platforms were designed by Invensense. As a result, while accelerometer and magnetometer average currents stagnate in the range of $1-300 \mathrm{uA}$ and gyro power continues to be a dominant challenge in the design space, Invensense has a significant leg up. As a point of comparison, while the ST Micro LSM330 6 DoF motion sensor's gyros claim to draw about 6.1mA during operation, the MPU9250 from Invensense is capable of implementing a $9 \mathrm{DoF}$ sensing solution in the same footprint, and reduce gyro power to about $3.2 \mathrm{~mA}$ for all 3 axes. The result is nearly $50 \%$ reduction in the gyro power budget and a $44 \%$ reduction in overall IMU power budget in the 6 DoF use case.

\section{Flexibility and Interfacing Considerations}

Most of the ultra-small form-factor, commercially available IMUs interface the host-controller through a serial digital interface. This digital interface can often be dual-function. On one hand it is intended to simplify driver development and host-controller constraints implied by adding the device to an existing design, reducing the need for solutions with complex ADC topologies or precisely timed sample measurement. On the other hand, it also allows silicon designers to better integrate custom, lower-noise, and often lower-power electronics close the MEMS elements in order to precisely control and monitor the device for irregular operation.

As a result of this trend toward digital interfaces in the IMU community it was suggested that the TEMPO 4 platform make use of its rigorously developed and tested communications libraries for the sake of interfacing the on-node IMU as well. Since both the ST Micro and Invensense IC offerings discussed thus far in this sub-section can both interface with either SPI or I2C, it was decided that the communication 
libraries discussed earlier in this document would form the basis for the on-board IMU management interface.

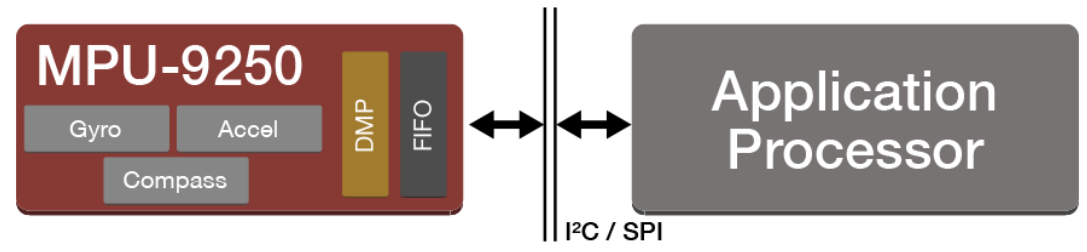

Figure 60: MPU9250 9 DoF Motion Capture Platform with I2C or SPI Interfacing [49]

Last, but not least, the potential ability of the system to expand or reduce its sensing modalities to better fit the fidelity and lifetime constraints required by a particular deployment was considered as a significant benefit for device within the candidate sensor IC pool. While nearly all products allowed for selective power-gaiting of IMU devices that were not in use, few offered the added benefit of cost-selective ordering through the use of multiple degree-of-freedom sensing platforms all integrated into a common, pin-compatible footprint

\subsubsection{IMU Product Selection and Device Testing}

Based on the results of surveying the commercially available IMU market space, it was decided that the MPU-6XXX series single-IC 6 DoF IMU would be used as the on-board inertial sensing platform for TEMPO 4. This decision was based upon the leading-edge form-factor and power constraints of the MPU series along with the pin-for-pin compatibility with the slightly more expense and more capable MPU9150 platform.
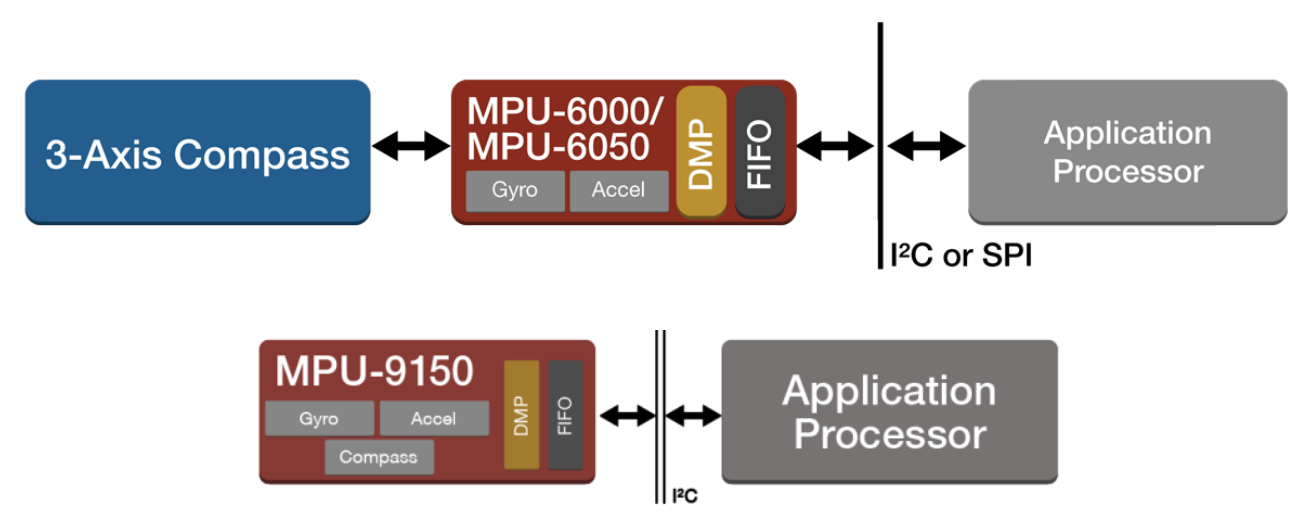

Figure 61: Pin-compatible MPU6xxx and 9150 IMUs in 4x4mm QFN Package [50][51]

Both the MPU6050 and MPU9150 platforms have the advantage of small area overhead implied by the chip itself. This small device footprint is enabled by the ICs' low-pin count I2C interface and minimal additional off-chip components. The result is a single pin-out for all MPU series IMUs with I2C 
interfaces in a 4x4mm 24-pin QFN package, implying a rather low total area dedicated to sensing on the TEMPO 4 final layout.

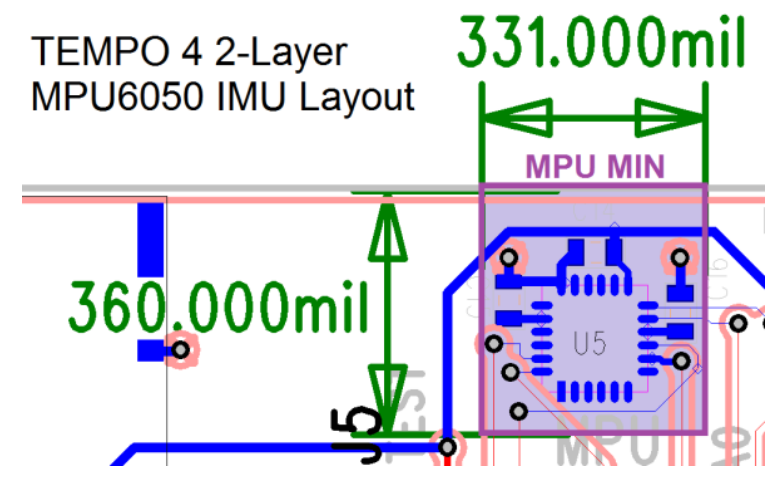

Figure 62: Area Overhad Affiliated with MPU6/9xxx Motion Sensing

At the beginning of this design process the MPU 9 series was not available for use so the MPU 6 series was selected instead. To begin with, the MPU6000 device from this family was selected for use in this design. The primary motivating factor for use of this part over its close relative, the MPU6050, was that of its interface. While the 6050 offered an I2C-only interface for the purpose of data interfacing the MPU6000 implemented a dual-purpose SPI/I2C interface supposedly capable of using either protocol. Thus, since an on-board interface would already need to be devoted to MMC interfacing, which is discussed later in this chapter, it was decided that the MPU6000 part would be used in SPI mode for preliminary evaluation.

Though these earlier chips seemed more capable and varied in their interfaces, no commercially available breakout modules were available for the MPU6000 IMUs. Thus, for the purpose of further evaluating this platform the following minimal breakout board for the 6000 product was designed to mimic a more popular, well-used breakout for the device's 6050 cousin.
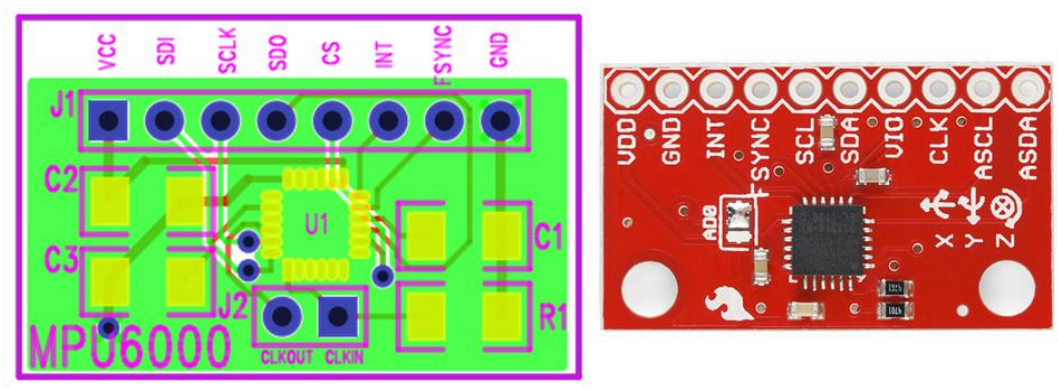

Figure 63: Custom MPU6000 Breakout and SparkFun MPU6050 Relative [52]

The MPU6000 test board was evaluated on the bench using a variety of well-tested MSP430 platforms used throughout the early development cycle for operating code and library development. However, 
regardless of supply conditions and sensor interfacing, all of the populated MPU6000 breakouts were unresponsive. After rigorous hardware debugging and even contacting Invensense to order a new round of ICs (they had since been removed from the distributors website), no progress was made in successfully developing any working interface with the MPU6000. This is a key demonstration of the value of iterative co-design concepts application in the product development cycle. Had this piece of hardware made it into the final TEMPO 4 test board without isolated firmware testing, a great deal of time may have been spent attempting to debug the much more complex power network or serial communications taking place on the full-system test platform.

Instead, midway through the platform development cycle the decision was made to move over to an I2Cbased IMU communication strategy. This was largely based around the implied market direction, as Invensense has since announced they will only offer products with an I2C interface, and time-constraints implied by the duration of this work. The system-level impact of this decision is that the USB transceiver and MMC are given their own dedicated UART and SPI connections respectively, while the MPU6050 is attached to USCI B1, which now serves as only an I2C-only interface, with the default address of the 6050 reserved to avoid bus collision. This does not invalidate the fundamental goal of achieving UART, SPI, and I2C communication all in the development header as USCI A1 is still available as a fully configurable UART/SPI interface.

One added benefit of moving to the MPU6050 and I2C-based communication was that it enables the previously mentioned pin-for-pin compatibility of the TEMPO 4 platform with a variety of 3,6, and 9 DoF motion sensors all packaged in the same 4x4mm QFN. In addition, once moving to the MPU6050 platform, the MSP430 was able to communicate with the device almost immediately. Using the I2C portion of the serial communication library, a thin driver was developed to allow for simple developermanipulation of device sleep and sampling parameters. The result is a fully functional 6 or 9 DoF IMU solution with little-to-no added complexity on top of that of the core serial-interface management code.

\subsubsection{Sensor Add-ons}

In addition the on-board inertial storage discussed the remainder of this section a top-board designed for electrocardiogram (ECG) signal capture using TI's ADS series flexible, bio-electrical Analog Front-End (AFE) was developed, but not produced to demonstrate ease of interfacing new sensing modalities to the existing TEMPO 4 node. The hardware design for this board was based heavily upon the recommended operating circuit provided in the data sheet and was completed in about two days. 


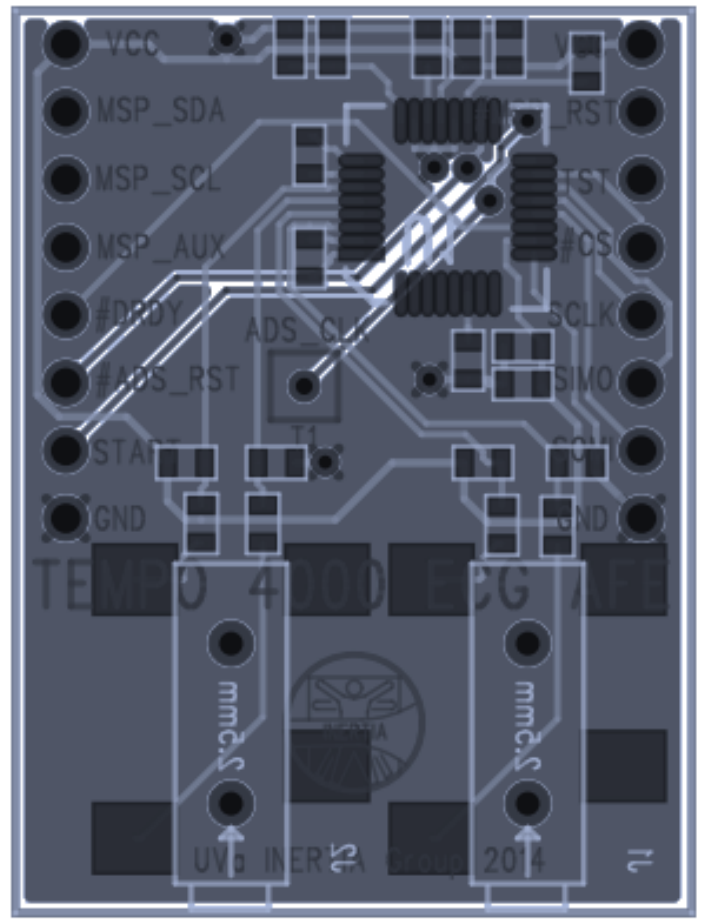

Figure 64: TEMPO 4 ECG Top Board Layout

This ECG top-board makes use of two differential channels, each terminated with a $2.5 \mathrm{~mm}$ headphone jack, a somewhat widely available lead termination style. Since the AFE is interfaced entirely over SPI and has programmable gain, sampling rate, and channel configuration it is worth noting that this board could also be used for monitoring a variety of differential bio-signals in the future, including electromyogram (EMG) and electroencephalogram (EEG).

\subsection{System Storage}

In response to incredibly positive feedback regarding the extended deployment capabilities of the TEMPO 3.2F system, and relatively few complaints about the lack of immediate feedback of data to the user as a result of flash storage, it was decided that support for on-board MMC interfacing was nonnegotiable. A great deal of debate eventually formed around the subject of wireless transmission and usability versus form-factor and lifetime constraints. For the purpose of this document, this section will assume on-board flash memory is to be used in the TEMPO 4 system, with or without additional support from wireless communication. The following section contains a more in-depth discussion of the topic of wireless communication in regard to form-factor, lifetime, and flexibility. 


\subsubsection{Storage Form-factor and Lifetime Constraints}

As previously implied, the significant reductions in system power achievable through use of implementing high-capacity memories on-node can dramatically outweigh the costs of not being able to provide users immediate data feedback in some use cases. The TEMPO 3.2F platform successfully leveraged several such cases to great avail. In order to evaluate the lifetime constraints implied on a system by its on-node storage a typical data rate for the TEMPO 3 system's sensor front-end is determined as follows.

$$
R_{\text {data }}=128 \frac{\text { samples }}{\mathrm{s}} * 6 \frac{\text { axis values }}{\text { sample }} * 16 \frac{\text { bits }}{\text { axis value }} \approx 12.2 \mathrm{kbps}
$$

Thus, we can approximate the data lifetime of our system, or the amount of time for which a given storage medium can buffer data without need for offload as:

$$
T_{\text {data }}=\frac{C}{R_{\text {data }}}
$$

Where $\mathrm{C}$ is the capacity of the memory in bits, and $\mathrm{R}_{\text {data }}$ is the rate at which data is produced in the system. Based on preliminary determination and rudimentary estimation of system operating parameters it was decided the TEMPO system would likely not be able to operate beyond 7 days on a single charge given most acceptable battery chemistries and volumes. Thus, technically speaking $\mathrm{T}_{\text {data }}$ need not exceed 7 days. However, it is common for both commercial and research platforms to significant over specify the data lifetime of system relative to battery lifetime. This is assumed to be primarily for the purpose of allowing for multiple data sessions to take place between two charge periods without the need for intermitted offload. In order to set a reasonable lower-bound for the data capacity of the TEMPO 4 system storage a maximum lifetime of twice that of the battery lifetime is determined to provide a minimum total storage capacity of around 14 days worth of data. Working backwards through the previously provided expressions we find the affiliated storage capacity to be around 2GB worth of data.

The previously arrived at storage number is a familiar one, as it is the same lower-bound used by the TEMPO 3.2 system for its on-board memory. Also similarly to its 3.2 predecessor, the TEMPO 4 platform will need to make use of a non-volatile memory topology, as the device cannot supply power to maintain the state of the memory during long idle periods. However, since the TEMPO 3.2 node had been produced several key innovations had been introduced into the memory market place, and thus a survey of the state-of-the-art in integrated memory solutions was conducted. Rather than include the full results of this survey in this document it will be summarized with three general conclusions below. 
1. Small form-factor, serially-interfaced non-volatile and volatile storage ICs are common solutions for low-capacity memories. However, as the size of a non-volatile memory grows towards $1 \mathrm{~GB}$ and beyond low foot-print parts become far less common.

2. While the use of experimental non-volatile topologies, particularly ferro-electrical RAM (FeRAM) and spin-based solutions, hold significant promise for the future of low-power, high-density memories, many of the products of today do not feature large enough capacities or significant data robustness.

3. In the arena of widely available, low-cost, low form-factor flash memory storage solutions the microSD card is about as tightly integrated as nearly all significant silicon competitors.

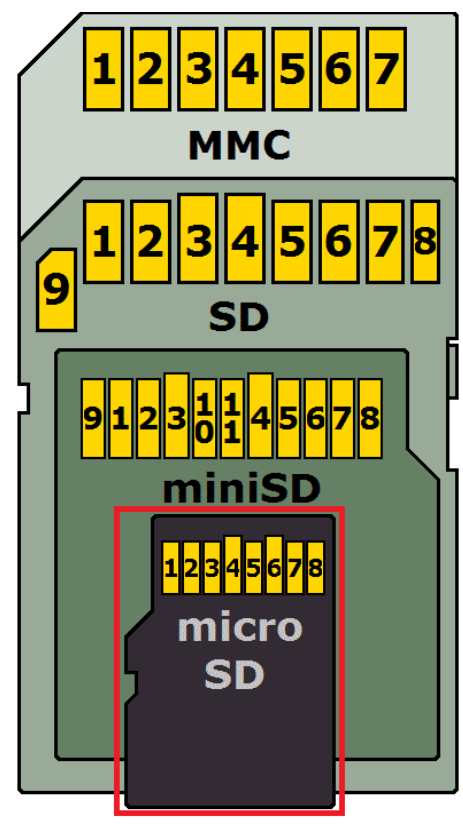

Figure 65: The MMC/SD Form Factors [53]
The final conclusion summarized above is possibly the most significant in the TEMPO 4 system storage decision. With unit costs below $\$ 5$ and net areas just larger than some of the smallest Ball-Grid Array (BGA) packages featuring larger, more complex memory controllers the microSD form-factor was determined to still be on the of most competitive flash-based solution currently on the market.

\subsubsection{MicroSD and MMC Interfacing}

The MultiMediaCard (MMC) standard is a NAND-based flashmemory system commonly used in multimedia applications. Though the original card specification requires a 1-wire serial interface [56] it is now common for devices to use 2, 4 or 8 bits of parallel serialized data for offload [55]. From the year 2000 onward

the Secure Digital (SD) Association has come to represent a significant portion of the MMC marketshare. Though SD does offer some additional functionality on top of basic MMC storage it will not be discussed as a part of this work.

Generally speaking each SD/MMC device has a 7-9 pin footprint, but we will focus on the 8-pin microSD form factor for discussion here. This is both a result of its low form-factor, and high commercial availability as it has more recently found wide-use in the consumer electronics market as a primary source of storage for digital cameras, cell-phones, handheld gaming devices, and even mp3 players. One reason 
for this rapid adoption into the commercial market is certainly the dual-interface model supported by the cards, providing a simple single-wire mode for serial communication and a more full-featured parallel transfer mode for applications that demand higher speeds.

Both the SD and microSD form factors feature two modes of operation:

- SD Bus in which a single bi-directional command line and one/multiple bi-directional data channels are used to transfer data

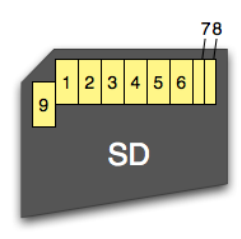

\begin{tabular}{|c|c|c|}
\hline Pin & SD & SPI \\
\hline 1 & CD/DAT3 & CS \\
\hline 2 & CMD & DI \\
\hline 3 & VSS1 & VSS1 \\
\hline 4 & VDD & VDD \\
\hline 5 & CLK & SCLK \\
\hline 6 & VSS2 & VSS2 \\
\hline 7 & DAT0 & DO \\
\hline 8 & DAT1 & $\mathrm{X}$ \\
\hline 9 & DAT2 & $\mathrm{X}$ \\
\hline
\end{tabular}

- SPI Bus in which a standard SIMO,SOMI, SCLK, and CS structure is used to transfer data and commands to/from

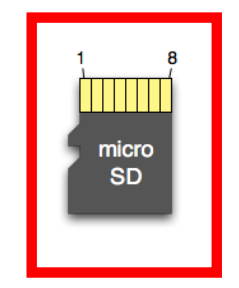

\begin{tabular}{|c|c|c|}
\hline Pin & SD & SPI \\
\hline 1 & DAT2 & X \\
\hline 2 & CD/DAT3 & CS \\
\hline 3 & CMD & DI \\
\hline 4 & VDD & VDD \\
\hline 5 & CLK & SCLK \\
\hline 6 & VSS & VSS \\
\hline 7 & DAT0 & DO \\
\hline 8 & DAT1 & $\mathrm{X}$ \\
\hline
\end{tabular}
the card

It should be noted that the microSD card has two unused (unconnected) pins in the SPI configuration (labeled as DAT1

Figure 66: SD/microSD Operating Modes [54] and DAT2 in Figure 66). These are the parallel data transfer lines which are not considered as a part of this SPI implementation, but are used to improve offload efficiency when running in the full MMC standard supported by many consumer electronic devices such as personal computers. It is worth noting that though the card is written through a 1-wire interface on-node, it can be offloaded via this parallel transfer to significantly improve offload times when large amounts of data need be collected without intermittent offload.

\section{Common Sockets and Connectors}

The MicroSD form-factor can be connected to using a variety of styles of connectors (sometimes referred to as sockets) available through common distributors such as DigiKey or Mouser. There are 3 classes of connectors that are commonly used. These are summarized in Table 16 below. 


\begin{tabular}{|c|c|c|c|}
\hline Connector Name & Description & Example Part & Image \\
\hline Push-Push & $\begin{array}{l}\text { Push card in until } \\
\text { it locks to insert, } \\
\text { push in until it } \\
\text { pops out to } \\
\text { remove }\end{array}$ & Hirose DM3AT & \\
\hline Push-Pull & $\begin{array}{l}\text { Push card in to } \\
\text { insert, pull card } \\
\text { out to remove }\end{array}$ & Hirose DM3D & \\
\hline Hinged & $\begin{array}{l}\text { Open hinge and } \\
\text { slide card in to } \\
\text { insert, open hinge } \\
\text { and pull card out } \\
\text { to remove }\end{array}$ & $\begin{array}{l}\text { JAE Electronics } \\
\text { ST1W008S4FR2000 }\end{array}$ & \\
\hline
\end{tabular}

Table 16: Summary of Available microSD Connectors

In addition to the 8-pins provided to/from the MMC card,

which are sourced to the board through pads on the connector, most connectors also provide what is referred to as a Card Detect (CD) control. These line(s) are used to represent the presence of a microSD card in the connector and are normally connected to a small integrated, mechanical switch.

Figure 67 shows a common microSD connector foot print captured from a schematic using a Hirose push-push connector. The connections to the CD lines are such that the microcontroller, using an internal pull-up resistor and software

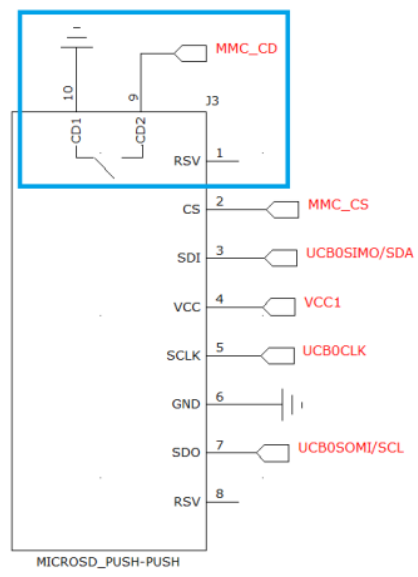

Figure 67: MicroSD Connector Schematic Symbol de-bouncing, can easily detect when a card is present in the system. For the TEMPO 400 design a surfacemount, push-push, microSD connector from Hirose was selected to ease the difficulties encountered when adding/removing cards from the previous TEMPO 3.2F design, which used a less cooperative, hinged connector mounted inside the case.

\section{Power Considerations}

The microSD standard is a convenient embedded data logging form factor as it works at both 1.8 and $3.3 \mathrm{~V}$, consumes acceptable power on read/write, when compared with radio TX/RX power, and has incredibly low quiescent (non-read/write) currents. However, much like radio transmission it does imply some significant constraints on system supply and regulation. 


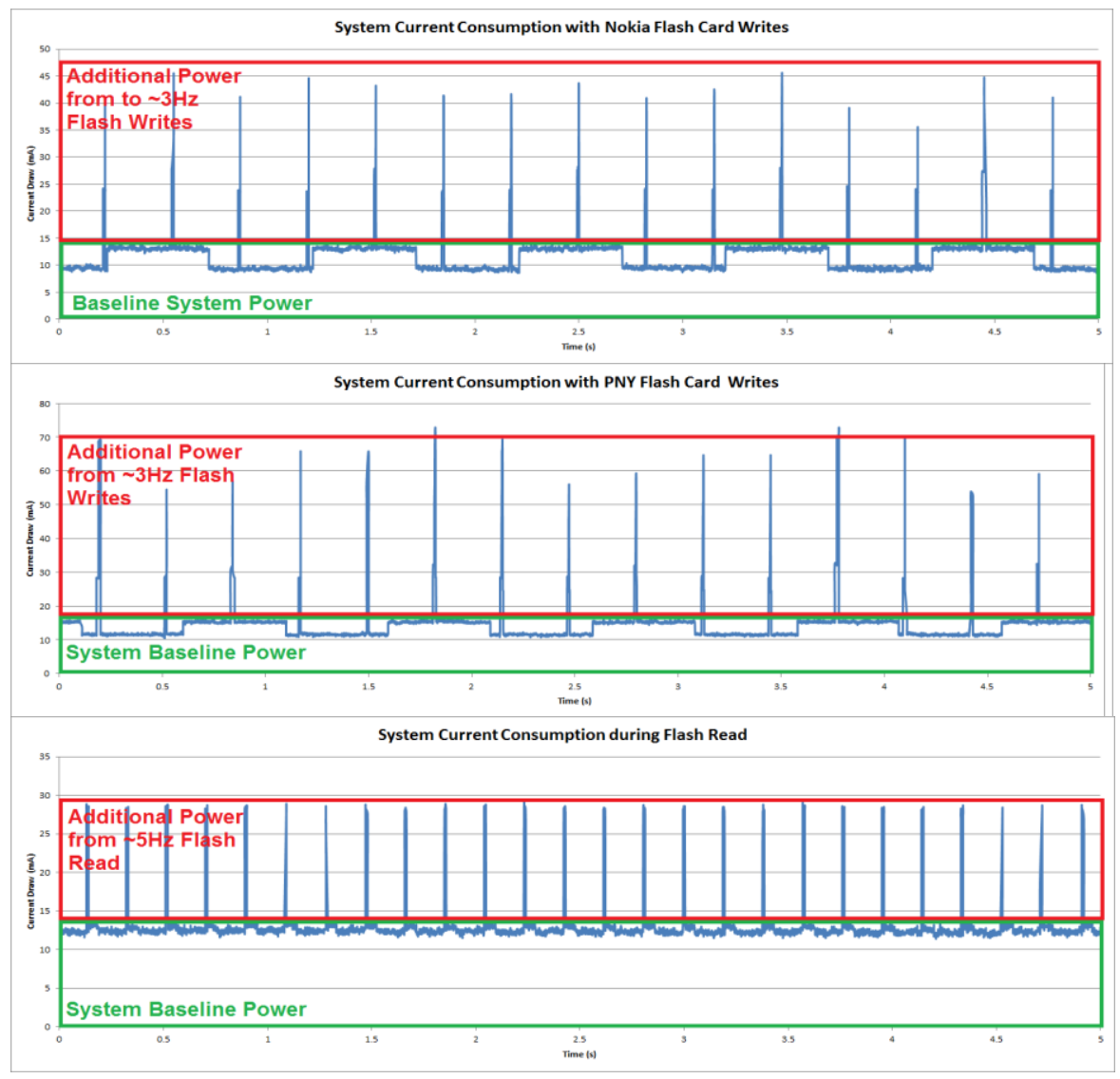

Figure 68: MicroSD Write/Read Currents in the TEMPO 3 and 4 Systems

As seen in Figure 68 the instantaneous current drawn from the system supply by the flash card during write or read operations is quite high, as high as $70-100 \mathrm{~mA}$ in some cases. This means that though average card current consumption will likely be in the 10-100s of $\mu \mathrm{A}$, system supply and regulation will need to be able to supply at least $70 \mathrm{~mA}$ of additional instantaneous current for flash read/write. Since most lithium-based chemistries should never have more than 100mA drawn from them at any point this means efficient decoupling capacitor placement and selection will be critical to reliable, long-term system operation.

\section{Clocking Considerations}

In addition to the power constraints discussed above, it is also worth briefly discussing some minimal clocking considerations for a typical SPI-based MMC setup. Though no dedicated external clock need be provided to the card other than typical master-slave SPI clock pin, commonly referred to as serial clock or SCLK, there are several constraints on what baud rates can be used for commands/data transfers.

Card initialization must occur at a lower rate than the remainder of card operation. For the purpose of initialization a baud rate of $<400 \mathrm{kHz}$ is required. However, once initialized the baud rate can be 
increased significantly, up to 100's of MHz in some cases. In these instances hardware layout must include careful consideration for signal coupling and switching noise. Fortunately, the MCU selected for this design supports SPI baud rate up to $1 / 2$ of the main clock rate, implying in a maximum SPI baud of $16 \mathrm{MHz}$, effectively eliminating the need for special consideration of the SPI bus traces.

\subsubsection{Library Hardware Testing}

Once, the MMC interface was fully specified the firmware design portion of the work occurred rather quickly, using a previously referenced development platform and old TEMPO 3.2 MMC libraries for validation of correct SPI operation. In order to expedite the development and testing of these libraries prior to completion of the TEMPO 4 test board hardware a simple development board-based solution was adopted. By programming the in-system MCU, inserting a flash-card into the on-board push-push connector, and probing the signals between the MCU and microSD directly the code was debugged and validated across a variety of supplied microSD cards.

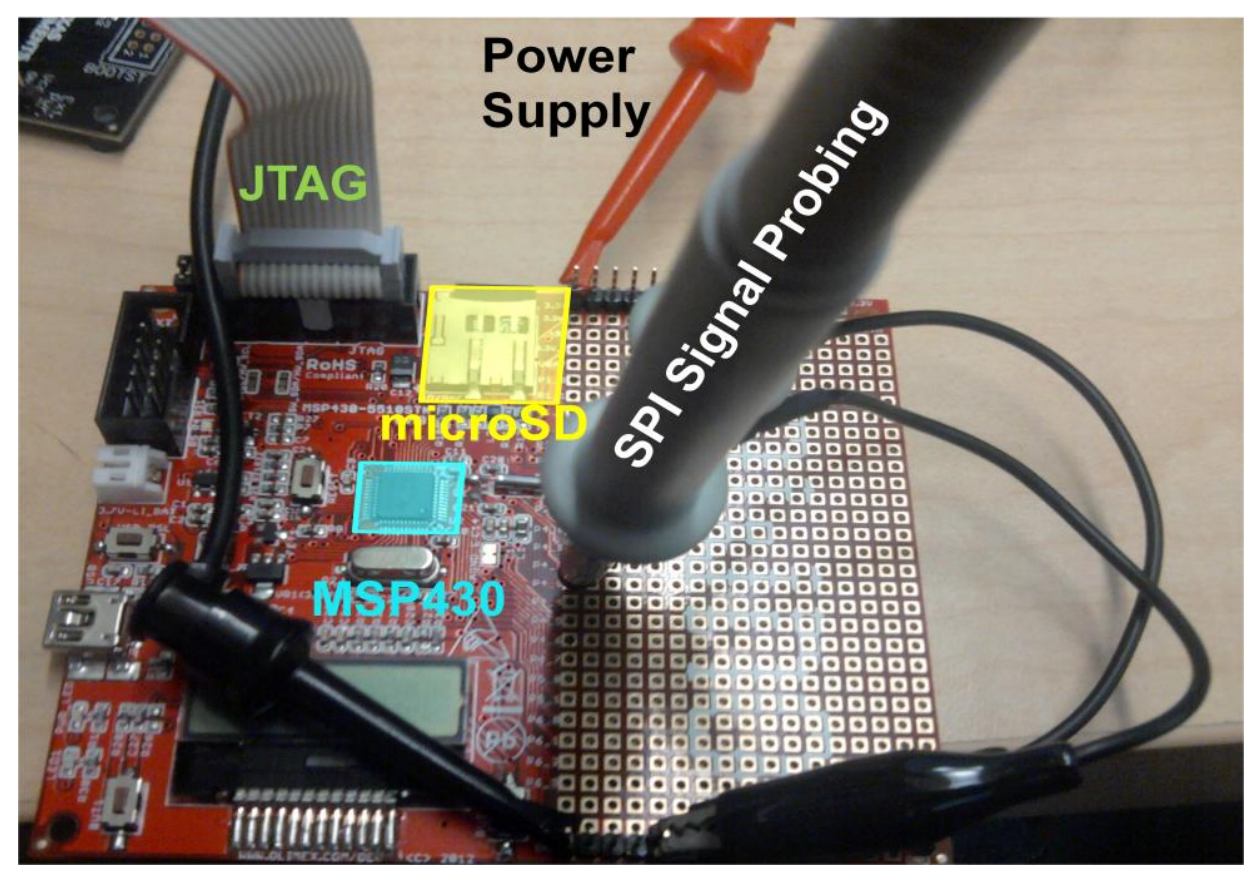

Figure 69: TEMPO 4 MMC Hardware Test Bench with Supply, Programming, and Probe Connections

\subsubsection{Efficient File-Systems for Streaming Data-Storage}

In the plot shown in Figure 68, it may be observed that the system flash-writes are spaced evenly and occur relatively infrequently, about three times a second. This flash management strategy is intended, as per the battery management discussion included in this document, the bursty use of large instantaneous current consumers is not recommended for Lithium-powered systems. 
Though a significant amount of though and effort was put into defining a custom file-system for the TEMPO 4 platform it will only be described briefly here as it primarily represents a small improvement over the already established TEMPO 3.2 custom file system.

The basic idea behind the TEMPO 4 file system is that of ease-of-access, and again, minimization of the amount of controller interfacing and management required for use of the file system. For this reason, many commonly available commercial standards such as FAT FS or NTFS are not well suited to resource-constrained operation. Borrowing from its predecessor, the file system makes use of several types of information "sectors", conveniently delineated by the natural sector length of the card. Unlike the TEMPO 3.2 system's look-up based file system the TEMPO 4 file system uses a linearly navigated linked-list to greatly reduce MCU burdens during flash resume and halt operations, and ideally improve long-term flash card lifetime through better built-in wear-leveling. The TEMPO 4 files system makes use of only three sector types: node information, session information, and data. All sectors are signed with a two byte type-code as well as a two-byte firmware-computed CRC to assess validity. Each of these three sector types and their contents is summarized below.

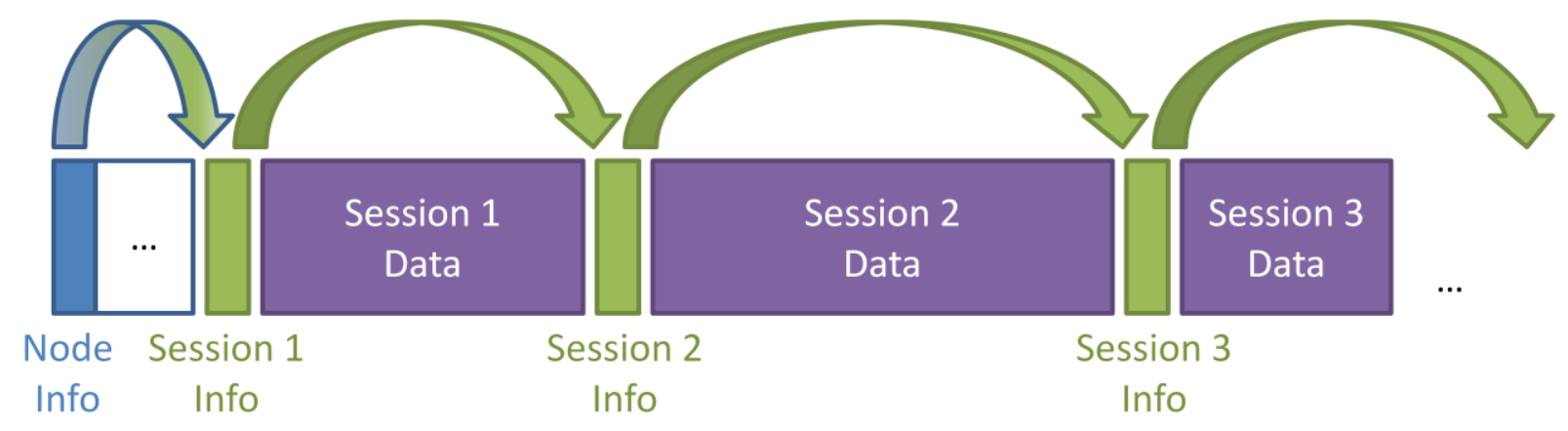

Figure 70: TEMPO 4 File System Linked-List Implementation

There is only one node information sector, in the default TEMPO 4 firmware it falls at the 0 sector address of the card, though it could be programmed to sweep from 0-N to implement a wear-leveling scheme in the future. This node info sector contains general information about the current state of the node, configuration of the sensors, and pointers to the first session info and data sector on the card.

There can be any number of session info sectors on the card, each denoting a particular data session taken on the node. Before any data can be written to the card via the file system's data write function, a session must be started. When a new session is started the previous session's info sector is modified to have its next session info sector value modified to the currently written sector. At this point, a new sector is written containing the previous session info sector value along with the current time from the RTC and a number of other system metrics regarding the session. In this way a traversable linked-list is established. 
Data sectors always directly follow a session info sectors and thus the precise location of the data on the card need not be explicitly stored. The only information other than the four bytes of identifying and verification information contained in any data sector is in fact, data. Users write their data to the output during a collection by simply calling the write data function with a session open and in-progress. The write-data function automatically buffers this data until it has enough information to fill a sector then passes this sector to memory. In addition to the benefit of unmanaged flash writes for developers interested in an easy-to-use interface, the file system offers the ability to use added buffer size to deal with particularly bursty streams of data that might produce large amounts of information for storage in a relatively infrequency manner.

\subsection{Transmission and Wireless Interfacing}

Arguably the biggest source of disagreement amongst technical and non-technical collaborators alike during the TEMPO 4 system design process was that of transmission and wireless interfacing. While many saw it necessary to maintain the tradition of offering up a wireless reporting modality by default in every TEMPO generation, others cited the power and area benefits of flash and specialized routing and population constraints often implied by wireless solutions. Ultimately the design decision made for the TEMPO 4 platform was to include no on-board radio, and this section correspondingly details the motivations, draw-backs, and co-design concepts that lead to this decision.

In order to help better structure the discussion of the lack of inclusion of radio in the TEMPO 4 hardware platform this work will analyze three primary areas of consideration: radio diversity and system specificity, physical overheads and RF design challenges, and ease of developing add-on modules for a variety of radio interfaces via the on-board development interface.

\subsubsection{Radio Diversity and System Specificity}

The primary argument at the heart of the radio decision for the TEMPO 4 platform is that of optimizing platform flexibility. Unfortunately, no one radio solution covers the entire space of acceptable data rates and power consumptions and for any given application or designer, the decision of which protocol optimally suites the needs of the deployment may change. 


\section{Microprocessor and Radio Power Consumption}

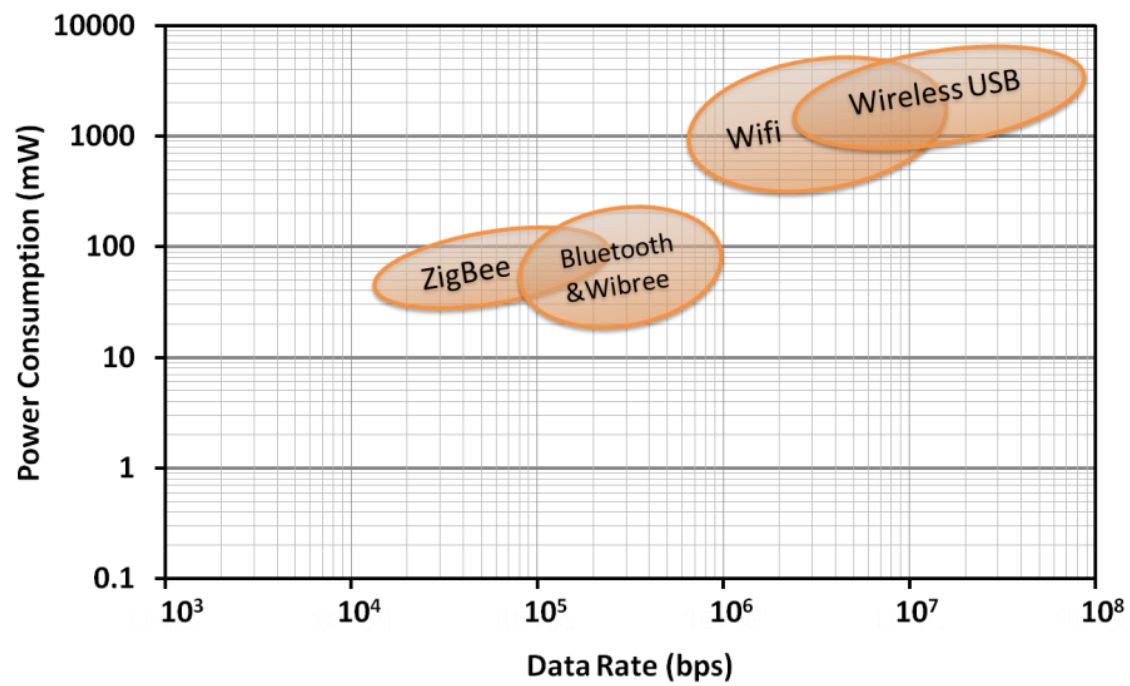

Figure 71: Approximate Power Consumtion versus Data Rate of Several Common Radio Protocols

While designers interested in the more commercially-driven products seek the ease-of-interfacing brought by many common radio standards, it is =common for academic audiences to make use of lesser-known, more specialized wireless signaling techniques for power reduction and increase of reliability. Nonetheless, a wide array of commercially available wireless protocols was investigated to determine primary candidates for ease-of-interfacing and lifetime considerations. The maximum throughput, typical operating power, and primary advantage of each of some more commonly available wireless communication standards is summarized in Table 17 below.

\begin{tabular}{|c|c|c|c|}
\hline Technology & Throughput & Power & Advantage \\
\hline $\begin{array}{c}\text { Bluetooth v3 } \\
3.8\end{array}$ & $\begin{array}{c}240 \mathrm{kbps} \\
\text { (SPP) }\end{array}$ & $\begin{array}{c}\text { Tx: } 280 \mathrm{~mW} \\
\text { Rx: } 180 \mathrm{~mW}\end{array}$ & Interoperability \\
\hline $\begin{array}{c}\text { Bluetooth v4 (BLE) } \\
4.8 \\
\text { Bluetooth }\end{array}$ & $200 \mathrm{kbps}$ & $\begin{array}{c}\text { Tx: } 89 \mathrm{~mW} \\
\text { Rx: } 65 \mathrm{~mW} \\
\text { Sleep: } 1.3 \mathrm{uW}\end{array}$ & $\begin{array}{c}\text { Future Integration, } \\
\text { Low Power }\end{array}$ \\
\hline $\begin{array}{c}\text { Zigbee } \\
\text { ZigBee }\end{array}$ & $250 \mathrm{kbps}$ & $\begin{array}{c}\text { Active: } 165 \mathrm{~mW} \\
\text { Sleep: } 30 \mathrm{uW}\end{array}$ & Mesh Networking \\
\hline $\begin{array}{c}\text { Wifi } \\
\text { Wifi }\end{array}$ & $1 \mathrm{Mbps}$ & $\begin{array}{c}\text { Active: } 100 \mathrm{~mW} \\
\text { Sleep: } 10 \mathrm{uW}\end{array}$ & Existing Infrastructure \\
\hline
\end{tabular}

Table 17: Summary of Widely Available Wireless Communication Standards 
Of the four common wireless standards introduced in the table above, each has its own strengths and weaknesses and seems to have found its own niche set of applications for which it is the best suited candidate for wireless communication.

\section{Bluetooth-based Solutions}

In the recent past, Bluetooth-based protocols have seen significant growth in the consumer electronics space, as cell-phones, personal computers, and even some vehicles begin to support the host-side interface natively. The result has been a huge proliferation of Bluetooth-enabled devices over the past few years. While Bluetooth does a reasonably good job of maintaining small star-topology networks with a limited number of end-point devices, it does not support networks with more than 8 active devices at a time. In addition, its Time-Domain Multiple Access (TDMA) controlled protocol mean that devices need to coordinate with the network on a regular basis to maintain connectivity, this means regular radio usage even during periods where data would otherwise not be communicated, making Bluetooth a bad fit for light or bursty traffic loads.

\section{Zigbee Solutions}

Zigbee represents the standard protocol used for establishing mesh networks in large-scale sensor deployments. Due to its ability to dynamically route packets through the network the Zigbee protocol can achieve output power savings by relying on intermittent nodes for transmission, whereas in Bluetooth all data transmissions are end-point to end-point. Whether or not this meshed style of network transmission is suitable to the on-body context is still a subject of some debate, but it has undoubted demonstrated value already in the areas of structural and environmental monitoring.

\section{Wifi Solutions}

Last but not least WiFi represents the last of the commercial radio protocols considered "common" as part of this work. Though traditionally the high data rates, and corresponding transmit and receive powers affiliated with running a WiFi radio would prove prohibitive for most embedded systems, the high peak data-rates and quick duty-cycling abilities of more recent modules mean in some cases battery-powered WiFi operation is possible. In these situations more work will have to be done to evaluate peak current considerations for long-term battery stability and careful management of system control during transmit and receive windows, but for now it is merely noted that these solutions are becoming increasingly tenable as the Internet of Things (IoT) mentality becomes increasingly pervasive in the hardware design community. 


\section{Less Standardized Solutions}

In addition to the four commonly available wireless standards discussed above there are a number of other, lesser known wireless protocols that are worth mentioning for their own figures of merit. The SimpliciTI [57] and DASH-7 [58] protocols use a Bursty, Light, Asynchronous Traffic model, or BLAST, to significantly reduce radio power when the system has no data to transmit or receive. Meanwhile other standards such as Z-Wave [59] are taking more targeted approaches to efficient RF implementation by tapering designs to a specific context, such as that of wireless home automation.

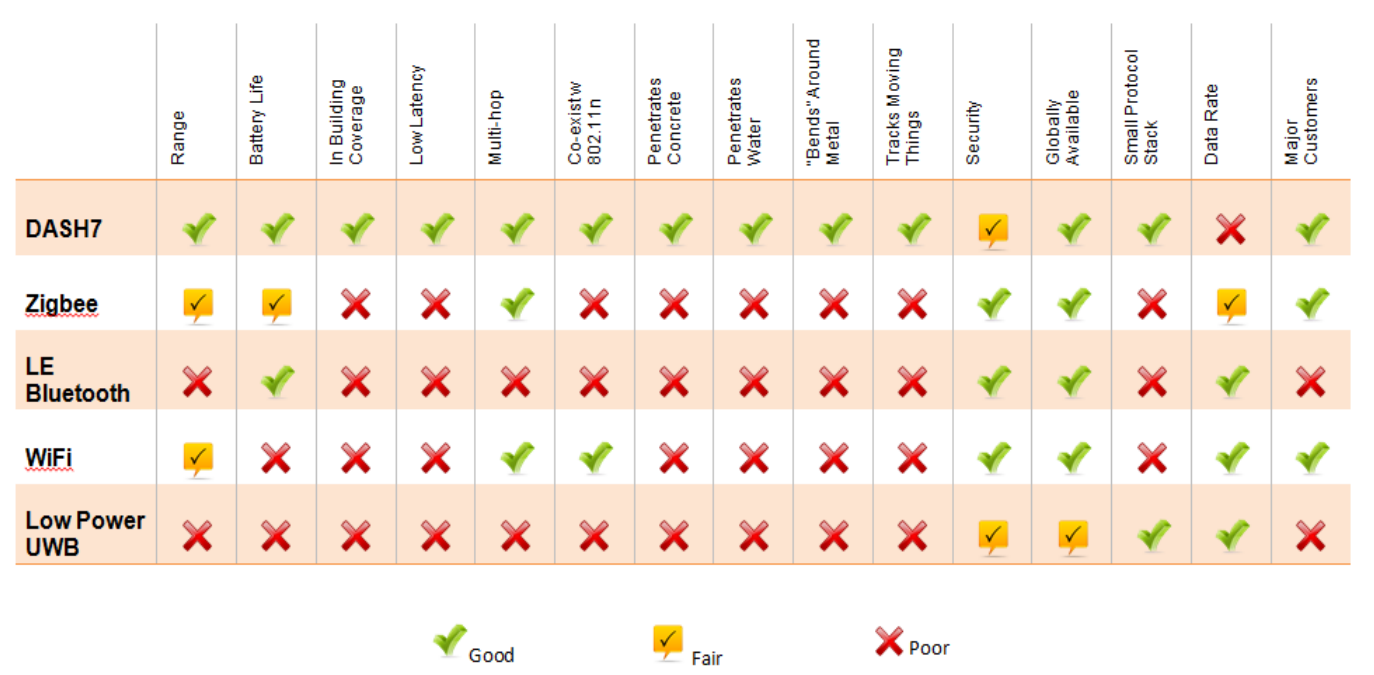

Figure 72: DASH7 Protocol Comparison [60]

The take-away point from this portion of the work is that there is no one-size-fits-all radio solution designed to achieve energy-efficient operation, though many will claim to be. For this reason, in order to optimize the flexibility of the TEMPO platform to various underlying data rates and control structures avenues that exploited multi-functional radio solutions were explored.

\subsubsection{Physical Overhead and RF Design Challenges}

Often the primary consideration that drives system designers away from custom RF solutions is the notorious complexity of signal routing and conditioning in the high-frequency range. In order to avoid the significant challenges posed by custom RF layout, many modern system designers, including previous TEMPO platform architects, make use of pre-certified radio modules to significantly system routing complexity and time-to-design. This can be unfortunate as it prevents many of the flexible radio possibilities alluded to at the end of the previous section. In addition, as the programmability of a final radio solution begins to increase, it often trades off this flexibility with large firmware overheads 
affiliated with configuring the system radios and managing timing and control during communication periods.

The TEMPO 3 platform made use of one such radio module for the implementation of its own Class 1 Bluetooth interface. The RN-41 from Roving Networks was used to establish a connection with the aggregator over the Bluetooth Serial Port Protocol (SPP). Based on this positive previous experience in module-based wireless system design it was decided that any solution to be integrated into the TEMPO 4 platform would need to be made available in a pre-certified board-mounted radio

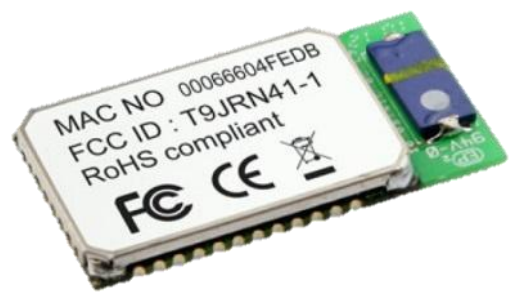
module.

As part of work related to the design of the TEMPO 4 platform, an undergraduate research assistant produced a survey of available Bluetooth modules in the context of physical overhead relative to the 1"x1" form-factor constraint. The survey considered Bluetooth 3 and 4.0 modules from a variety of commercial producers and with varying levels of stack-integration. The top 4 area-sufficient platforms were then prototyped on a custom-designed PCB to be evaluated side-by-side, but this evaluation was not conducted as a part of this thesis. Nonetheless, the area overhead comparison is included below for the sake of reference.
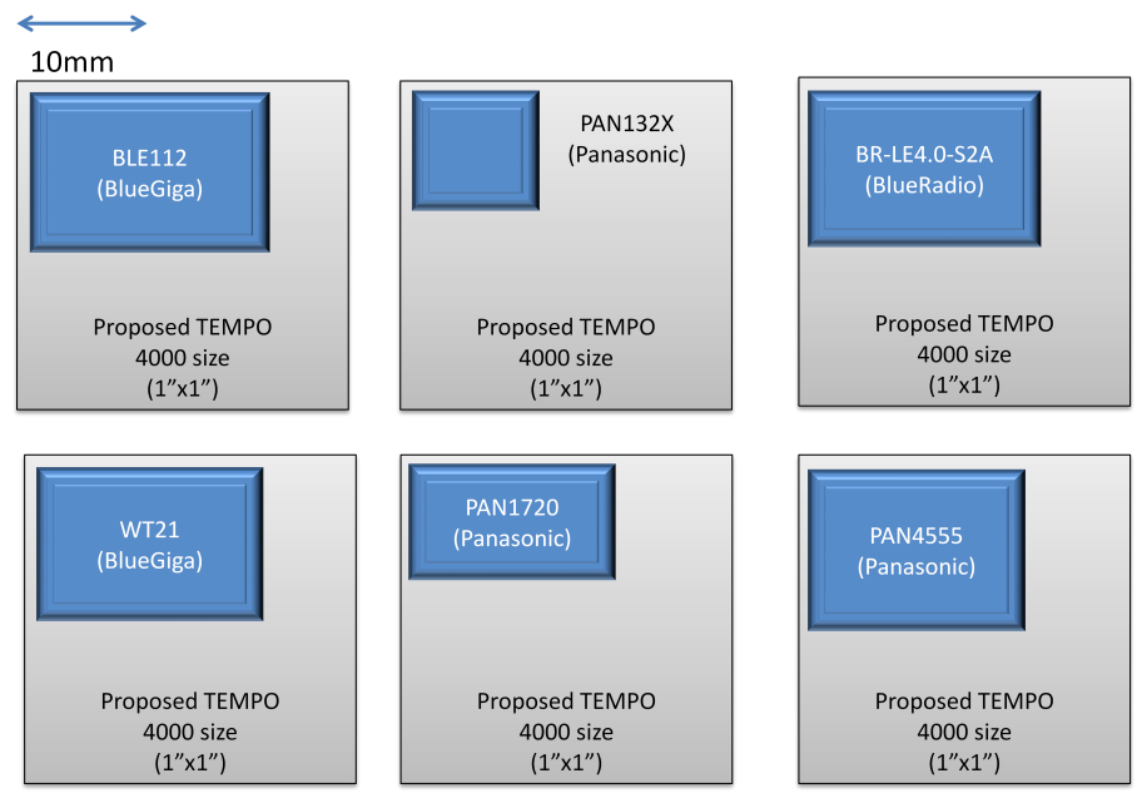

Figure 74: Various Bluetooth 4.0 Module Area Comparison Relative to TEMPO 4 1"x1" Form-factor 
It is worth noting that many of these modules are in fact quite comparable in board area to the flash card holder itself, and also slightly larger or just at, one half of the desired width and height for the bottom of the board. An added constraint to many of these areas that up to this point has been largely ignored is that of RF keep-out. Typically speaking when an electromagnetic signaling mechanism is used to transmit or receive data in the on-board context, the mutual coupling and shielding considerations are crucial for consideration. In order to simplify the process of designing around an RF module, typically the module's designers will specify area around the device that should not include other signal routes. Unfortunately, this is normally specified, not just for one layer of the board but for the entire stack up. The area lost to this keep-out in the TEMPO 3 systems is identifiable as the lighter green areas of the circuit board where the ground plane has not been routed, and thus more light is diffused through the board. This area is labeled in yellow in Figure 75 below.

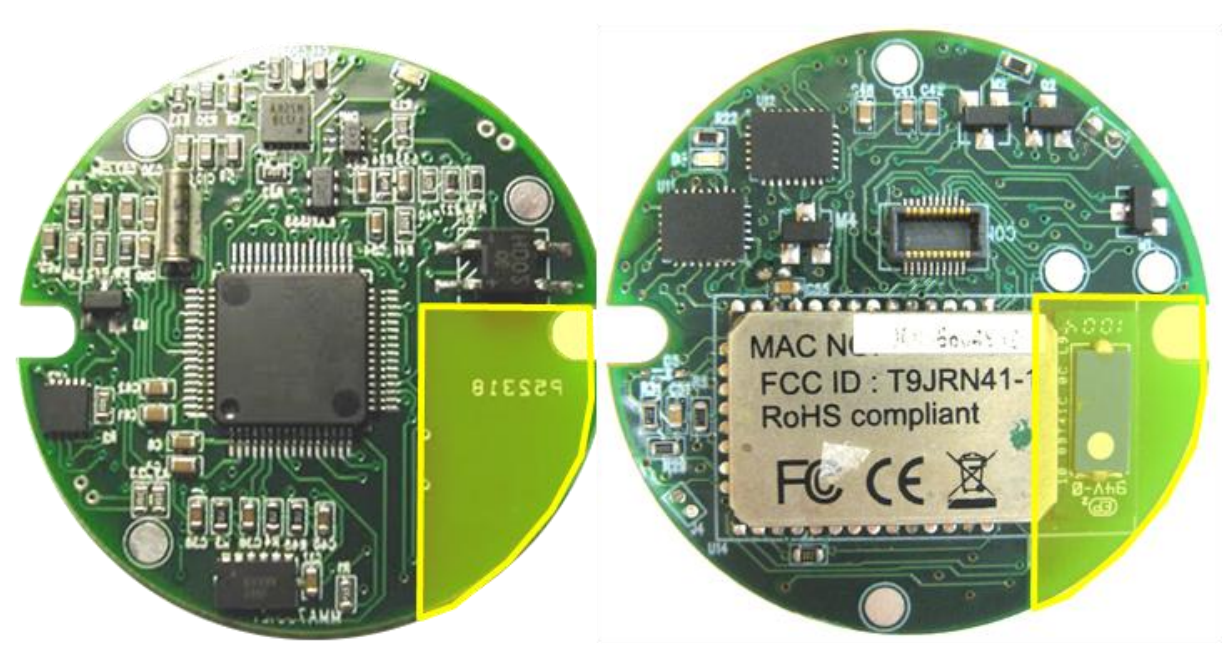

Figure 75: TEMPO 3 Platform with RF Keep-out Indicated

This meant that if a radio module and flash card were to be included in the final TEMPO design the 1"x1" form-factor would most likely need to be violated. The near identical size of the flash card and RF module mean they would likely not be populated on the same layer, and the keep-out generated by the antenna would need to produce clearance constraints for all layers during routing and part placement.

\subsubsection{Expandable Development}

The final motivating factor for leaving wireless communication choices to the user is that of the flexibility enabled by the 16 pin development header summarized in a previous chapter of this work. Since nearly all radios and radio modules of interest implement some form of serial digital interfacing, it is likely that one of the standards offered up in the development header collides with an available module with a similar host-controller interface. This work seeks to demonstrate this point in two ways. First, it introduces a 
wide array of commercially available radio modules implementing common serial interfaces and second it demonstrates the ease of developing a new hardware radio platform through the case-study of designing, but not testing, a BLE radio add-on module for the TEMPO 4 development header.

\section{Serially Interfaced Radio Modules}

This section introduces some common, serially-interfaced radio modules and ICs suggested for possible use with the TEMPO 4 platform. Suggestions are based upon either successful implementation with similar systems in the past, or widely accepted norms for wireless module hardware.

\section{Bluetooth v3.0}

The RN-41 Bluetooth module introduced earlier in this chapter is an excellent candidate for developing on top of the widely commercialized Bluetooth 3.0 standard. Implementing a transparent data transport mode, and a standard AT command set, the module interfaces via UART at up to $115.2 \mathrm{kBaud}$, providing reasonable data transfers rates during use of the SPP.

The RN-41 radio module fits comfortable inside the footprint of the TEMPO 4 development header, and for this reason it is considered probably that a Bluetooth 3 add-on board could be rapidly developed for the TEMPO 4 platform. Based on past experience, by simply connecting the required 3.3V supply lines across the provided $3.3 \mathrm{~V}$ output rails and connecting the minimum of two UART signal correctly Bluetooth functionality should be achieved.

\section{Bluetooth v4.0 (BLE)}

The BR-LE4.0-S2A from BlueRadios [62] is another easily interface-able, compact radio module similar in footprint to the RN-41. It also uses a UART connection and slightly modified AT command set to send and receive data from the user and can achieve data rates as high as $460.8 \mathrm{kBaud}$. In addition the BRLE4.0-S2A is based around the CC2540 BLE System on Chip (SoC), and allows semi-open development for the 8051 platform inside. This is an added benefit as it means the module is also capable of system control in smaller, less complex operating environments.

ZigBee

ZigBee differs from some of the more open-hardware radio standards in that it requires precise control over various hardware parameters in order to maintain network timing and synchronization. For this reason, relatively few commercialized ZigBee radio modules exist. The one, nearly ubiquitous, popular solution is that of the XBee series modules, which implement and easy-to-interface UART module featuring communication rates up to $250 \mathrm{kBaud}$. 
While the XBee module interface is slightly more complicated still than its Bluetooth competitors, it does have the added advantage of additional commands for controlling lower-level network actions that the Bluetooth devices do not feature. For these reasons and more, these modules have up to now primarily found popularity with hobbyists and academics building largely distributed wireless sensing and automation systems where either power or form-factor constraints were not seen as critical.

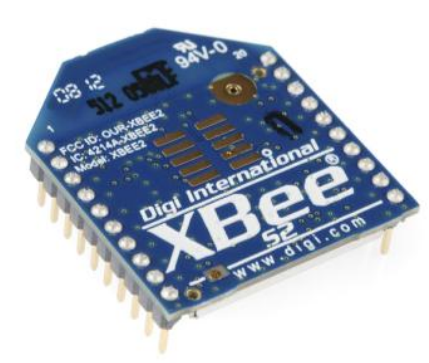

Figure 76: Common XBee ZigBee Shield [63]

However, the tight level of control the ZigBee standard maintains over their hardware solutions has began to be demonstrated as a weak point of their approach. As a variety of large IC companies all vie to produce the next, highest performing, Bluetooth or WiFi specific SoC for use in embedded platforms, ZigBee developers are largely stuck with the older, less power-efficient hardware that has now been on the market for several years.

Nonetheless, the TEMPO platforms power supply network would likely be able to source the $45 \mathrm{~mA}$ maximum current these modules require at $3.3 \mathrm{~V}$. Though these modules are sized just over the formfactor constraint of the TEMPO platform, it may be possible to build a slightly over-sized adapter board for integrating the standard XBee shield footprint's supply and UART signaling with the available connections.

WiFi

The RN-131 802.11 B/G module from Roving Networks [64] is an excellent solution for designers looking for a small-footprint WiFi module for easy project interfacing. In a package just larger than that of the RN-41 and with nearly identical pin-out and UART interface, with much higher affiliated baud, this device could likely also be contained within a custom 1"x1" add-on board for the TEMPO 4 platform.

Unfortunately the instantaneous current draws of most commercial WiFi modules are beyond the specification of the TEMPO system's supply regulation. With a peak transmit current of $212 \mathrm{~mA}$ and a typical transmit current of $140 \mathrm{~mA}$ it is well understood why battery-powered wireless devices rarely implement WiFi interfaces. Nonetheless, if interest arose, the use of a supplemental, external power supply and regulator designed for the WiFi interfacing could enable significant development opportunities in this space.

\section{Alternatives}

Fortunately, a number of commercial RF ASICs also implement common serial protocols. The ChipCon series of radios, somewhat recently acquired by Texas Instruments, were common solutions in the 
900MHz and 2.4GHz band and implemented SPI-based interfaces. In addition more recent RF SoC's with hardware-integrated stacks have begun to revolutionize the wireless design space.

If, in the future, more expertise in the art of RF layout is gained and the TEMPO 4 board-stack topology deemed suitable for noise and cross-talk considerations, then it may be possible to layout a completely customized radio solution entirely contained to a TEMPO 4 top-board. This level of integration represents the ultimate goal of the open design principles discussed in the motivation to this work. Enabling individuals with highly specialized skills and research interest, such as RF system design, to rapidly prototype designs and deploy a platform to other technical and non-technical collaborators.

\section{BLE Add-on Module Design}

In order to demonstrate the straight-forward nature of the development of additional radio platforms, as proposed earlier in this section a brief design was drafted for use of the BR-LE4.0-S2A BLE module described in the previous sub-section. The schematic and layout are included below for reference.

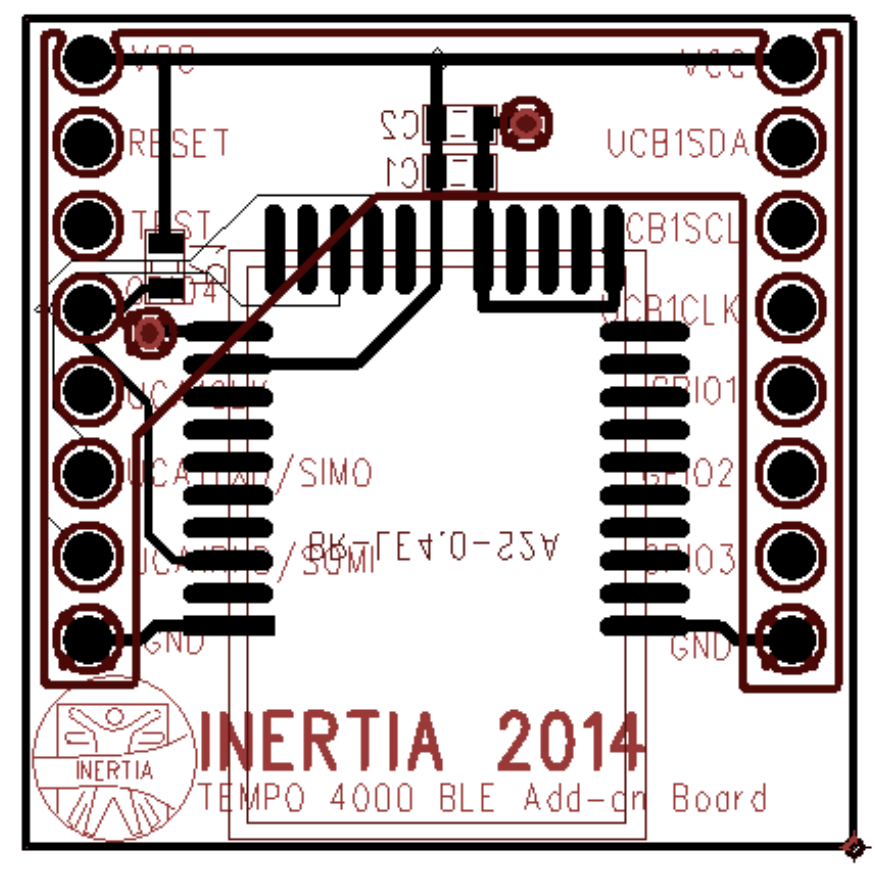

Figure 77: TEMPO 4 BLE Top-board

It can be seen that once the radio parts have been created and the TEMPO hardware libraries imported into the design tool, the hardware drafting is relatively straight forward. The developer simply connects the desired pins together and adds several decoupling capacitors to the radio module to help deliver peak currents during radio transmission. 


\subsection{Sensing, Storage, and Transmission Summary and Conclusions}

As previously referenced, the sensing, storage, and transmission subsystem is designated separately from that of control, programming, and interfaces as it relies on this previous subsystem for constraints. In regard to the constraints implied by the controller selection and interface determination contained in the previous chapter, this work stresses the use of three common serial interfaces: UART, SPI, and I2C, to interface a wide variety of sensing and reporting modalities.

The TEMPO 4 system communicates with a microSD card over SPI-based MMC communication and interacts with the on-board 6 or 9 DoF IMU via I2C on USCI B1, fixing this interface as an I2C in the final realization of the platform. Last, but not least, no singular radio solution is selected for the main reason that it was believed any choice of one particular radio would impact the physical, electrical, and operational parameters of the design enough that it was left to the developer to make the decision of whether or not wireless aggregation will be necessary for their application at design time.

The co-design concepts stressed in this chapter are those of firmware organization to support rapid expansion of new hardware modalities along with the value of efficient, iterative firmware testing throughout the development cycle in discovering potentially non-functional hardware components before they make it into final designs. In regard to MMC operation, the importance of managing system peak currents and the implications of the decision of microSD storage on system timing and signaling are discussed. Last, the challenge of specifying a hardware solution for an unknown application space is deemed to be too significant a leap to justify devoting significant board and code space to any single radio solution. Instead the determination of radio constraints at design-time allows for greater freedom of implementation for system designers interested in cross-hierarchical optimization of what is often one of the largest power consumers in traditional wireless sensor systems. 


\section{Chapter 7}

\section{System-Level Design Summary and}

\section{Analysis}

The TEMPO 4 system is a wearable, expandable 6 or 9 DoF motion capture system for use in a variety of possible data collection scenarios. It was designed in two primary hardware cycles while making use of rigorous, iterative firmware co-design and testing to assure vertically oriented test benches were performing as expected throughout the design process.

\subsection{TEMPO 4 Test Board}

The first major integration effort toward the final TEMPO 4 platform was that of the system test board, provided in Figure 78 at right. This board uses a 2"x2" form-factor to achieve a 2-layer stack-up while allowing for plenty of room for silk-screen documentation, test-points, and opportunities for designer intervention in the case of part misselection or failure.

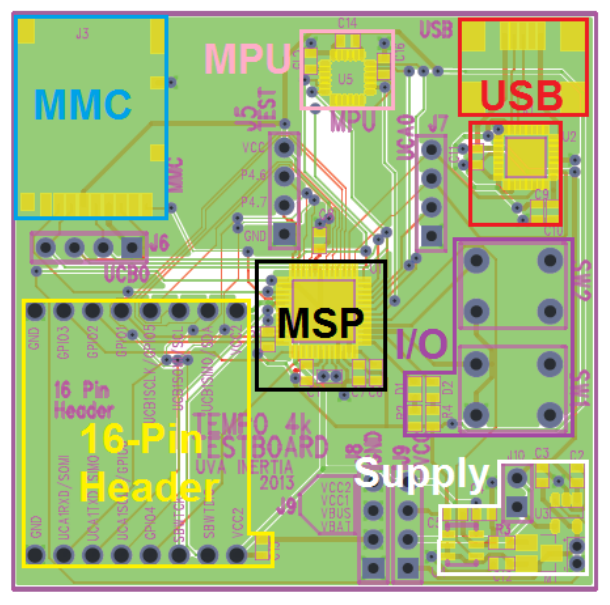

Figure 78: TEMPO 4 System Test Board Layout

Following the manufacturing and population of this test platform the board was evaluated while running a set of rigorous subsystem oriented tests. To begin with, one small problem in the supply regulation portion of the design was found and resultingly, the affiliated supply headers, shown to the left of the supply area in Figure 78, were used for power supply instead. In addition a minor modification of 16-pin development header and USB connector footprints was performed following evaluation of this test platform.

In addition to being used to evaluate the selected parts and their affiliated operation and footprints, this board was also distributed to several undergraduate research assistants hoping to produce the first round of INERTIA-sponsored, internally developed add-on boards. Reviews, suggestions, and improvements regarding the early structure of the communications library and core control code were all accepted and integrated based on their feedback. In some cases, including I2C library development, undergraduates directly contributed small amounts of code or code examples to the core body of this work as well. 


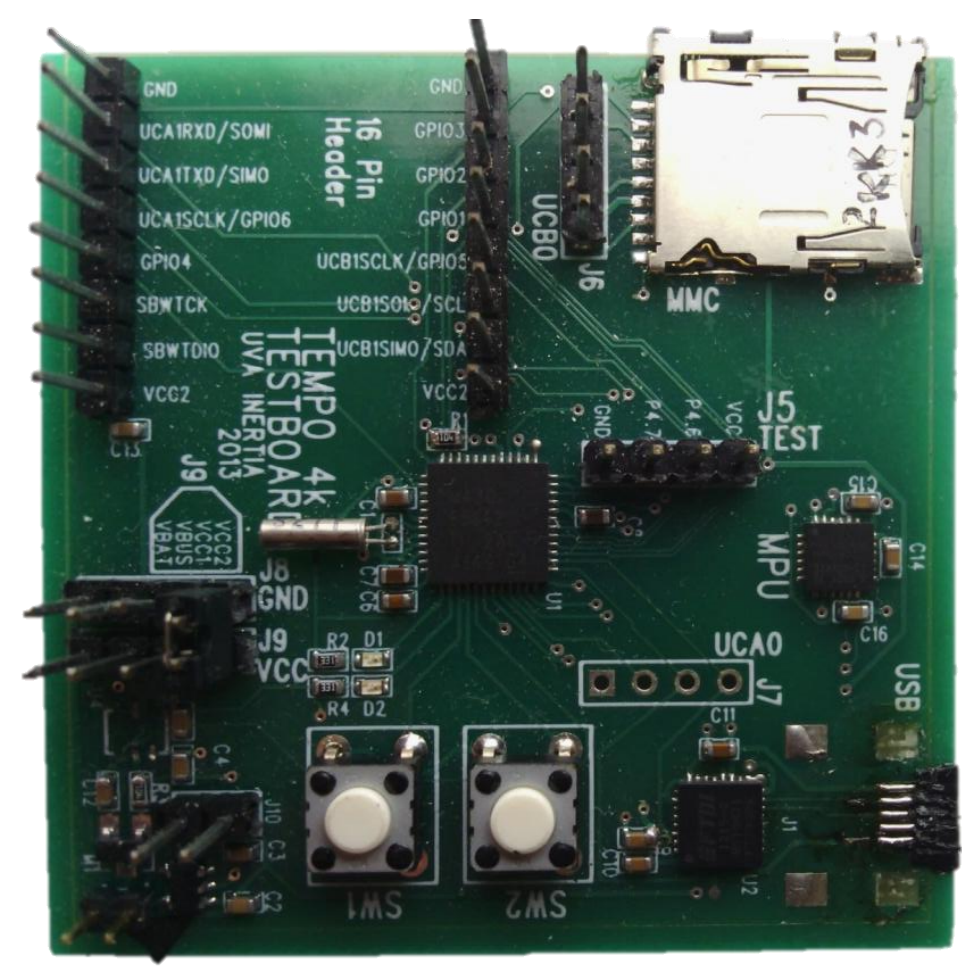

Figure 79: TEMPO 4 Test Board Populated Hardware

\subsection{TEMPO 4 Final Hardware Platform}

Once the TEMPO 4 test board platform had been rigorously vetted and each of its subsystems deemed functional, the development of the final TEMPO 4 hardware platform began. The 1x1 inch form-factor implied the board would likely need to be 4-layers, so this was selected as the preliminary stack-up for the design. The final TEMPO 4 hardware platform design and specification is summarized in the section below.
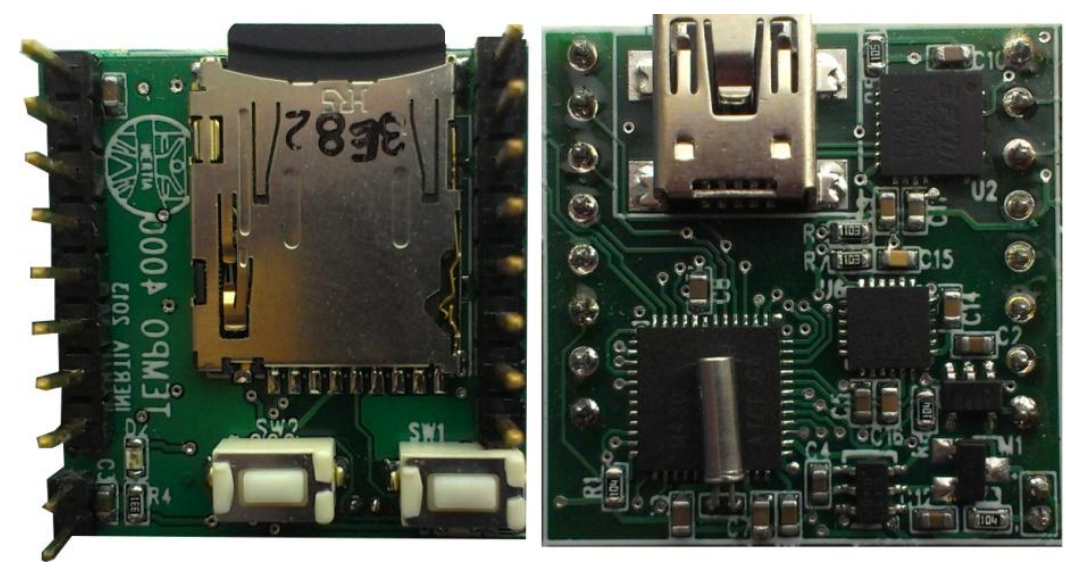

Figure 80: TEMPO 4 Final System Hardware 
The core TEMPO 4 platform supports on-board interfacing via USB, microSD, pushbutton, LEDs, and the 16-pin generic development header described earlier in this document. The system is controller via an MSP430 microcontroller and samples human motion data using an MPU6050 singlechip IMU.TEMPO 4 can operate using either rechargeable Lithium-based battery chemistries or non-rechargeable batteries of the user's choice that interface using a standard 2-pin JST connector. The entire platform fits on a 1x1 inch 4-layer PCB that can be inscribed within the previous TEMPO 3.2 system's area footprint.

Figure 81: TEMPO 4 Final Board and Previous TEMPO 3.2 The push-button switches, LEDs, battery charging circuitry, and even System Main-board stacked for Area Comparison possibly the on-board IMU can be considered do-not-populate (DNP) devices for those applications which do not demand use of these components, reducing bill of material size and cost. As previously mentioned, no custom plastic housing was created for the TEMPO 4 platform as it is understood that a variety of possible deployments may field a variety of different casing requirements.

This information, along with more specific details of operation and component location, are provided in the table and figure below.
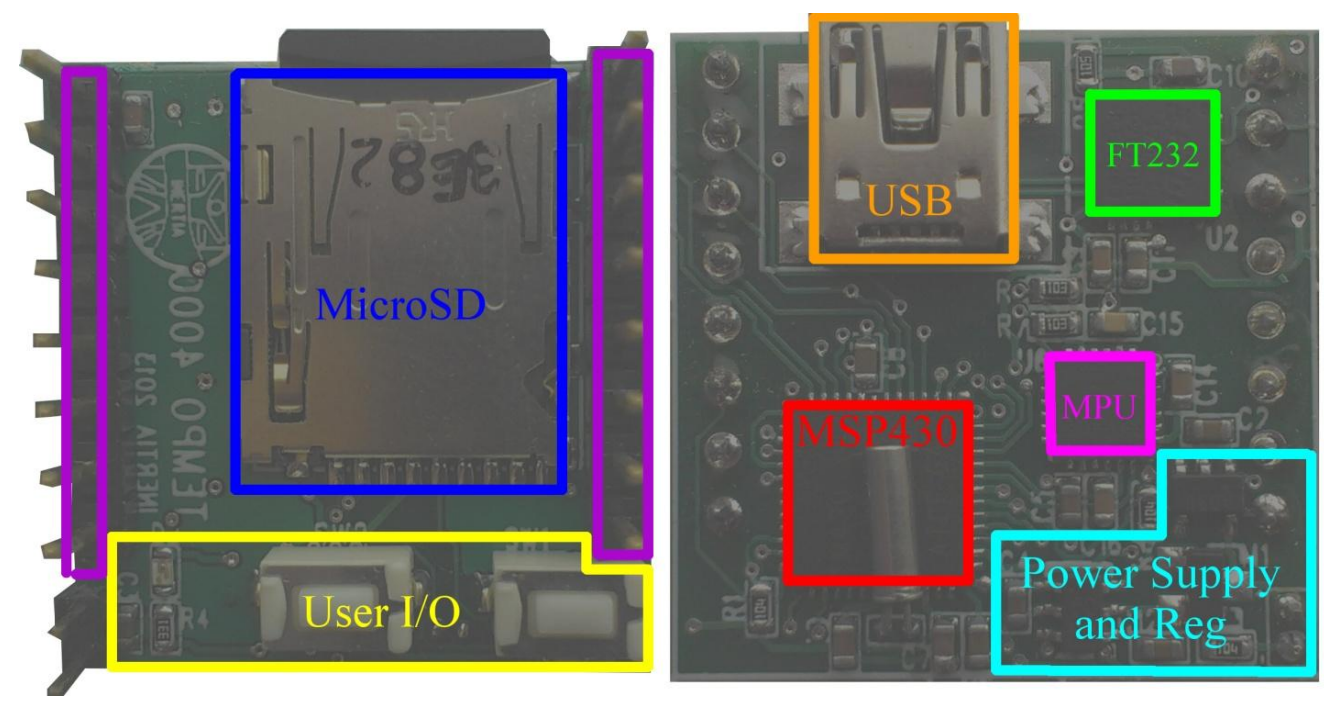

Figure 82: Documented TEMPO Hardware Layout 


\begin{tabular}{|c|c|c|}
\hline Feature & Parameter & Value \\
\hline \multirow{4}{*}{ Controller } & $\mathrm{MCU}$ & MSP430F5342 \\
\hline & Max Clock Speed & $24 \mathrm{MHz}$ \\
\hline & Active Current & $<300 \mathrm{uA} / \mathrm{MHz}$ \\
\hline & Programming Interface & Spy Bi-wire (SBW) \\
\hline \multirow{4}{*}{ On-board Sensing } & IMU Platform & $\begin{array}{l}\text { MPU6050/9150 } \\
3 \text { axis accelerometer } \\
3 \text { axis gyroscope } \\
\text { ( } 3 \text { axis magnetometer) }\end{array}$ \\
\hline & Sampling Rate & $1 \mathrm{kHz}$ \\
\hline & Output Sample Resolution & 15 bits \\
\hline & Active Current & $\begin{array}{l}\text { 500uA (accelerometers) } \\
3.5 \mathrm{~mA} \text { (gyroscopes) } \\
\text { 350uA (magnetometer) }\end{array}$ \\
\hline \multirow{2}{*}{ USB Interface } & Delivered Current & $100 \mathrm{~mA}$ \\
\hline & Max UART Baud & 1MBaud \\
\hline \multirow{5}{*}{ Flash Storage } & Storage Format & microSD \\
\hline & Max Card Capacity & $2 \mathrm{~GB}$ \\
\hline & Card Lifetime (@ 2GB) & 15 days \\
\hline & Active Current & $100 \mathrm{~mA}$ \\
\hline & Sleep Current & $10 \mathrm{uA}$ \\
\hline \multirow{4}{*}{ Development Header } & Digital Interfaces & 1 UART/SPI, 1 I2C \\
\hline & Analog Input & 4 Channels, 12-bit SAR \\
\hline & Other Interfaces & 2-pin SBW header \\
\hline & Power Supply & $150 \mathrm{~mA} 3.3 \mathrm{~V}$ output 1 \\
\hline \multirow{3}{*}{ Power Supply } & Isolated Outputs & 2 \\
\hline & Output Voltage & $3.3 \mathrm{~V} 1$ \\
\hline & Max Output Current & $150 \mathrm{~mA}$ (per output) \\
\hline \multirow{4}{*}{ Battery Input } & Battery Connector & 2-pin JST \\
\hline & Rechargeable Chemistries & LiPo, LiIon \\
\hline & Suggested Capacity & 300-1000mAh \\
\hline & Battery Lifetime (@300mAh) & $30-60$ hours \\
\hline \multirow{4}{*}{ Form Factor } & Size & $25.4 \times 25.4 \mathrm{~mm}$ \\
\hline & Mass w/o battery & $7 \mathrm{~g}$ (w/o battery) \\
\hline & Mass w/ 400mAh battery & $16 \mathrm{~g}$ \\
\hline & Mass w/ 850mAh battery & $24 g$ \\
\hline
\end{tabular}

Ahead of final design profiling, power and functionality classification of all the subsystems described above allowed for accurate modeling of expected overall system power during their interaction. A combination of device data sheet information and results of in-lab measurements were then used to produce a system-level power budget model for the TEMPO 4 platform. This was useful in determining approximate system power and affiliated lifetime for a given battery capacity and firmware use case. To demonstrate the significance of the impact of application changes on node operation, approximate power budgets for the TEMPO 4 system in the 3 and 6 DoF use-cases is provided in the affiliated section of this chapter. 


\subsection{TEMPO 4 Firmware Control Structures}

The TEMPO 4 firmware contributions will be summarized in two sections as part of this system-level analysis: libraries for future system development and fixed structure implemented for the purpose of emulating the previous TEMPO platform's 6 DoF IMU collection use-case.

\subsubsection{TEMPO 4 Firmware Libraries}

The primary TEMPO 4 firmware contribution is a rigorously tested and iteratively developed set of C libraries for the MSP430 with Doxygen-based inline comments for HTML documentation generation. The set of base-level system functionality contained in the libraries is varied and diverse. For this reason, some of the most crucial contribution of this work is summarized in the sub-section below and in addition the firmware itself is provided in Appendix B of this work for more careful review.

\section{Clocking and Time Management}

Routines for configuration and maintainance of the on-chip Digitally Controlled Oscillator (DCO) make use of a runtime, code-independent FLL circuit provided in the clock management module. By referencing this FLL from an off-chip oscillator and allowing it, instead of the user, to continuously adapt the DCO control bits, a stable average system clock rate can be achieved. Library support for on-chip clocking structures includes DCO and FLL initialization routines, along with macro-based system clocking definitions to allow for references to the main system clock rate throughout user code.

In addition to system clock control, the time management code also provides setup and sampling routines for the on-chip RTC and a convenient time structure for pointer-based retrieval of system time values. As part of work not described in this document a hardware-timing library, used for accurate run-length profiling during real-time code execution, was also created for the MSP430's timer peripheral.

\section{Communications and Interfacing}

The TEMPO 4 communications library has been described at great length throughout this work as it is considered one of the key enabling contributions to system operation. Implementing an easy-to-use onetime configuration registration function, the communication library makes use of socket-style interfaces to implement UART, SPI, or I2C serial data protocols in the on-chip USCI modules of the MSP430. This communications code falls at the heart of USB, MMC, and IMU communication for the TEMPO 4 platform.

The USB communication library is a thin wrapper for the underlying UART communications code, as this lower-level piece of code captures all functionality required for interfacing the simple 2-wire UART 
on the FT232 transceiver IC. MMC libraries were ported from TEMPO 3.2 to replace the older, dedicated, blocking SPI communication functions with the newer, interrupt-driven communications code. Last, but not least, the MPU6050 driver containing the device register map and simplified control API was created on top of two basic $\mathrm{I} 2 \mathrm{C}$ read and write functions provided as the interface to this portion of the communications library.

In addition to this communications code comes a TEMPO-specific HAL file intended to make interfacing the on-board user I/O and control signals relatively easy by offering up simple macro-based functionality for changing, reading, and configuring digital pin states for use during firmware operation. An interruptmasking library is also provided for the registration of callback functions for common asynchronous signals of interest, such as the IMU data ready line, or user push-button status.

\section{Command Interface and File System}

The TEMPO 4 command interface is written on top of the aforementioned FT232 USB driver code produced using the communications library. It closely mimics the previous TEMPO 3.2F platform's command interface with improved throughput and offload-time ratios as a result of higher baud rates and decreased command lengths and delays. The decision to produce a command interface so similar to that of the 3.2 node was largely motivated by its successful use with the previous system and the existence of Python 3.3 libraries already capable of interfacing this custom communication standard.

The TEMPO 4 file system implements a sequentially written, linked-list structure for low-complexity data logging. By eliminating the more complex file system structure implemented in the TEMPO 3.2 node this work seeks to both improve file system reliability and reduce file system overhead, while simultaneously providing better wear-leveling in the flash storage.

\subsubsection{Top-level System Operation}

For the sake of comparative power measurements, and demonstration of the added value of this work to the TEMPO platform's battery lifetime and form-factor constraints a top-level firmware operating model similar to that of the previous TEMPO 3 system is adopted. As previously referenced, this firmware model makes use of first-in-first-out (FIFO), event-queue driven operation to coordinate system operation in the presence of both asynchronous interrupts and user-defined, synchronous code routines. In addition to the event-queue described previously, the TEMPO 4 platform also uses a simple state-machine to control system operation and modify interface behavior based around top-level system state. This approach is borrowed from the TEMPO 3.2 core operating firmware, which used a similar state-machine 
model to interpret system-level events and coordinate device operation without the presence of a command-driven Bluetooth interface.

Essentially, the system has only 3 primary operating modes: command, sleep, and collection. Transitions between these three operating modes are based primarily upon two system-level control signals: the hardware USB connection indicator and the firmware data-collection enabled (DCE) flag, set by the pushbuttons or command interface. In addition to these three basic states, three additional states are introduced to guard specific execution against state-coordinated intervention, these are start and end session, along with idle. Start and end session imply the node is performing affiliated sensor configuration and file system operations, and exist to prevent sampling or collection runtime routines from interfering with this effort. The idle state represents a method by which system operation can be temporarily suspended during collection without the low-power reconfiguration of the system hardware for sleep. This state is also used to protect any code dedicated to running at the end of an entire session, for example on-node extraction of key metrics from a long period of stored session data.

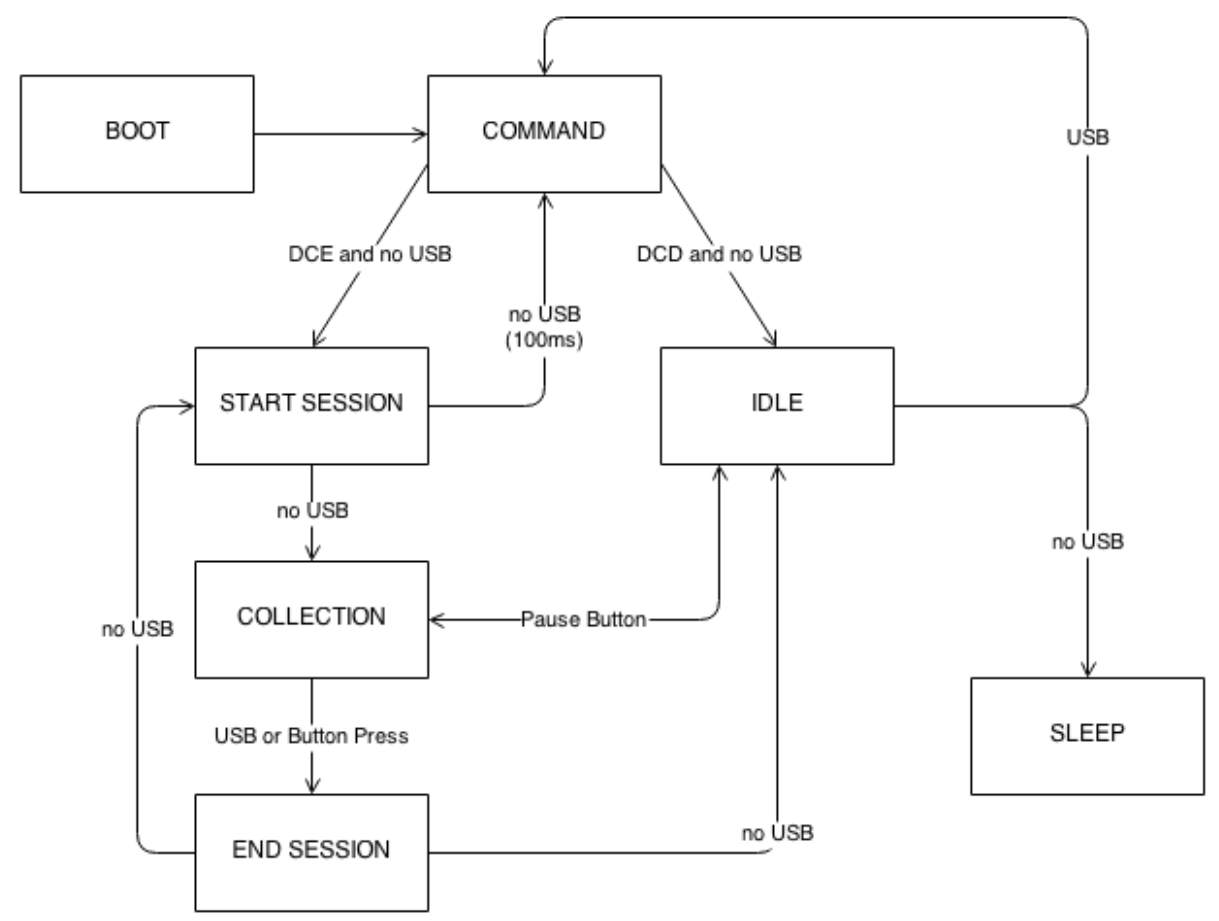

Figure 83: TEMPO 4 Firmware State Machine

Using the method of operation described in this sub-section a minimal functionality, 6 DoF IMU use case was drafted for power measurement on the TEMPO 4 system. Evaluation of the platform-level power budget of the TEMPO 4 system using this early-stage firmware operating model is provided in the following section. 


\subsection{TEMPO 4 Power Budget Analysis}

This section proposes two evaluations of the TEMPO 4 system for consideration before adoption of the platform into any new sensing deployment. The first portion of this section contains information regarding TEMPO 4's performance in the 3 and 6 DoF use-cases, while the second details modeling efforts affiliated with producing approximate TEMPO battery lifetime for a wide variety of possible use cases.

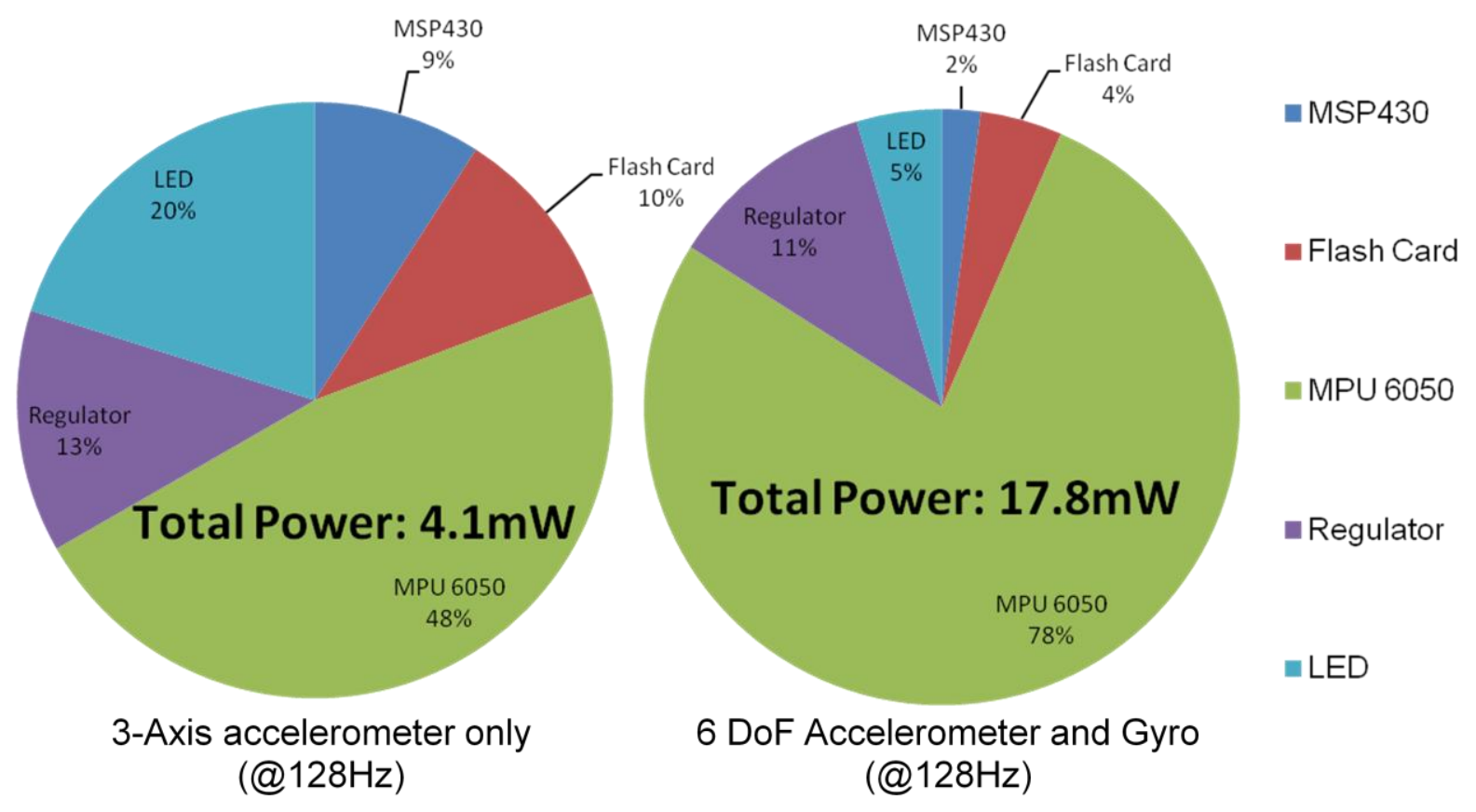

Figure 84: Approximate Power Budget for TEMPO 4 in the 3 and 6 DoF Use Cases

As can be seen in Figure 84 above, both the relative and absolute magnitudes of the TEMPO 4 system components can vary widely, even over potentially similar use cases. In the 3 DoF, accelerometer only, use-case the node does save on IMU costs by power gating the expensive gyros, but also through reduced flash card usage due to decreased output sensor data rate. For the sake of this figure it is assumed that the LED blinks during collection, staying on for $0.1 \mathrm{~s}$ two times a second, to indicate continued data collection. It can be seen that in the power budget at left, this LED operation comprises a significant portion of the overall system power consumption in the $3 \mathrm{DoF}$ use case, at about $1 / 5$ of the total power.

As previously mentioned, before final integration and platform measurement, the TEMPO 4 system was modeled using a weighted-sum approach to produce an accurate approximation of overall system energy from measurements of each subsystem during device operation. 
Since the USB transceiver used as part of this work is in a self-powered configuration, supplied from the bus itself, its power overheads are not included in this system analysis. Flash and sensor system are modeled based around their average power per-use and scaled through data source or sink rates. LED power is modeled using two simple parameters: on and off time in a per/second context. MCU power is determined in a slightly more complex manor, using core voltage, operating frequency, and an activity factor indicating the portion of time spent in active mode. Finally regulator overhead is calculated based upon the provided system input voltage, and power efficiency implied by the regulator forward voltage drop.
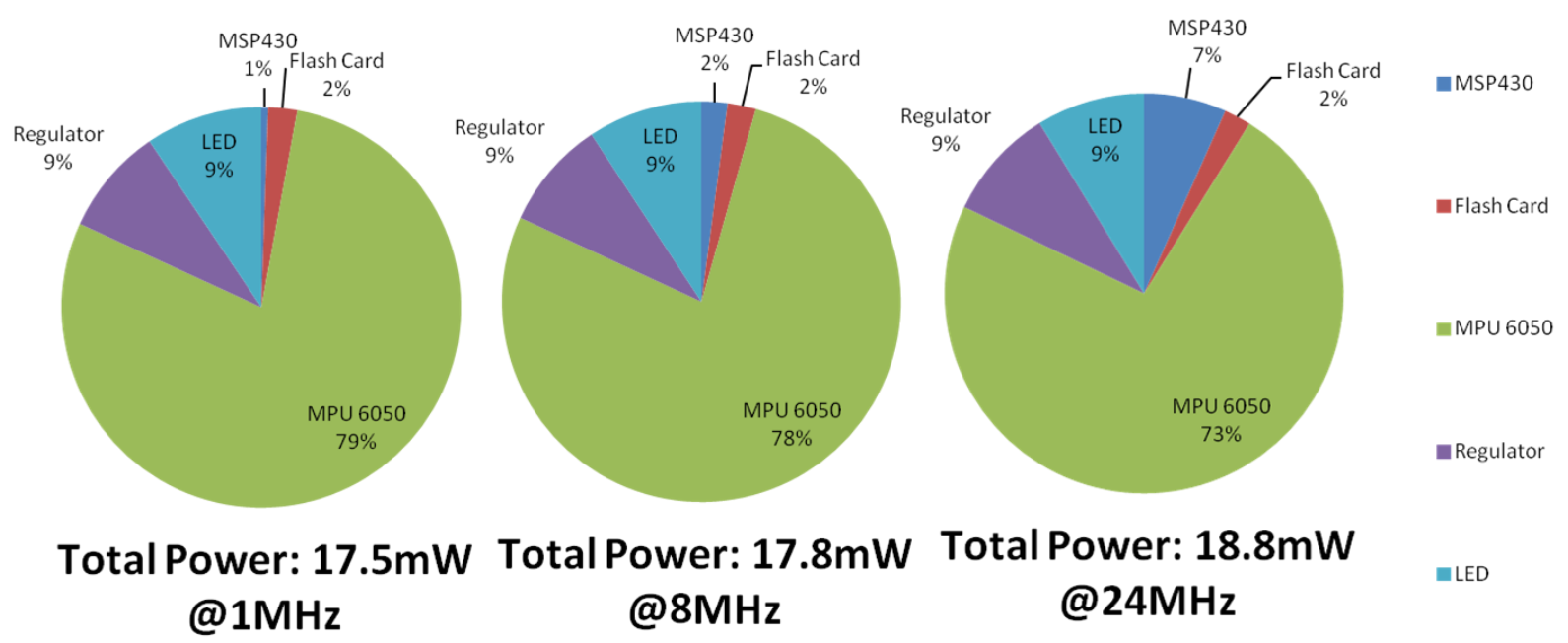

ELED

Figure 85: Predicted TEMPO 4 System Power Budget at Various Operating Frequencies

As a result of this easy-to-use device power model, the impact of altering various operating parameters in the presence of a number of source and sink conditions is observable directly. In an attempt to demonstrate the usefulness of this capability the TEMPO 4 projected system power budget over a variety of operating frequencies is shown in Figure 85. The key conclusion from power-budget profiling conducted to this end, was that operating in the sub- $8 \mathrm{MHz}$ region, where the lowest core voltage levels are possible, demonstrates the best implications on percentage of system power-budget consumed by the MSP430, with little to no additional benefit offered in reduction of overall system power budget available below this operating frequency. This is largely a result of the energy equality implied by the trade-off of running at higher power/frequency for less time or a lower power/frequency for greater time.

In addition to modeling various core operating conditions, this model is also capable of attempting to predict system power usage during the diverse data collections implied throughout this work. In order to enable future modeling efforts, the top-level system model provides parametrized MMC power estimation based on access rate, in writes per second, and communication frequency (in MHz). In addition the core 
and regulator overheads are also determined using parametrized representations to allow for extensions of the model to future use cases. If a new sensor or control strategy is to be modeled, the level of depth of this model is left to the future designer. For the case of the MPU6050 an always-on operating model was assumed to provide an upper-bound on IMU power consumption; however, if a more efficient MPU control sheme is implemented in the future, it may yield significant benefits over this simplistic alwayson model. To demonstrate the efficiacy of such solutions it is recommended that future platform modeling efforts use a more complex IMU model to precisely determine power savings offered up by this sort of sensor duty-cycled operation. 


\section{Chapter 8}

\section{Conclusions and Future Directions}

The design of the TEMPO 4 platform provides an interesting case study for the importance of the concepts of co-design and subsystem oriented, iterative development and testing in the success of any tightly-integrated, ultra-low power sensor system. The relevant concepts of power delivery, system control, sensor acquisition, and data reporting are all addressed as part of this work, giving thorough consideration to the desired metrics of form-factor, lifetime, ease-of-interfacing, reliability, and flexibility. The solution arrived at succeeds largely at accomplishing the established goals of this work, and provides

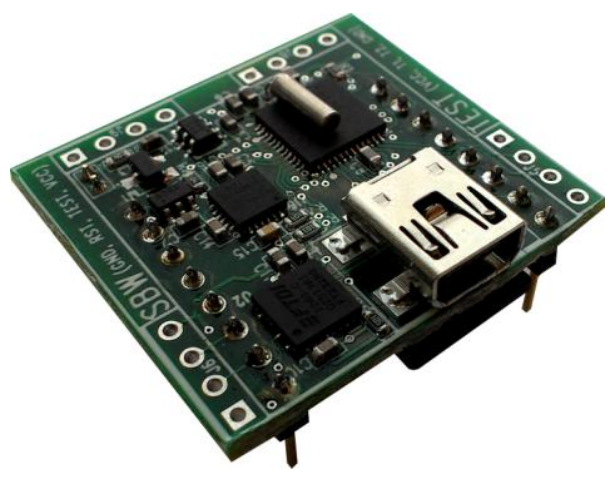

Figure 86: Final TEMPO 4 Platform an open, low-power, wearable, hardware-firmware platform on which developers can rapidly prototype for research applications of their choosing.

The significant conclusions arrived at throughout the process of the TEMPO 4 design process are those of the importance of hardware, firmware, and application layer co-design and iterative, vertically-integrated hardware-firmware development. By producing multiple hardware platforms, each designed to test and measure the functionality of small hardware subsystems, rather than the entire platform, during operation both increased reliability and flexibility of all sub-circuits is assured. As an added benefit, this subsystem oriented development process allows for convenient modeling of system-level performance based upon mathematical extrapolation of parameters measured on the test bench. Allowing future developers to profile the impact of adding new sensing and reporting modalities to the existing node's piecewise power budget.

\subsection{TEMPO 4 Design Conclusions}

In regard to battery chemistry it is concluded that the impact of energy storage mass and volume on formfactor must be carefully considered on an application-by-application basis before selection of any specific chemistry or cell size. Though most commercially available batteries perform with similar energy-density characteristics, reduced form-factor constraints, specifically that of mass, along with the popularity of rechargeable cells promotes the use of Lithium chemistries in the wearable design space. For this reason, 
the TEMPO 4 platform supports an optional Lithium Polymer and Ion battery charging circuit and standard JST connector with reverse polarity protection.

Ultra-low power regulation is a challenge as the operating overheads implied by switched-mode regulation out-weight the efficiency benefits it produces as forward current decreases. Since the TEMPO 4 platform uses a system-wide operating voltage of 3.3V and Lithium cells produce a nominal output of just 3.6V, LDO linear regulation is considered for use. The result of the selection of this linear regulator topology is both higher regulation efficiency and lower area overheads dedicated to system regulation.

The controller selection process demonstrates that low-power MCUs are predominate controller topologies to consider for use in the ULP body-worn context. Through extensive surveys of available hardware and affiliated programming and tool chain support it is determined the MSP430 best fits the needs and speficifications of the TEMPO 4 platform, based on form-factor and serial interfacing constraints. The availability and use of common serial protocols throughout the TEMPO development process is of utmost importance. By iteratively developing and testing a set of vertically-integrated firmware libraries for coordinating hardware communication modules and low-level peripheral management, a reliable base on which to build additional platform drivers is established.

In order to demonstrate the flexibility and robustness of these underlying libraries USB translation, MMC communication, and sensor sampling drivers are all built atop of this basic, lower-level communications functionality. In addition to this basic driver code, an example operating system, with event queue-driven execution and a small finite state machine, is used to coordinate calls between these driver routines to demonstrate system operation. Throughout the process of this demonstration, the impact of communication rates, system power consumption, and effective sleep management are acknowledged and addressed.

It is anticipated that using this top-level example configuration, the TEMPO 4 platform will be able to collect data for at least 2 days continuously in the flash collection use-case. In addition, the overall size and mass improvements relative to the previous TEMPO 3 platform are near 50\%. This means the TEMPO 4 platform provides nearly identical performance, with $4 \mathrm{X}$ benefits in lifetime, $2 \mathrm{X}$ benefits in form-factor, and vastly increased ease-of-interfacing, reliability, and flexibility. This improvement is accounted for in part by more efficient, tightly integrated firmware control and also in part by progression in state-of-the-art commercial platforms, primarily IMUs. 


\subsection{TEMPO 4 Future Directions}

Though just completed it is possible that the TEMPO 4 hardware platform could already benefit from a minor hardware revision. By implementing an FT230x serial USB to UART translator IC instead of the FT232 used in this work, a transceiver IC area reduction of about $30 \%(5 \times 5 \mathrm{~mm}$ to $4 \times 4 \mathrm{~mm})$ is possible. Similarly, by moving the on-board IMU from the MPU6050 or 9150 IC's 4x4mm footprint to the newer MPU9250 3x3mm footprint, this work could save up to $20 \%$ in overall IMU area. In addition it is recommended a better solution for protecting the battery management IC against reverse voltage polarity is implemented, as well as a possible re-design of the user push-buttons to implement a lower-profile, and lower cost alternative. Last but not least, it is recommended that a unique casing for the hardware platform and a widely available lithium battery is produced, preferably in an easily $3 \mathrm{D}$ printable format for open source distribution.

While this work does propose a convenient, event-queue driven operating model for the purpose of system measurement and comparable evaluation with the TEMPO 3.2 platform, further firmware exploration is definitely suggested for the TEMPO 4 host controller. In regard to system operation, the porting of open libraries such as TI's FAT16 storage management or SimpliciTI radio code for the CC11xx to run on top of the TEMPO 4 communications library is an interesting option. Data processing libraries implementing more complex on-node feature extraction or filtering could also be ported from almost any code previously created for the MSP430 ISA or similar 16-bit architecture. Last, but not least, in regard to firmware improvements, the sequentially-written file system implemented as a part of this work may hold some promise for use in a radio interface as well with minimal modifications. Since the output of this file system management is always a sequentially written, CRC validated stream of bytes, it lends itself well to reliable transmission through the noisy wireless channel with built-in data validation and fixed-length metadata structures.

In addition to providing more commonly accepted protocols for user interfacing and rigorously tested operating models, a number of embedded operating systems provide features like platform debug interfaces and pre-compiled code libraries to preserve and protect the user code space while allowing access from the development environment. The increased MCU processing capabilities of the MSP430 selected for use in this design are intended to allow for flexibility to adapt to the constraints of most existing operating systems for the MSP430 platform. While a number of embedded operating systems including Contiki, FreeRTOS, TinyOS, and TI's SYS/BIOS are all available for the MSP430 platform, the co-design focus of this work dissuades the use of generalized operating system models in favor of lower overhead application-specific top-level firmware organization. Future investigations might propose 
an ultra-low overhead RTOS for the wearable sensor node design space, configured to function effectively using the low clock speeds, and intimate level of peripheral management often required for these ULP deployments.

While it is acknowledged that increased complexity of firmware control and device configurations may mean additional system-level modeling challenges, it is also recommended that the TEMPO 4 platform power model is updated to support more generic on-node sampling and processing efforts. By providing more accurate, parametrized models of on-chip peripherals and IMU operation, a broader, more generalized tool for use in developing system power budgets for new deployments could be enabled. Though the power modeling conducted for the sake of this work is considered rather complete for the purpose of developing top-level 3, 6, and 9 DoF inertial motion collection power budgets, it could also be improved to better capture IMU and reguatlor non-idealities and provide a more accurate measurement of true system performance as well.

In regard to software support and offload interfaces, as previously described, the TEMPO 4 node uses the same FT232 USB-to-UART translator as its predecessor along with a similar command interface. This allows for use of previously created Python libraries for communicating with the device over USB, supporting successful command exchanges all the way to $1 \mathrm{Mbaud}$. In regard to future directions, it is first and foremost recommended this Python communication interface is wrapped in additional GUI-based code for user-side data offload. As demonstrated in the case of the TEMPO 3.2 platform, a good hardware and firmware design mean relatively little without an intuitive end-user interface, and for this reason a possible integration of the TEMPO 4 communication class into the BodyDATA software framework, created as part of another INERTIA student's master's thesis work, is considered.

In addition to BodyDATA oriented support, it is also suggested that a PC-side, rapid offload interface, oriented around the Disk Dump (DD) command and full-speed, parallel MMC offload technique is implemented to dump the unformatted contents of the TEMPO 4 node's microSD card to a file on disk. A more efficient, natively compiled PC-side program could then parse this dumped data and interpret any and all valid sectors in incredibly little time comapared to serialized offload approaches. This high-speed offload approach is recommended for forensic investigation of cards prone to sporadic failure. 


\section{References}

[1] Barth, A.T.; Hanson, M.A.; Powell, H.C.; Lach, J., "TEMPO 3.1: A Body Area Sensor Network Platform for Continuous Movement Assessment," Wearable and Implantable Body Sensor Networks, 2009. BSN 2009. Sixth International Workshop on, vol., no., pp.71,76, 3-5 June 2009

[2] Kumar, P.; Pandey, P.C., "A wearable inertial sensing device for fall detection and motion tracking," India Conference (INDICON), 2013 Annual IEEE , vol., no., pp.1,6, 13-15 Dec. 2013

[3] FitBit, (2014). "FitBit Zip Specs", FitBit Zip [Online]. Available: http://www.fitbit.com/zip/specs

[4] X-IO Technologies, (2012). “x-IMU”, X-IO Products [Online]. Available: http://www.xio.co.uk/products/x-imu/

[5] Shimmer, (2014). "Shimmer 3", Shimmer Shop [Online]. Available: http://www.shimmersensing.com/shop/shimmer3

[6] Q. Li, J.A. Stankovic, M. Hanson, A. Barth, J. Lach, “Accurate, Fast Fall Detection Using Gyroscopes and Accelerometer-Derived Posture Information,” International Conference on Body Sensor Networks, 138-43, 2009

[7] M.A. Hanson, H.C. Powell Jr., A.T. Barth, J. Lach, M. Brandt-Pearce, "Neural Network Gait Classification for On-Body Inertial Sensors," International Conference on Body Sensor Networks, 181-6, 2009

[8] A.T. Barth, B.C. Bennett, B. Boudaoud, J.S. Brantley, S. Chen, C.L. Cunningham, T. Kim, H.C. Powell, Jr., S.A. Ridenour, J. Lach, "Longitudinal High-Fidelity Gait Analysis with Wireless Inertial Body Sensors,” Wireless Health, 192-3, 2010

[9] H.C. Powell Jr., M.A. Hanson, J. Lach, “On-Body Inertial Sensing and Signal Processing for Clinical Assessment of Tremor," IEEE Transactions on Biomedical Circuits and Systems, 3(2):108-16, April 2009

[10] A. Bankole, M. Anderson, T. Smith-Jackson, A. Knight, K. Oh, J.S. Brantley, A.T. Barth, J. Lach, "Validation of Non-Invasive Body Sensor Network Technology in the Detection of Agitation in Dementia," American Journal of Alzheimer's Disease \& Other Dementias, 27(5):346-354, August 2012

[11] Ojetola, O.; Gaura, E.I.; Brusey, J., "Fall Detection with Wearable Sensors--Safe (Smart Fall Detection)," Intelligent Environments (IE), 2011 7th International Conference on , vol., no., pp.318,321, 25-28 July 2011 
[12] Woon-Sung Baek; Dong-Min Kim; Bashir, F.; Jae-Young Pyun, "Real life applicable fall detection system based on wireless body area network," Consumer Communications and Networking Conference (CCNC), 2013 IEEE , vol., no., pp.62,67, 11-14 Jan. 2013

[13] Martin, E., "Real time patient's gait monitoring through wireless accelerometers with the wavelet transform," Biomedical Wireless Technologies, Networks, and Sensing Systems (BioWireleSS), 2011 IEEE Topical Conference on , vol., no., pp.23,26, 16-19 Jan. 2011

[14] Schulze, M.; Calliess, T.; Gietzelt, M.; Wolf, K. H.; Liu, T. H.; Seehaus, F.; Bocklage, R.; Windhagen, H.; Marschollek, M., "Development and clinical validation of an unobtrusive ambulatory knee function monitoring system with inertial 9DoF sensors," Engineering in Medicine and Biology Society (EMBC), 2012 Annual International Conference of the IEEE , vol., no., pp.1968,1971, Aug. 28 2012-Sept. 12012

[15] Pendharkar, G.; Percival, P.; Morgan, D., "Evaluating bouncy gait in idiopathic toe-walkers using accelerometer," Intelligent Sensors, Sensor Networks and Information Processing, 2008. ISSNIP 2008. International Conference on , vol., no., pp.331,334, 15-18 Dec. 2008

[16] Yoneyama, M.; Kurihara, Y.; Watanabe, K.; Mitoma, H., "Accelerometry-Based Gait Analysis and Its Application to Parkinson's Disease Assessment-Part 2: A New Measure for Quantifying Walking Behavior," Neural Systems and Rehabilitation Engineering, IEEE Transactions on , vol.21, no.6, pp.999,1005, Nov. 2013

[17] Barth, Jens; Klucken, Jochen; Kugler, Patrick; Kammerer, Thomas; Steidl, Ralph; Winkler, Jurgen; Hornegger, Joachim; Eskofier, Bjorn, "Biometric and mobile gait analysis for early diagnosis and therapy monitoring in Parkinson's disease," Engineering in Medicine and Biology Society,EMBC, 2011 Annual International Conference of the IEEE , vol., no., pp.868,871, Aug. 30 2011-Sept. 32011

[18] Gupta, P.; Dallas, T., "Feature Selection and Activity Recognition System Using a Single Triaxial Accelerometer," Biomedical Engineering, IEEE Transactions on , vol.61, no.6, pp.1780,1786, June 2014

[19] YEI Technologies, (2014). “3-Space Micro USB,” YEI Products [Online]. Available: http://www.yeitechnology.com/productdisplay/3-space-micro-usb

[20] Life Performance Research, (2013). “LPMS-B: 9-Axis IMU/AHRS/Motion Sensor with Bluetooth (wireless) Connectivity", LPMS Products [Online]. Available: http://www.lpresearch.com/9-axis-imu-with-bluetooth-wireless-connectivity/ 
[21] Actigraph, (2014). “wGT3X-BT Monitor”, Actigraph Products [Online]. Available: http://www.actigraphcorp.com/products/wgt3x-bt-monitor/

[22] FitBit (2014). "FitBit One Specs”, FitBit One [Online]. Available: http://www.fitbit.com/one/specs

[23] FitBit (2014). "FitBit Flex Specs", FitBit Flex [Online]. Available: http://www.fitbit.com/flex/specs

[24] Jawbone (2014). “Jawbone Up Tech Specs”, Jawbone Up [Online]. Available: http://jawbone.com/store/buy/up24\#tech-specs

[25] Nisarga, B.; Quiring, K.; Taylor, L (2011, April 6). “The Ultra-Low-Power USB Revolution” DigiKey Article Library [Online]. Available: http://www.digikey.com/en/articles/techzone/2011/apr/the-ultra-low-power-usb-revolution

[26] FTDI (2014). "FT232R - USB UART IC", FTDI USB Products [Online]. Available: http://www.ftdichip.com/Products/ICs/FT232R.htm

[27] FTDI (2014.) “Development Modules”, FTDI Module Products [Online]. Available: http://www.ftdichip.com/Products/Modules/DevelopmentModules.htm

[28] Jim.sh (2013). "USB to serial breakout”, MicroFTX [Online]. Available: https://jim.sh/ftx/

[29] Hanson, S.; Mingoo Seok; Yu-Shiang Lin; Zhiyoong Foo; Daeyeon Kim; Yoonmyung Lee; Liu, N.; Sylvester, D; Blaauw, D, "A Low-Voltage Processor for Sensing Applications With Picowatt Standby Mode," Solid-State Circuits, IEEE Journal of , vol.44, no.4, pp.1145,1155, April 2009

[30] SparkFun (2014). "Polymer Lithium Ion Battery - 2000mAh", SparkFun Shop [Online]. Available: https://www.sparkfun.com/products/8483

[31] Panasonic (2010, February). "NCR18650”, NNP Series [Online]. Available: http://industrial.panasonic.com/www-data/pdf2/ACA4000/ACA4000CE240.pdf

[32] Mcintosh, D.; Mars, P., "Using a Supercapacitor to Power Wireless Nodes from a Low Power Source such as a 3V Button Battery," Information Technology: New Generations, 2009. ITNG '09. Sixth International Conference on, vol., no., pp.69,78, 27-29 April 2009

[33] SparkFun (2014). "Power Connectors" Connector Basics [Online]. Available: https://learn.sparkfun.com/tutorials/connector-basics/power-connectors

[34] SparkFun (2014). "Lithium Ion/Polymer USB Battery Charger IC - MAX1555” SMD IC Products [Online]. Available: https://www.sparkfun.com/products/674

[35] Dostal, F. "Buck-boost Topology Delivers Negative Output from Positive Input" EE Times Design How-To [Online]. Available: http://www.eetimes.com/document.asp?doc_id=1273276 [36] OpenCores (2014). “OpenCores” [Online]. Available: http://opencores.org/ 
[37] Xilinx (2014). “Zynq-7000 Silicon Devices” Programmable SoC Products [Online]. Available: http://www.xilinx.com/products/silicon-devices/soc/zynq-7000/silicon-devices/index.htm

[38] Cypress (2014). "Programmable System-on-Chip" Products [Online]. Available: http://www.cypress.com/psoc/

[39] Si Labs (2014). "Patented Dual-Crossbar Architecture" SiLabs MCU Products [Online]. Available: http://www.silabs.com/products/mcu/Pages/crossbar-architecture.aspx

[40] Texas Instruments (2014). “Code Composer Studio (CCS) Integrated Development Enviroment (IDE)" TI Development Environments [Online]. Available: http://www.ti.com/tool/ccstudio

[41] Texas Instruments (2014). “MSP430F5342” MSP430 Microcontrollers [Online]. Available: http://www.ti.com/product/msp430f5342

[42] Olimex (2014). "MSP430-5510STK" Olimex MSP430 Development Boards [Online]. Available: https://www.olimex.com/Products/MSP430/Starter/MSP430-5510STK/

[43] Texas Instruments (2014). "MSP430F534x 48-Pin Target board only” [Online]. Available: http://www.ti.com/tool/msp-ts430rgz48b

[44] Texas Instruments (2010, July). "MSP430 Programming Via the JTAG Interface User's Guide" [Online]. Available: http://www.ti.com/lit/ug/slau320n/slau320n.pdf

[45] Texas Instruments (2014). "MSP-FET430UIF - MSP430 USB Debugging Interface" TI eStore [Online]. Available: https://estore.ti.com/MSP-FET430UIF-MSP430-USB-Debugging-InterfaceP616.aspx

[46] itOpen (2013, March 1). "MSP430 LaunchPad Energia Development on Linux" [Online]. Available: http://www.itopen.it/2013/03/01/msp430-energia-on-linux/

[47] ST Microelectronics (2014). "INEMO-M1” INEMO Inertial Modules [Online]. Available: http://www.st.com/web/catalog/sense_power/FM89/SC1448/PF253162

[48] ST Microelectronics (2013). "LSM330 iNEMO inertial module” [Online]. Available: http://www.st.com/st-webui/static/active/en/resource/technical/document/datasheet/DM00059856.pdf

[49] Invensense (2014). "MPU-9250 Nine-Axis (Gyro + Accelerometer + Compass) MEMS MotionTracking Device" [Online]. Available: http://www.invensense.com/mems/gyro/mpu9250.html

[50] Invensense (2014). "MPU-6050 Six-Axis (Gyro + Accelerometer) MEMS MotionTracking Device" [Online]. Available: http://www.invensense.com/mems/gyro/mpu6050.html [51] Invensense (2014). "MPU-9150 Nine-Axis (Gyro + Accelerometer + Compass) MEMS MotionTracking Device" [Online]. Available: http://www.invensense.com/mems/gyro/mpu9150.html 
[52] SparkFun (2014). “Triple Axis Accelerometer and Gyro Breakout - MPU6050” [Online]. Available: https://www.sparkfun.com/products/11028

[53] Meirowsky, S (2013, January 17). "MMC-SD-miniSD-microSD-Color-Numbers-Names" [Online]. Available: http://elasticsheep.com/wp-content/uploads/2010/01/sd-card-pinout.png

[54] "SD Card Pinout" [Online]. Available: http://elasticsheep.com/wp-content/uploads/2010/01/sdcard-pinout.png

[55] ELM (2013, February 18). "How to Use MMC/SDC" [Online]. Available: http://elmchan.org/docs/mmc/mmc_e.html

[56] Foust, F. (2004). "Secure Digital Card Interface for the MSP430 Application Note" [Online]. Available: http://alumni.cs.ucr.edu/ amitra/sdcard/Additional/sdcard_appnote_foust.pdf

[57] Texas Instruments (2014). "SimpliciTI - RF Made Easy" TI Wireless Connectivity [Online]. Available: http://www.ti.com/corp/docs/landing/simpliciTI/index.htm?DCMP=hpa_rf_general

[58] Weyn, M.; Ergeerts, G.; Wante, L.; Vercauteren, C.; Hellinckx, P. (2013, July 18). "Survey of the DASH7 Alliance Protocol for 433MHz Wireless Sensor Communication” [Online]. Available: https://dash7.memberclicks.net/assets/PDF/hindawi\%20-\%20oss.pdf

[59] Z Wave (2014). “About Z-Wave”[Online]. http://www.Z-wave.com/what_is_Z-wave

[60] DASH 7 Alliance (2012). "Feature Comparison" [Online]. Available: http://www.dash7.org/feature-comparison

[61] Microchip (2014). “Embedded Bluetooth” Wireless Technology [Online]. Available: http://www.microchip.com/pagehandler/en-us/technology/bluetooth/technology/home.html

[62] Blue Radios (2013). "BR-LE4.0-S2A (CC2540) Summary Spec" Bluetooth 4.0 Single Mode Modules [Online]. Available: http://www.blueradios.com/hardware_LE4.0-S2.htm

[63] SparkFun (2014). "XBee Pro 50mW U.FL Connection - Series 2 (ZigBee Mesh)” [Online]. Available: https://www.sparkfun.com/products/10420

[64] Roving Network (2009). "WiFly GSX 802.11 b/g Wireless LAN Module" [Online]. Available: https://www.sparkfun.com/datasheets/Wireless/WiFi/rn-131-ds.pdf 


\section{Appedix A}

\section{TEMPO 4 Final Hardware Design}

This section provides a full schematic for the TEMPO 4 platform in 5 subsystems intended to make these images more viewable, and allow for better explanation of each sub-circuits contents and function.

\section{Charging and Regulation}

The MAX1555 Lithium Polymer and Ion battery management solution, along with AP7312 dual-output LDO linear regulator and reverse voltage protection circuit are provided in the schematic below.
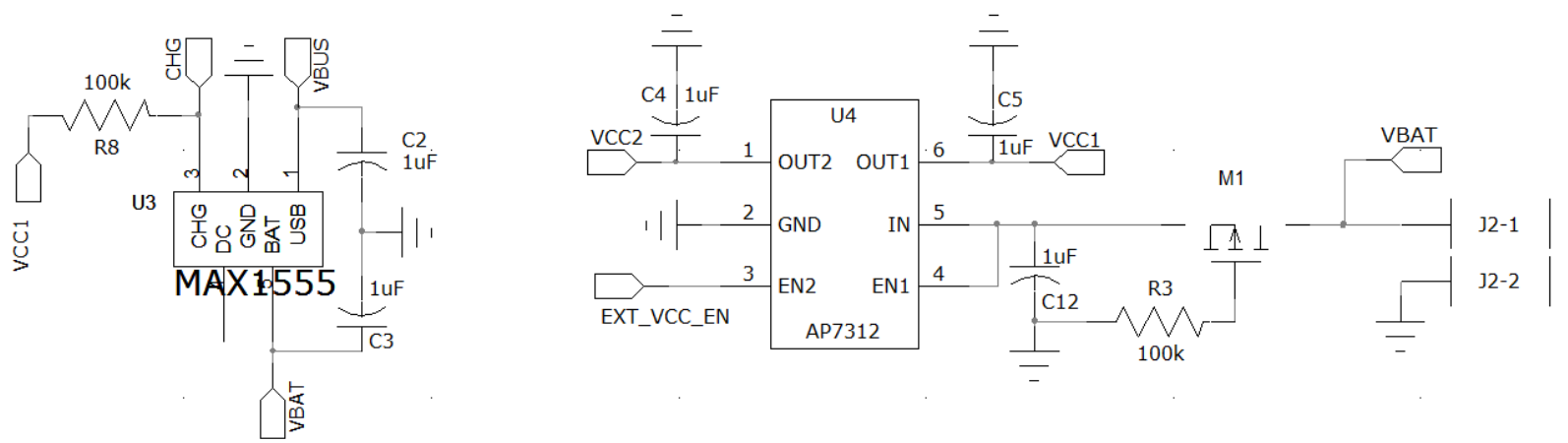

\section{USB Transceiver}

The FT232 USB-to-UART transceiver is shown below in the bus-powered configuration.

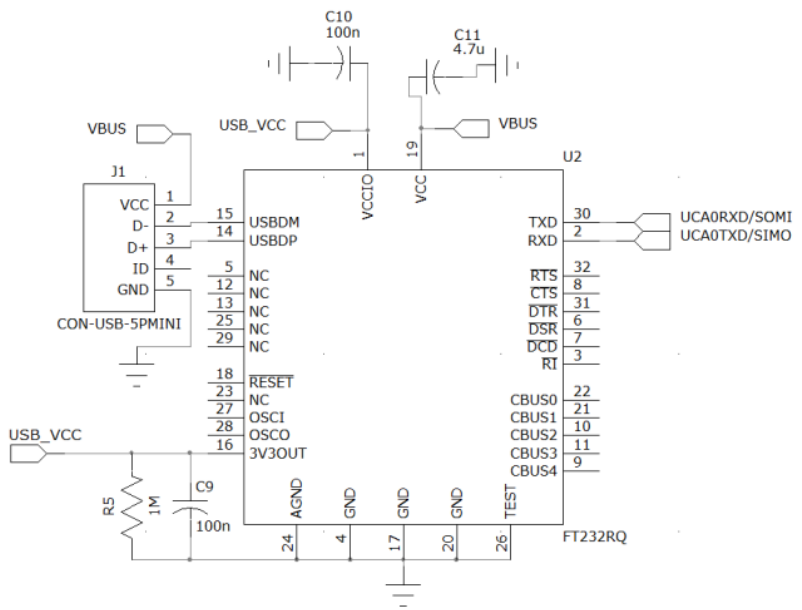




\section{IMU}

The MPU6050 IMU with its appropriate clock and data connections, decoupling capacitors, and I2C pullups is provided in the schematic below.

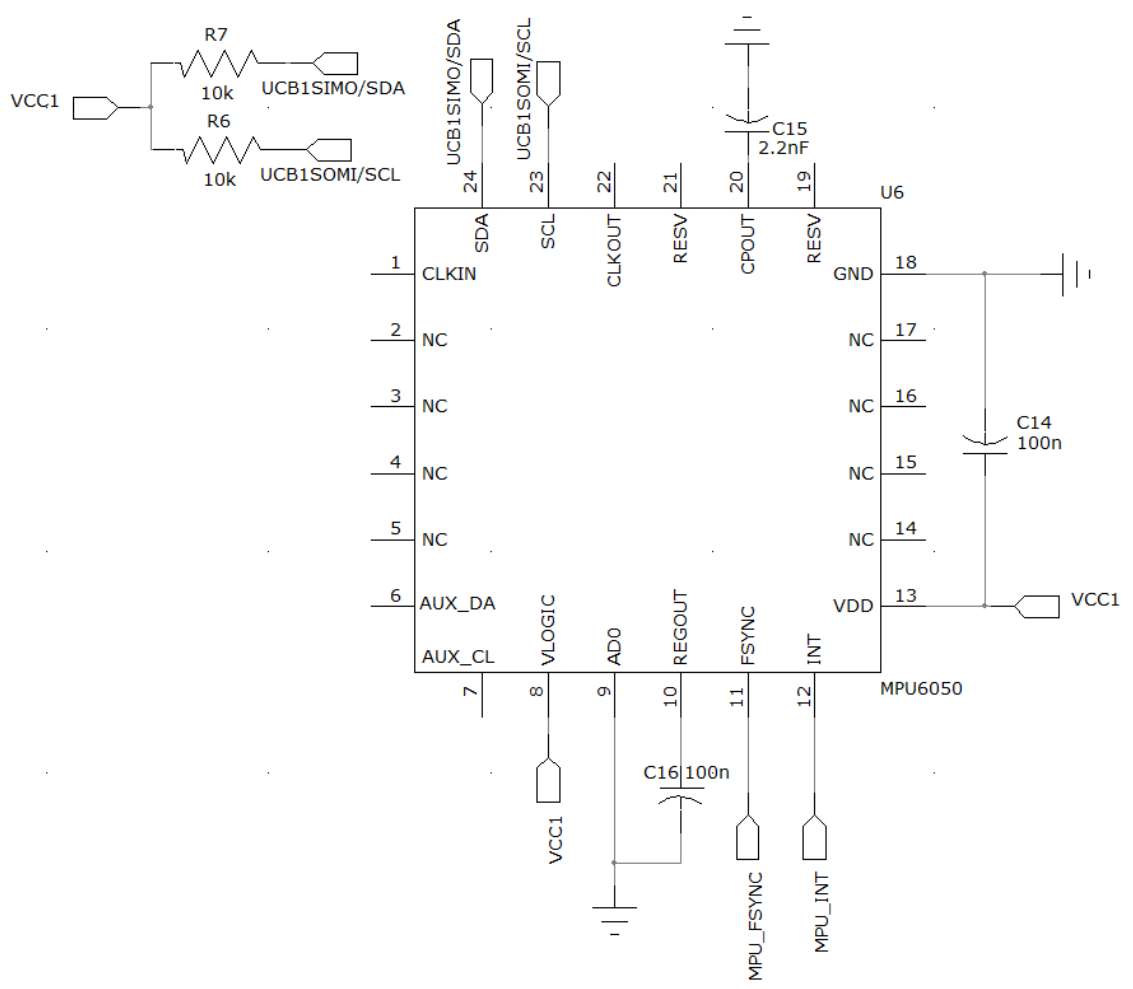

\section{MicroSD}

The on-board microSD connector along with affiliated SPI , card indicator, and supply connections is provided in the schematic below.

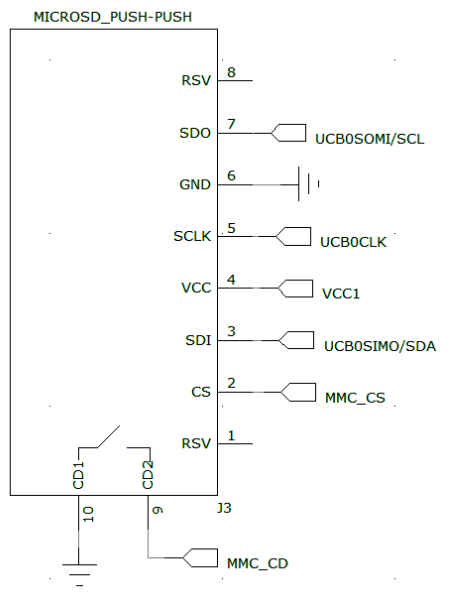




\section{Development Header}

The TEMPO 4 development header schematic view is shown below with appropriate main-board side connection made is provided in the schematic below.

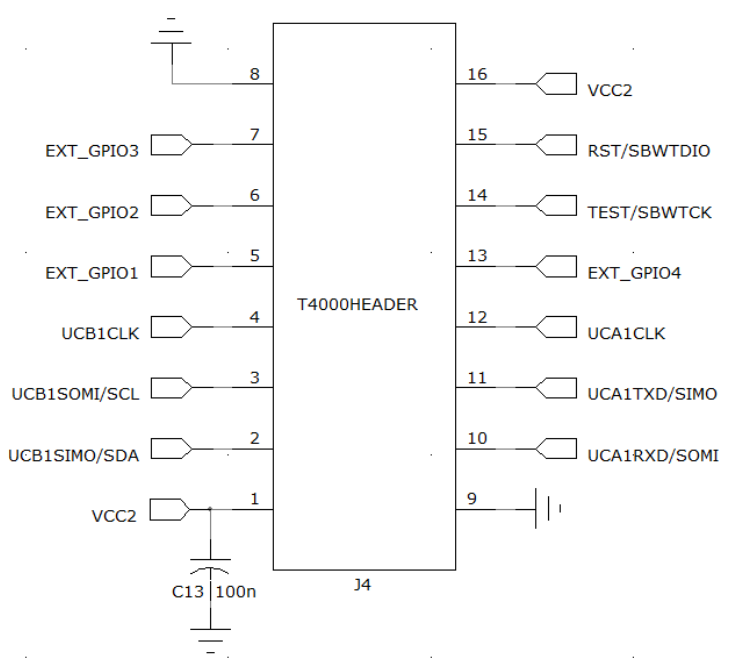




\section{System Controller and User I/O}

This schematic portion includes the MSP430 microcontroller along with the system programming interface, push-buttons, LEDs, crystal oscillator, and supply decoupling.

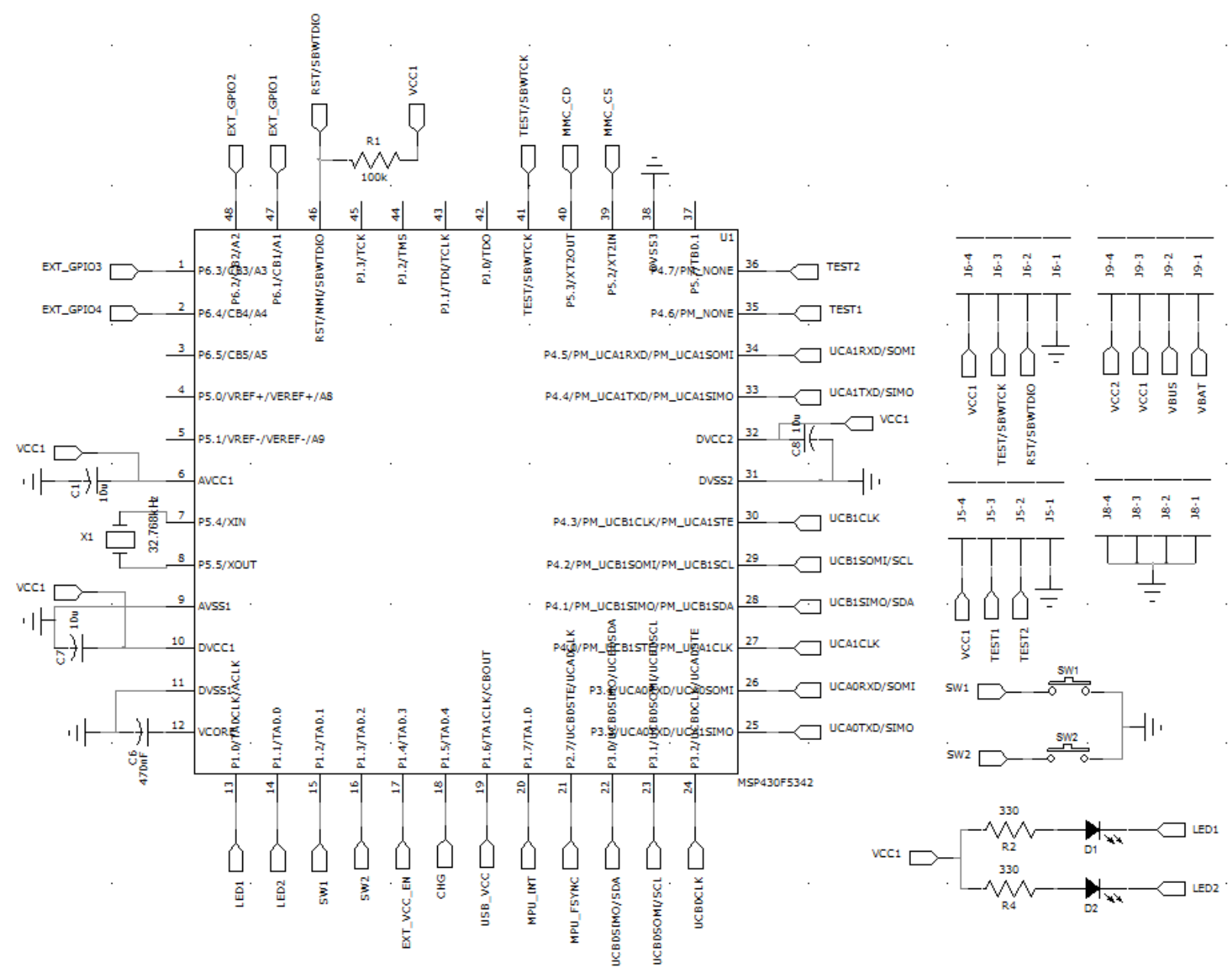




\section{Appendix B}

\section{Firmware Libraries}

This appendix presents the core firmware libraries developed as part of this work. In addition a number of other, more specialized code-routines were also developed for the purpose of verification and measurement. Not all of this code is included in this section.

\section{Clocks.h}

This file contains clock rate definitions and macros for blocking delay routines

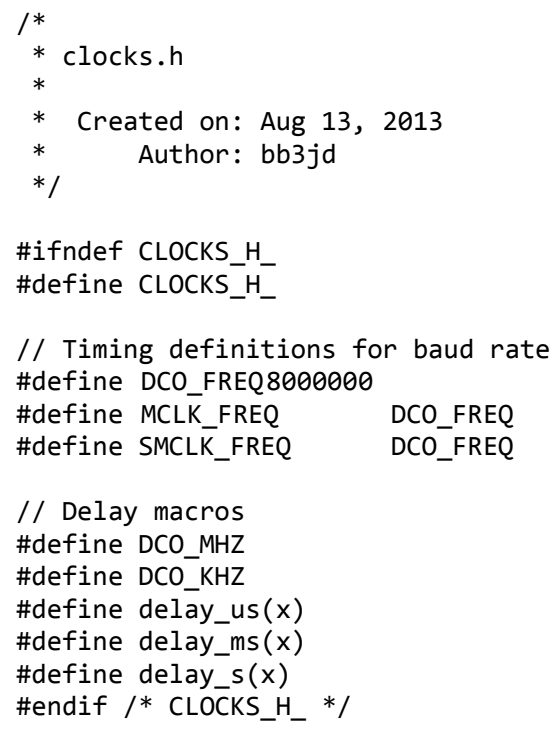

$/ / /<$ DCO frequency (you should call setFLL(DCO_FREQ))

$/ / /<$ Main clock frequency (change if using MCLK_DIV > 1 )

$/ / /<$ Sub-main clock frequency (change if using SMCLK_DIV >1)

DCO FREQ/1000000 $\quad / / /<$ DCO Rate in $\mathrm{MHz}$

DCO FREQ/1000 $/ / /<$ DCO rate in $\mathrm{kHz}$

_delay_cycles ( $x *$ DCO_MHZ) $/ / /<$ Blocking delay macro (in us)

_delay_cycles( $x *$ DCO_KHZ) $/ / /<$ Blocking delay macro (in ms)

_delay_cycles $(x *$ DCO_FREQ) $/ / /<$ Blocking delay macro (in $s$ )

\section{Comm.c}

This file contains core communications library management code and interrupt routines for USCI peripheral modules in the MSP430

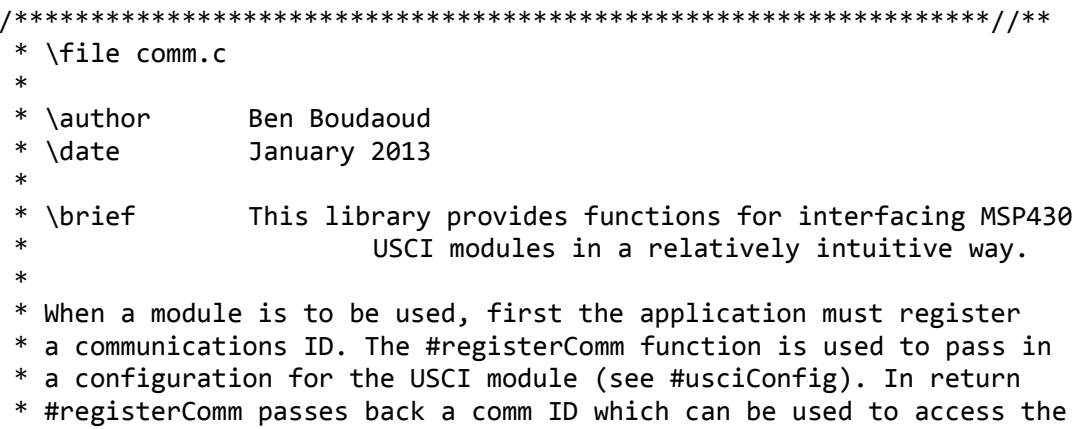


* USCI. Currently the library supports UART, SPI, and I2C single master

$*$ modes.

$* * * * * * * * * * * * * * * * * * * * * * * * * * * * * * * * * * * * * * * * * * * * * * * * * * * * * * * * * * * * * * * * * * * * * /$

\#include "comm.h"

usciConfig *dev[MAX_DEVS]; $\quad / / /<$ Device config buffer (indexed by comm ID always non-zero)

unsigned int devIndex $=0$;

unsigned int devConf $[4]=\{0,0,0,0\} ; \quad / / /<$ Currently applied configs buffer $[A \theta, A 1, B 0, B 1]$

usciStatus uscistat $[4]=\{$ OPEN, OPEN, OPEN, OPEN $\} ; / / /<$ Store status (OPEN, TX, or RX) for [A0, A1, B0, B1]

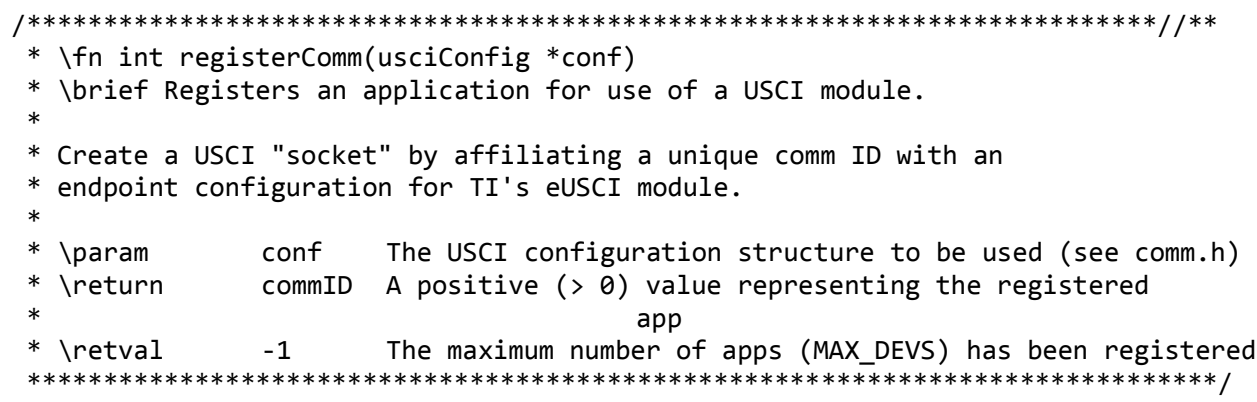

int registerComm(usciConfig * conf)

\{
if(devIndex >= MAX_DEVS) return -1 ;
// Check device list not full
$\operatorname{dev}[++\operatorname{devIndex}]=$ conf;
// Copy config pointer into device list

\} return devIndex;

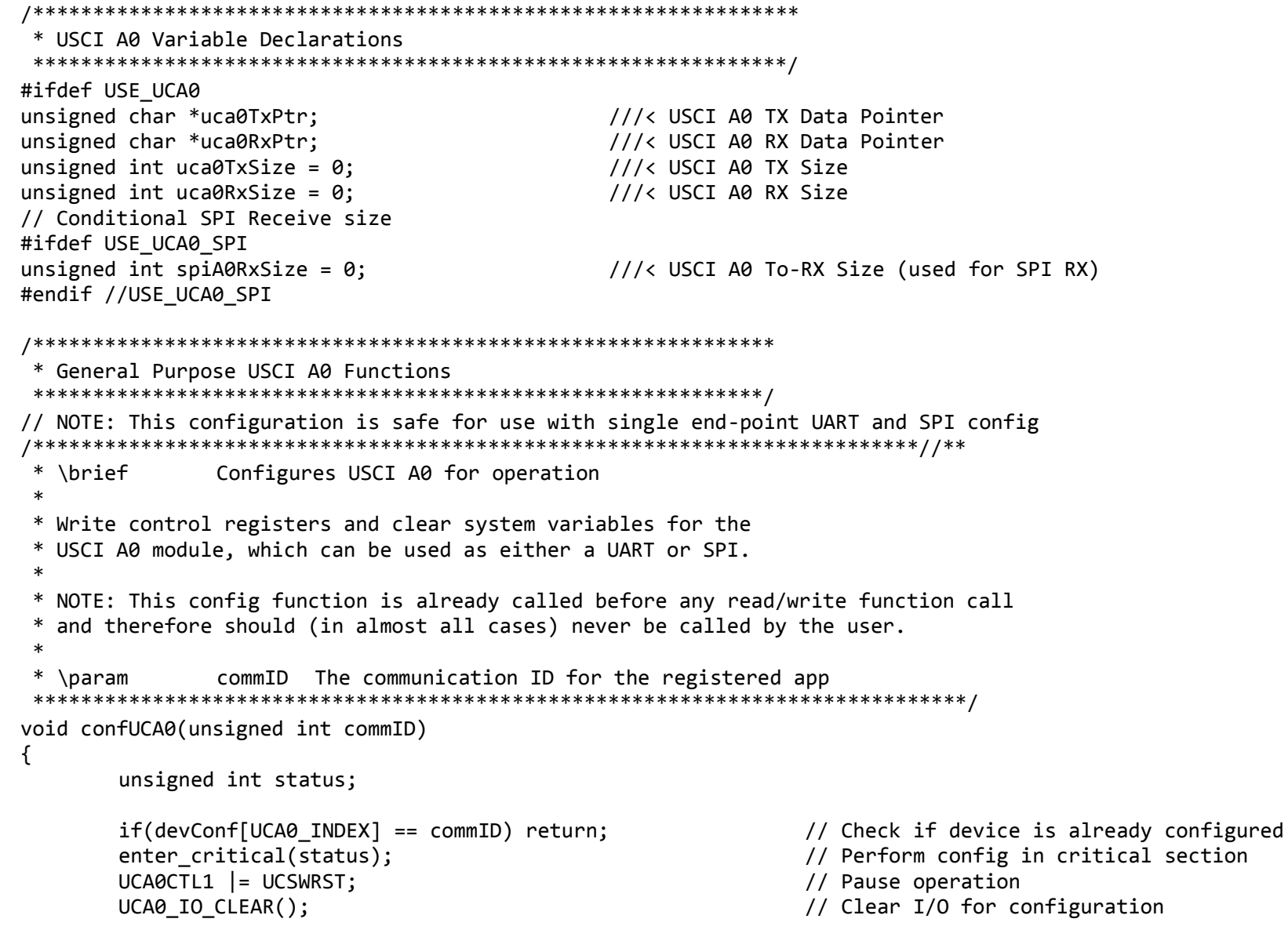

// Check if device is already configured

// Perform config in critical section

// Pause operation

// Clear I/O for configuration 


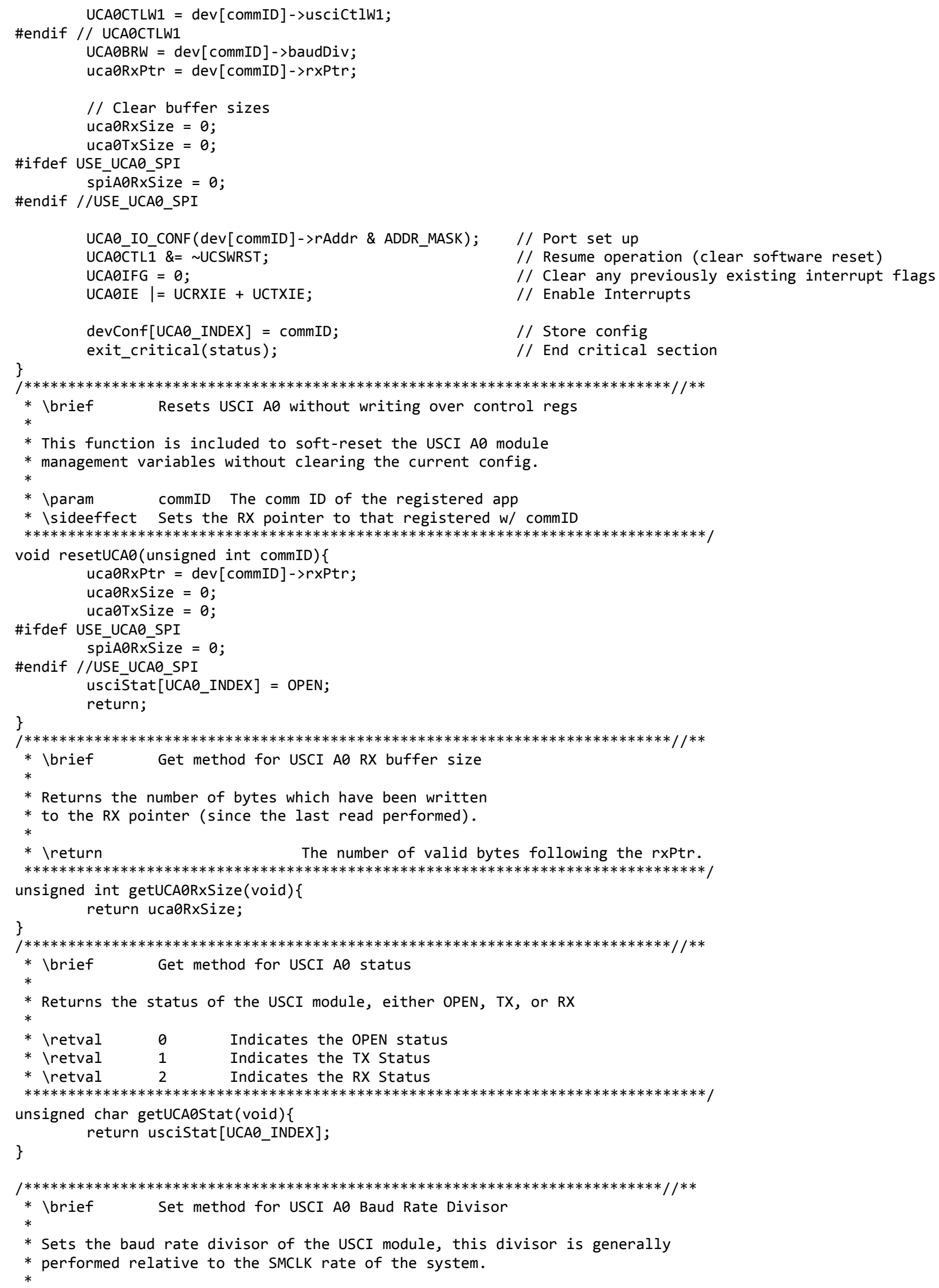




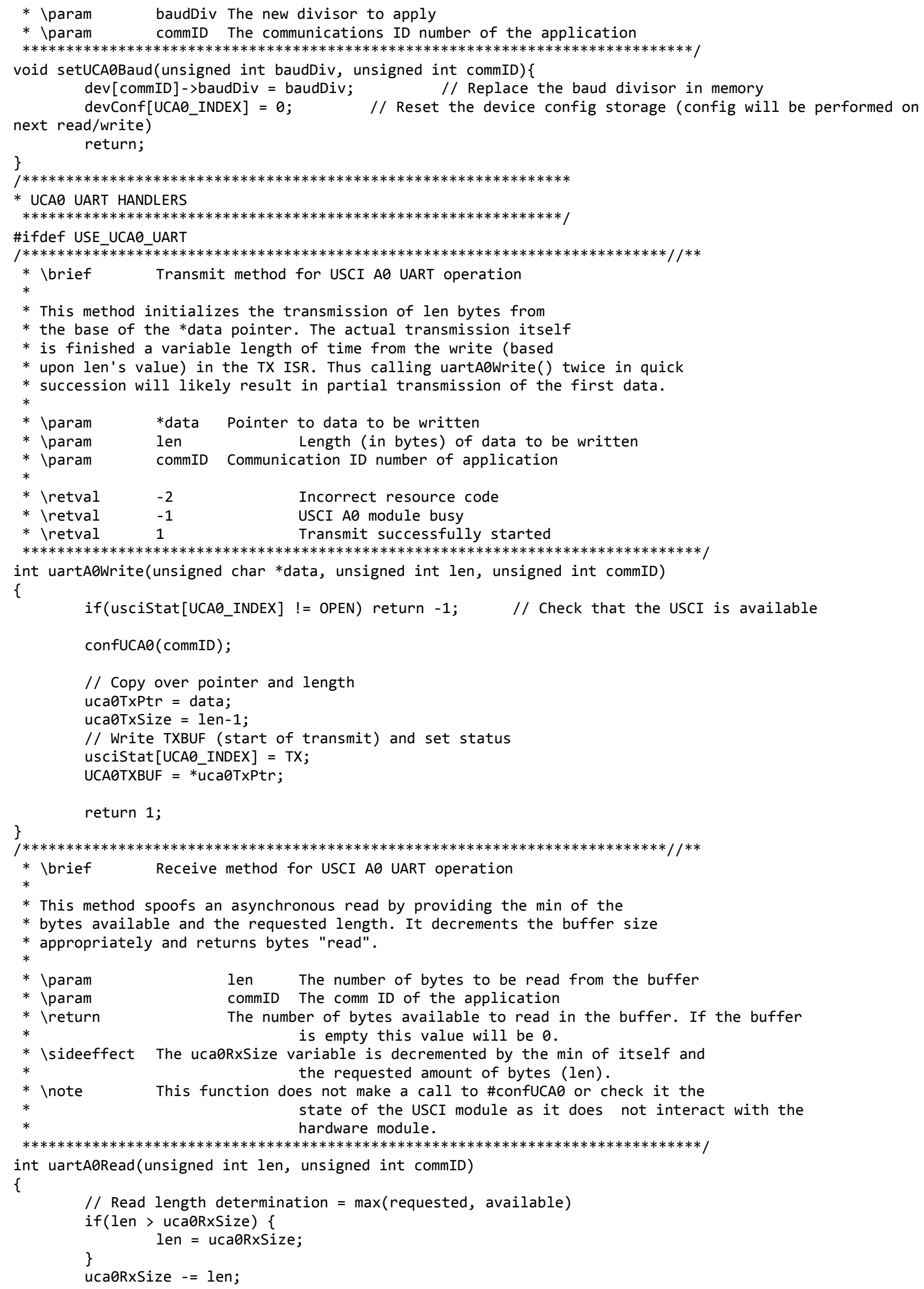




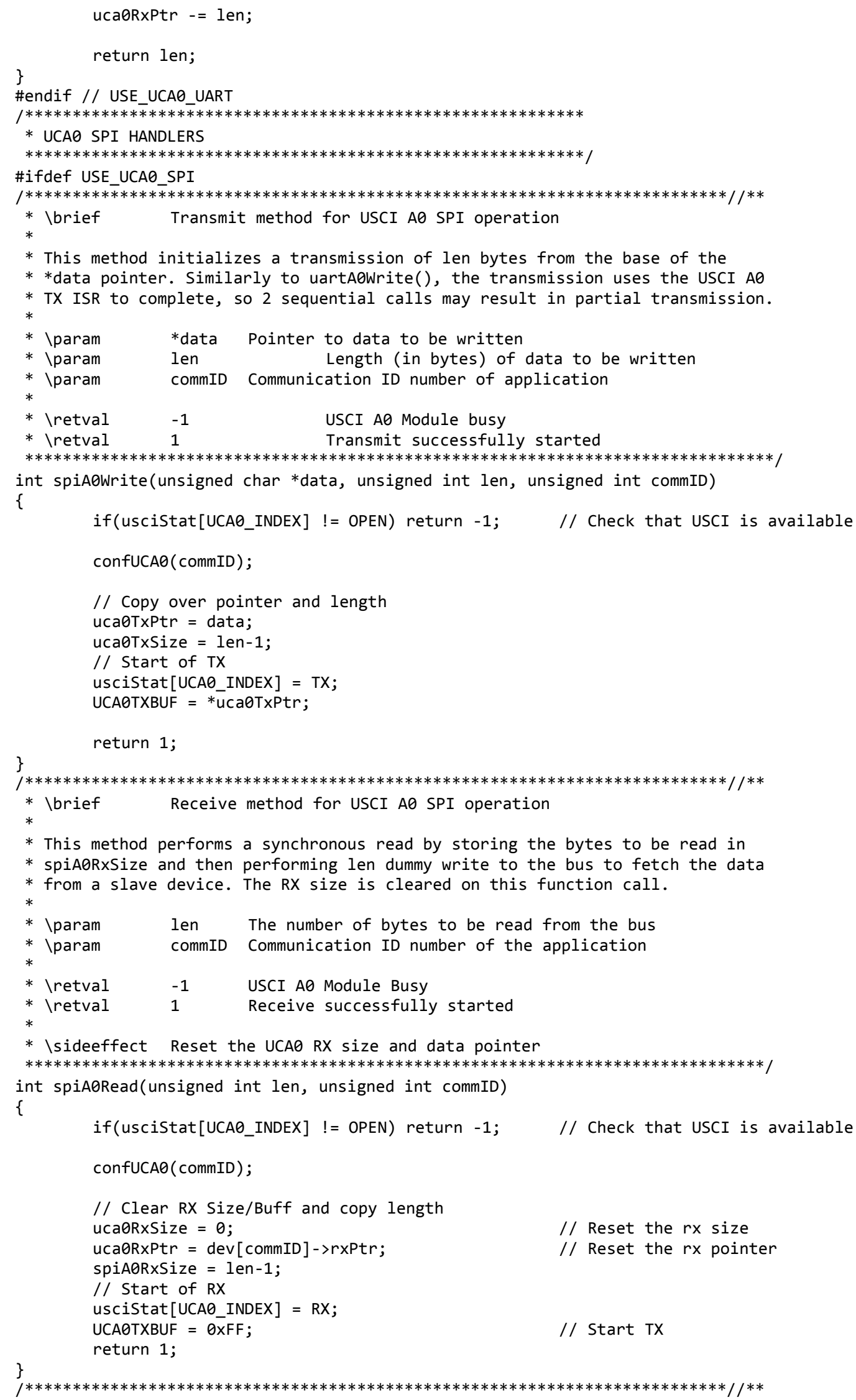




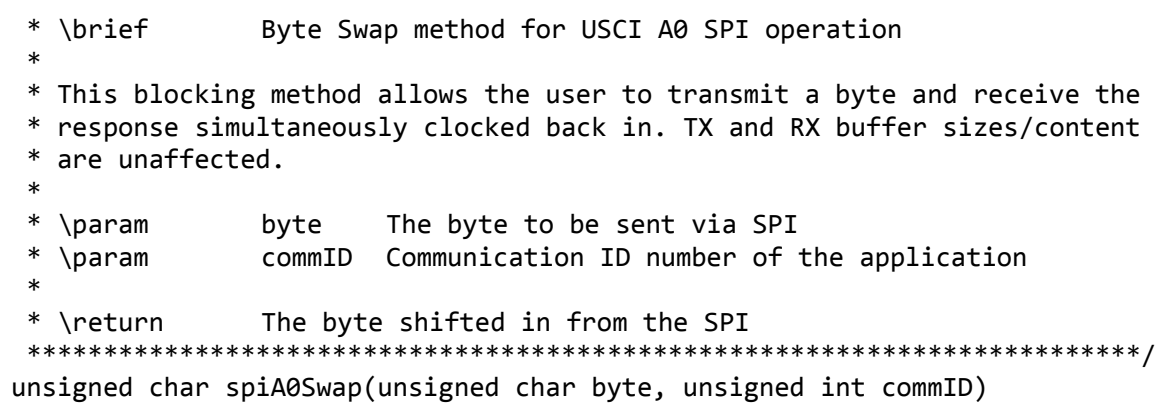




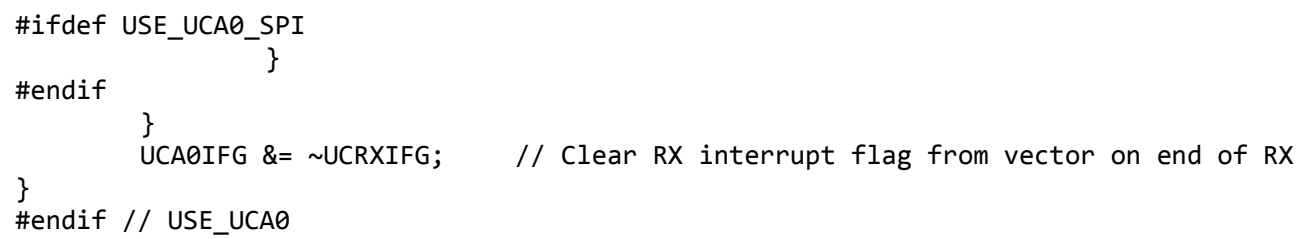




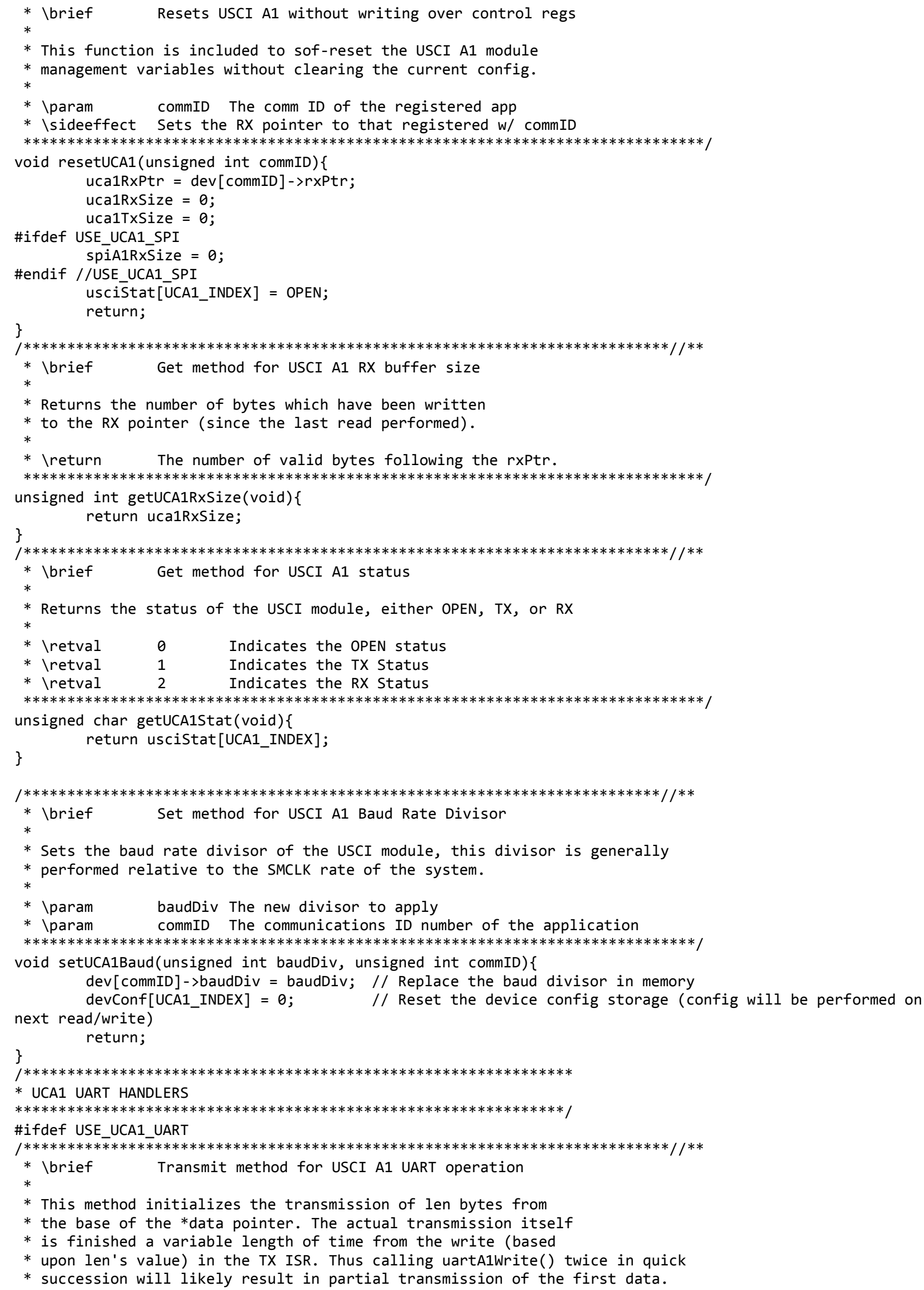




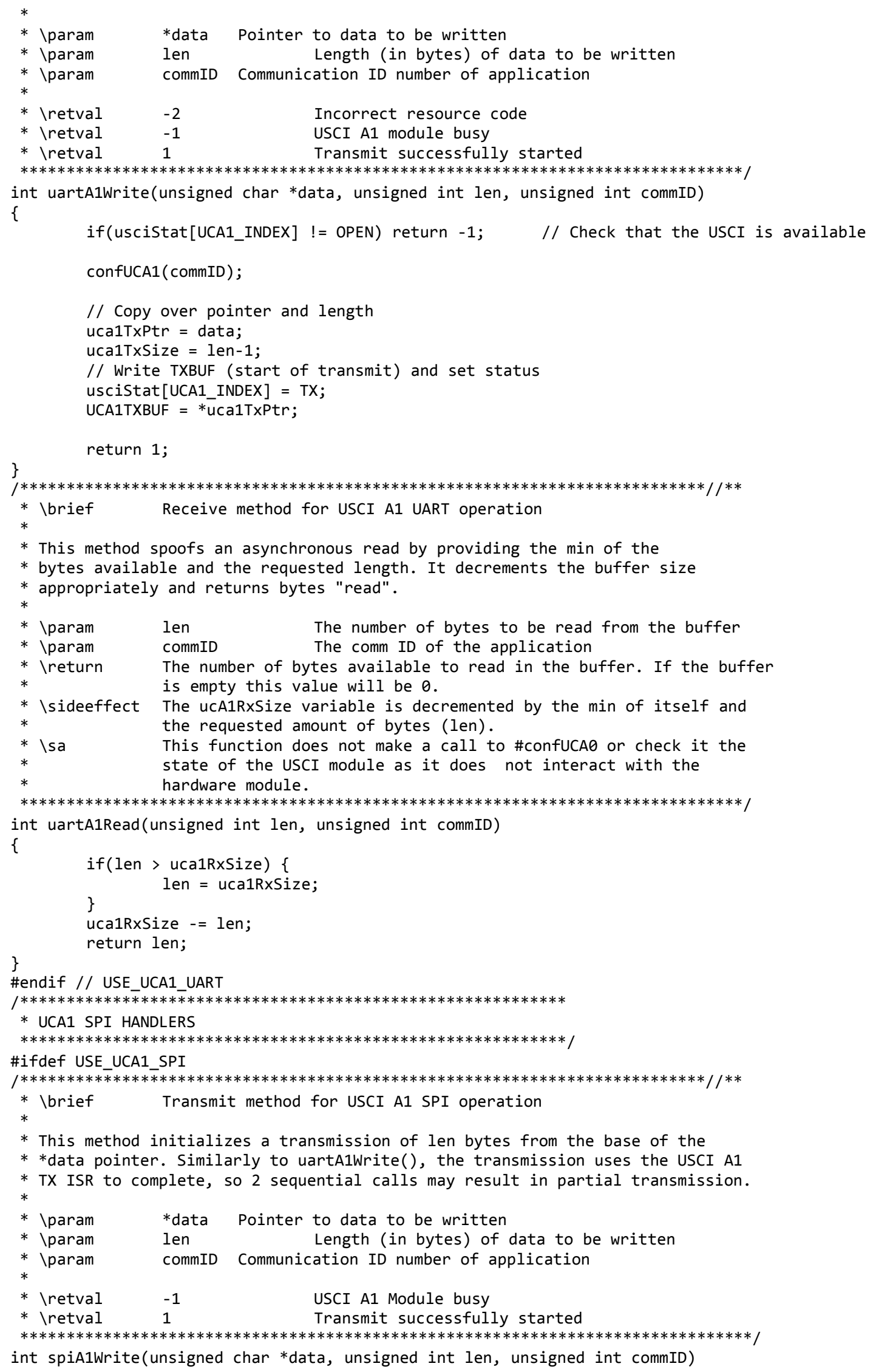




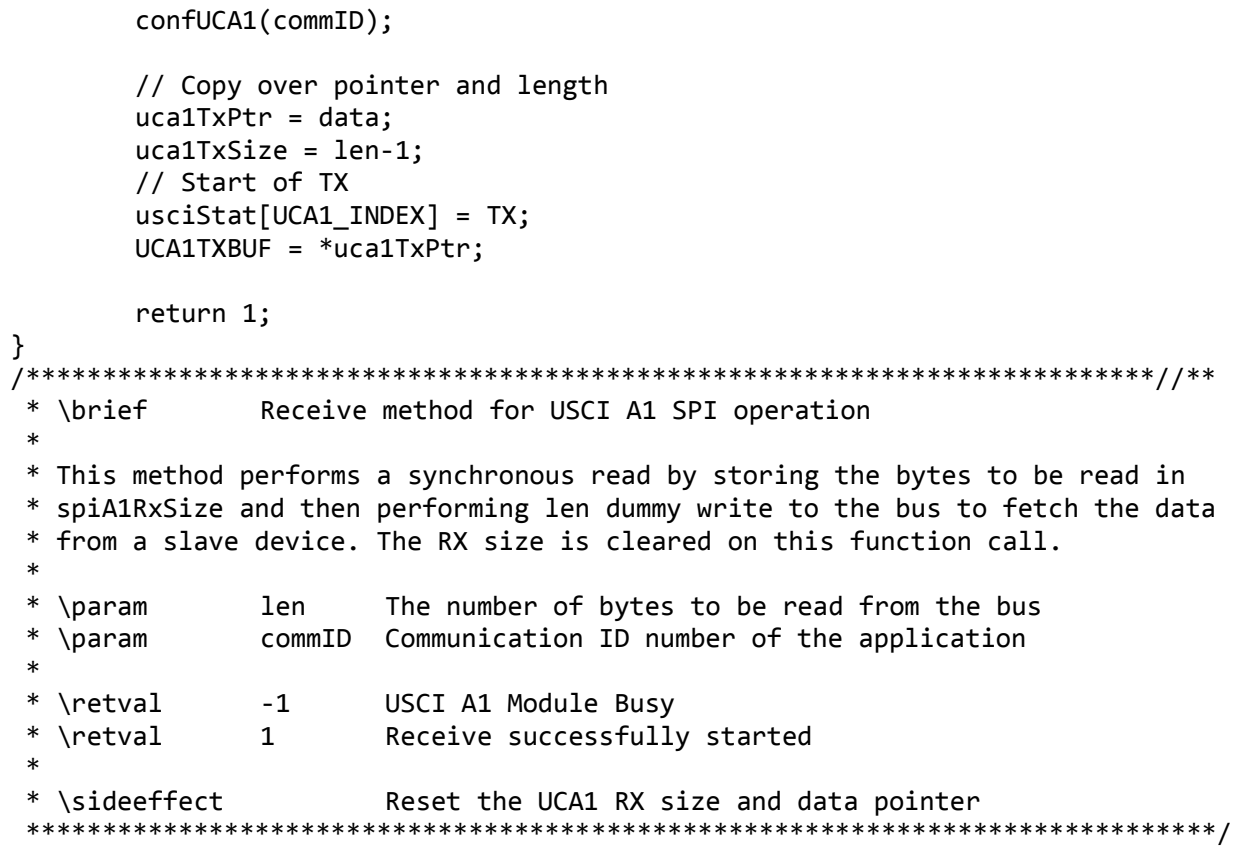


\#endif //USE_UCA1_SPI

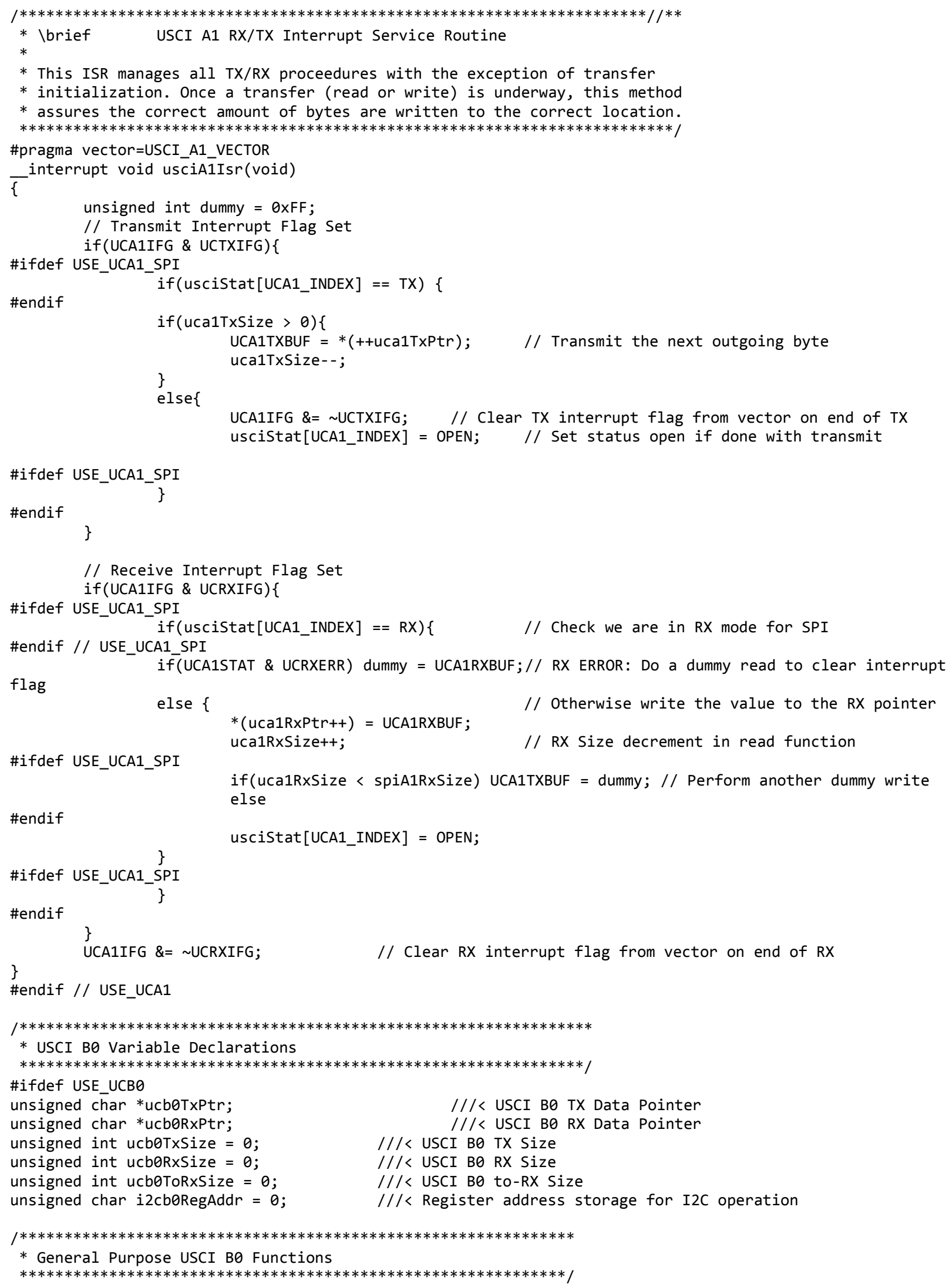




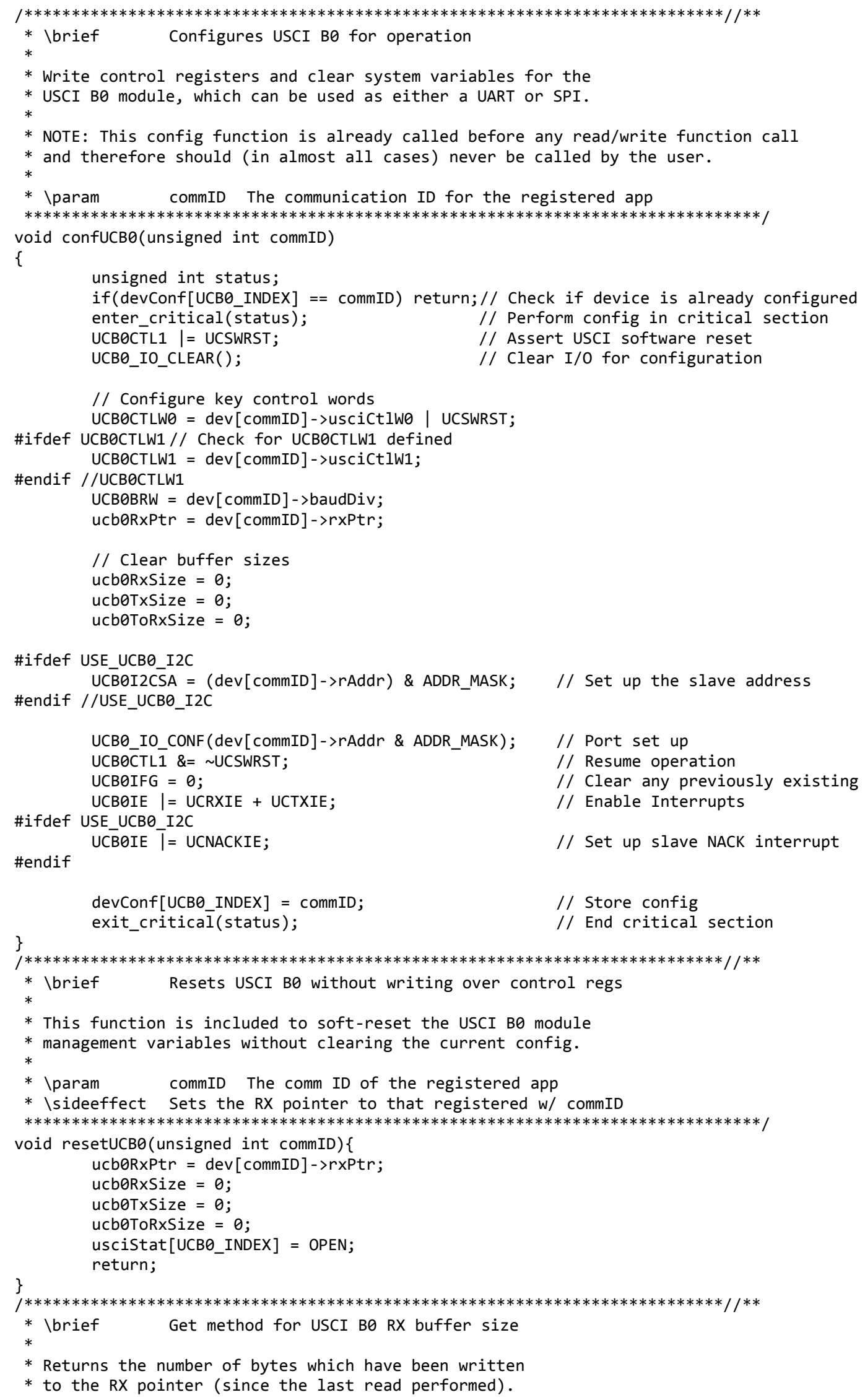




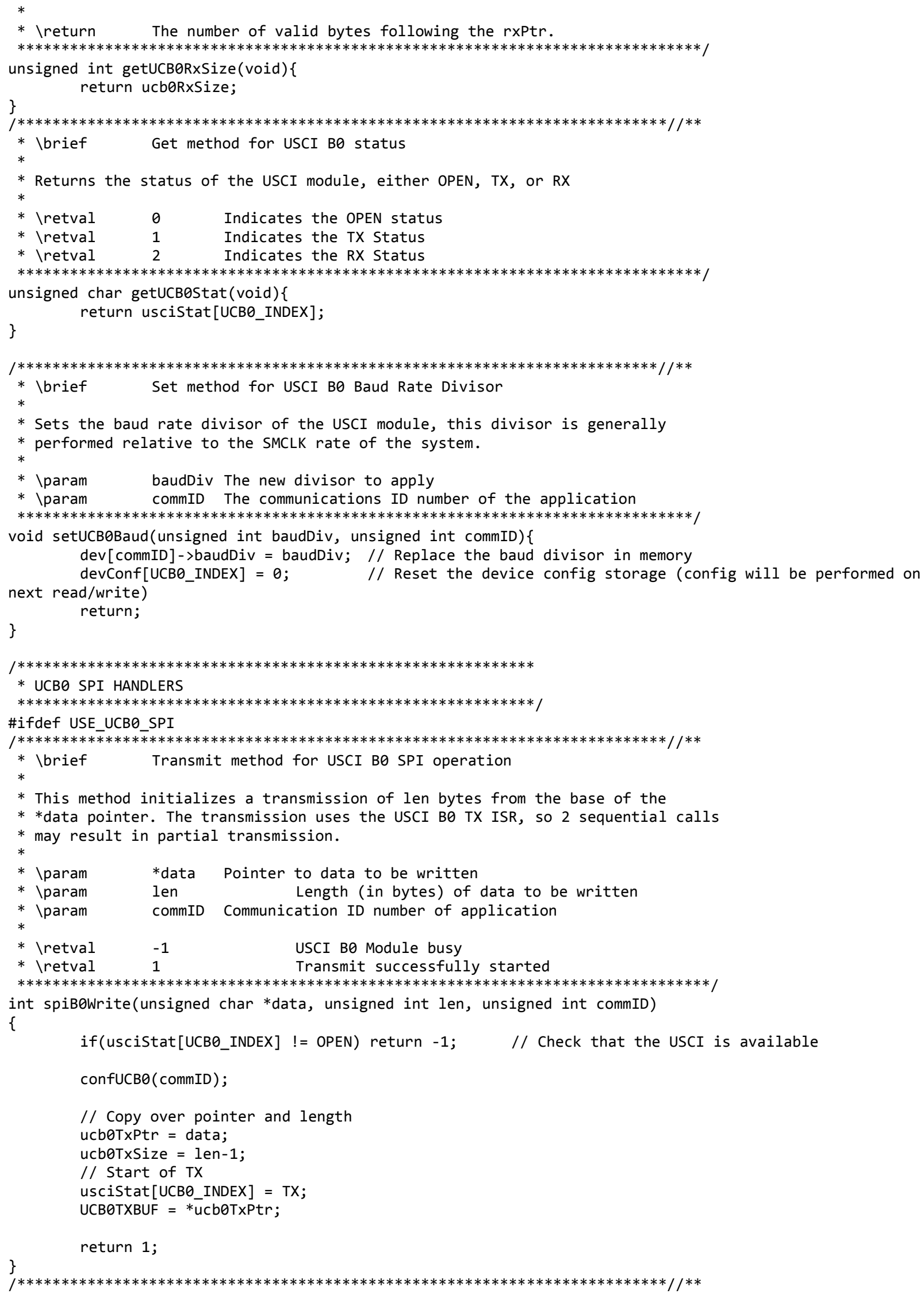




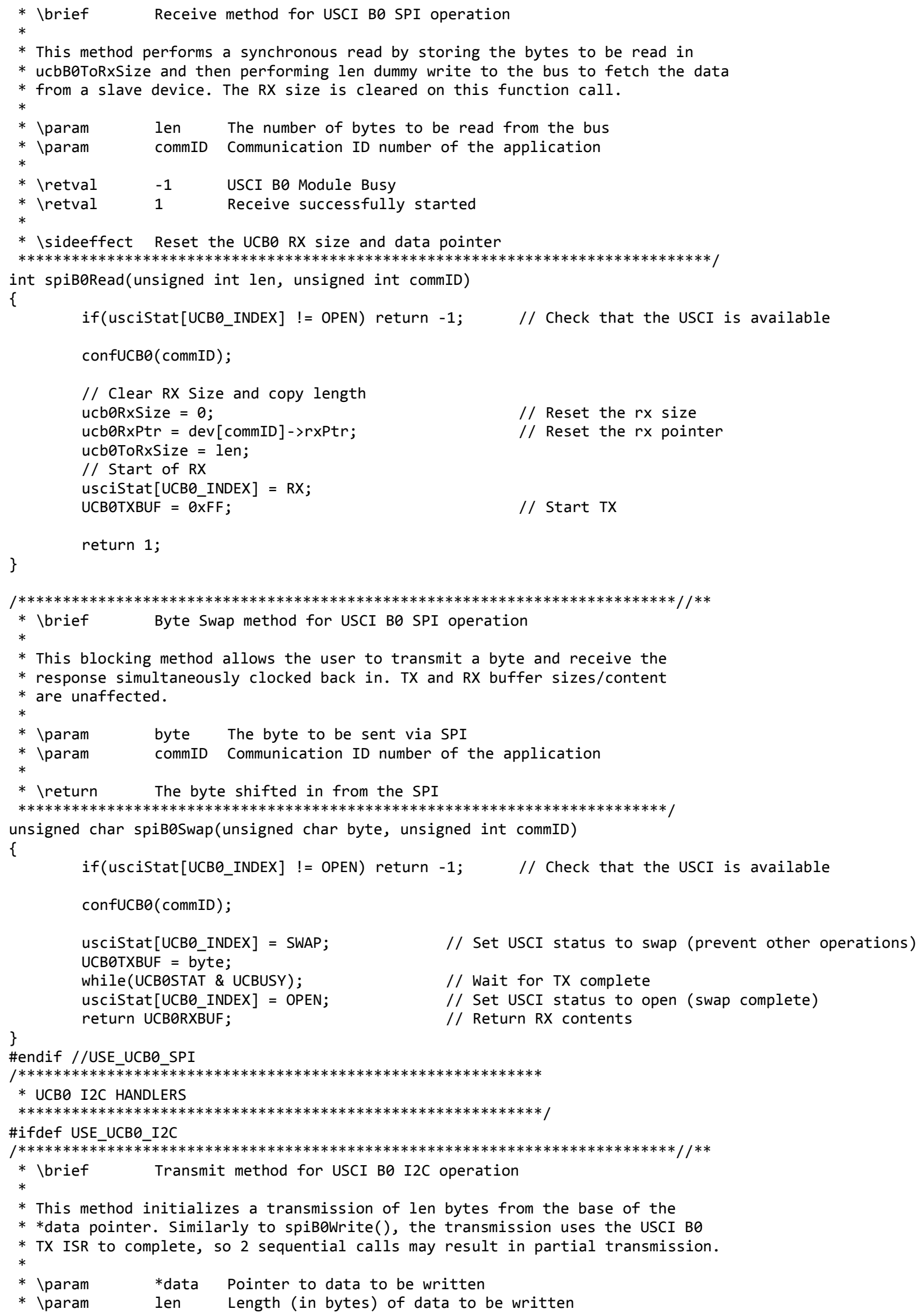




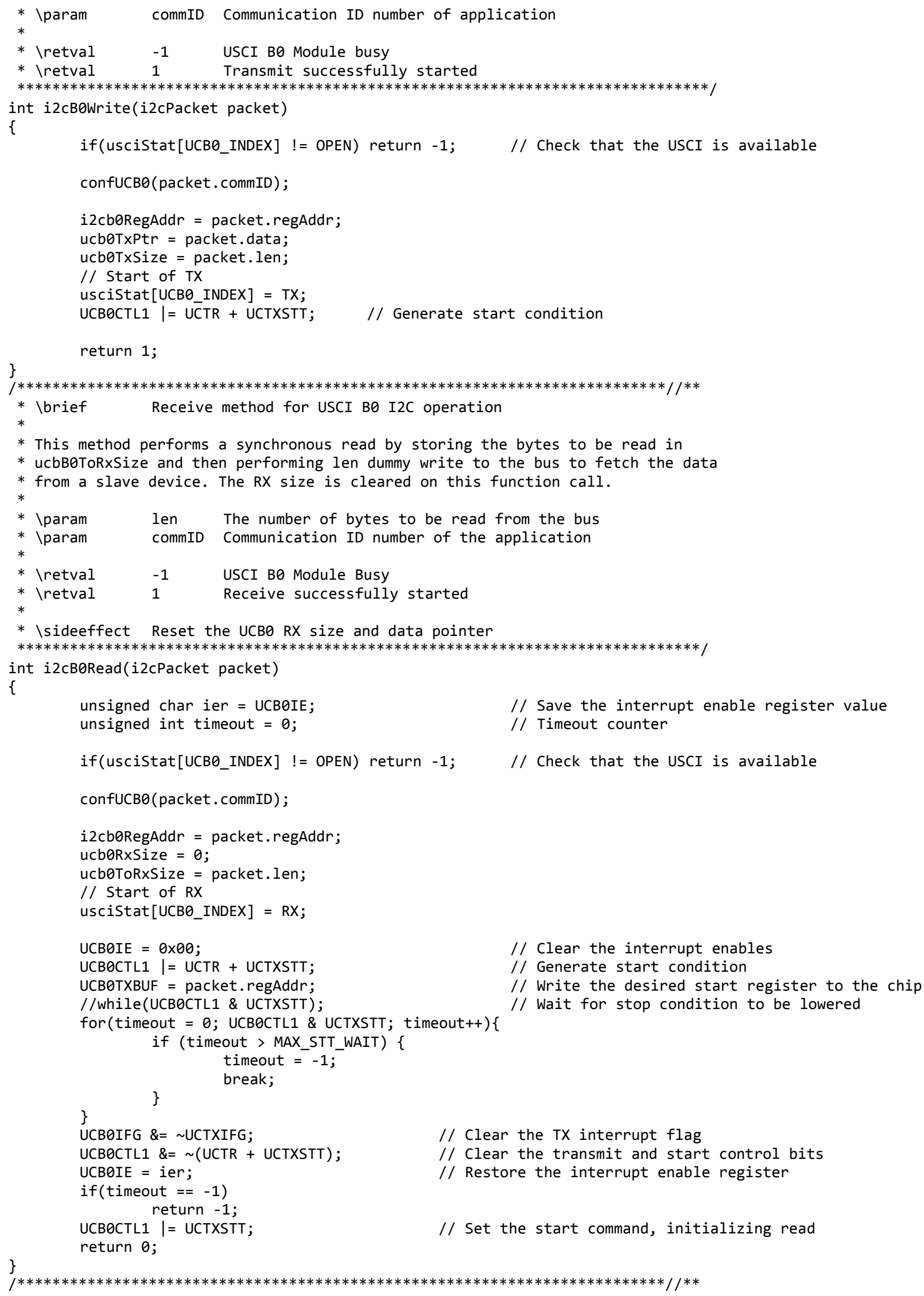

// Clear the interrupt enables

// Generate start condition

// Write the desired start register to the chip

// Wait for stop condition to be lowered \} 


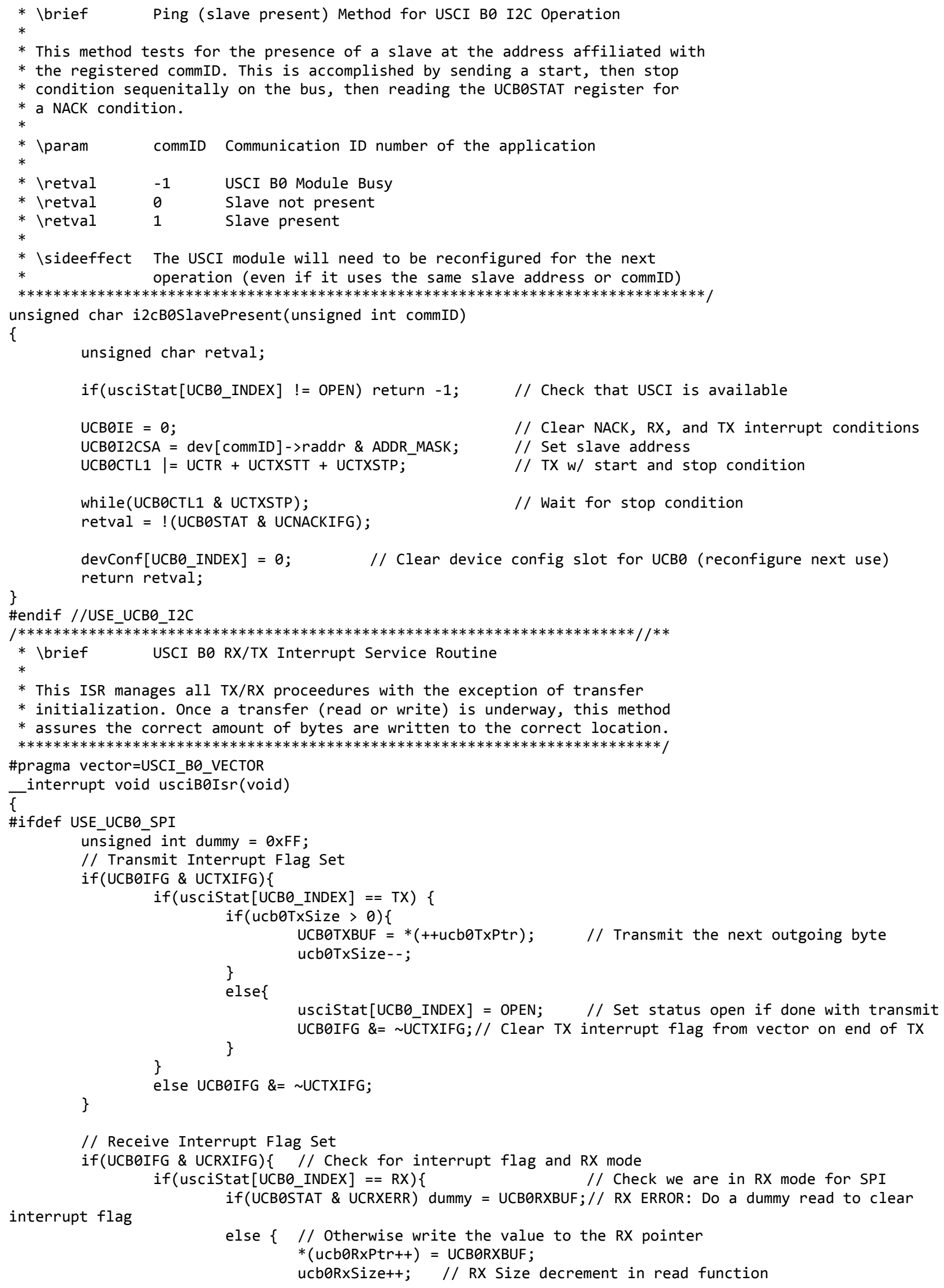


write

if(ucb0RxSize < ucb0ToRxSize) UCBOTXBUF = dummy; // Perform another dummy

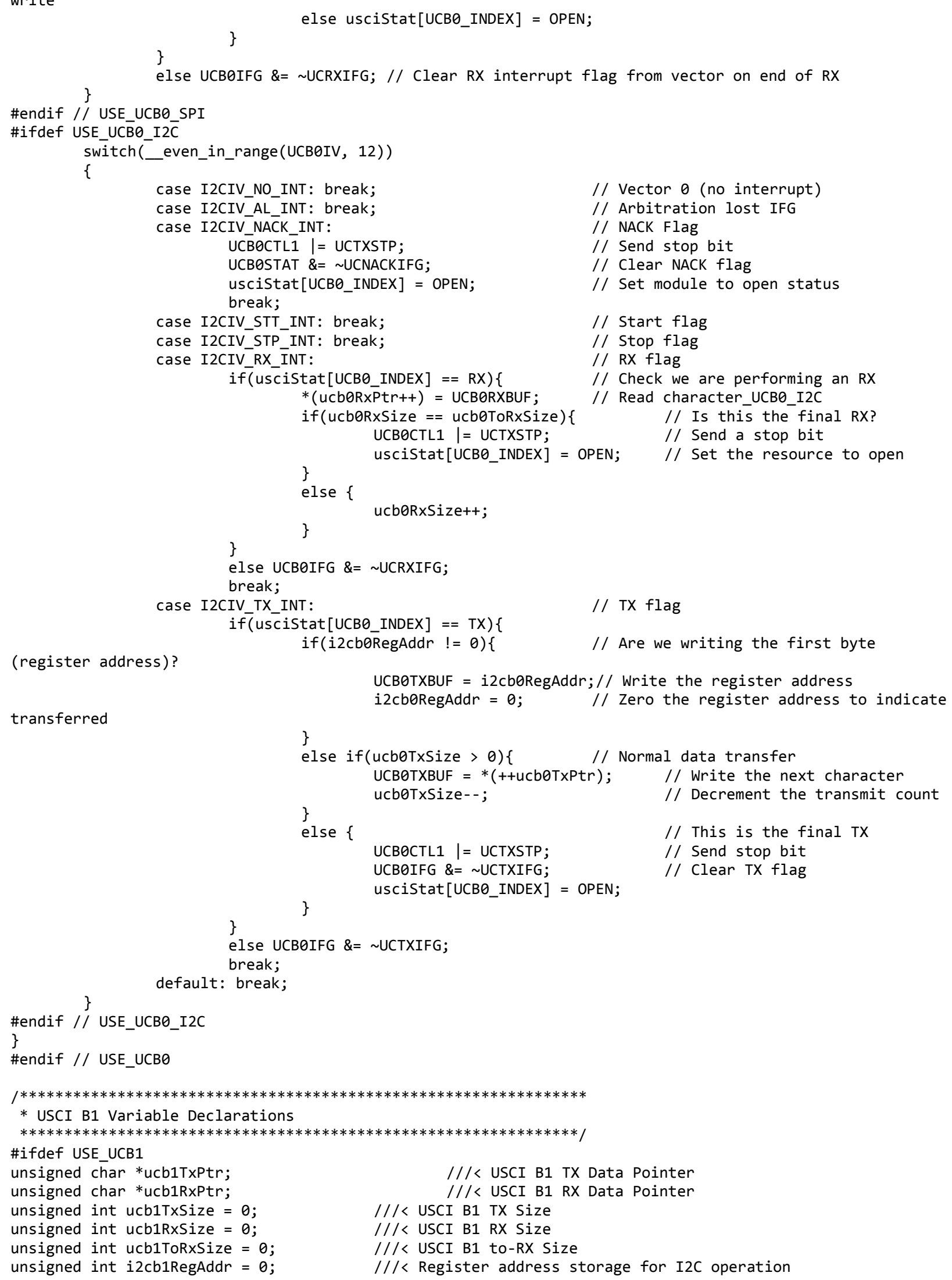

$/ / /<$ USCI B1 TX Data Pointer

$/ / /<$ USCI B1 RX Data Pointer

$/ / /<$ USCI B1 TX Size

$/ / /<$ USCI B1 RX Size

$/ / /<$ USCI B1 to-RX Size

$/ / /<$ Register address storage for I2C operation 


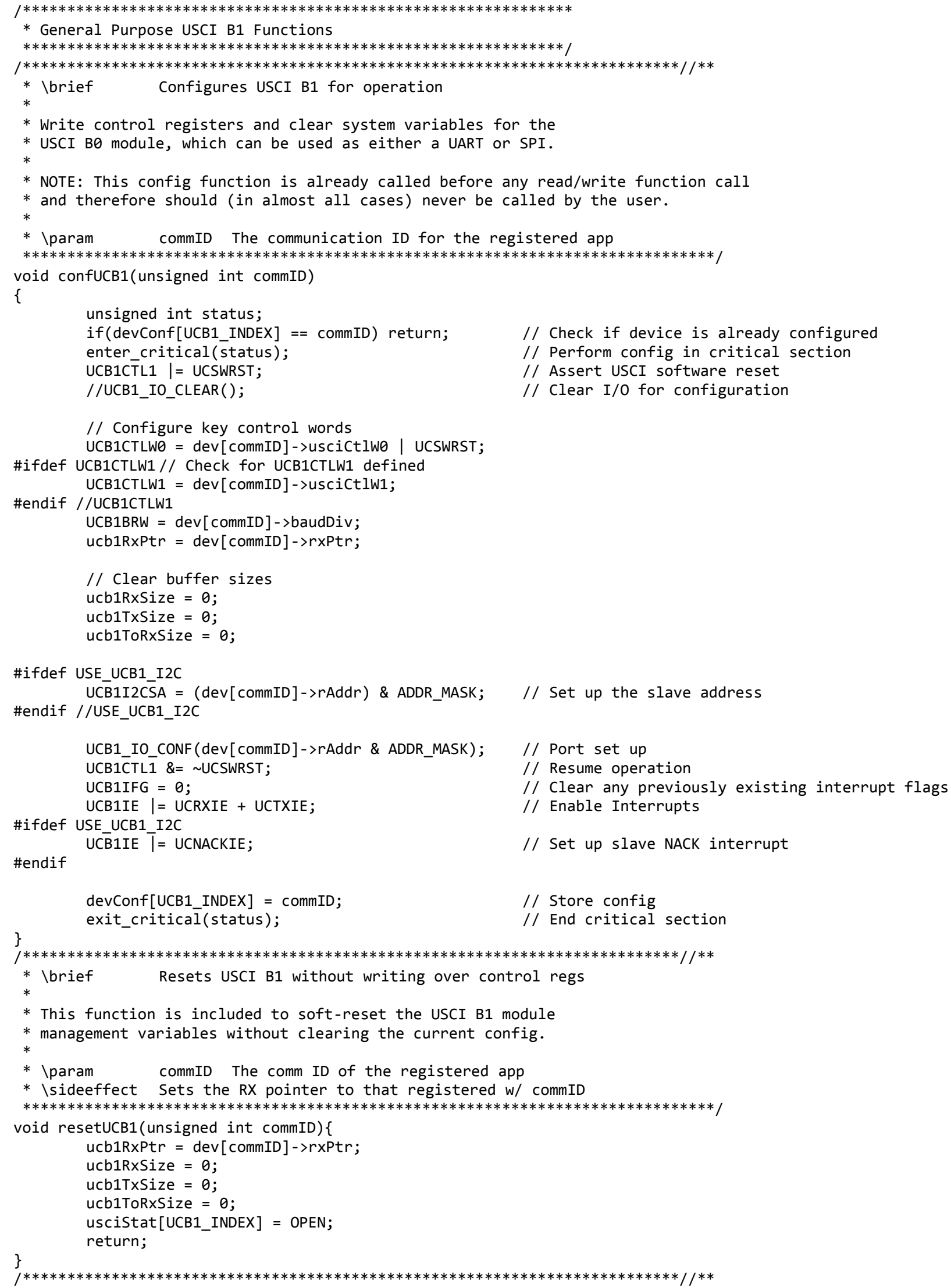




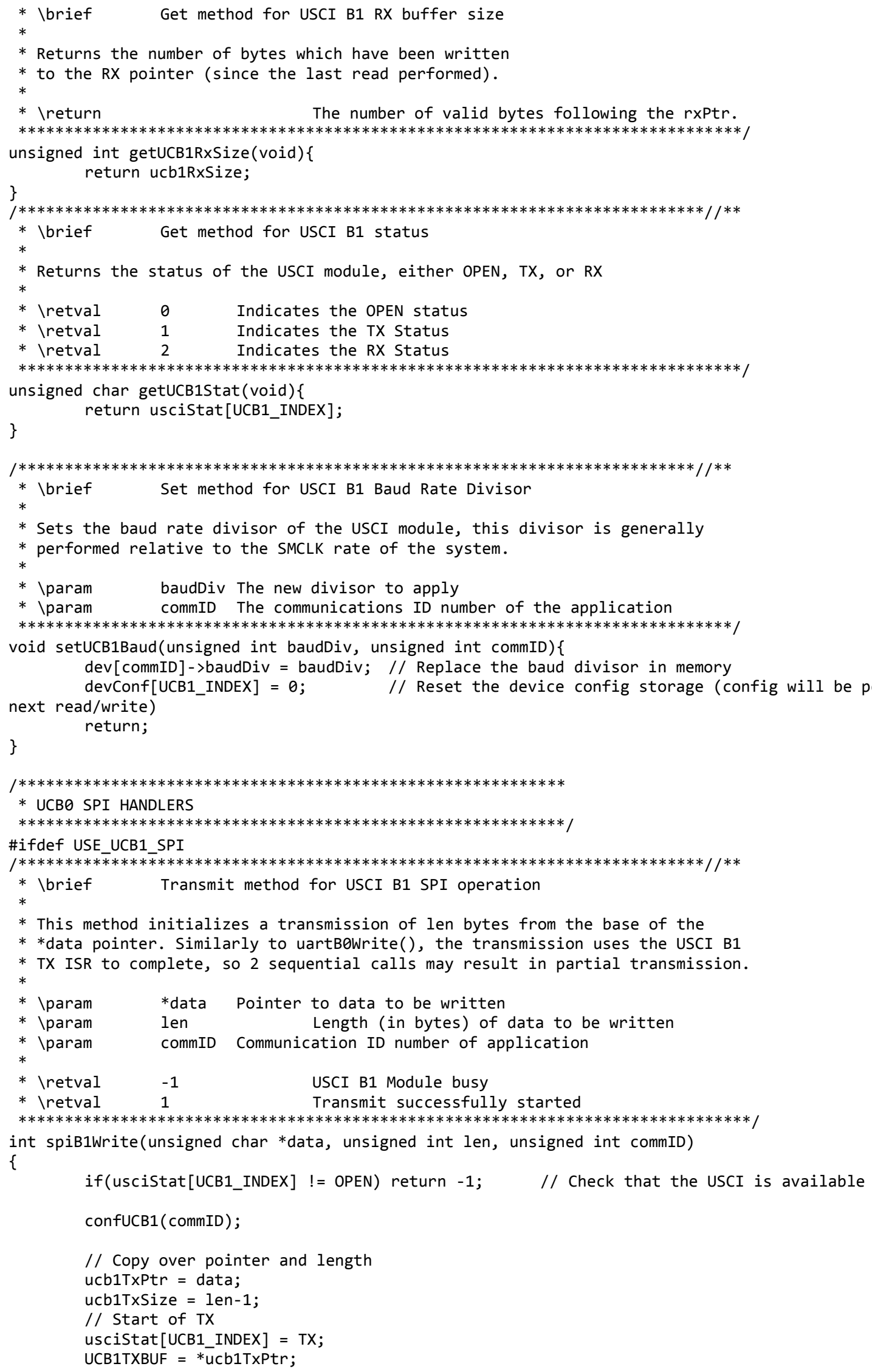


\}

return 1;

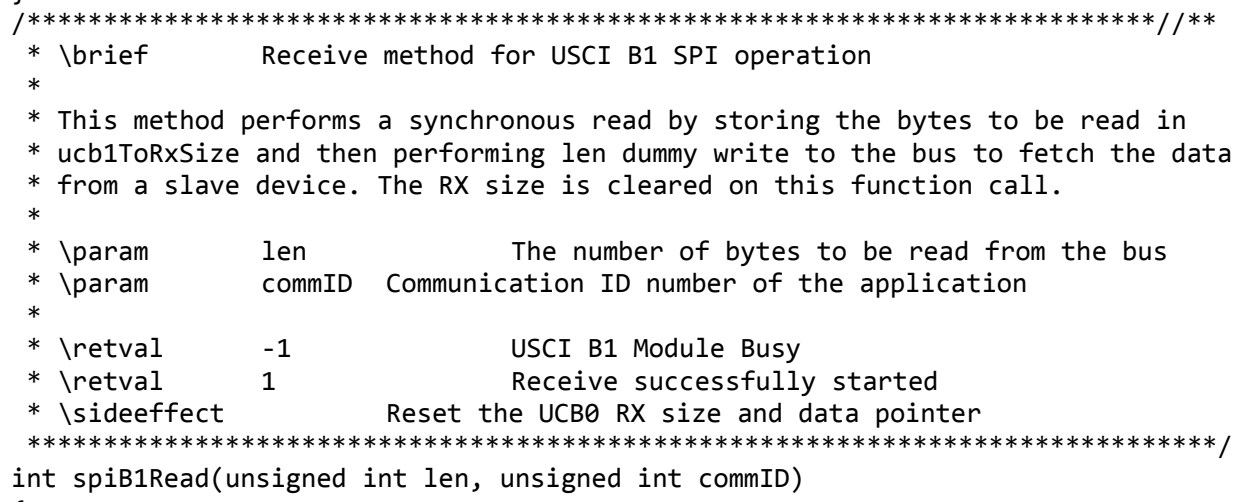

int spiB1Read(unsigned int len, unsigned int commID)

\{

if(uscistat[UCB1_INDEX] != OPEN) return -1; // Check that the USCI is available

ConfUCB1( CommID);

// Clear RX Size and copy length

ucb1RxPtr $=\operatorname{dev}[$ commID]->rxPtr; $\quad$ // Reset the $r x$ pointer

ucb1RxSize $=0$;

ucb1ToRxSize = len-1;

// Start of RX

uscistat[UCB1_INDEX] = RX;

UCB1TXBUF = OXFF; $\quad$ // start TX

\}

return 1;

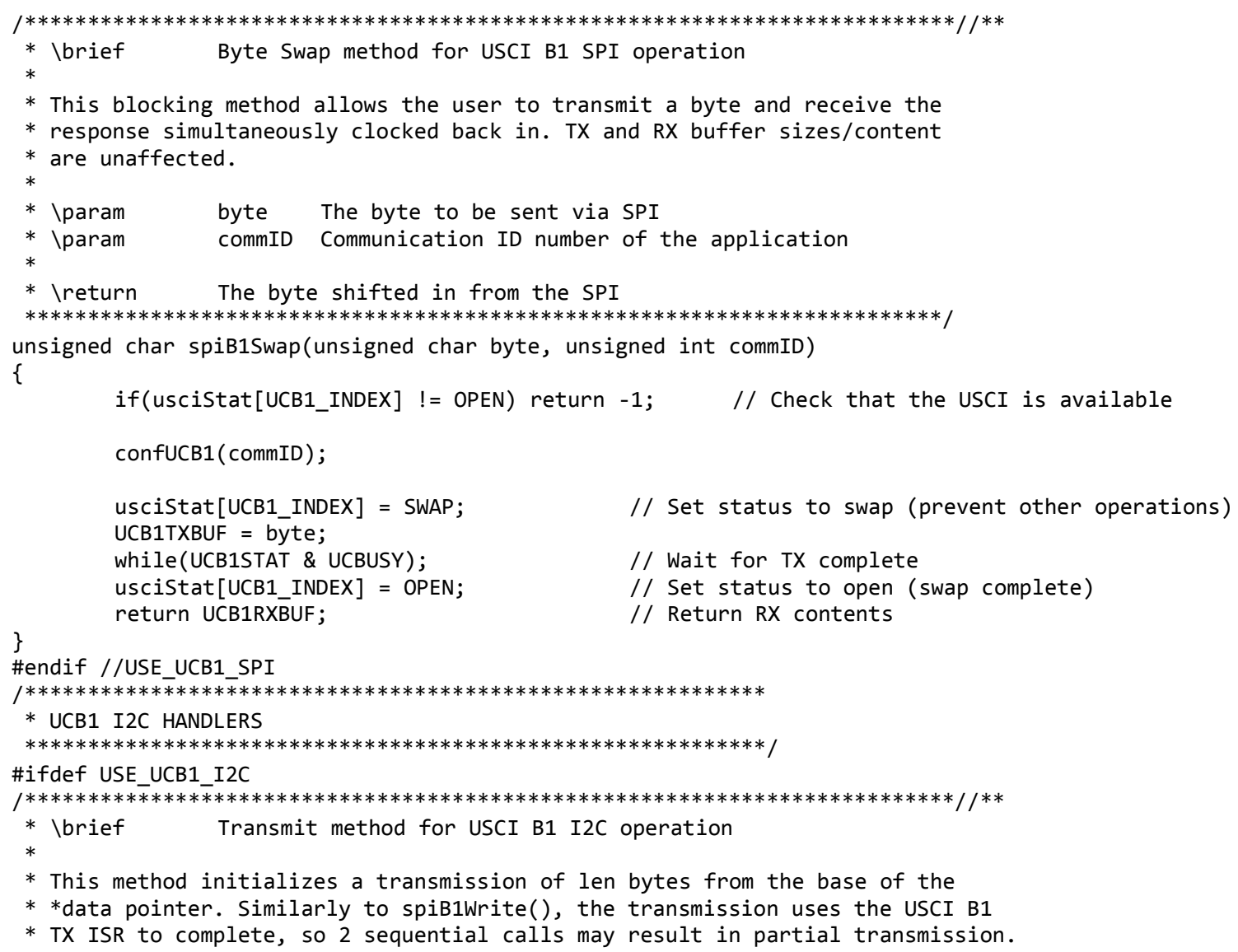




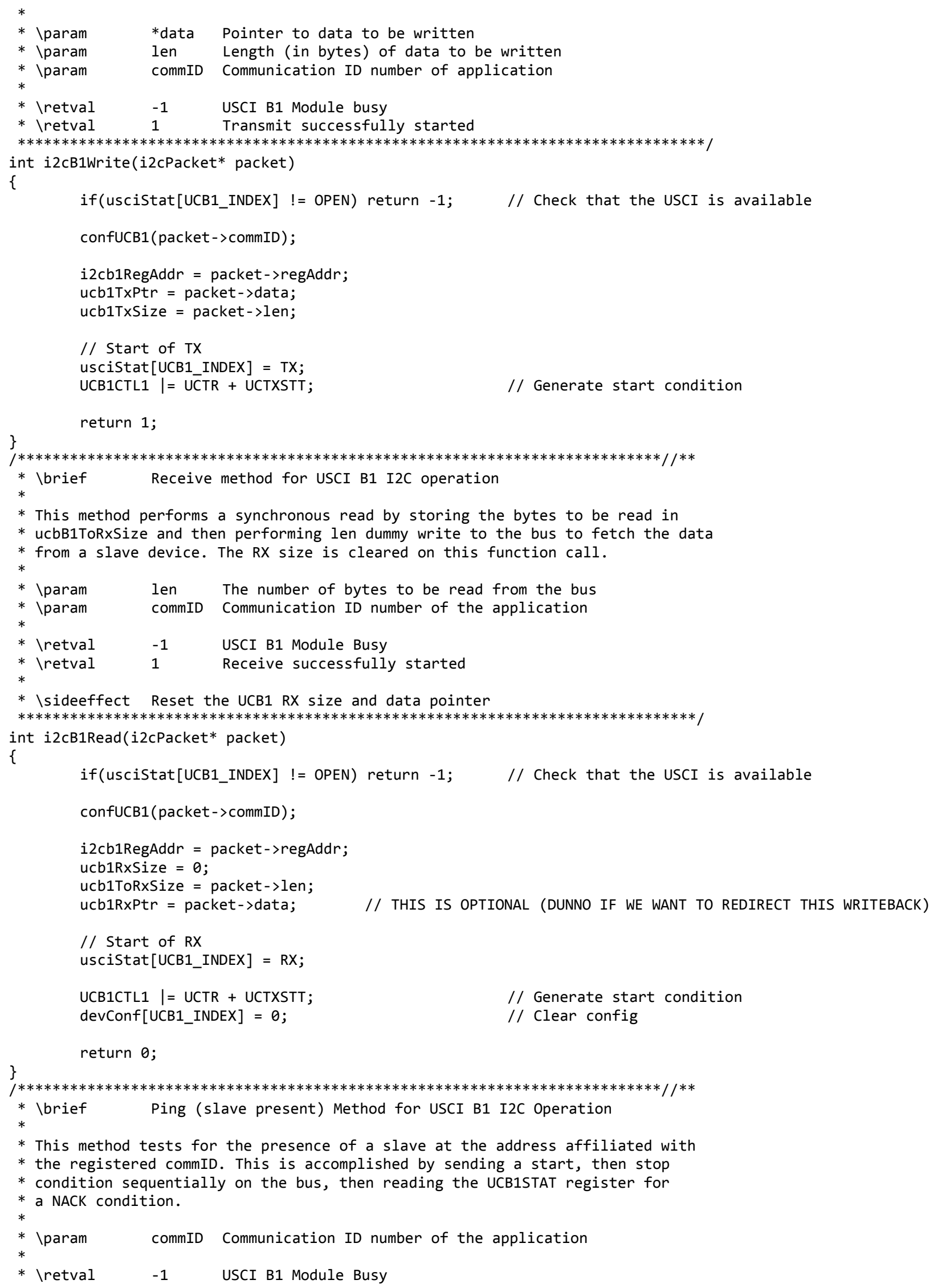




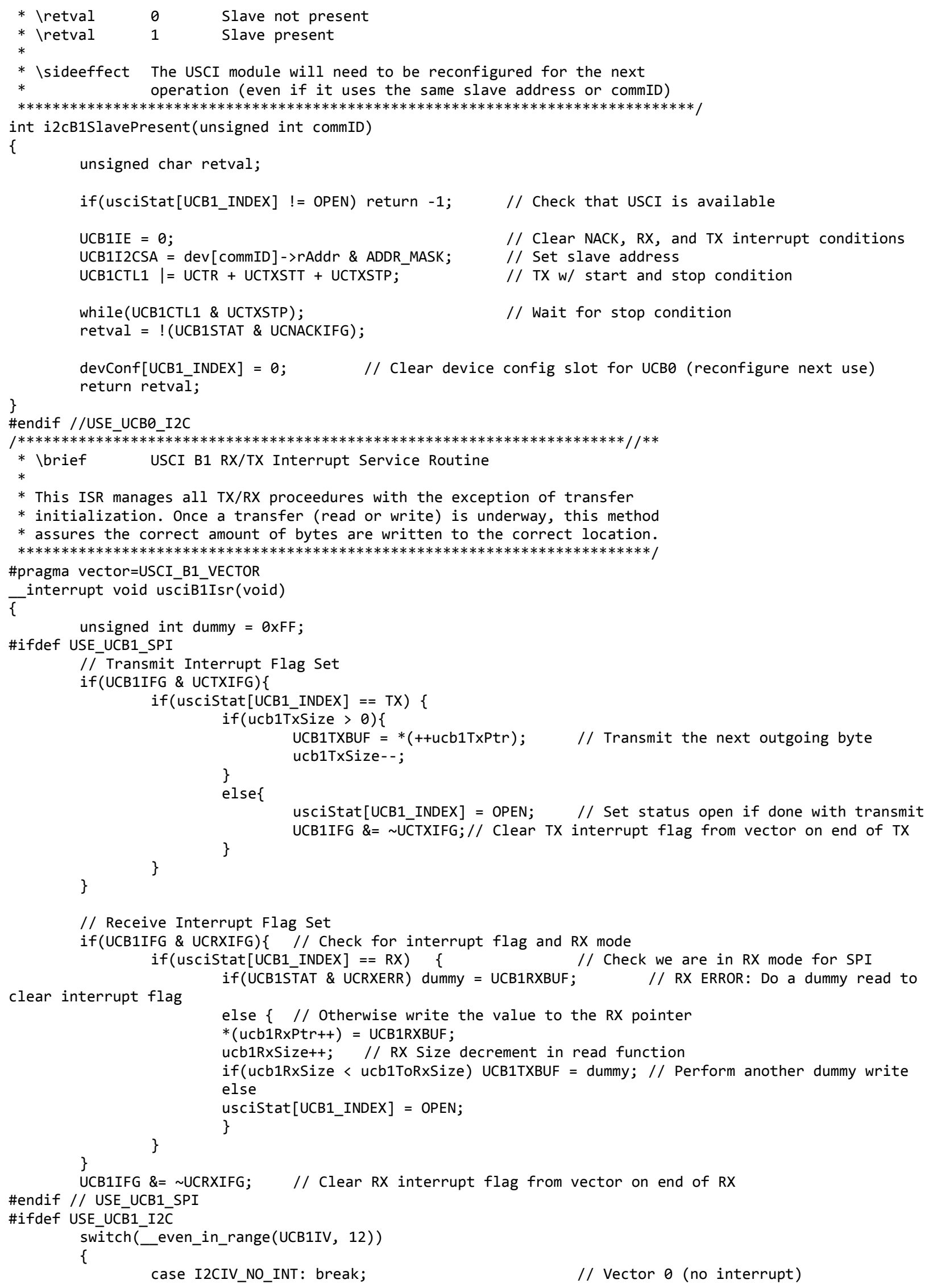




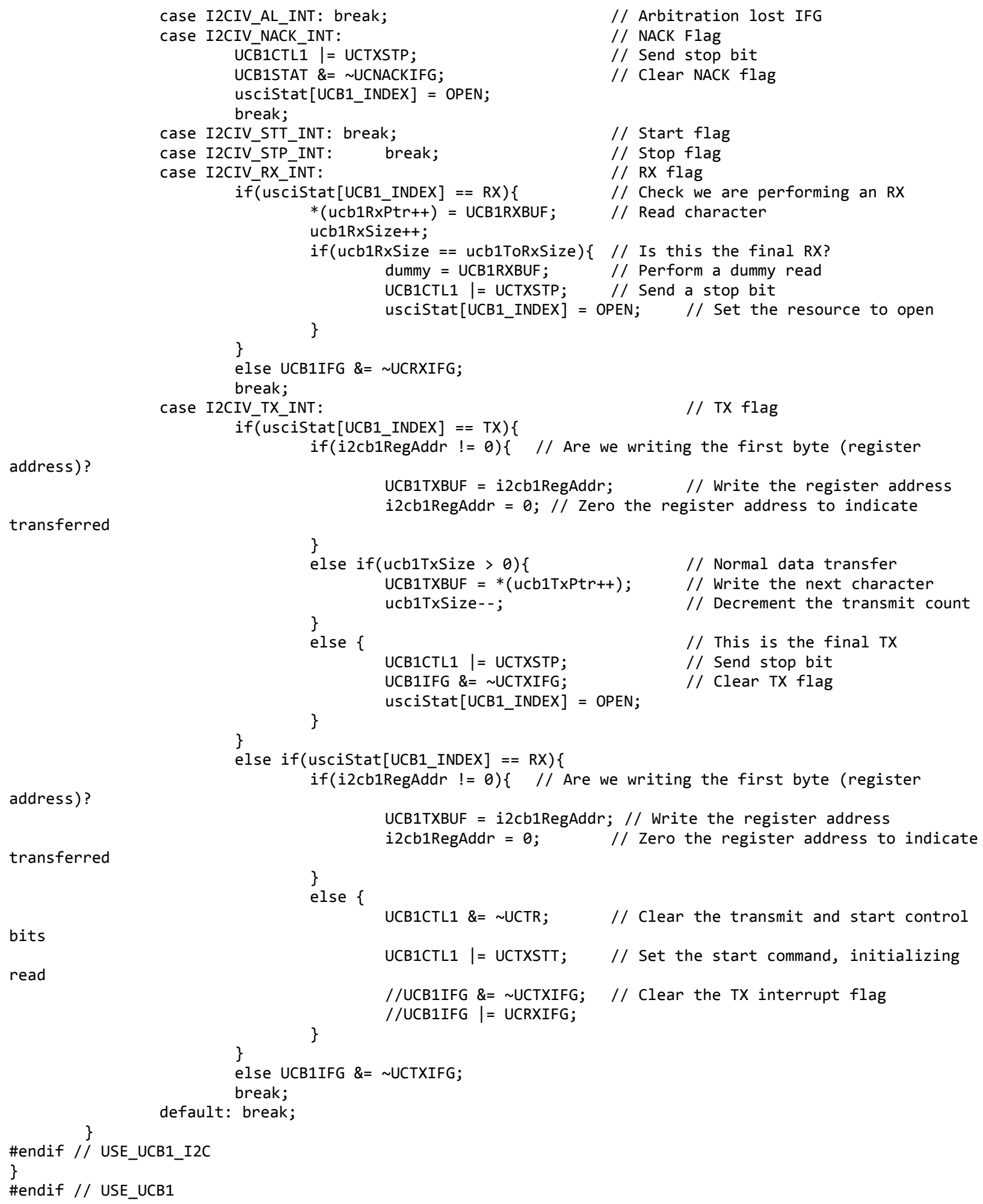

\section{Comm.h}

Header file affiliated with communications library 


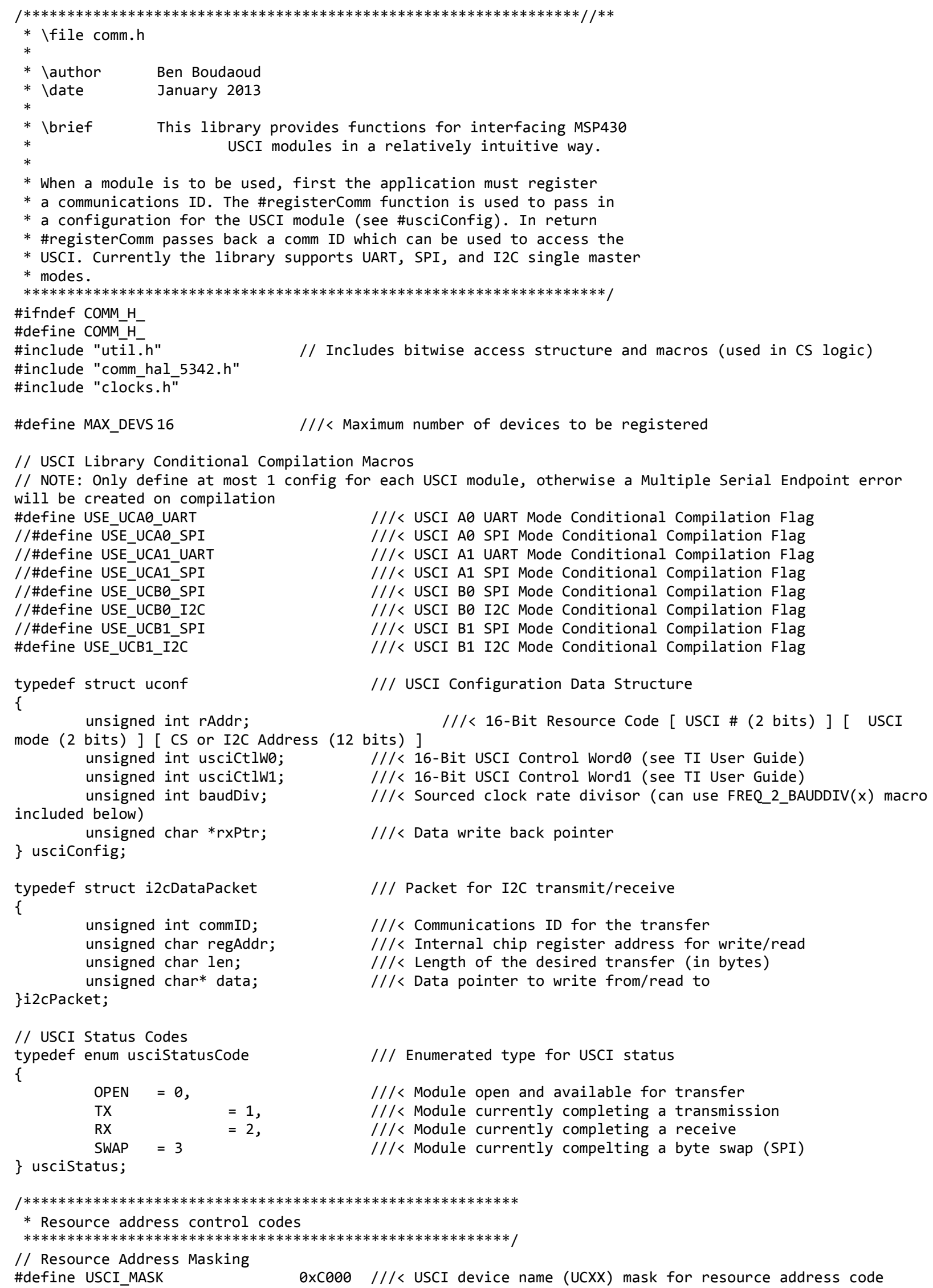


\#define MODE_MASK \#define UMODE_MASK \#define ADDR_MASK // Resource Codes \#define UCAO_RCODE \#define UCA1_RCODE \#define UCBO_RCODE \#define UCB1_RCODE \#define UART_MODE \#define SPI_MODE \#define I2C_MODE // Resource and Mode combo codes \#define UCAO_UART \#define UCAO_SPI \#define UCA1_UART \#define UCA1_SPI \#define UCBO_SPI \#define UCBO_I2C \#define UCB1_SPI \#define UCB1_I2C 0x3000 $/ / /<$ USCI mode (UART/SPI/I2C) name mask for resource address code 0xF000 $/ / /<$ USCI name/mode mask for resource address code 0x03FF $/ / /<$ USCI address mask for resource address code (max 10 bits)

0x0000 $/ / /<$ USCI AO resource code $0 \times 4000 / / /<$ USCI A1 resource code $0 \times 8000 / / /<$ USCI BO resource code 0xC000 $/ / /<$ USCI B1 resource code $0 \times 1000 / / /<$ UART mode code

$\theta \times 2000 / / /<$ SPI mode code $0 \times 3000 / / /<$ I2C mode code

UCAO RCODE + UART MODE $/ / /<$ Combined UCAO UART Mode Resource Code UCAO_RCODE + SPI_MODE $\quad / / /<$ Combined UCAO SPI Mode Resource Code UCA1_RCODE + UART_MODE $/ / /<$ Combined UCA1 UART Mode Resource Code UCA1_RCODE + SPI_MODE $\quad / / /<$ Combined UCA1 SPI Mode Resource Code UCBO_RCODE + SPI_MODE $\quad / / /<$ Combined UCBO SPI Mode Resource Code UCBO_RCODE + I2C_MODE $\quad / / /<$ Combined UCBO I2C Mode Resource Code UCB1_RCODE + SPI_MODE $\quad / / /<$ Combined UCB1 SPI Mode Resource Code UCB1_RCODE + I2C_MODE $/ / /<$ Combined UCB1 I2C Mode Resource Code

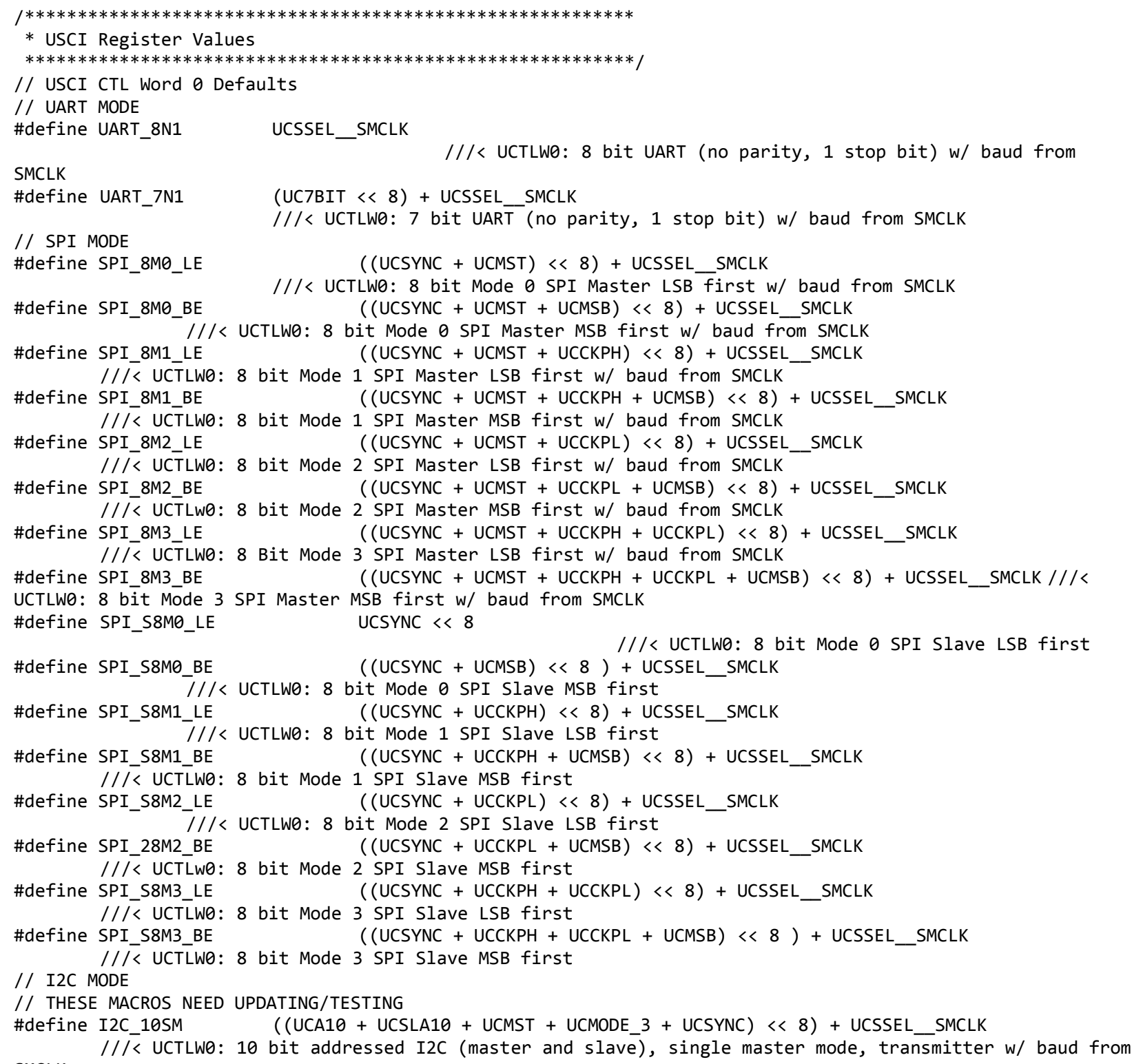
SMCLK 
\#define I2C_7SM ((UCMST + UCMODE_3 + UCSYNC) $<<8)+$ UCSSEL_SMCLK

$/ / \bar{K}$ UCTLWO: 7 bit addressed I2C (master and slave), single master mode, receiver $W /$ baud from

SMCLK

// I2C Interrupt Vector Definitions

\#define I2CIV_NO_INT 0

\#define I2CIV_AL_INT 2

\#define I2CIV_NACK_INT 4

\#define I2CIV_STT_INT 6

\#define I2CIV_STP_INT 8

\#define I2CIV_RX_INT 10

\#define I2CIV_TX_INT 12

$/ / /<$ I2C no interrupt source

$/ / /<$ I2C arbitration lost interrupt flag

$/ / /<$ I2C NACK interrupt flag

$/ / /<$ I2C start condition flag

$/ / /<$ I2C stop condition flag

$/ / /<$ I2C receive interrupt flag

// USCI CTL Work 1 Defaults

\#define DEF_CTLW1

$2 \quad / / /<I 2 C$ transmit interrupt flag

// USCI Baud Rate Defaults

\#define UCLK_FREQ

UART (from timing.h)]

\#define UBR_DIV $(x)$

timing.h)

0x0003 ///< CTLW1: 200ns deglitch time

// Resource config buffer index

\#define UCAO_INDEX 0

\#define UCA1_INDEX 1

\#define UCBO_INDEX 2

\#define UCB1_INDEX 3

SMCLK_FREQ $\quad / / /$ USCI Clock Rate [use SMCLK to source our

UCLK_FREQ/x $\quad / / /<$ Baud rate frequency to divisor macro (uses

// Read/Write Routine Return Codes

\#define USCI_CONF_ERROR - 2

\#define USCI_BUSY_ERROR

\#define USCI_SUCCËSS 1

$/ / /<$ USCI AO shared buffer index

$/ / /<$ USCI A1 shared buffer index

$/ / /<$ USCI BO shared buffer index

$/ / /<$ USCI B1 shared buffer index

// Time to start condition (in cycles) macro

\#define MAX_STT_WAIT 10000

$/ / /<$ USCI configuration error return code

$/ / /<$ USCI busy error return code

$/ / /<\mathrm{TX} / \mathrm{RX}$ success return code

// App. registration function prototype

int registerComm(usciConfig *conf);

/ $* * * * * * * * * * * * * * * * * * * * * * * * * * * * * * * * * * * * * * * * * * * * * * * * * * * * * * * * * * * * * * * * * * * * * * * * * * *$

* UCAO Macro Logic

$* * * * * * * * * * * * * * * * * * * * *$

// Basic function prototypes

void confUCA0(unsigned int commID);

void resetUCAO(unsigned int commID);

unsigned int getUCAORxSize(void);

unsigned char getUCAOStat(void);

void setUCAOBaud(unsigned int baudDiv, unsigned int commID);

/ $* * * * * * * * * * * * * * * * * * * * * * * * *$ UCAO UART MODE $* * * * * * * * * * * * * * * * * * * * * * * * * * * * * * * *$ /

\#ifdef USE_UCAO_UART

// Function prototypes

int uartAOWrite(unsigned char* data, unsigned int len, unsigned int commID);

int uartAORead(unsigned int len, unsigned int commID);

// Other useful macros

\#define USE_UCAO /// U UCAO Active Definition

// Multiple endpoint config detection

\#ifdef USE_UCAO_SPI

\#error Multiple Serial Endpoint Configuration on USCI AO

\#endif // USE_UCAO_UART and USE_UCAO_SPI

\#endif

/ $* * * * * * * * * * * * * * * * * * * * * * * * *$ UCAO SPI MODE $* * * * * * * * * * * * * * * * * * * * * * * * * * * * * * * * /$

\#ifdef USE_UCAO_SPI

// Function prototypes

int spiAOWrite (unsigned char* data, unsigned int len, unsigned int commID);

int spiAORead(unsigned int len, unsigned int commID);

unsigned char spiAOSwap(unsigned char byte, unsigned int commID);

// Multiple Endpoint Config Compiler Error

\#define USE_UCAO///< USCI AO Active Definition

\#ifdef USE_UCCAO_UART

\#error Multiple Serial Endpoint Configuration on USCI AO

\#endif // USE_UCAO_UART AND USE_UCAO_SPI 


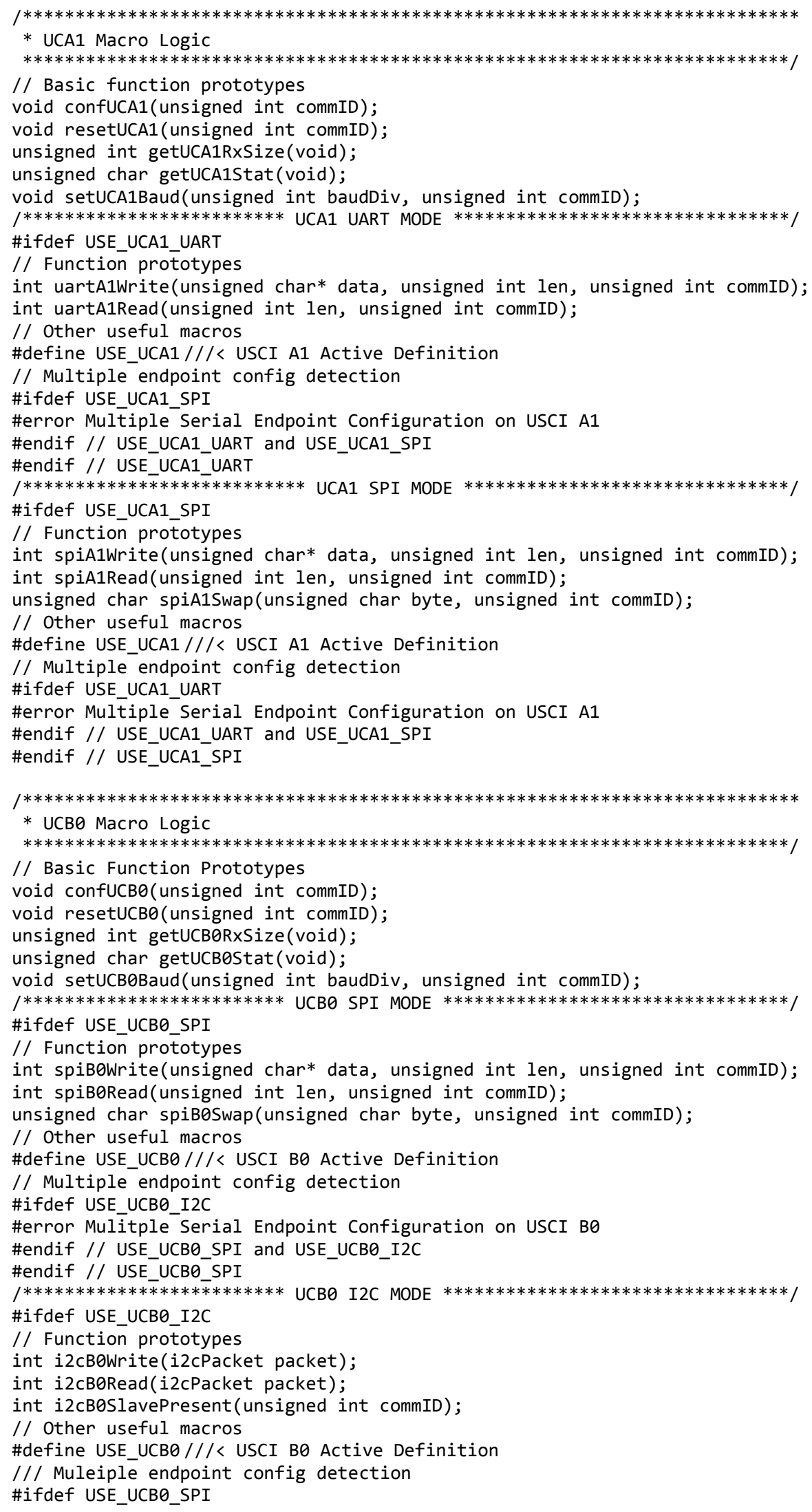


\#error Multiple Serial Endpoint Configuration on USCI BO

\#endif // USE_UCBO_SPI and USE_UCBO_I2C

\#endif // USE_UCBO_I2C

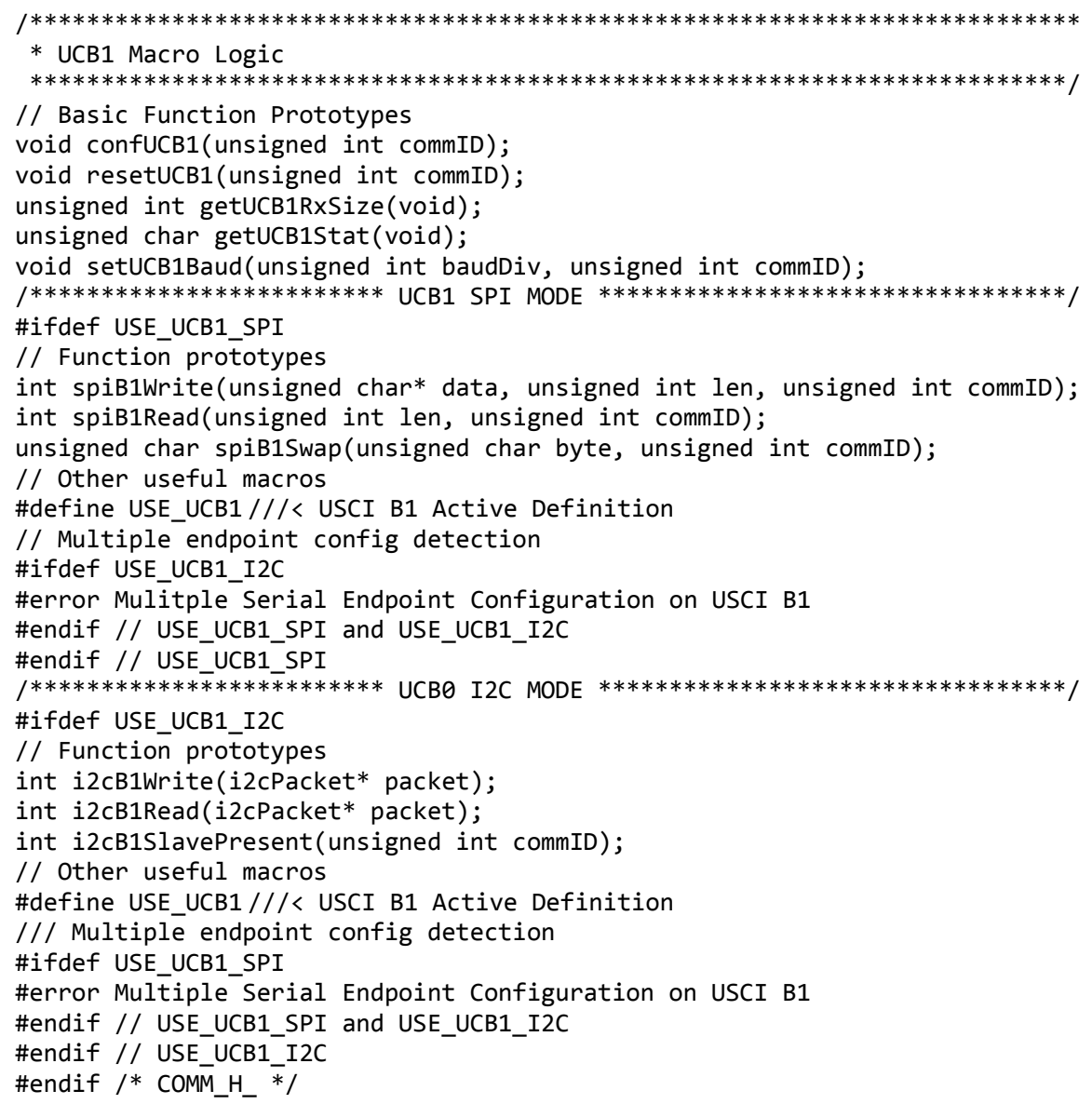

\section{Comm_hal_5342.h}

MSP430F5342-specific hardware definitions for use with the comm.c/h libraries

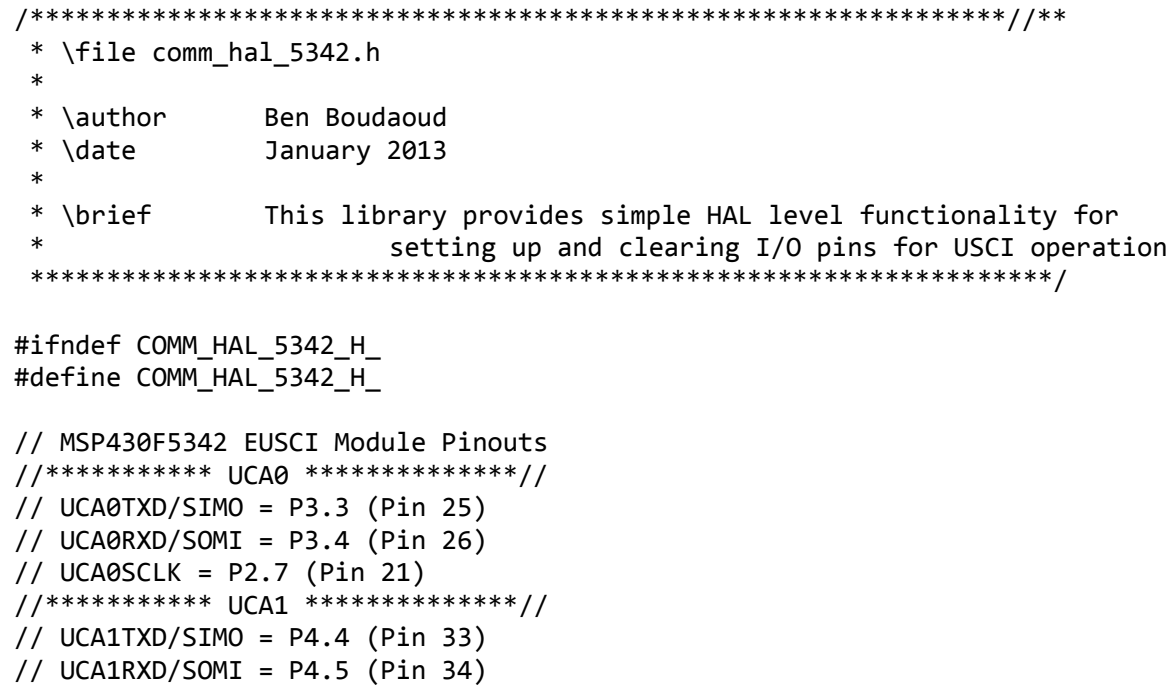




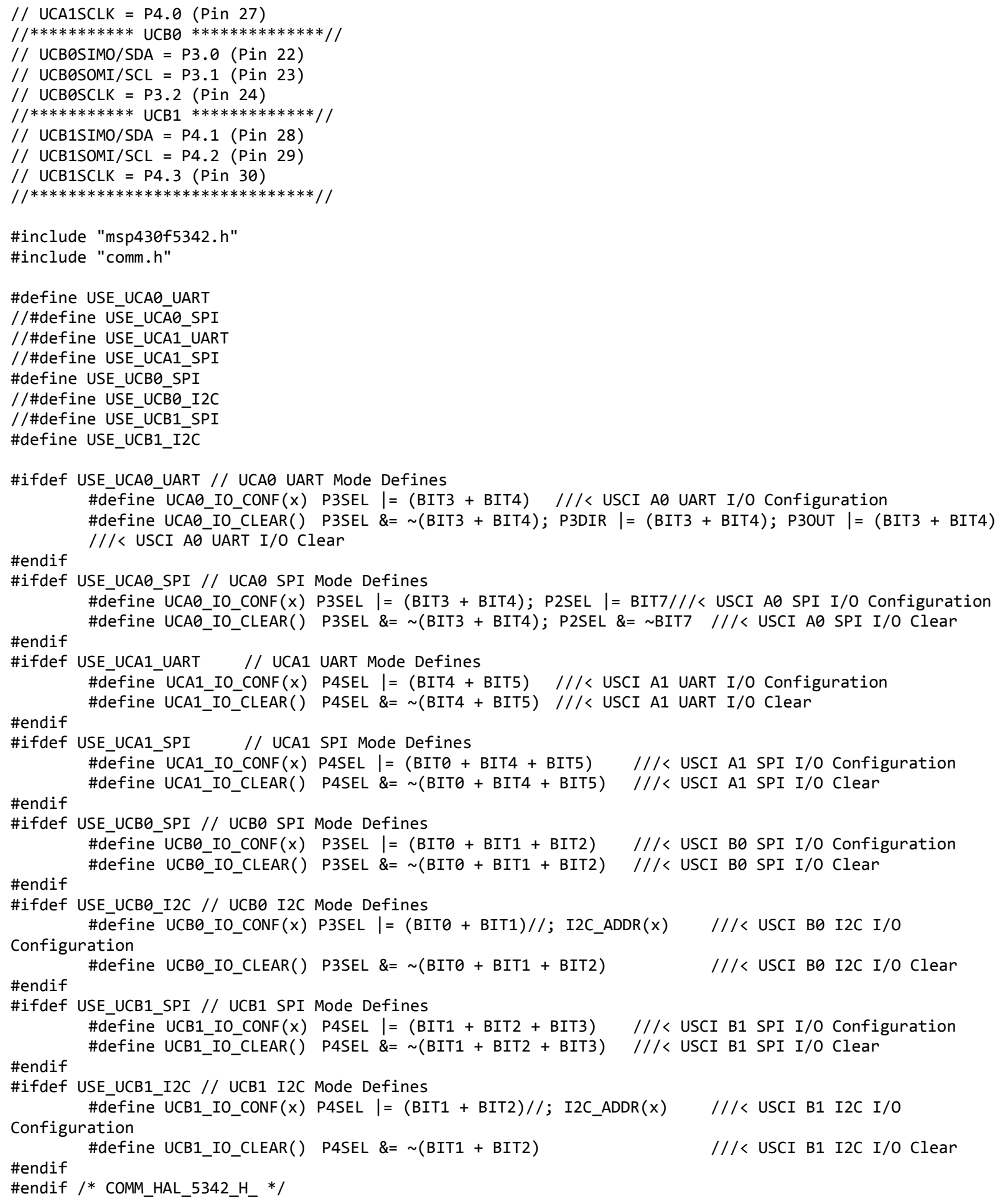

\section{Command.c}

System command interface for use on top of the FT232 code created to support UART-to-USB communication 


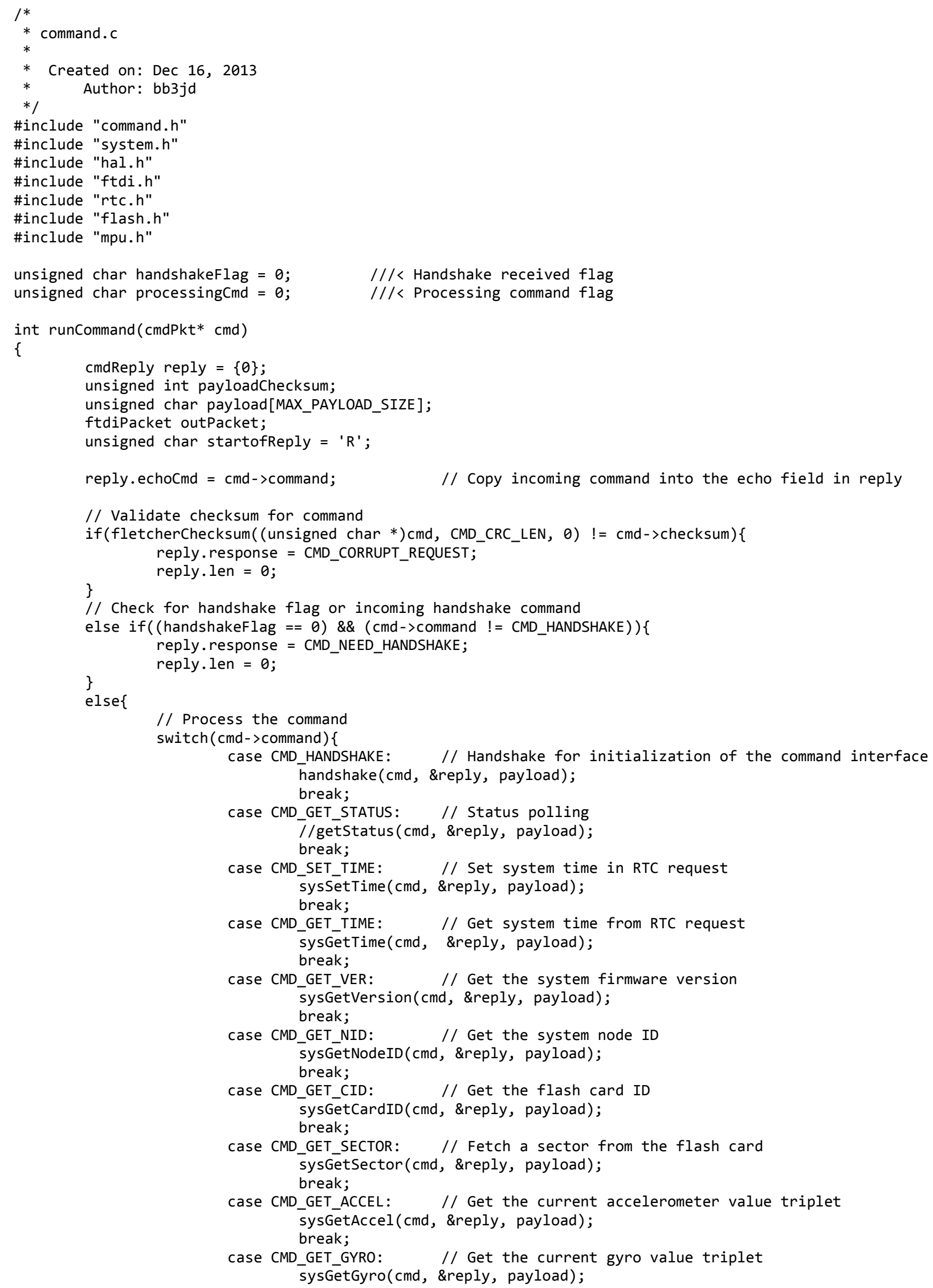


break;

case CMD_SET_SR: // Set the system sampling rate

sysSetSamplingRate(cmd, \&reply, payload);

break;

case CMD SET DCE :

sysSetDCE(cmd, \&reply, payload);

break;

case CMD_LED_ON: // Dummy command: turn on the LED

LED1_CONFIG( );

LED1_ON( );

reply. response $=$ CMD_SUCCESS;

reply.len = 0 ;

break;

case CMD_LED_OFF: // Dummy command: turn off the LED

LED1_CONFIG();

LED1_OFF();

reply. .response $=$ CMD_SUCCESS;

reply.len = 0 ;

default: break;

reply.response $=$ CMD_INVALID_COMMAND;

reply.len = 0 ;

\} break;

\}

if (reply.len > 0$)\{\quad / /$ If reply payload is populated payloadChecksum $=$ fletcherChecksum (payload, reply.len, $\theta) ; / /$ Perform checksum \} reply.len $+=$ sizeof(payloadChecksum);// Pack the checksum length into the reply header

reply. checksum $=$ fletcherChecksum $\left(\left(\right.\right.$ unsigned char $\left.{ }^{*}\right) \&$ reply, sizeof $($ reply $)$-sizeof $($ reply.checksum $)$,

$0)$; // Compute reply header chekcsum

// Send the start of reply

outPacket.data = \&startofReply;

outPacket.len $=1$;

ftdiWrite(outPacket);

// Send the reply header

outPacket.data $=$ (unsigned char $*$ )\&reply;

outPacket.len = sizeof (reply);

ftdiWrite(outPacket);

// Send the reply payload (if one exists)

if(reply.len > 0$)\{$ outPacket.data = payload;

outPacket.len = reply.len - sizeof(payloadChecksum);

ftdiWrite(outPacket);

// Send the reply payload checksum at the end of the payload

outPacket.data $=\left(\right.$ unsigned char $\left.^{*}\right)($ \&payloadChecksum $)$;

outPacket.len = sizeof (payloadChecksum);

\} ftdiWrite(outPacket);

\}

processingCmd = 0 ;

// Clear processing command flag (for incoming parser management) return CMD_SUCCESS;

$/ * * * * * * * * * * * * * * * * * * * * * * * * * * * * * * * * * * * * * * * * * * * * * * * * * * * * * * * * * * * * * * * * * * * * * * * * * * * * / / * *$

* Ibrief "Handshake" with the node to unlock access to the other commands

*

* Iparam[in] cmd Pointer to the command passed into the run function

* Iparam[out] reply Pointer to the reply packet to be sent in response

* Iparam[out] payload Pointer to the data payload to be sent with the reply

$* * * * * * * * * * * * * * * * * * * * * * * * * * * * * * * * * * * * * * * * * * * * * * * * * * * * * * * * * * * * * * * * * * * * * * * * * * * * * *)$

void handshake (cmdPkt* cmd, cmdReply* reply, unsigned char* payload) \{

handshakeFlag = 1 ;

reply->len = 0 ; 


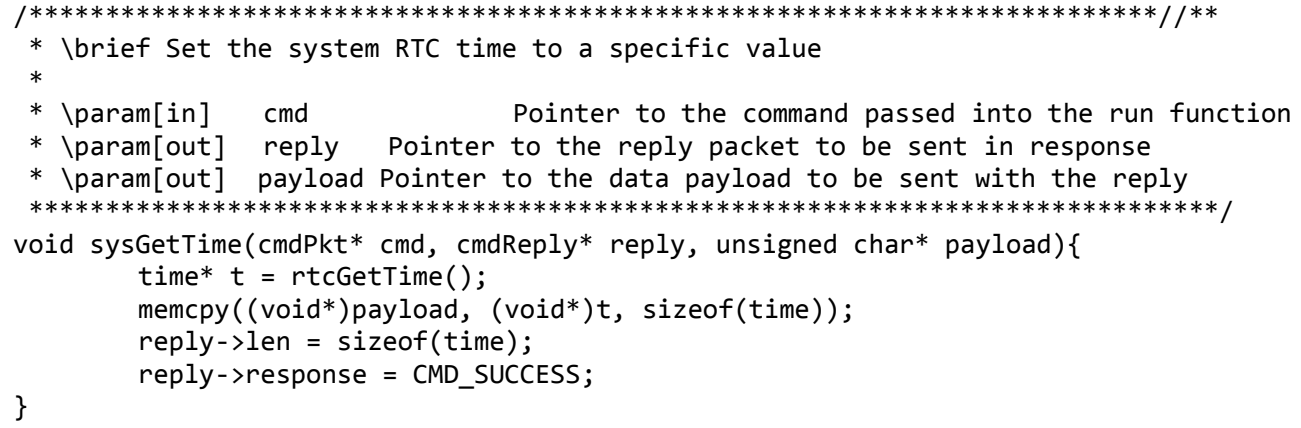




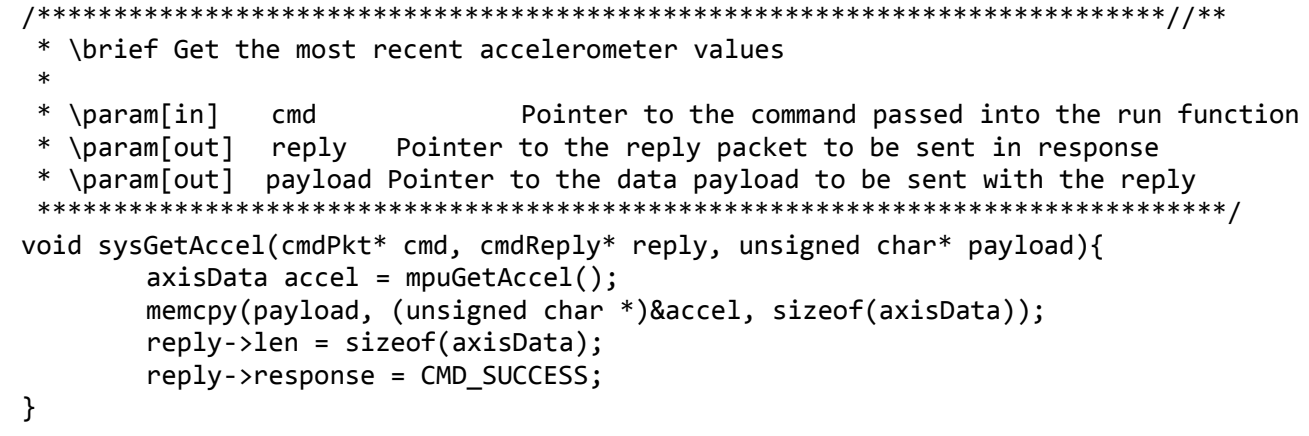




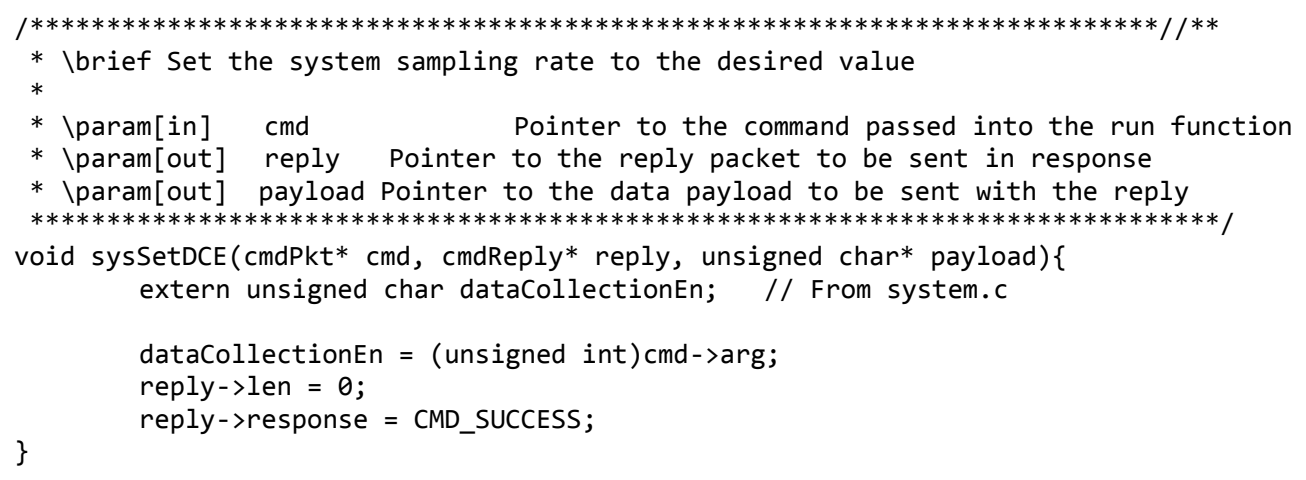

\section{Command.h}

Affiliated command interface header file

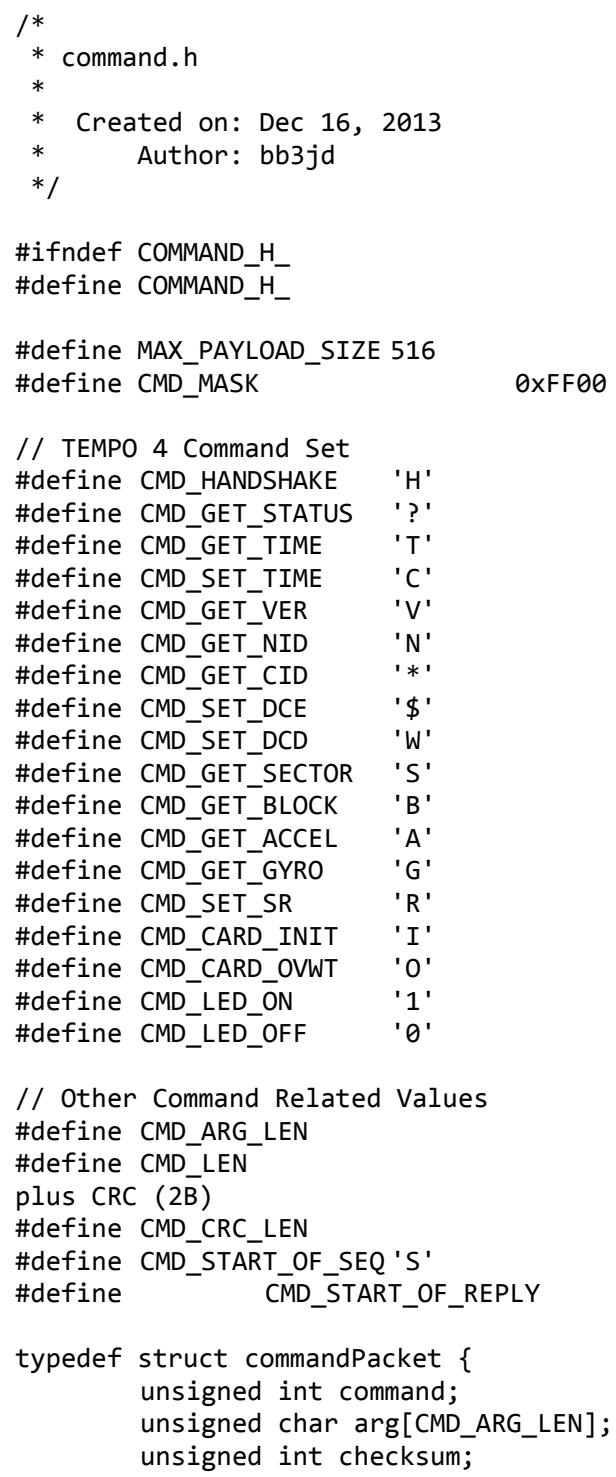

// Other Command Related Values \#define CMD ARG LEN

\#define CMD_LEN

plus CRC (2B)

\#define CMD_CRC_LEN

\#define CMD START OF SEQ 'S'

\#define CMD_START_OF_REPLY

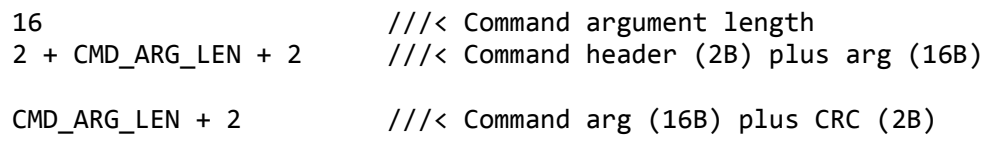

/// TEMPO 4 Incoming Command Structure

$/ / /<$ The command to be processed

$/ / /<$ The argument to the command

$/ / /<$ Checksum for validity purposes 
\} cmdPkt;

// Command Response Codes \#define CMD_CORRUPT_REQUEST

\#define CMD NEED HANDSHAKE

\#define CMD_INVALID_COMMAND

command set

\#define CMD_FAIL_READ_ONLY

\#define CMD_FAIL_GENERAL

\#define CMD_BAD_ARG

\#define CMD SUCCESS

typedef struct replyHeader \{ unsigned int echocmd; unsigned int response; unsigned int len;

\} cmaReply; unsigned int checksum;
$/ / /<$ The command received did not pass checksum $/ / /<$ No handshake has been passed to this node $/ / /<$ No such command exists in the current

$/ / /<$ The device is in read-only mode

$/ / /<$ General failure case (catch all)

5

$6 \quad / / /<$ Invalid argument failure

$/ / /<$ Command processed successfully

/// TEMPO 4 Outgoing Response Structure

$/ / /<$ Command echo field

$/ / /<$ Command response code field

$/ / /<$ Command response length field

$/ / /<$ Command response checksum field

// Function Prototypes

int runCommand (cmdPkt $\left.{ }^{*} \mathrm{cmd}\right)$;

$/ * * * * * * * * * * * * * * * * * * * * * * * * * * * * * * * * * * * * * * * * * * * * * * * * * * * * * * * * * * * * * * * * * * * * * * * * * * * * * * * / /$ / *

* \brief "Handshake" with the node to unlock access to the other commands

* Iparam[in] cmd Pointer to the command passed into the run function

* Iparam[out] reply Pointer to the reply packet to be sent in response

* Iparam[out] payload Pointer to the data payload to be sent with the reply

$* * * * * * * * * * * * * * * * * * * * * * * * * * * * * * * * * * * * * * * * * * * * * * * * * * * * * * * * * * * * * * * * * * * * * * * * * * * * * * * * * * *)$

void handshake (cmdPkt* cmd, cmdReply* reply, unsigned char* payload);

$/ * * * * * * * * * * * * * * * * * * * * * * * * * * * * * * * * * * * * * * * * * * * * * * * * * * * * * * * * * * * * * * * * * * * * * * * * * * * * * / / / * *$

* \brief Set the system RTC time to a specific value

* Iparam[in] cmd Pointer to the command passed into the run function

* Iparam[out] reply Pointer to the reply packet to be sent in response

* Iparam[out] payload Pointer to the data payload to be sent with the reply

$* * * * * * * * * * * * * * * * * * * * * * * * * * * * * * * * * * * * * * * * * * * * * * * * * * * * * * * * * * * * * * * * * * * * * * * * * * * * * * * * * * *$ /

void sysGetTime(cmdPkt* cmd, cmdReply* reply, unsigned char* payload);

$/ * * * * * * * * * * * * * * * * * * * * * * * * * * * * * * * * * * * * * * * * * * * * * * * * * * * * * * * * * * * * * * * * * * * * * * * * * * * * / /$ / $*$

* \brief Get the system RTC time and return it in the payload

* Iparam[in] cmd Pointer to the command passed into the run function

* Iparam[out] reply Pointer to the reply packet to be sent in response

* Iparam[out] payload Pointer to the data payload to be sent with the reply

$* * * * * * * * * * * * * * * * * * * * * * * * * * * * * * * * * * * * * * * * * * * * * * * * * * * * * * * * * * * * * * * * * * * * * * * * * * * * * * * * * * *)$

void sysSetTime(cmdPkt* cmd, cmdReply* reply, unsigned char* payload);

$/ * * * * * * * * * * * * * * * * * * * * * * * * * * * * * * * * * * * * * * * * * * * * * * * * * * * * * * * * * * * * * * * * * * * * * * * * * * * * * / / / * *$

* $\backslash$ brief Get the system firmware version

* Iparam[in] cmd Pointer to the command passed into the run function

* Iparam[out] reply Pointer to the reply packet to be sent in response

* Iparam[out] payload Pointer to the data payload to be sent with the reply

$* * * * * * * * * * * * * * * * * * * * * * * * * * * * * * * * * * * * * * * * * * * * * * * * * * * * * * * * * * * * * * * * * * * * * * * * * * * * * * *$ /

void sysGetVersion(cmdPkt* cmd, cmdReply* reply, unsigned char* payload);

$/ * * * * * * * * * * * * * * * * * * * * * * * * * * * * * * * * * * * * * * * * * * * * * * * * * * * * * * * * * * * * * * * * * * * * * * * * * * * / /$ / $*$

* $\backslash$ brief Get the node identification number

* Iparam[in] cmd Pointer to the command passed into the run function

* Iparam[out] reply Pointer to the reply packet to be sent in response

* Iparam[out] payload Pointer to the data payload to be sent with the reply

$* * * * * * * * * * * * * * * * * * * * * * * * * * * * * * * * * * * * * * * * * * * * * * * * * * * * * * * * * * * * * * * * * * * * * * * * * * * * * * *)$

void sysGetNodeID(cmdPkt* cmd, cmdReply* reply, unsigned char* payload);

$/ * * * * * * * * * * * * * * * * * * * * * * * * * * * * * * * * * * * * * * * * * * * * * * * * * * * * * * * * * * * * * * * * * * * * * * * * * * / / * *$

* \brief Get the card identification number

* Iparam[in] cmd Pointer to the command passed into the run function

* Iparam[out] reply Pointer to the reply packet to be sent in response

* Iparam[out] payload Pointer to the data payload to be sent with the reply

$* * * * * * * * * * * * * * * * * * * * * * * * * * * * * * * * * * * * * * * * * * * * * * * * * * * * * * * * * * * * * * * * * * * * * * * * * * * * * * *)$

void sysGetCardID(cmdPkt* cmd, cmdReply* reply, unsigned char* payload); 


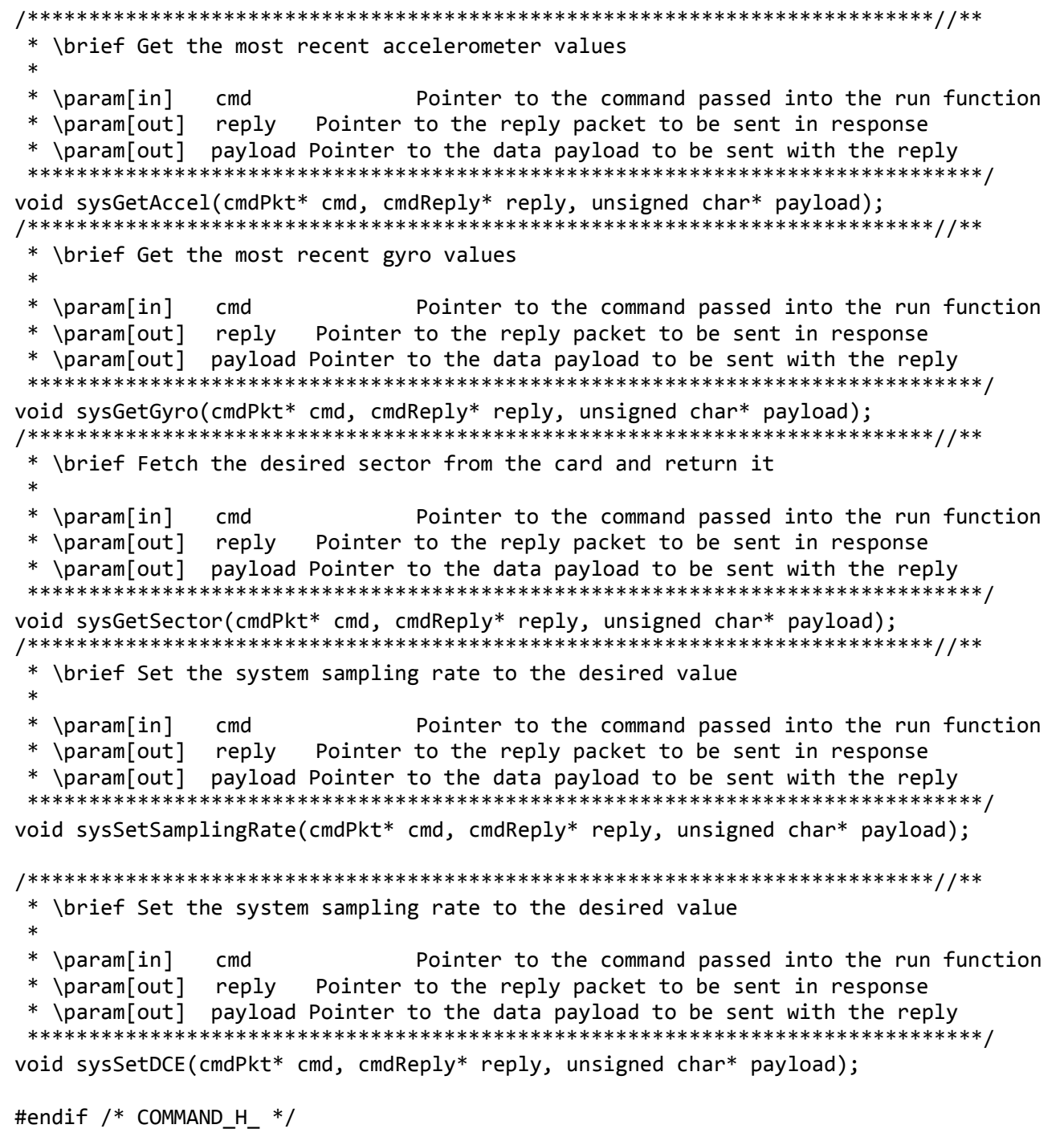

\section{Filesystem.c}

Implementation of the TEMPO 4 file system

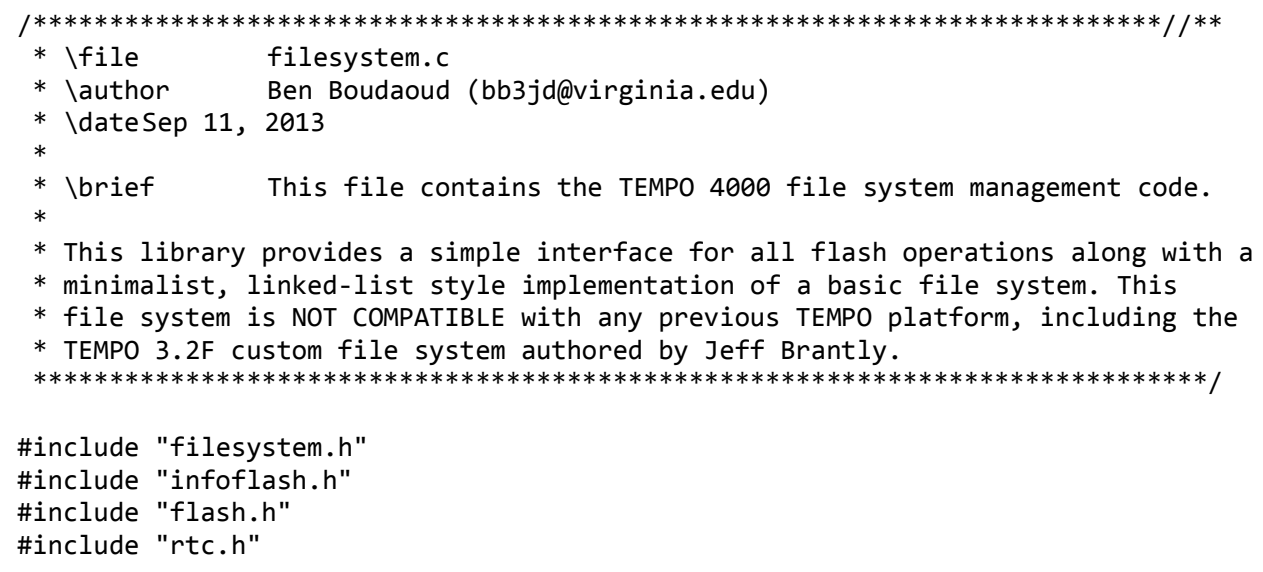


\#include 〈string.h>

cardInfo cardData;
Node information data structure
sessInfo sessData;
Session information data structure
$\begin{aligned} & \text { unsigned char dBuff[CARD_BUFFER_SIZE]; } \\ & \text { unsigned int putIndex, getIndex = } 0 ;\end{aligned} \quad / / / / /$ Card data buffer
unsigned int bytesToWrite $=0 ;$
card data buffer

unsigned long currSessSector $=0$;

$/ / /<$ Holds the index of the current sessions info sector unsigned long currsector $=0$;

$/ / /<$ Tracks the current flash card sector

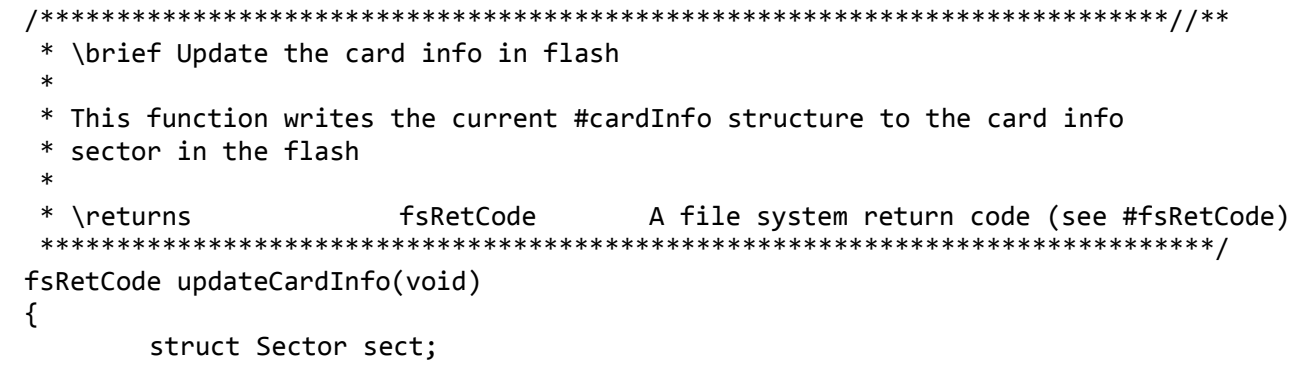




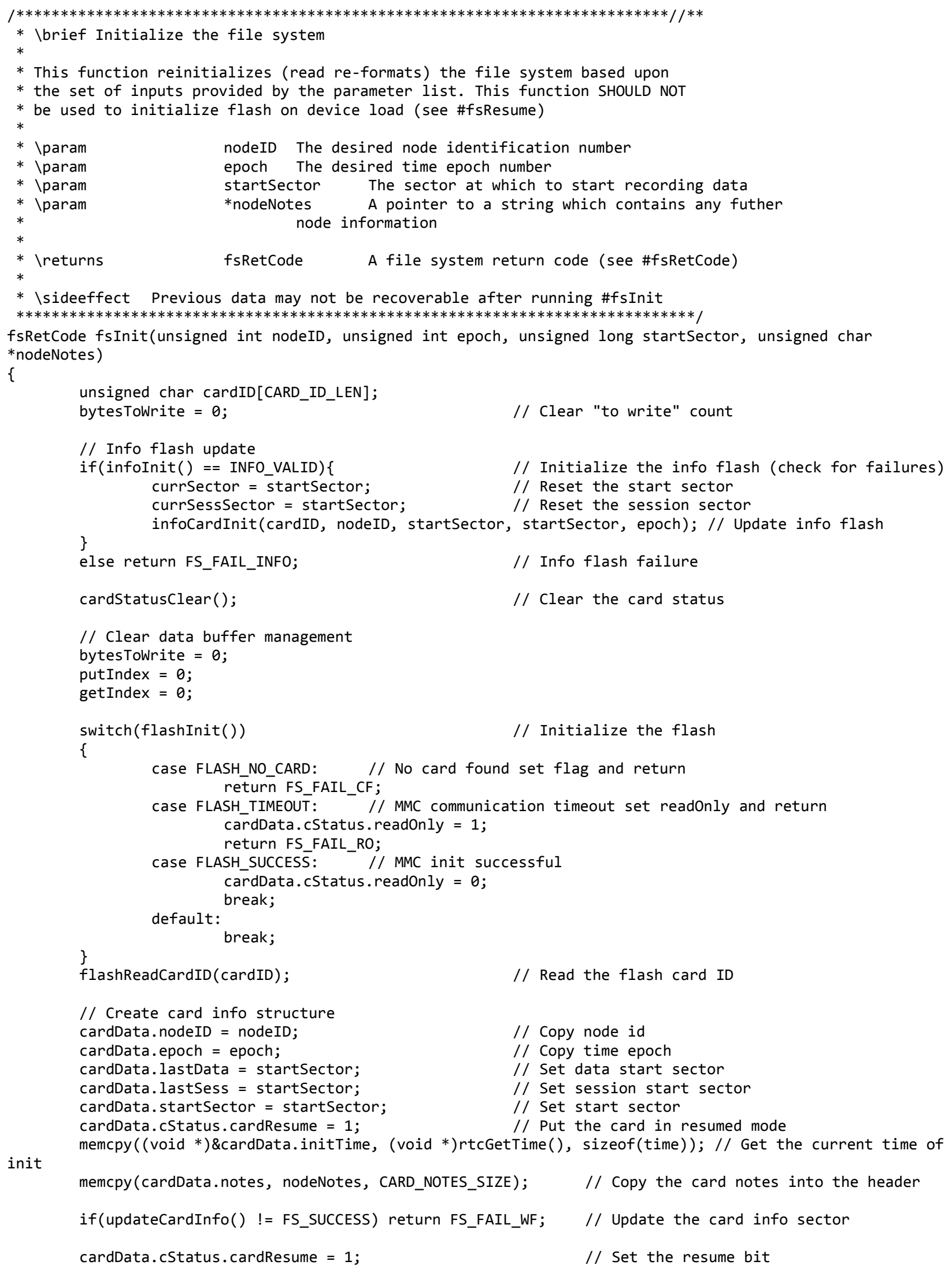




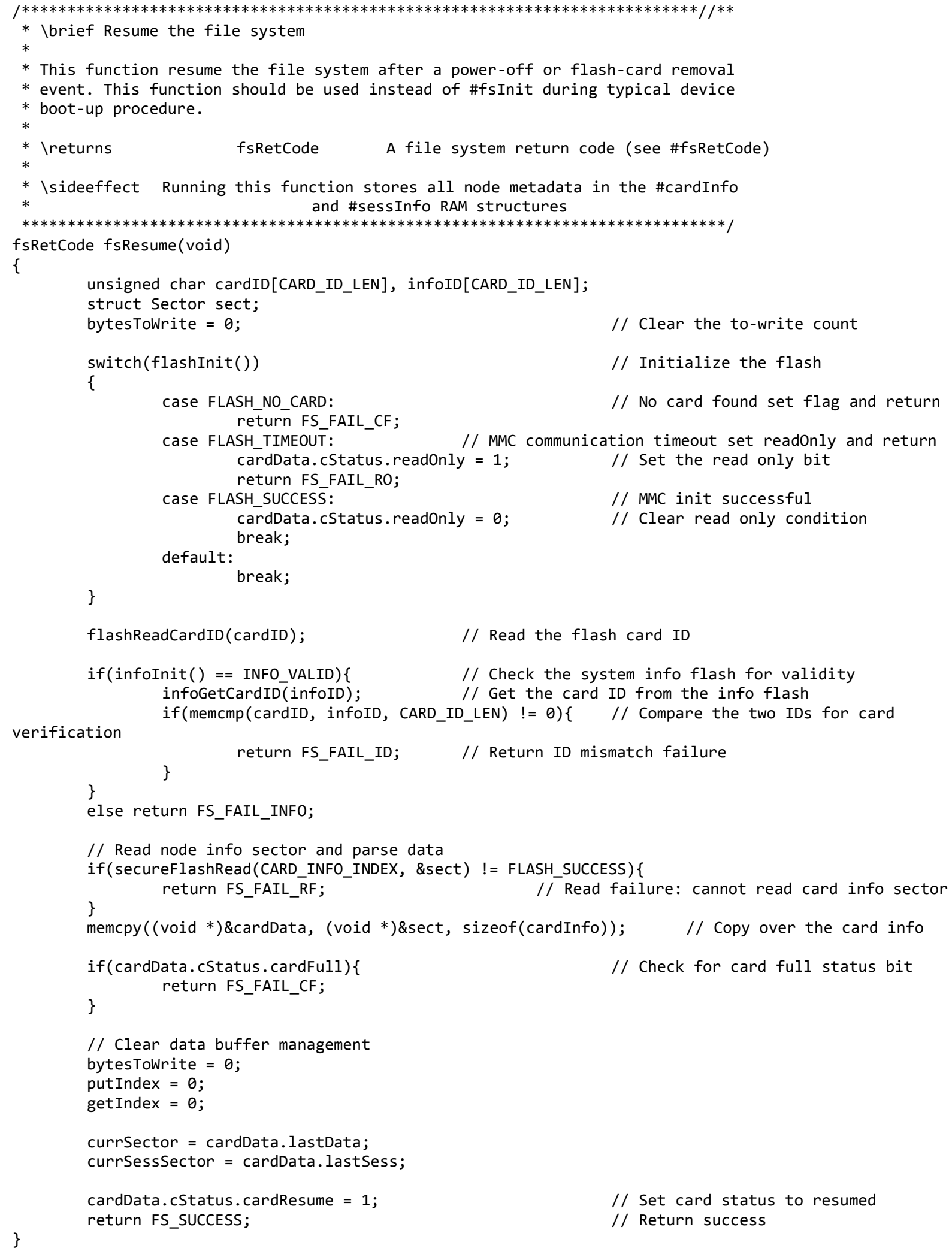


* \brief Start a new session

* This function begins a new data session in the file system. This function

* should only be called when no other session is in progress, and after the

* file system has been successfully resumed or initialized.

* Iparam SR

* Iparam axis

* Iparam startTime

$*$

$*$

* \retval

* Isideeffect Sets the session in progress (SIP) flag to prevent multiple

* sessions being open at once.

$* * * * * * * * * * * * * * * * * * * * * * * * * * * * * * * * * * * * * * * * * * * * * * * * * * * * * * * * * * * * * * * * * * * * * * * * * * * * * * *) /$

fsRetCode fsStartSession(unsigned int SR, axisCtrl axis, time* startTime)

\{

// Condition code checks

if (cardData.cStatus. readOnly $==1$ ) return FS_FAIL_RO;

// Check for read only condition

else if (cardData.cStatus.sessInProgress $==\overline{1}$ ) return FS_FAIL_SIP; // Check for another session in progress

else if (cardData.cStatus.cardResume $==1$ ) return FS_FAIL_NR; // Check for resume condition enabled else if (cardData.cStatus.dataCollectionEn = = 0 ) return F $\bar{S}$ FAIL_DCE; // Check for data collection condition

else if(currSector $>=$ TOTAL_SECTORS || cardData.cStatus.cardFull) $\{\quad / /$ Check for card full

cardData.cStatus. cardFull = 1 ;

return FS_FAIL_CF;

\}

// Card full return code

// Previous session info update (write 1)

sessData. nextSessSector $=++$ currSector;

// Set the last sessions 'next' value to this session's

if(writeSessInfo(currSessSector) != FS_SUCCESS) return FS_FAIL_WF; // Update the old session

// Current session info update (write 2)

sessData. serial $=$ sessData. serial +1 ;

// Increment the session serial number

sessData. lastSessSector = currSessSector;

// Set the last start sector

sessData. nextSesssector = (unsigned int) $(-1)$;

// Set the next start sector to -1

sessData. length $=0$;

// Set the starting length to $\theta$

sessData. samplingRate $=\mathrm{SR}$;

// Set the sampling rate to the provided rate

sessData.axis = axis;

// Set the axis bit field to provided value

sessData.status = sess open;

// Set the session status to open

if $($ startTime $->$ mon $==0)\{$

// Check for null start time (month cannot be 0 )

memcpy $\left(\left(\operatorname{void}^{*}\right) \&\left(\operatorname{sessData}\right.\right.$. startTime), (void $\left.{ }^{*}\right)$ rtcGetTime(), sizeof(time)); // If needed

get a valid start time from the RTC

\}

else \{

start time object

memcpy $((\operatorname{void} *) \&($ sessData.startTime), (void *)\&startTime, sizeof(time)); // Copy over

\}

if(writeSessInfo(currSector) != FS_SUCCESS) return FS_FAIL_WF; // Set sector information and write sector to card

// Clear data buffer management

bytesToWrite $=0$;

putIndex $=0$;

getIndex $=0$; 


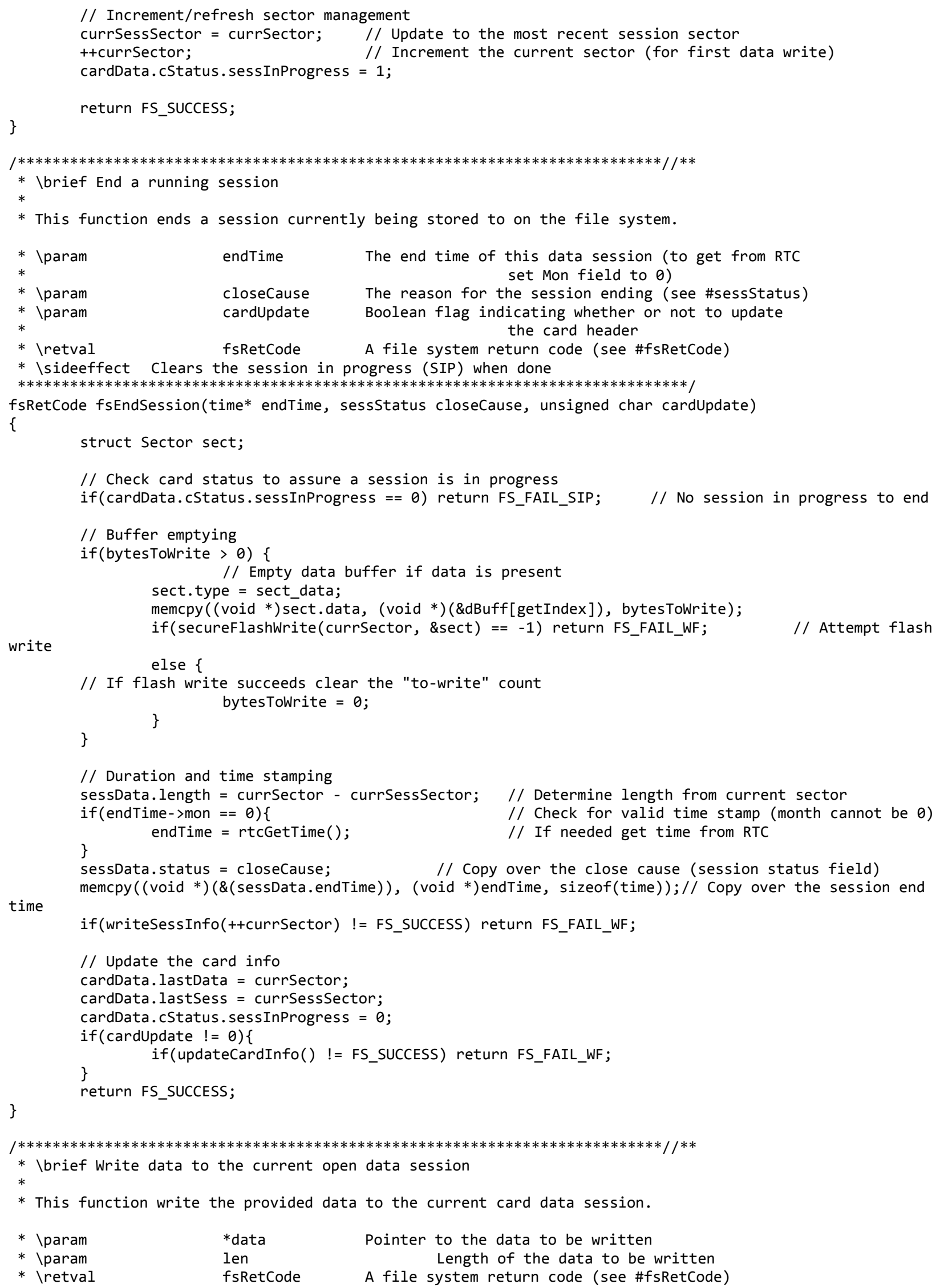




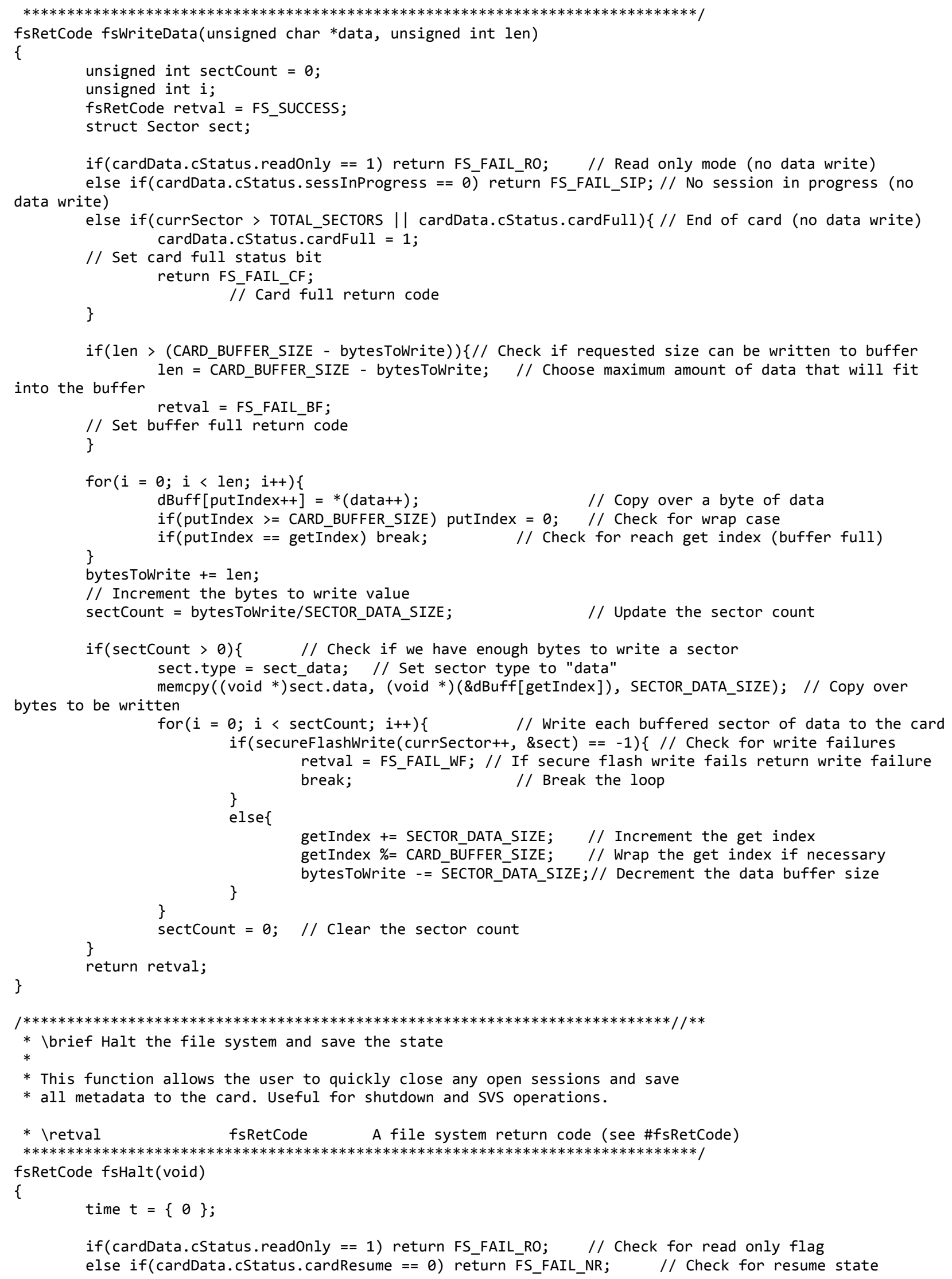




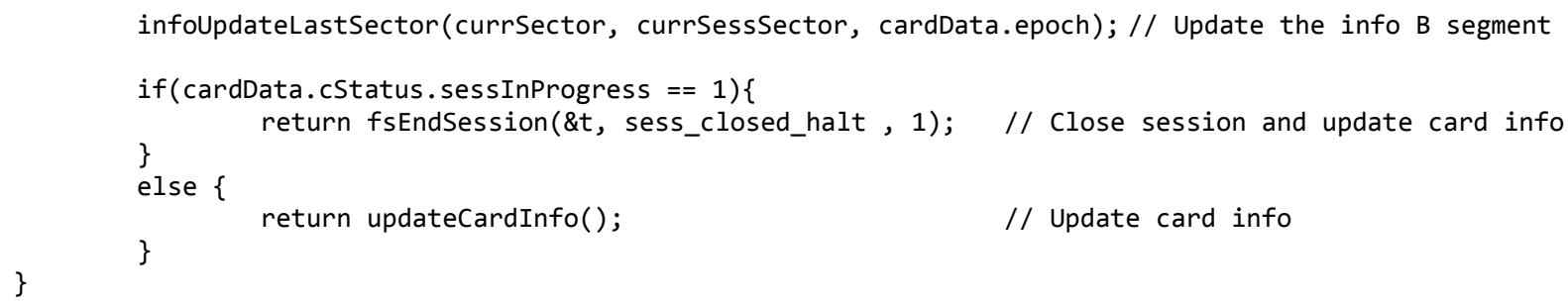

\section{Filesystem.h}

Affiliated file system header file

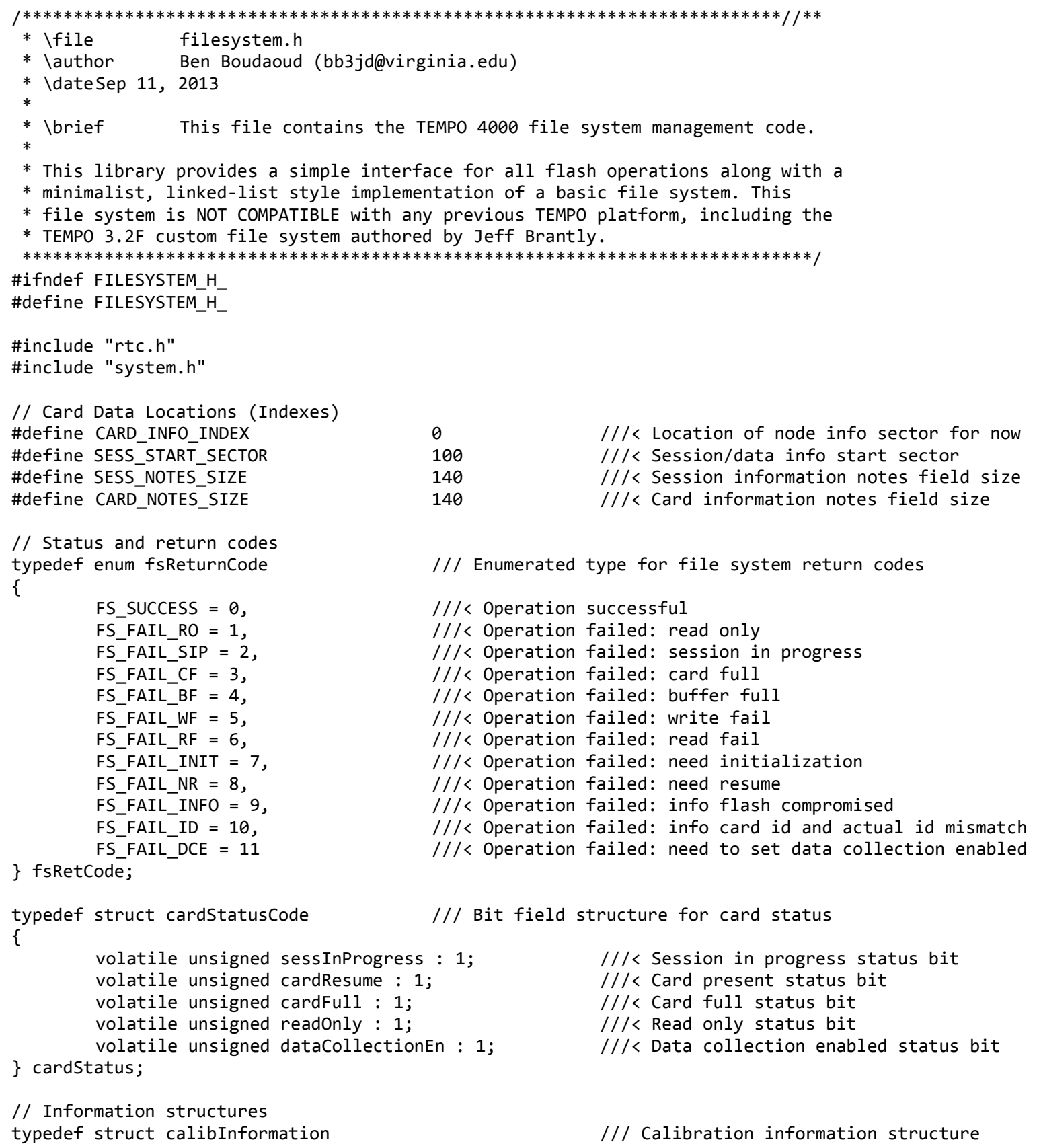

volatile unsigned dataCollectionEn : 1 ; $\quad / / /<$ Data collection enabled status bit

$/ / /<$ Session in progress status bit

$/ / /<$ Card present status bit

$1 / /<$ Card full status bit

$/ / /<$ Read only status bit

/// Calibration information structure 
unsigned int $\mathrm{x}$ _acc_offset; unsigned int $y$ _acc_offset; unsigned int z_acc_offset; unsigned int $x_{-}$acc_sens; unsigned int y_acc_sens; unsigned int z_acc_sens; unsigned int x_gyro_offset; unsigned int y_gyro_offset; unsigned int z_gyro_offset; unsigned int x_gyro_sens; unsigned int y_gyro_sens; unsigned int $z$ gyro sens;
$/ / /<X$ Accelerometer DC offset

$/ / /<Y$ Accelerometer DC offset

$/ / /<$ Z Accelerometer DC offset

$/ / /<\mathrm{X}$ Accelerometer AC sensitivity

$/ / /<Y$ Y Accelerometer DC sensitivity

$/ / /<$ Z Accelerometer DC sensitivity

$/ / /<X$ Gyro DC offset

$/ / /<Y$ Gyro DC offset

$/ / /<Z$ Gyro DC offset

$/ / /<X$ Gyro AC sensitivity

$/ / /<Y$ Gyro AC sensitivity

$/ / /<Z$ Gyro AC sensitivity

/// Iwarning Do not increase this structure beyond \#SECTOR_SIZE bytes! typedef struct cardInformation

/// Card information structure \{

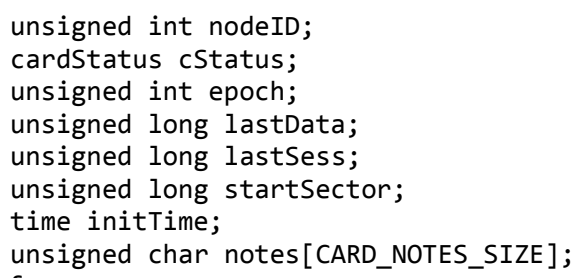

$/ / /<$ Node identification number

$/ / /<$ Card status indicator field

$/ / /<$ Time epoch number

$/ / /<$ Last session data sector index

$/ / /<$ Last session info sector index

$/ / /<$ Start sector index

$/ / /<$ Card initialization time

$/ / /<$ Card information string

/// Iwarning Do not increase this structure beyond \#SECTOR_SIZE bytes! typedef struct sessionInformation

/// Session information \{

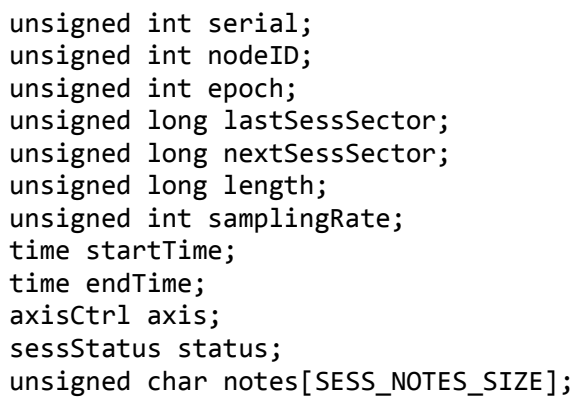


* This function resets the card status field to whatever should be the default

* value/set of values.

* Ireturns fsRetCode A file system return code (see \#fsRetCode)

$* * * * * * * * * * * * * * * * * * * * * * * * * * * * * * * * * * * * * * * * * * * * * * * * * * * * * * * * * * * * * * * * * * * * * * * * * * * * * * * *)$

inline void cardStatusClear(void);

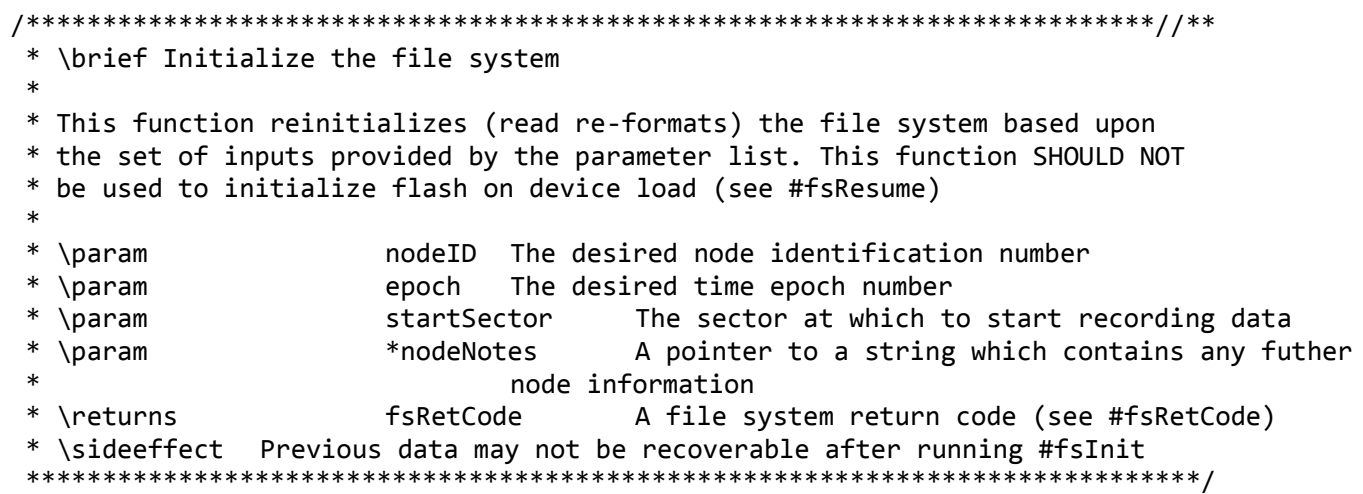

fsRetCode fsInit(unsigned int nodeID, unsigned int epoch, unsigned long startSector, unsigned char *nodeNotes);
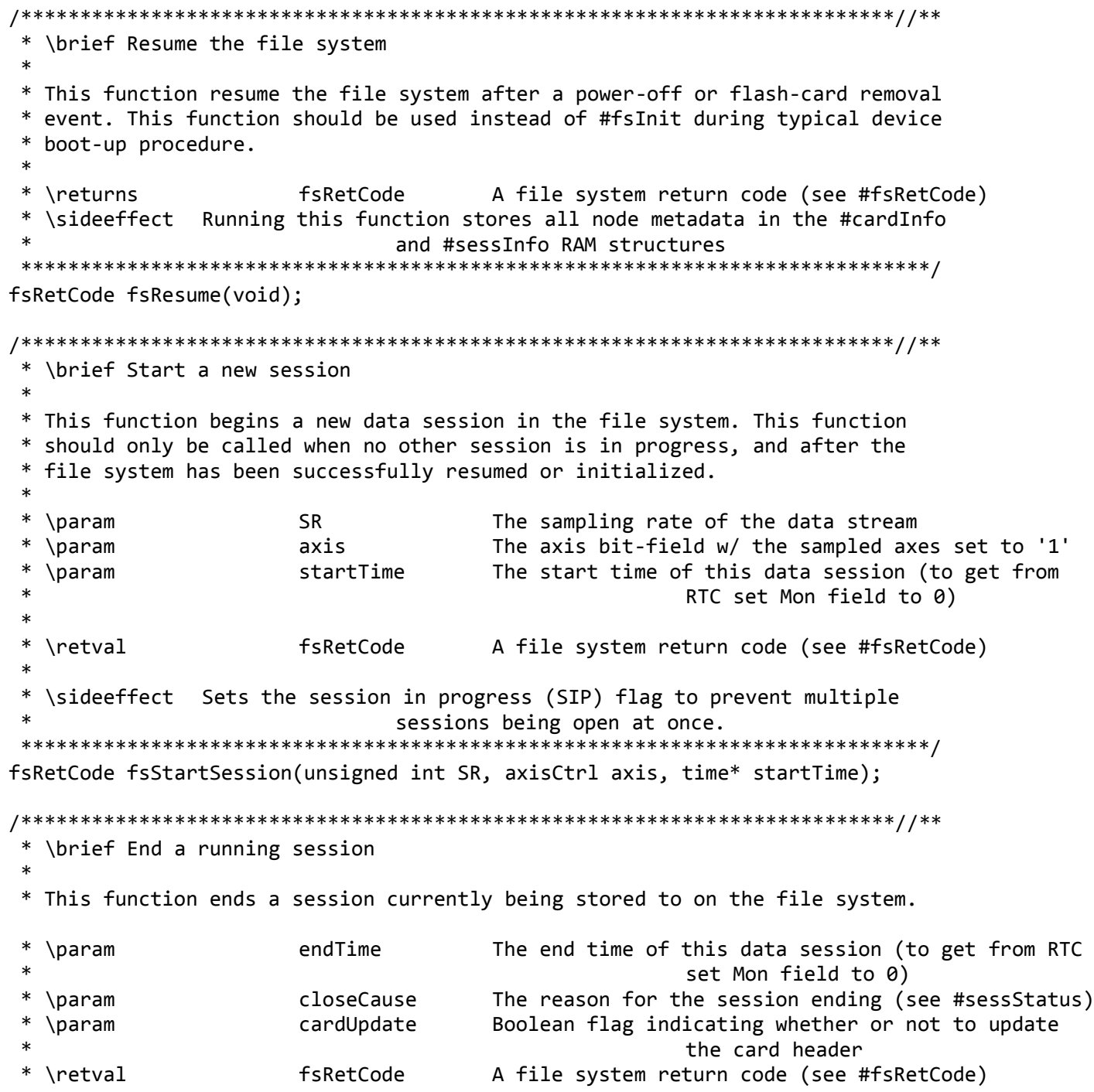


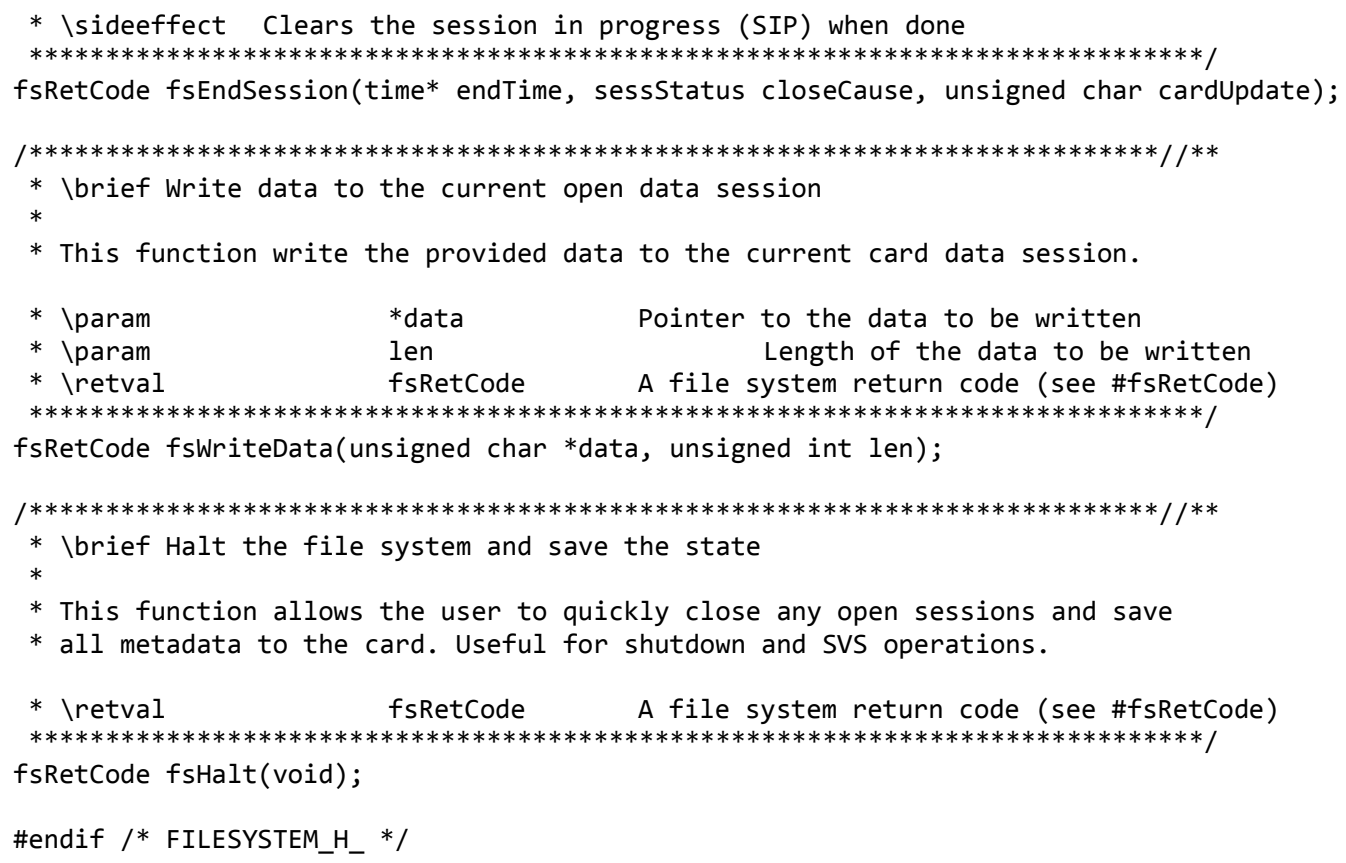

\section{Flash.c}

Basic system-level flash communications, built on top of MMC.c/h

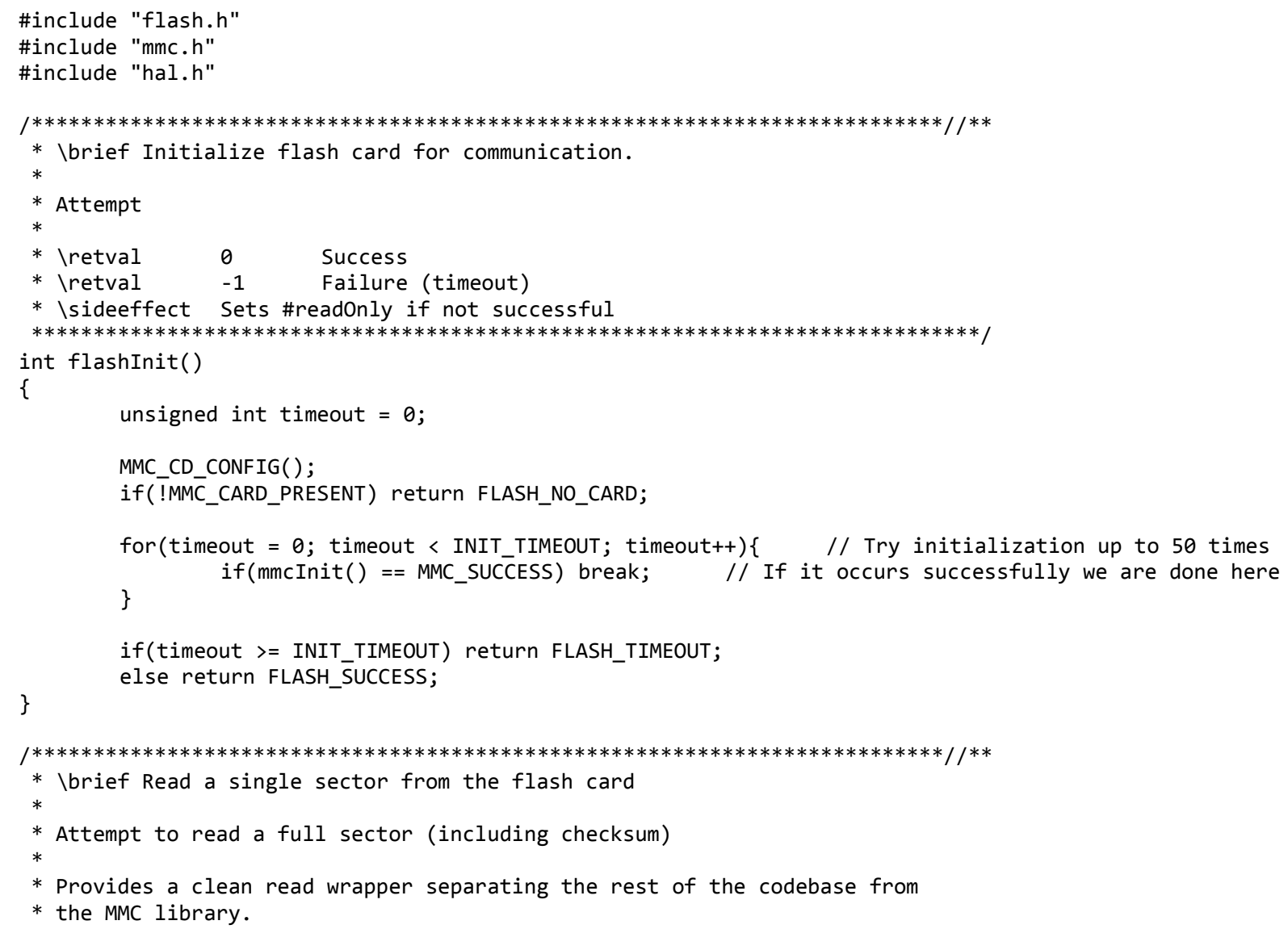




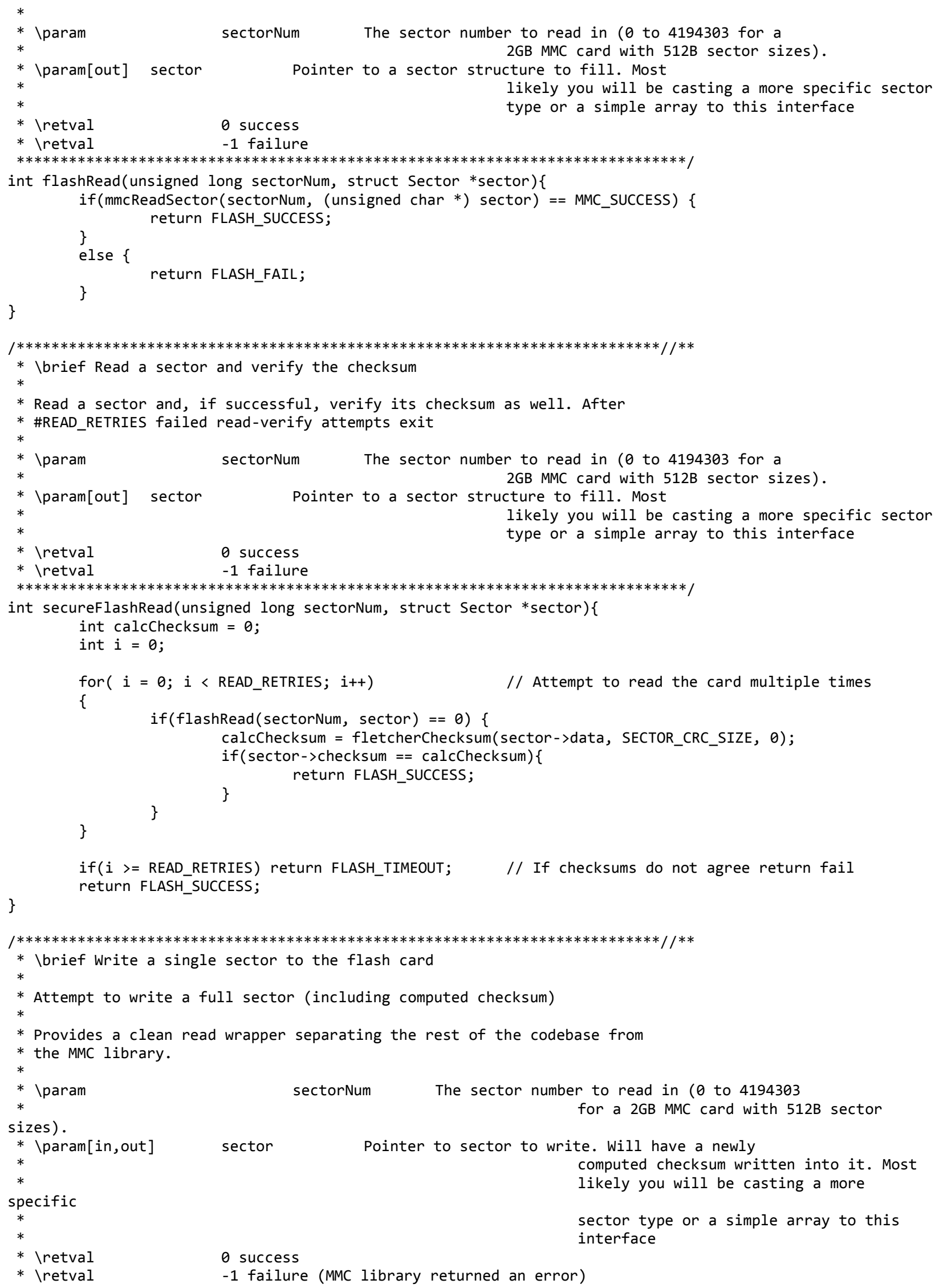




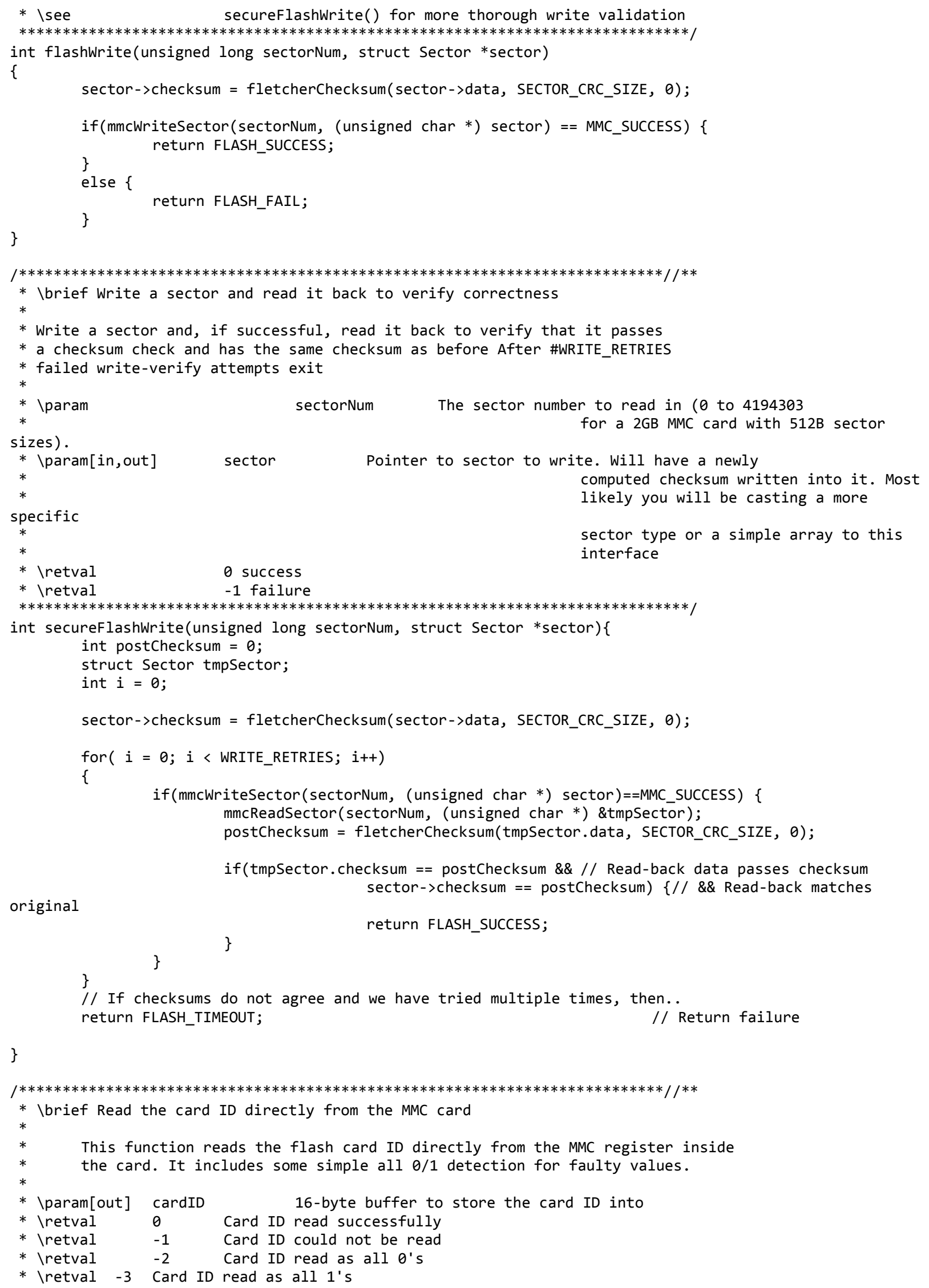




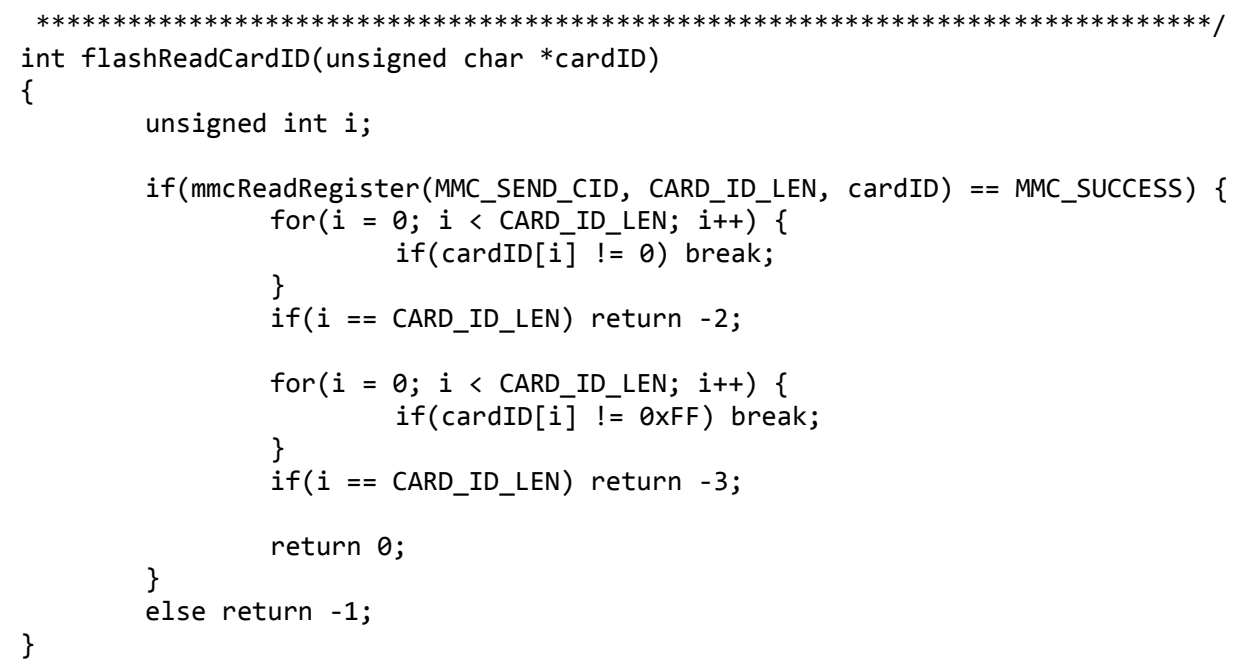

\section{Flash.h}

Affiliated flash header file

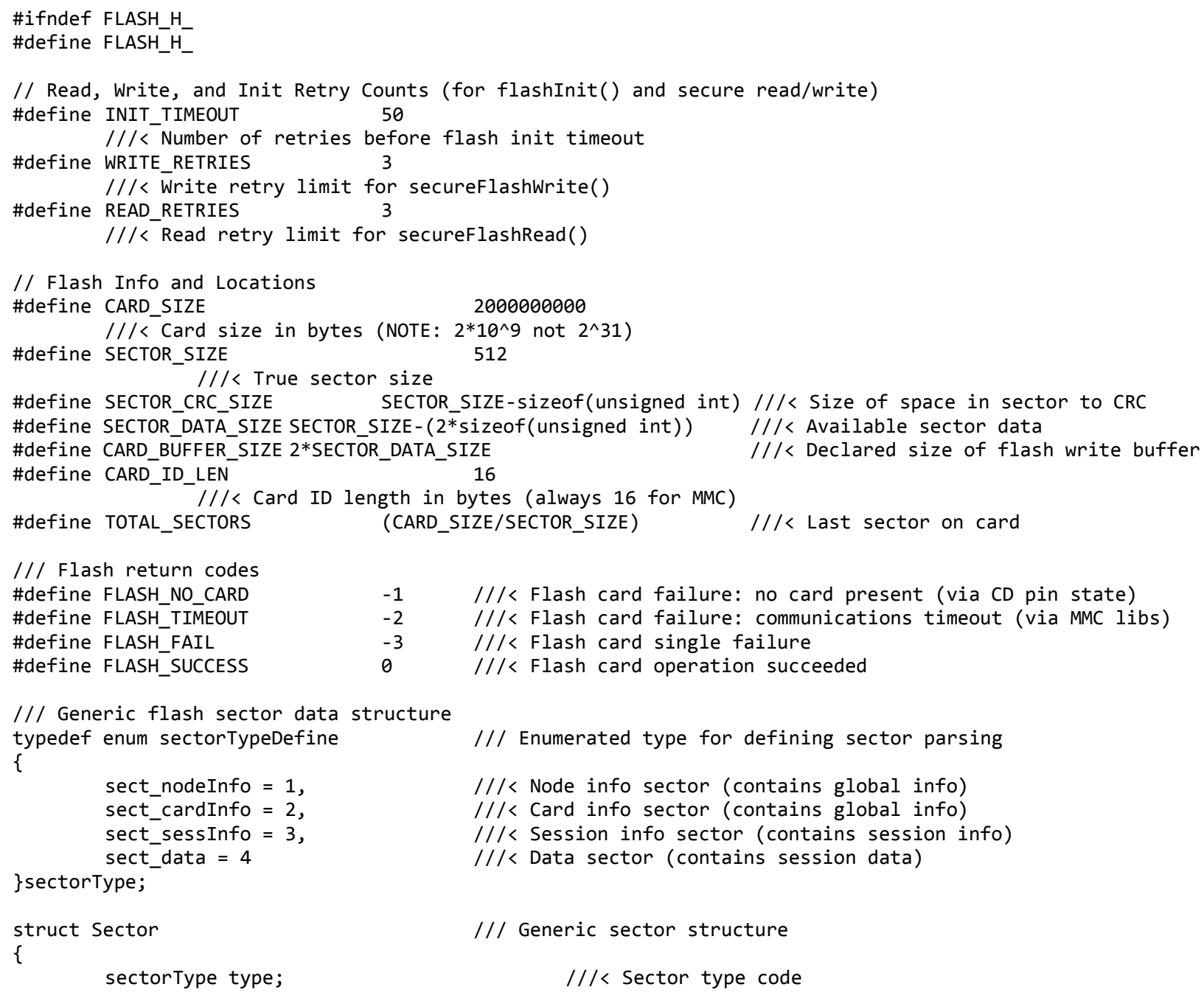

/// Generic sector structure

$$
/ / /<\text { Sector type code }
$$




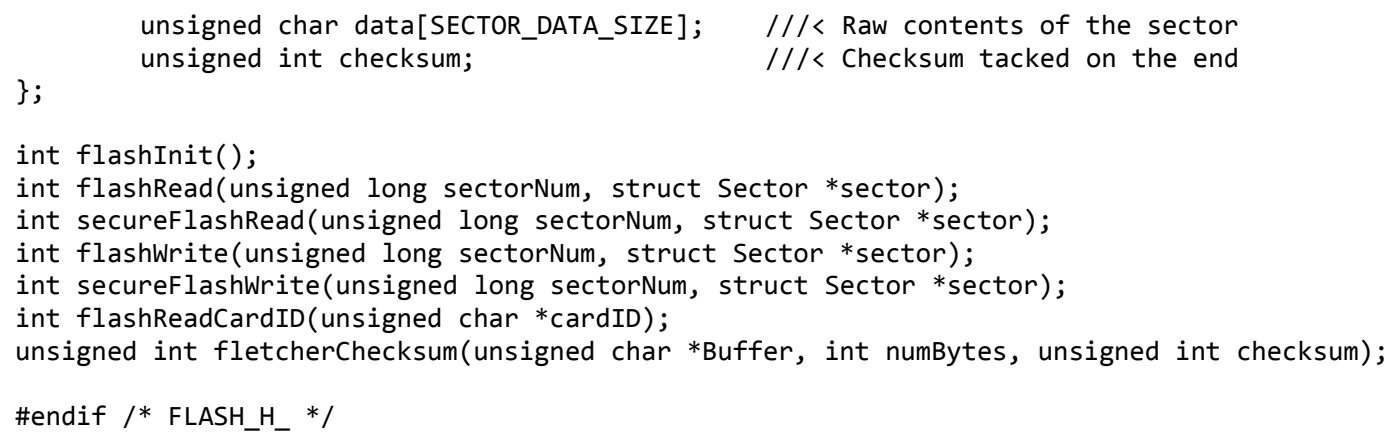

\section{Ftdi.c}

\section{Support driver for FT232 UART to USB translator written on top of comm.c}

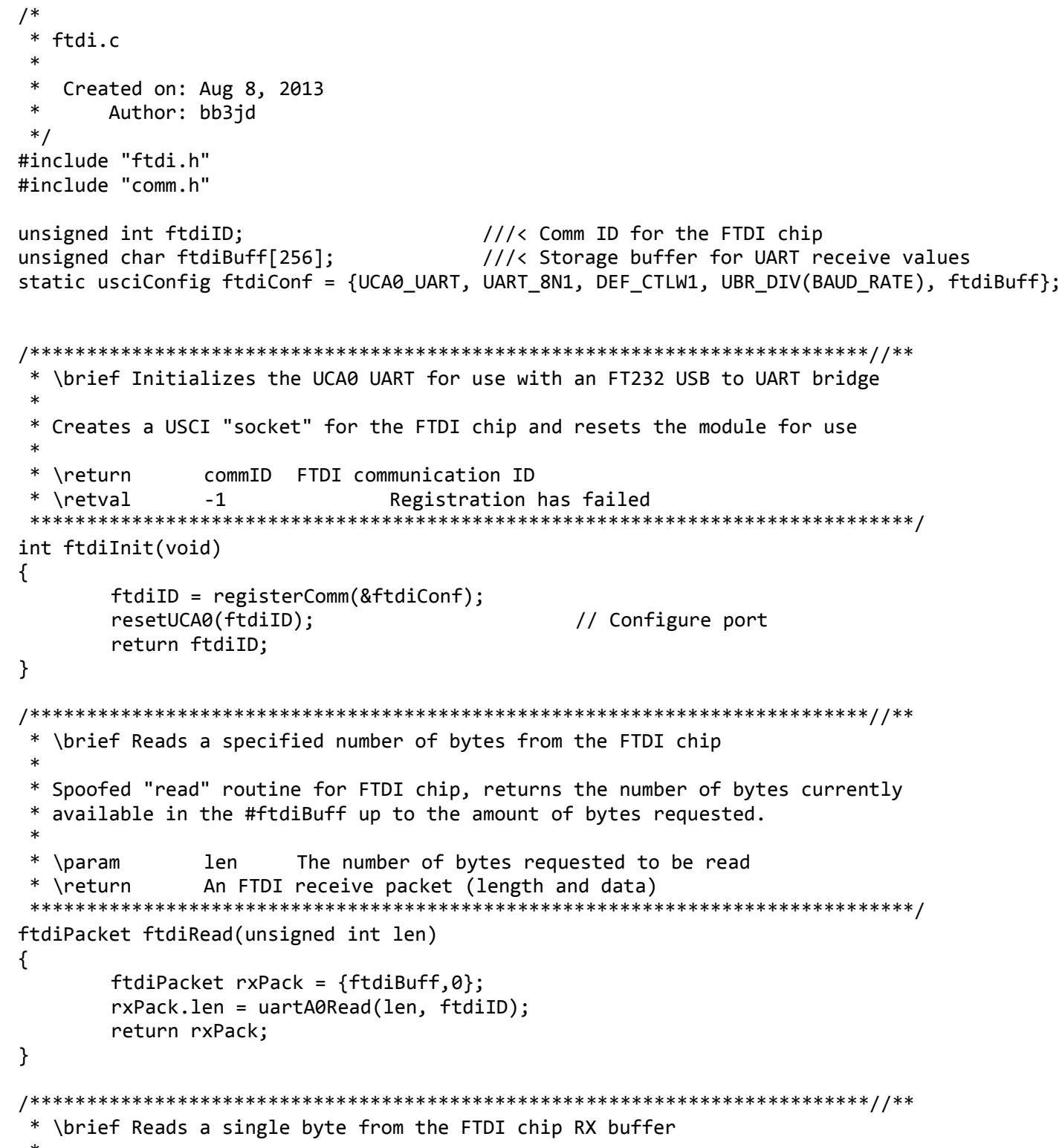




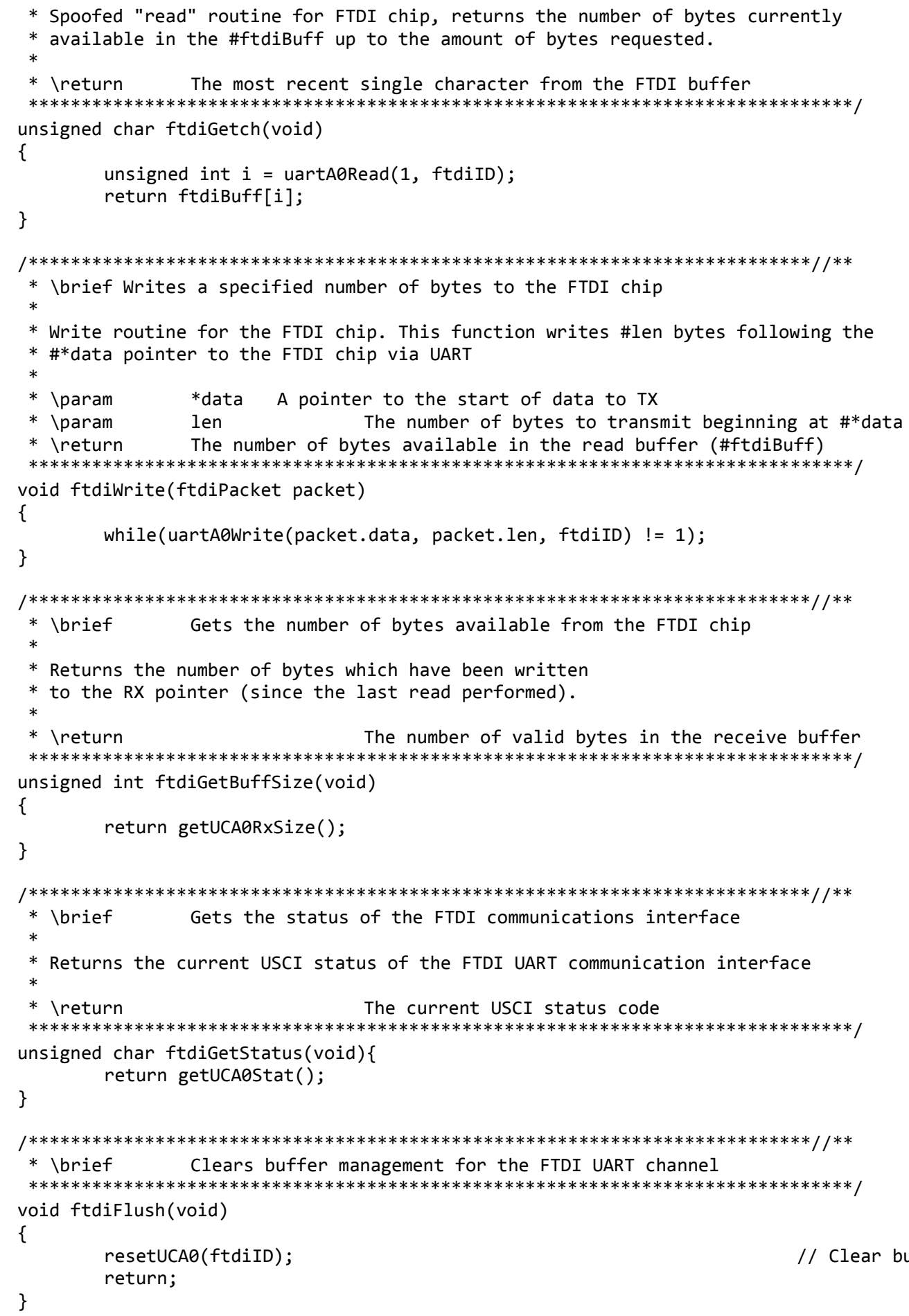

\section{Ftdi.h}

Affiliated FTDI header file

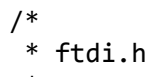




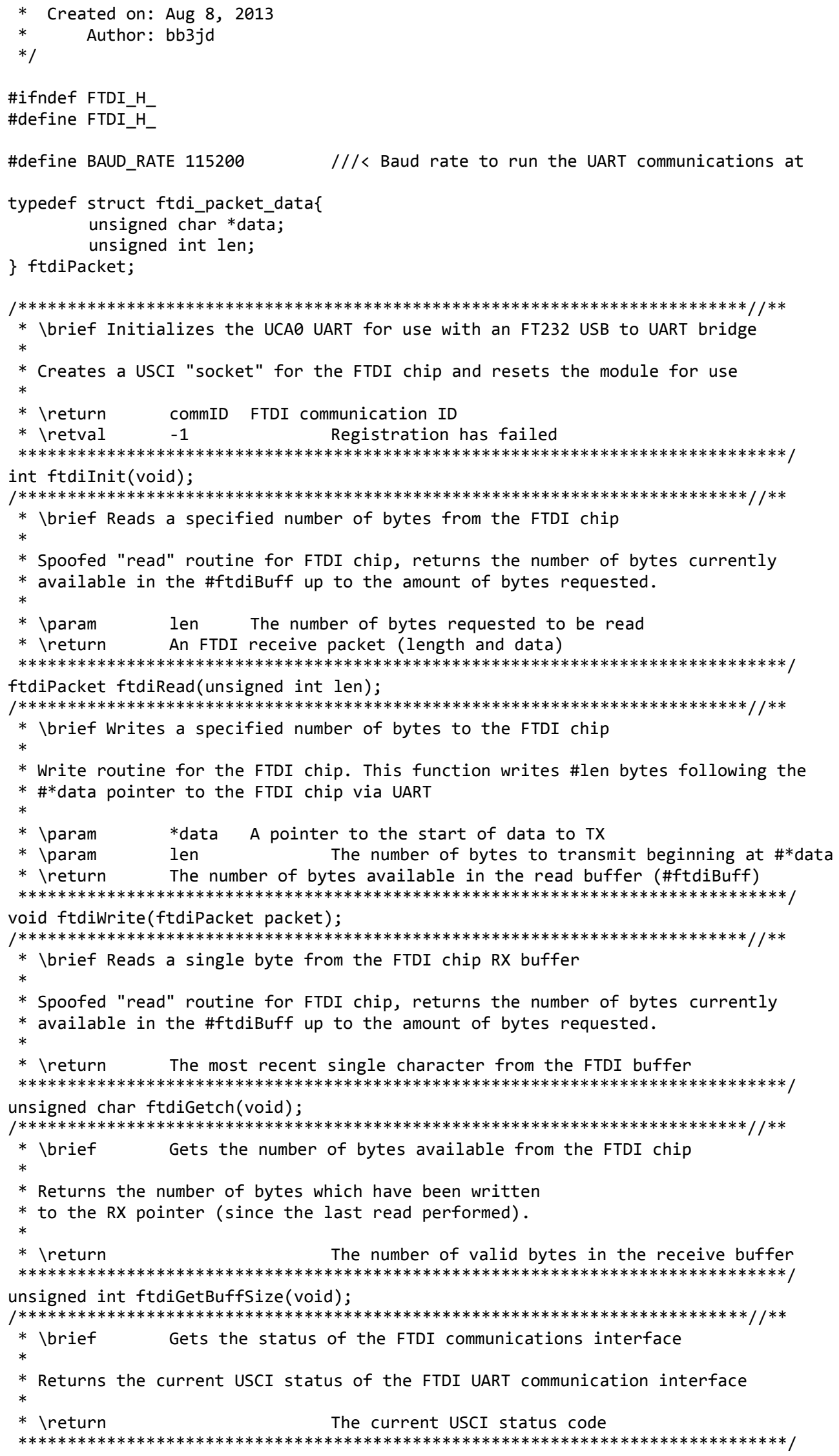


// LED 1 (Green) Functions \#define LED1_CONFIG()

\#define LED1_ON() ///< Set
\#define LED1_OFF( )
\#define LED1_TOGGLE( )
\#define LED_GREEN_CONFIG()
\#define LED_GREEN_ON()
\#define LED_GREEN_OFF()
\#define LED_GREEN_TOGGLE()
// LED 2 (Red) Functions
\#define LED2_CONFIG()

\#define

\#define LED2_OFF()

\#define LED2_TOGGLE()

\#define LED_RED_CONFIG()

\#define LED_RED_ON()

\#define LED_RED_OFF( )

\#define LED_RED_TOGGLE()

// General LED defines

\#define LED_CONFIG()

\#define LEDS_OFF()

\#define LEDS_ON()

// Switch 1 Functions

\#define SW1_CONFIG()

P1.2 to input w/ pull

\#define SW1_PRESSED

\#define SW1_RELEASED

// Switch 2 Functions

\#define SW2_CONFIG()

P1.3 to input w/ pull-up (switch

\#define SW2 PRESSED

\#define SW2_RELEASED

// General Switch Defines

\#define SW_CONFIG()

\#define SW_PRESSED

macro

\#define SW_RELEASED

// Ext. VCC Control Functions

\#define EXT VCC CONFIG()

$/ / \overline{<}$ Set $\mathrm{P} 1.4$ to output low (VCC2 off)

\#define EXT_VCC_ON( )

\#define EXT_VCC_OFF( )

// MAX1555 Charge Indi

\#define CHG_CONFIG()

\#define CHARGING

// FT232 USB Power Indicator Functions

1DS |= BIT0; P10UT |= BIT0; P1DIR |= BIT0; P1SEL \&= (BIT0)

P1.0 to output high (LED off)

LED1 = $0 \quad / / /<$ LED 1 on macro

LED1 = $1 \quad / / /<$ LED 1 off macro

LED1 $\wedge=1 \quad / / /<$ LED 1 toggle macro

LED1_CONFIG() $\quad / / /<$ Rename macro for green LED configuration

LED1_ON() $\quad / / /<$ Rename macro for green LED (LED1) on

LED1_OFF() $\quad / / /<$ Rename macro for green LED (LED1) off

LED1_TOGGLE() $\quad / / /<$ Rename macro for green LED (LED1) toggle

P1DS $\mid=$ BIT1; P10UT $\mid=$ BIT1; P1DIR $\mid=$ BIT1; P1SEL \&= $($ BIT1)

high (LED off)

LED2 $=0 \quad / / /<$ LED2 on macro

LED2 = $1 \quad / / /<$ LED2 off macro

LED2 $\wedge=1 \quad / / /<$ LED2 toggle macro

LED2_CONFIG() $\quad / / /<$ Rename macro for red LED configuration

LED2_ON() $\quad / / /<$ Rename macro for red LED (LED2) on

LED2_OFF() $\quad / / /<$ Rename macro for red LED (LED2) off

LED2_TOGGLE() $/ / /<$ Rename macro for red LED (LED2) toggle

LED1_CONFIG(); LED2_CONFIG() $\quad / / /<$ Group LED configuration

LED1_OFF ( ); LED2_OFF( ) $/ / /<$ Group LED off

LED1_ON(); LED2_ON( ) ///<Group LED on

P10UT |=BIT2; P1REN |=BIT2; P1DIR \&= (BIT2); P1SEL \&= (BIT2) $/ / /<$ Set pulls down)

SW1 $=0 \quad / / /<$ SW1 depressed (closed) macro

SW1 == $1 \quad / / /<$ SW1 released (open) macro

P10UT |= BIT3; P1REN |= BIT3; P1DIR \&= (BIT3); P1SEL \& $=\sim($ BIT3 $) / / /<$ Set pulls down)

SW2 == $0 \quad / / /<$ SW2 depressed (closed) macro

SW2 == $1 \quad / / /<$ SW2 released (open) macro

SW1_CONFIG(); SW2_CONFIG() $\quad / / /<$ Group switch config

SW1_PRESSED | SW2_PRESSED $\quad / / /<$ Group switch depressed (closed)

SW1_RELEASED \& SW2_RELEASED $\quad / / /<$ Group switch released (open) macro

P10UT \&= (BIT4); P1DIR |= BIT4; P1SEL \& $=\sim($ BIT4)

P10UT \&= (0x10) $\quad / / /<$ External VCC off macro

P1REN \&= (BIT5); P1DIR $\&=\sim($ BIT5 $)$; P1SEL $\&=\sim($ BIT5 $)$

\#define USB_VCC_CONFIG() P10UT \&= (BIT6); P1DIR \&= (BIT6); P1SEL \&= $($ BIT6) //; P1REN |= BIT6 ///< Set

P1.6 to input ( $\bar{w} / 0$ pull-down)

\#define USB_VCC_ON

\#define USB_VCC_OFF

// MPU Pin Indicator Functions

\#define MPU_INT_CONFIG() P1REN $\&=\sim($ BIT7); P1SEL $\&=\sim($ BIT7); P1DIR $\&=\sim($ BIT7) $/ / /<$ Set P1.7 to input (no pull-up/down)

$\begin{aligned} &\text { \#define MPU_FSYNC_CONFIG( }) \\ & / / /<\text { Set } \mathrm{P} 2.7 \text { to output } 1\end{aligned}$

\#define MPU INT HIGH MPU INT $==1$

\#define MPU_INT_LOW $\quad$ MPU_INT $==0$

\#define MPU_FSYNTC_ON( )

\#define MPU_FSYNC_OFF( )

\#define MPU_IO_CONFIG()

// MMC Pin Indicator functions

\#define MMC_IO_CONFIG()

\#define MMC_CS_CONFIG()

$1 / /<\mathrm{Set}$

\#define MMC_CD_CONFIG()

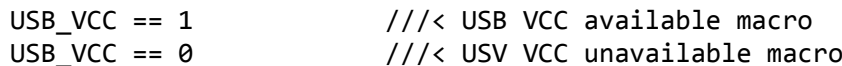

MPU_FSYNC $=0$

$/ / /<$ Charging macro MPU_INT_CONFIG() //; MPU_FSYNC_CONFIG() /// MPU IO initialization macro

MMC_CS_CONFIG( ); MMC_CD_CONFIG( )

P5DIR $T=$ BIT2; P5SEL $\&=\sim(B I T 2) ;$ P5OUT $\mid=$ BIT2

output high (not selected)

P5.3 to input (w/ pull-up)
P5OUT |= BIT3; P5REN |= BIT3; P5DIR \&= (BIT3); P5SEL \&= $($ BIT3) $/ / /<$ Set 


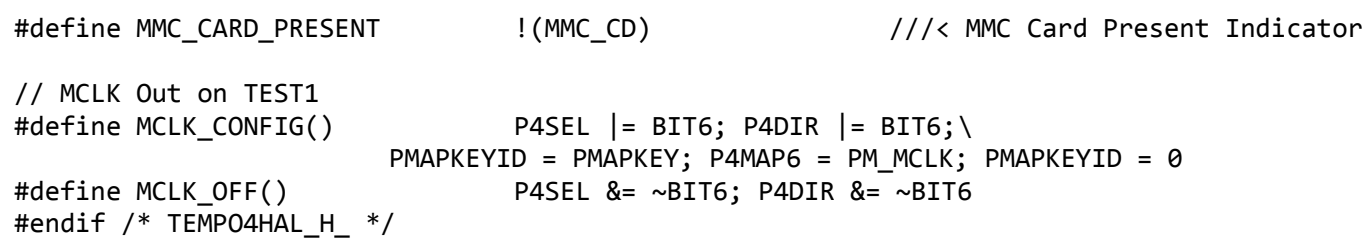

\section{Infoflash.c}

System information flash management, ported from TEMPO 3.2 for use on the MSP430F5342

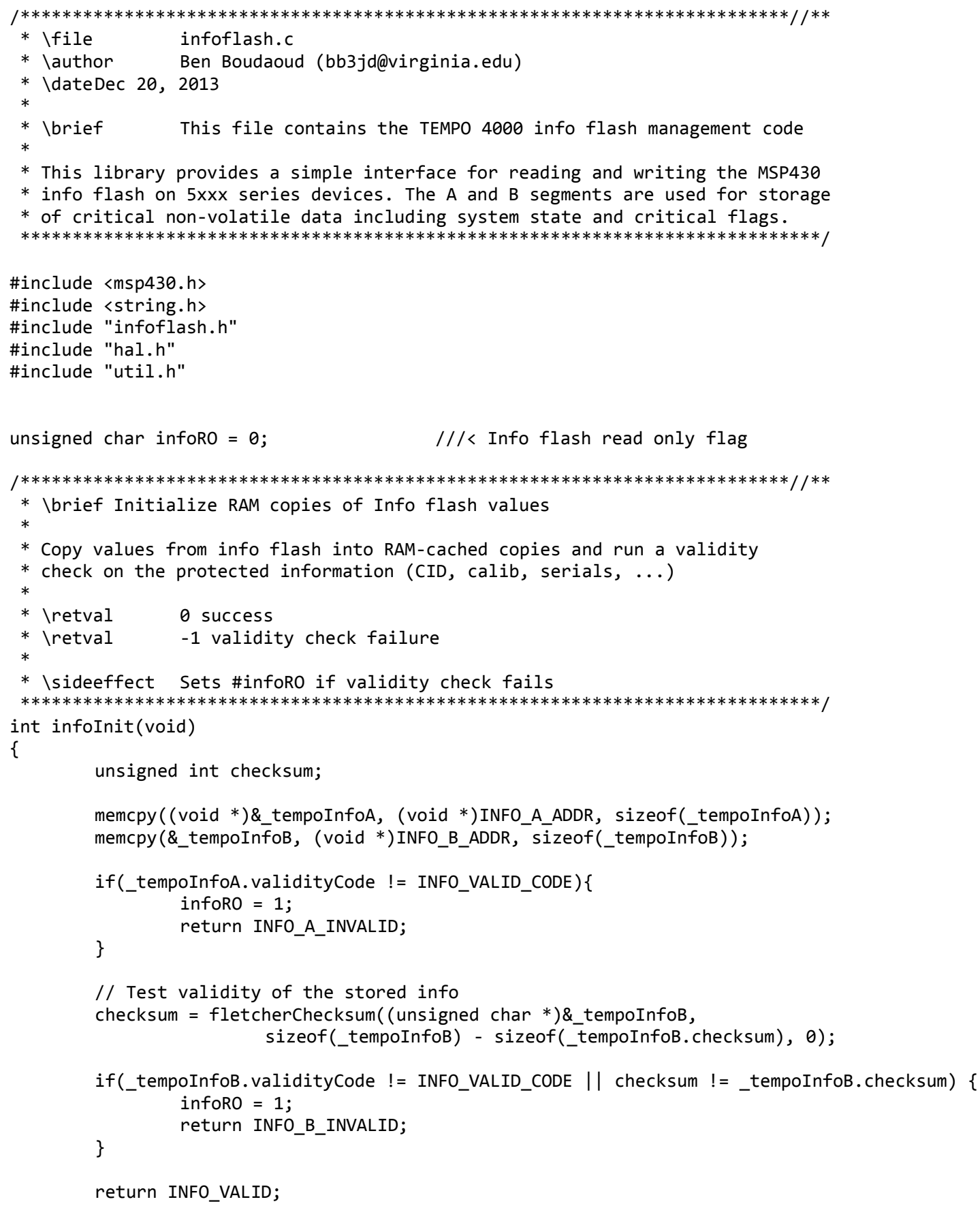




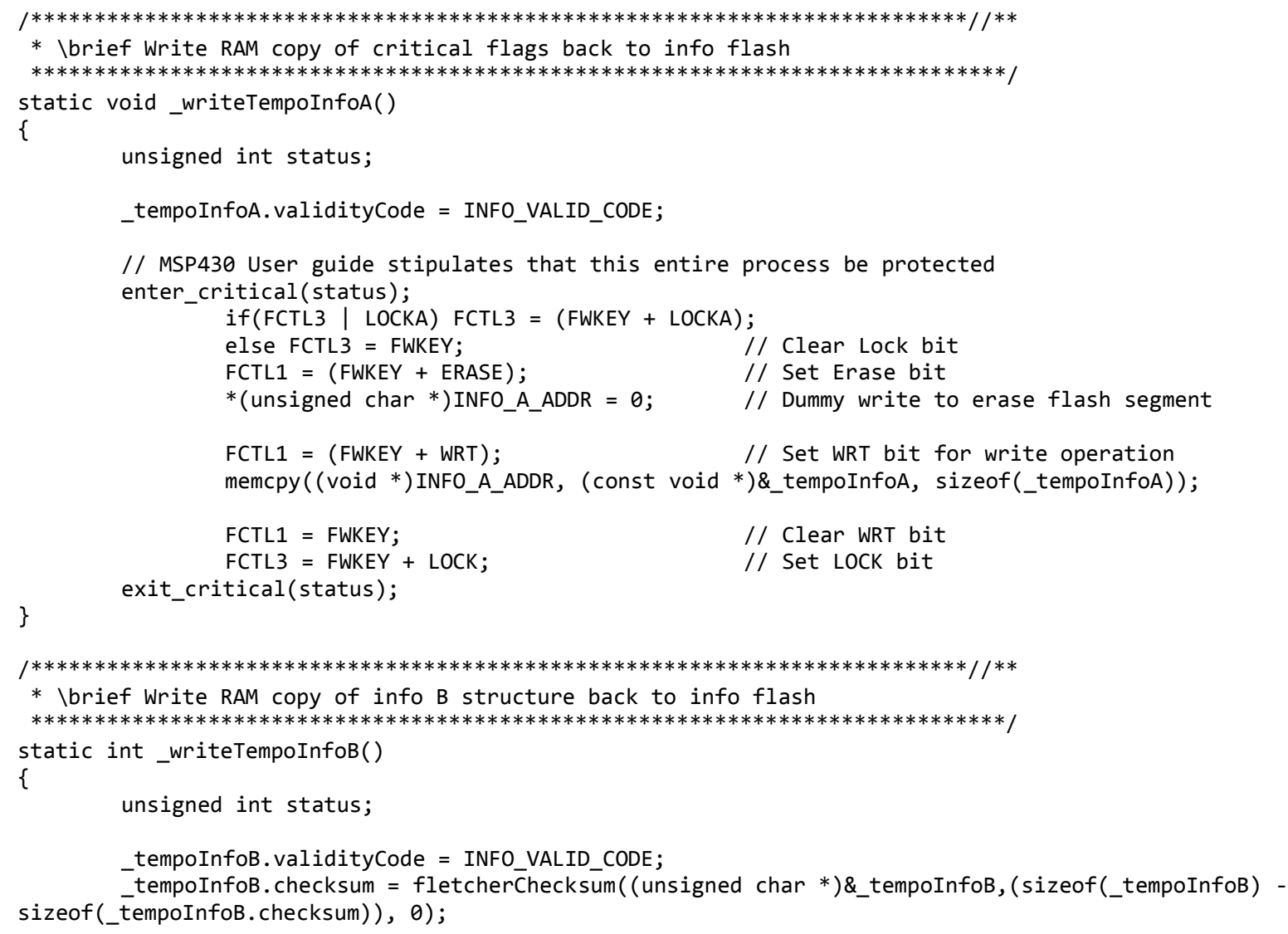




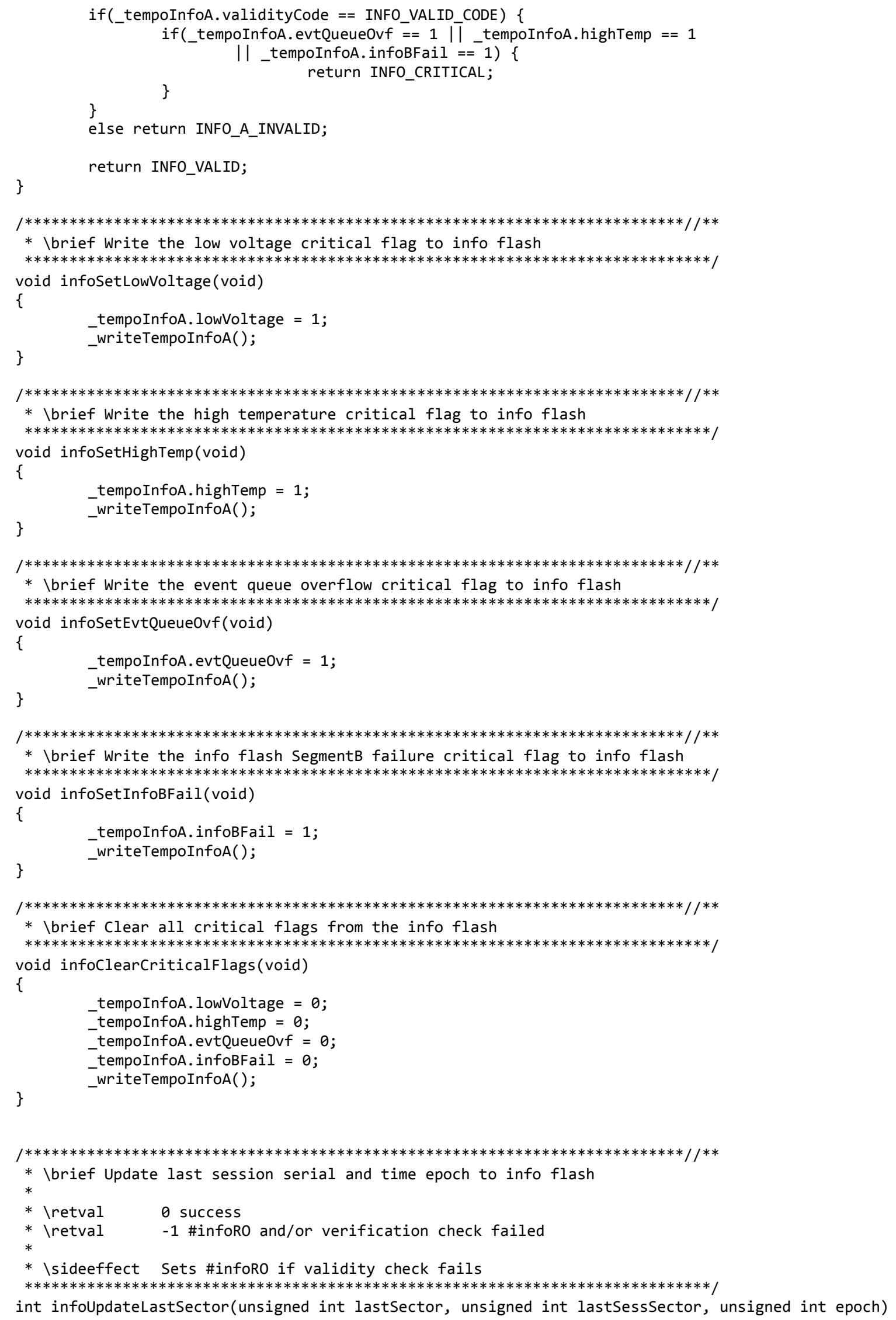




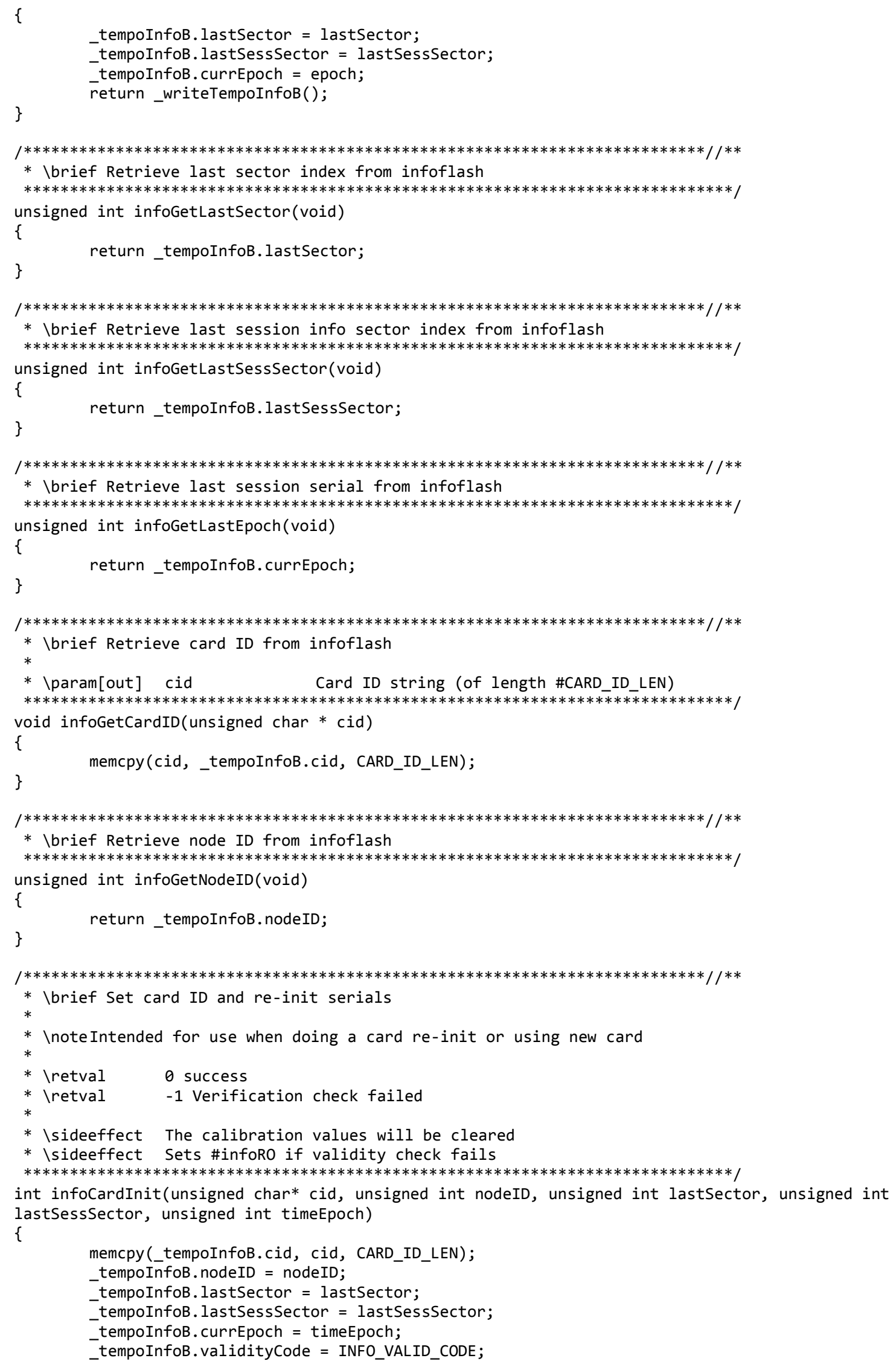


return _writeTempoInfoB();

\section{Infoflash.h}

Affiliated information flash header file, ported from TEMPO 3.2 header file

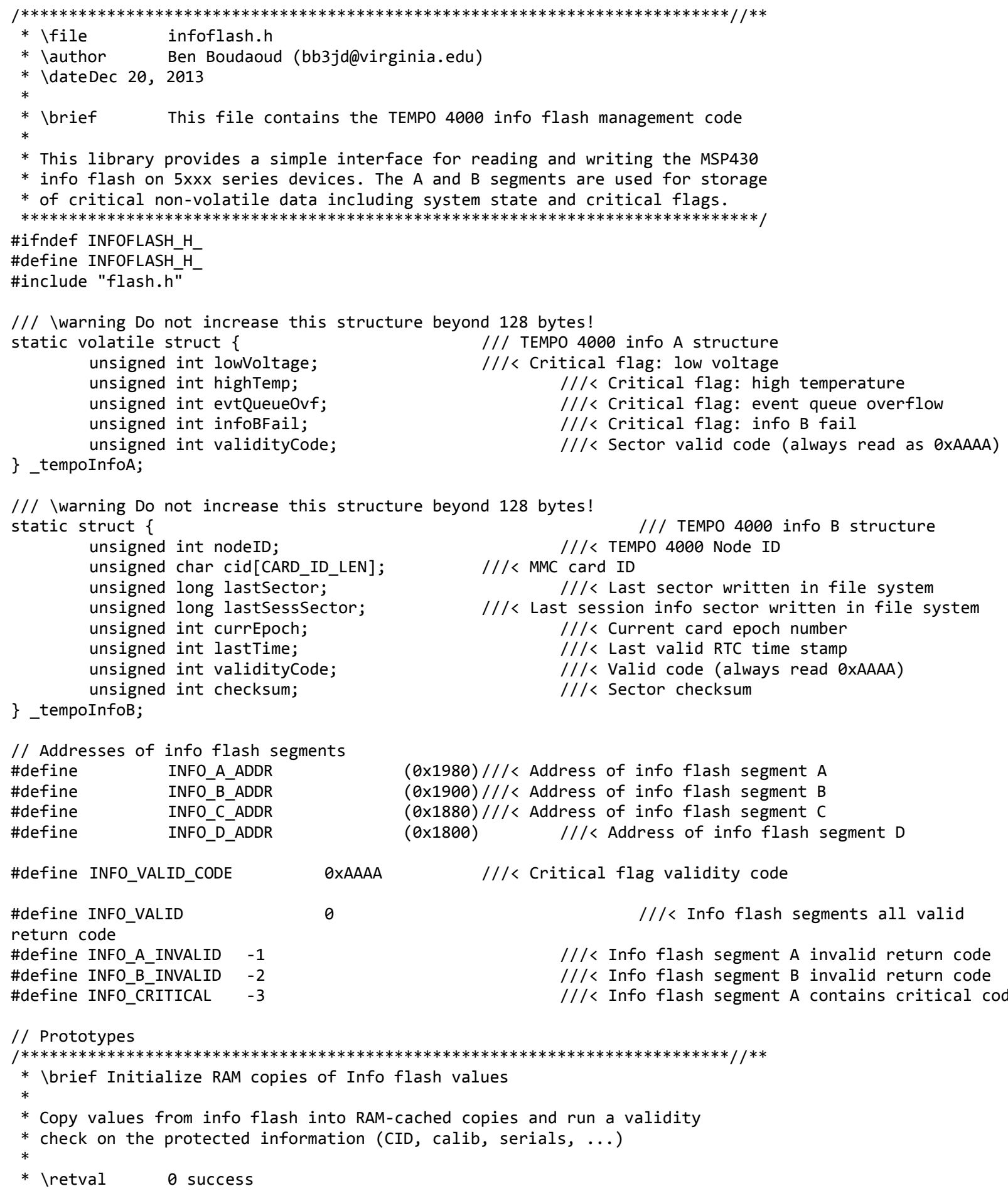

* \retval $\quad 0$ success 


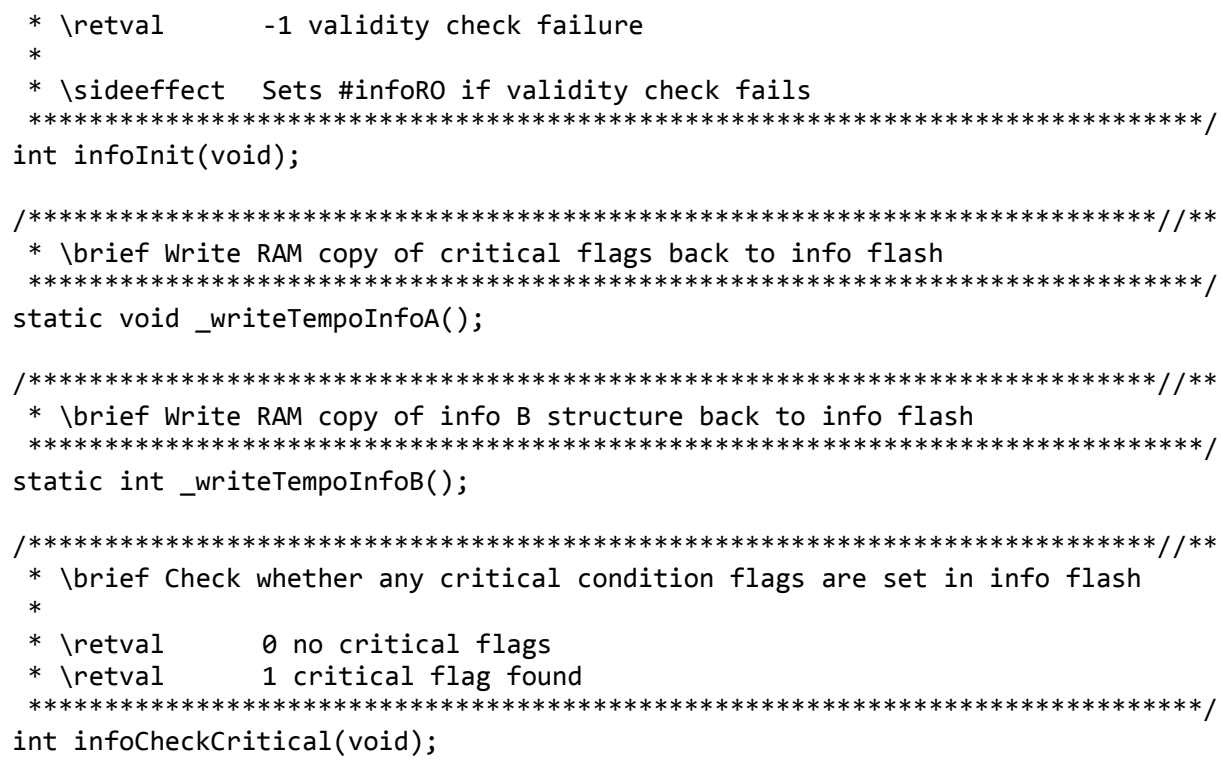




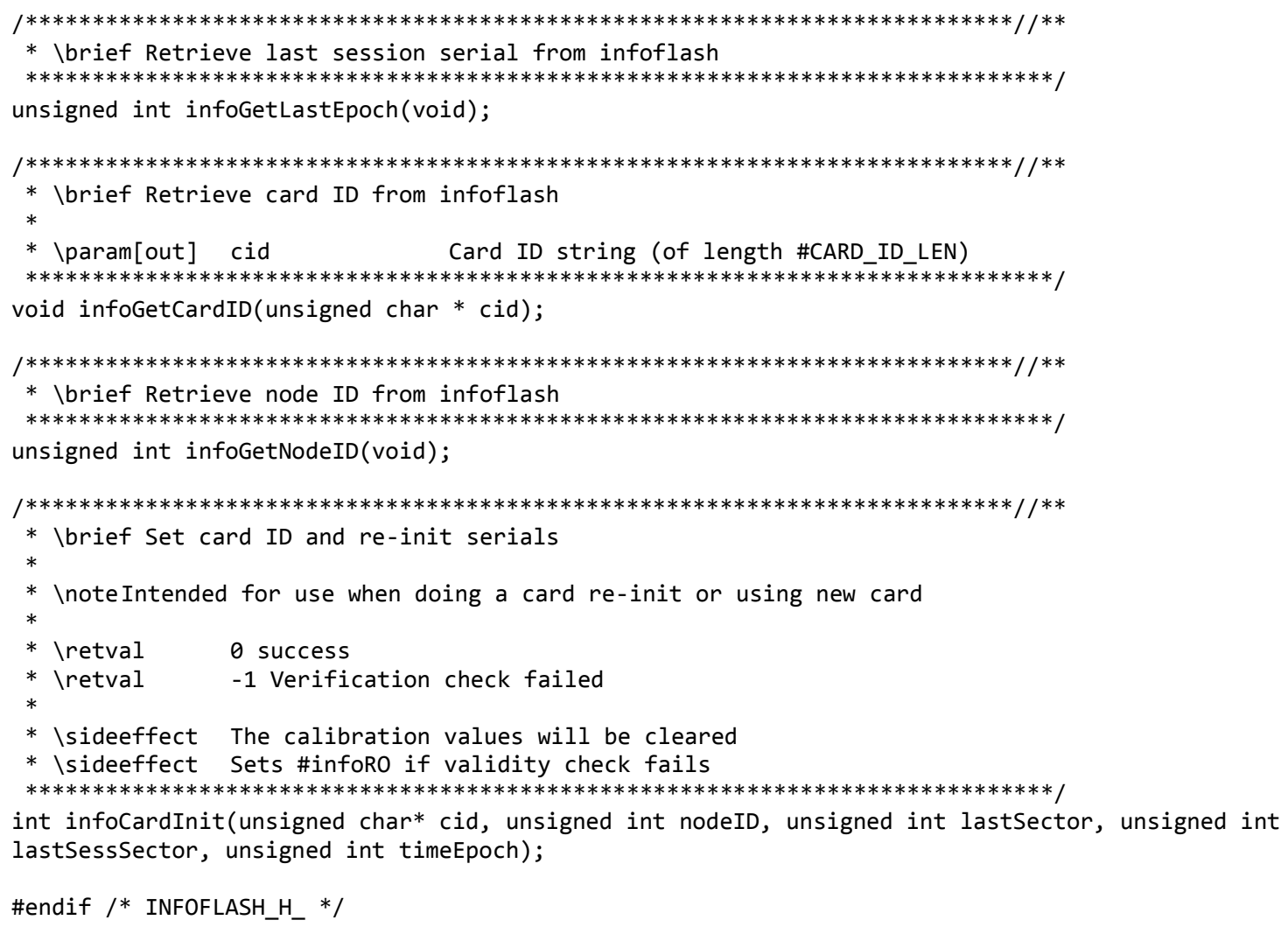

\section{Interrupts.c}

System interrupt masking and registration library, can be expanded to include any amount of user/developer registered interrupts

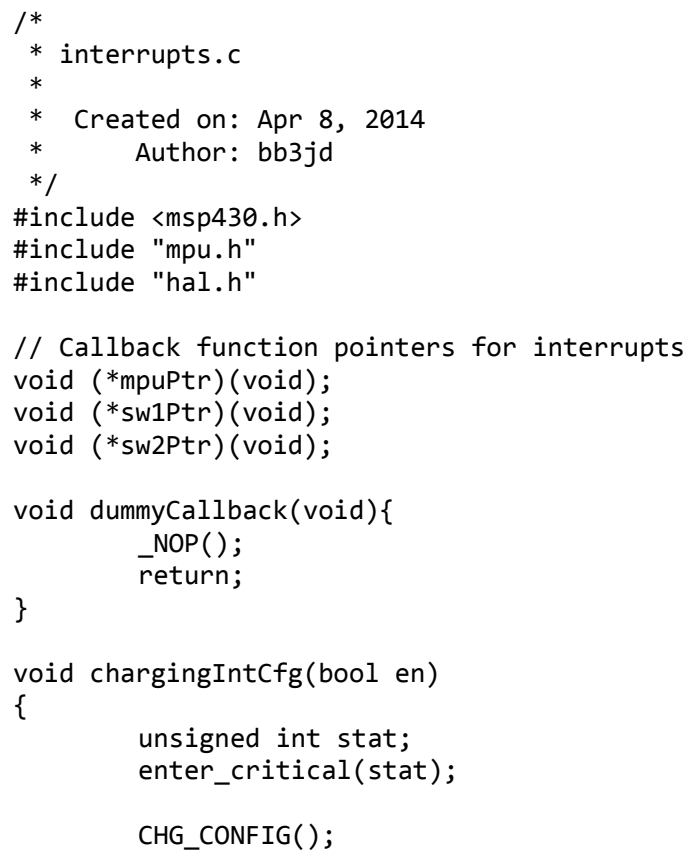




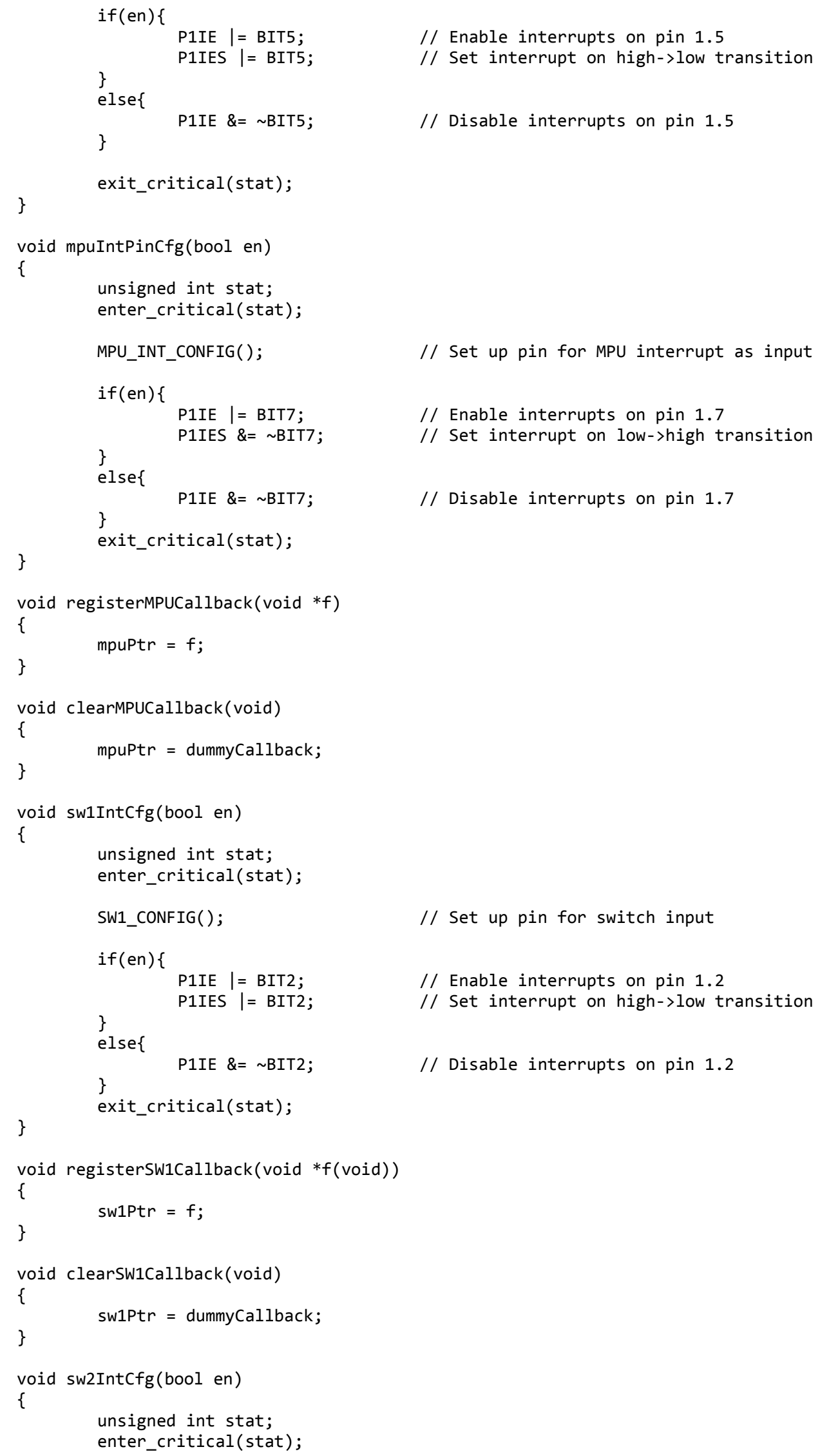




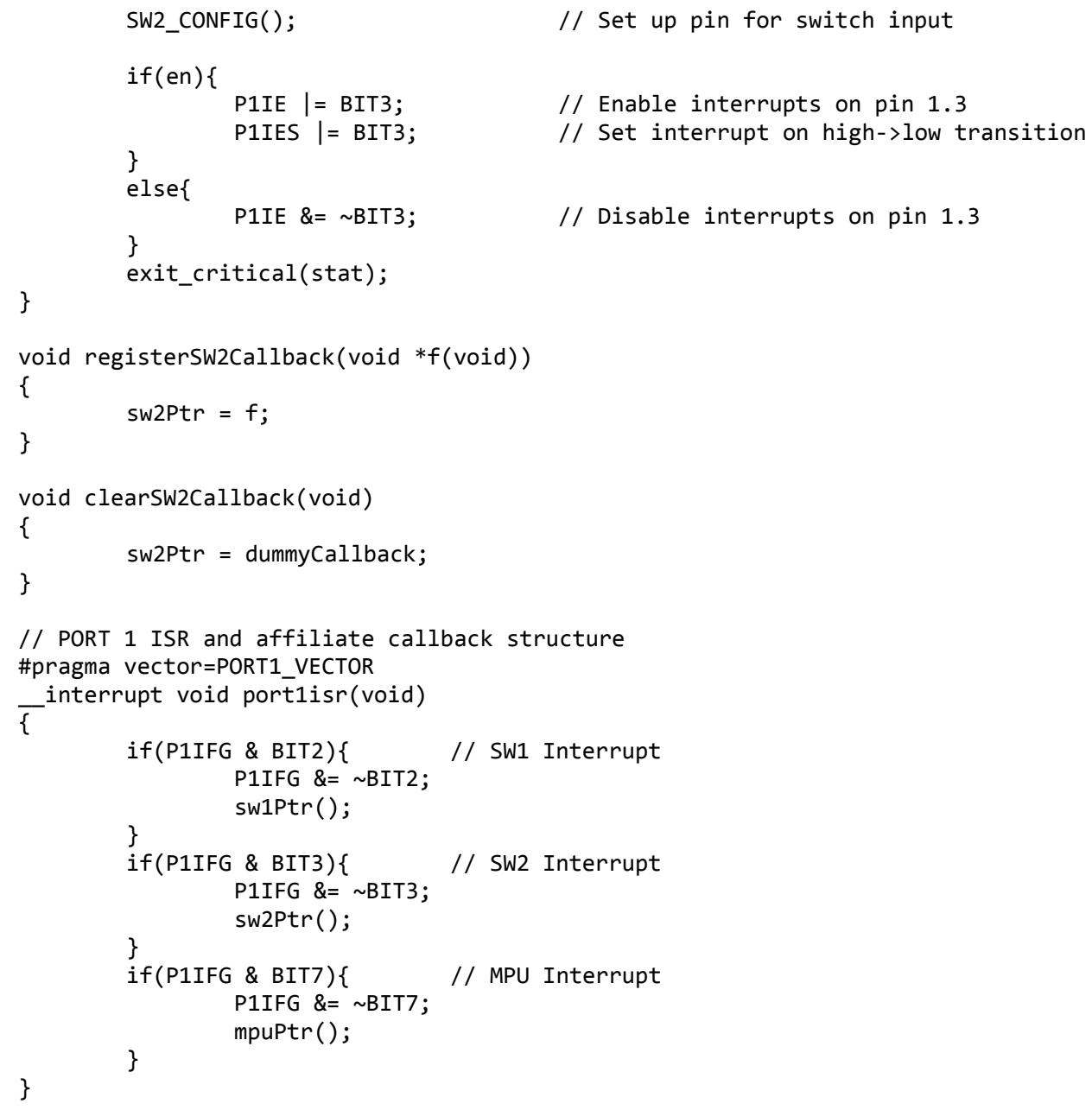

\section{MMC.c}

System MMC communication driver, ported from TEMPO 3.2 for use with communications library

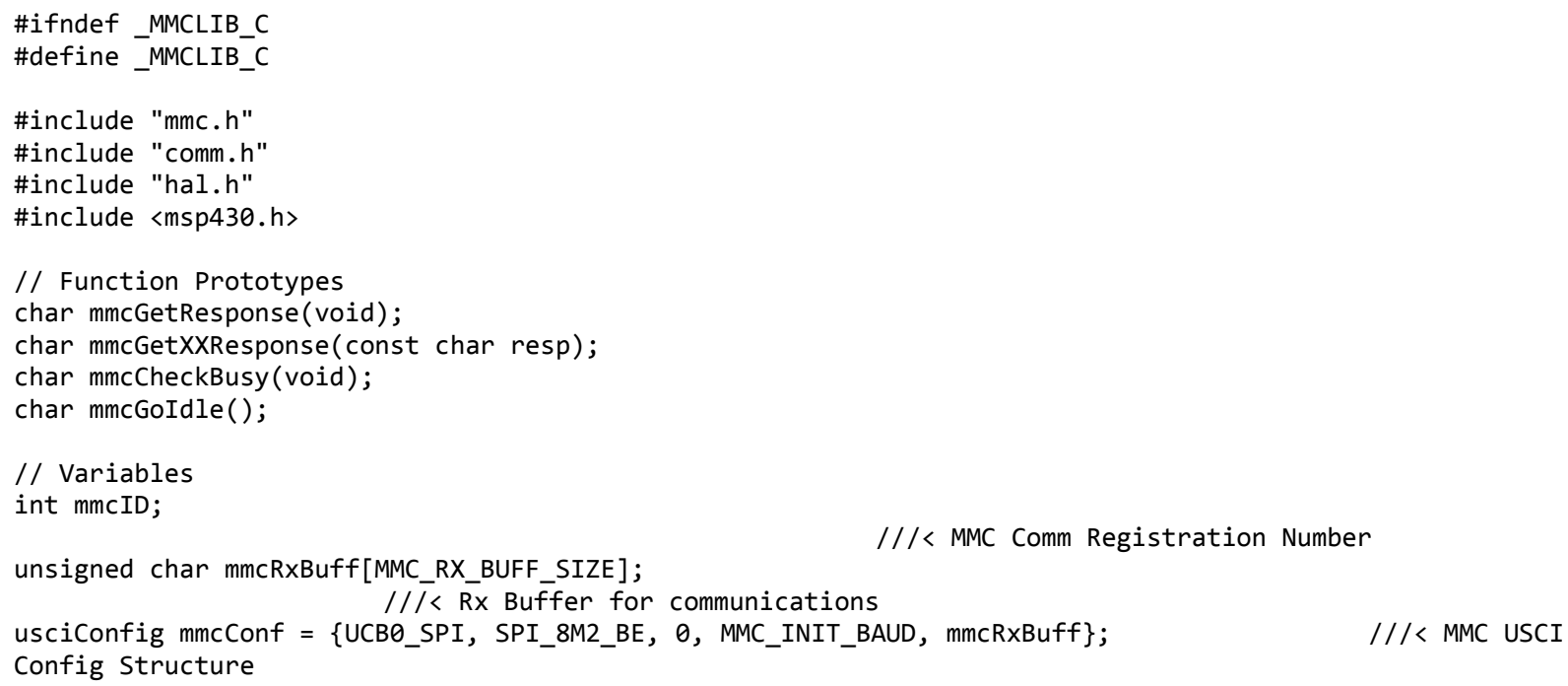




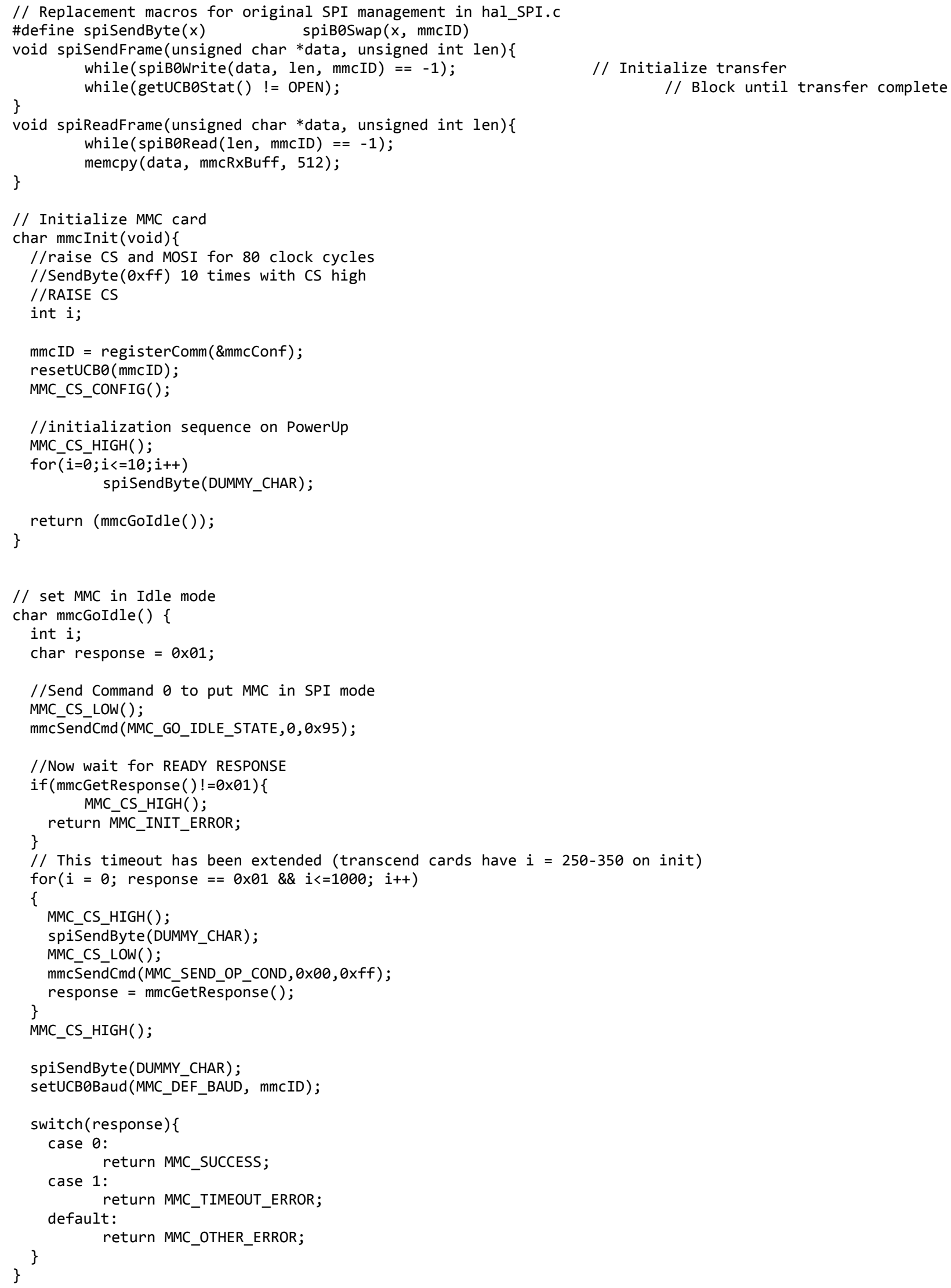




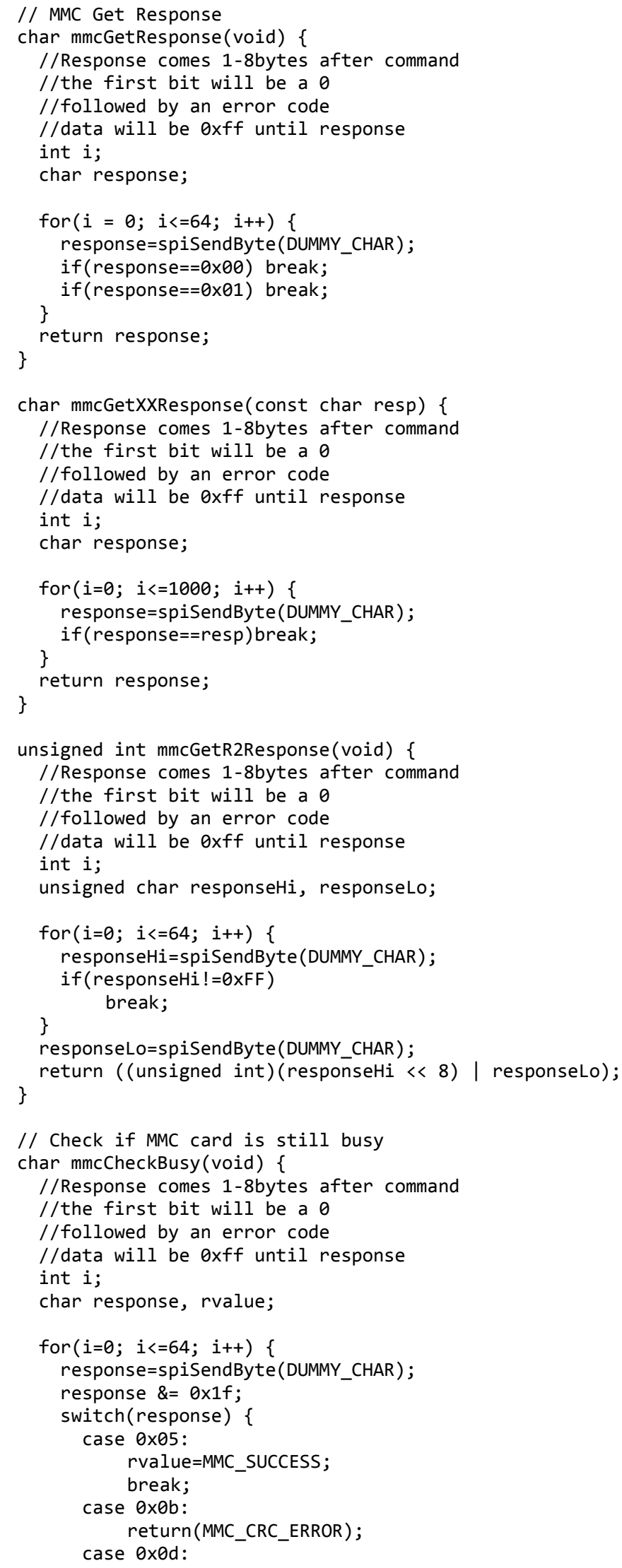




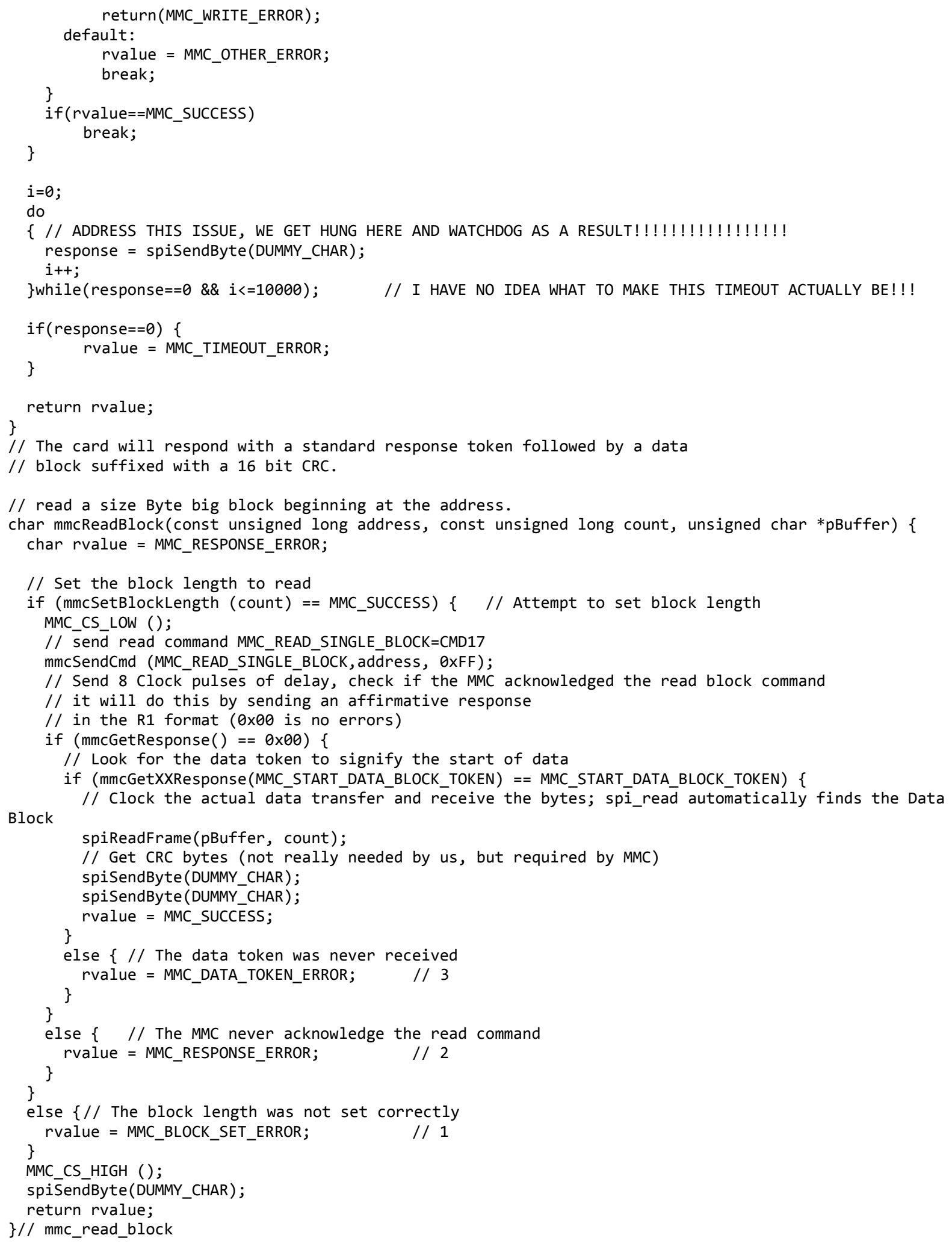




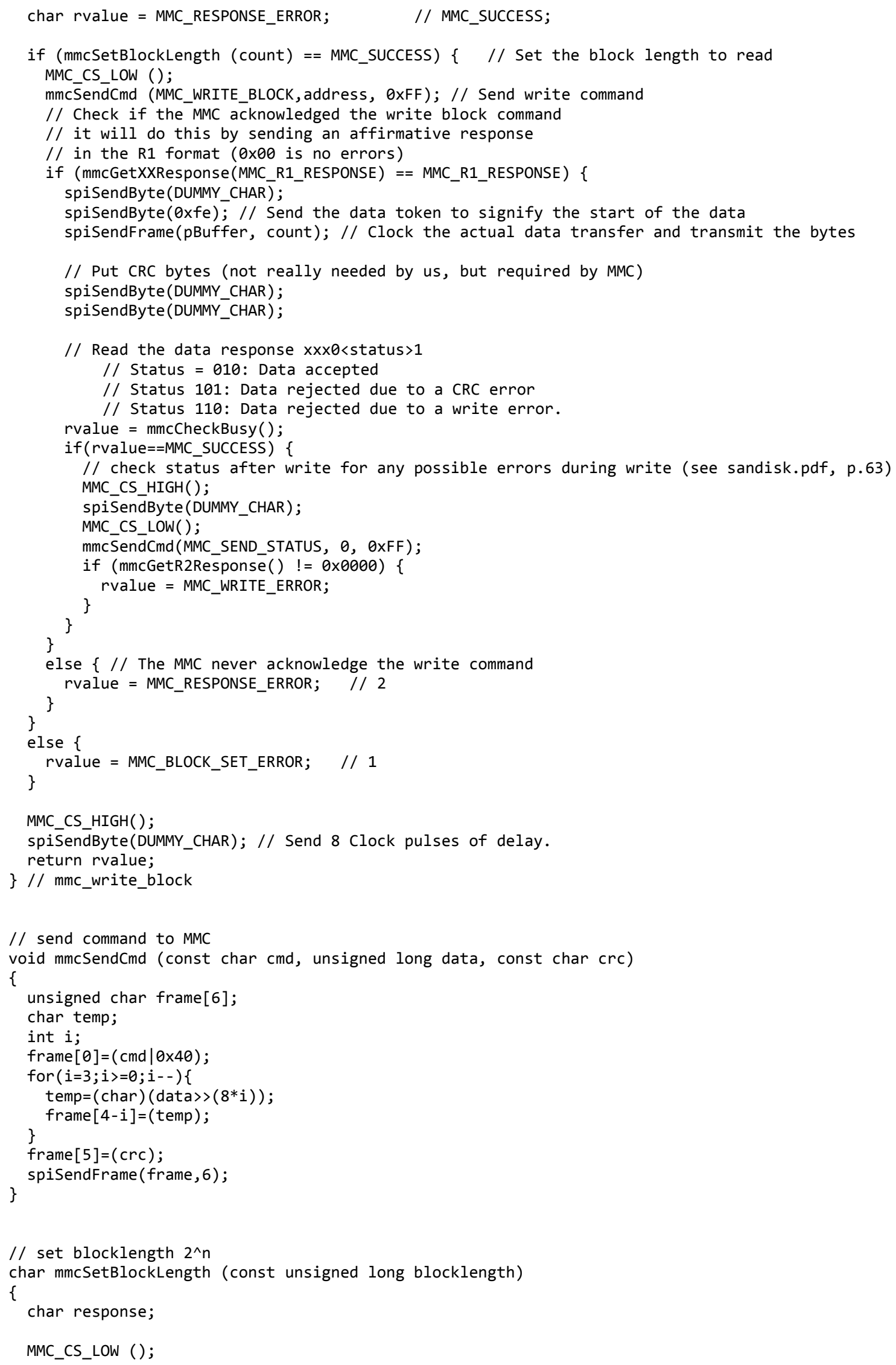




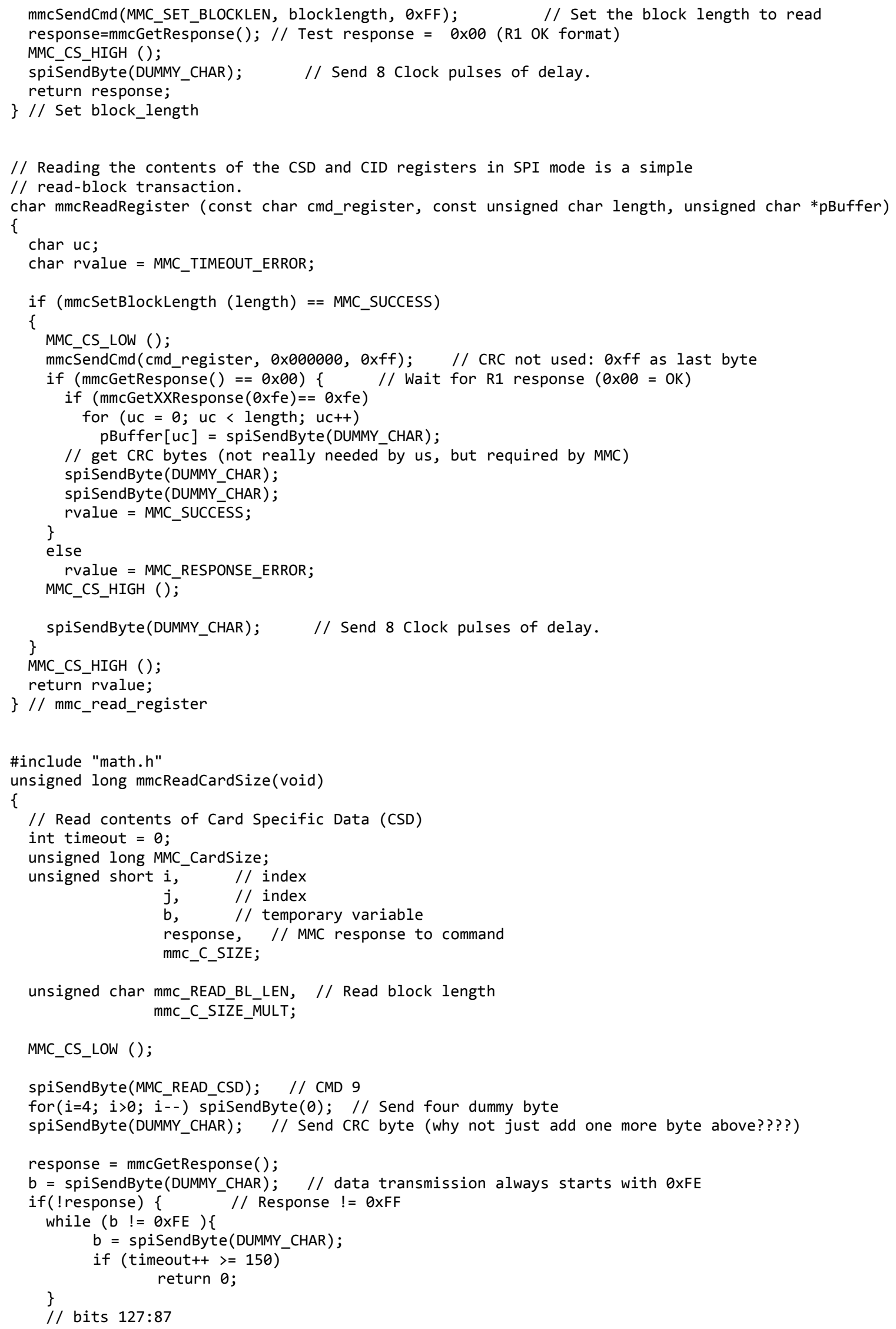




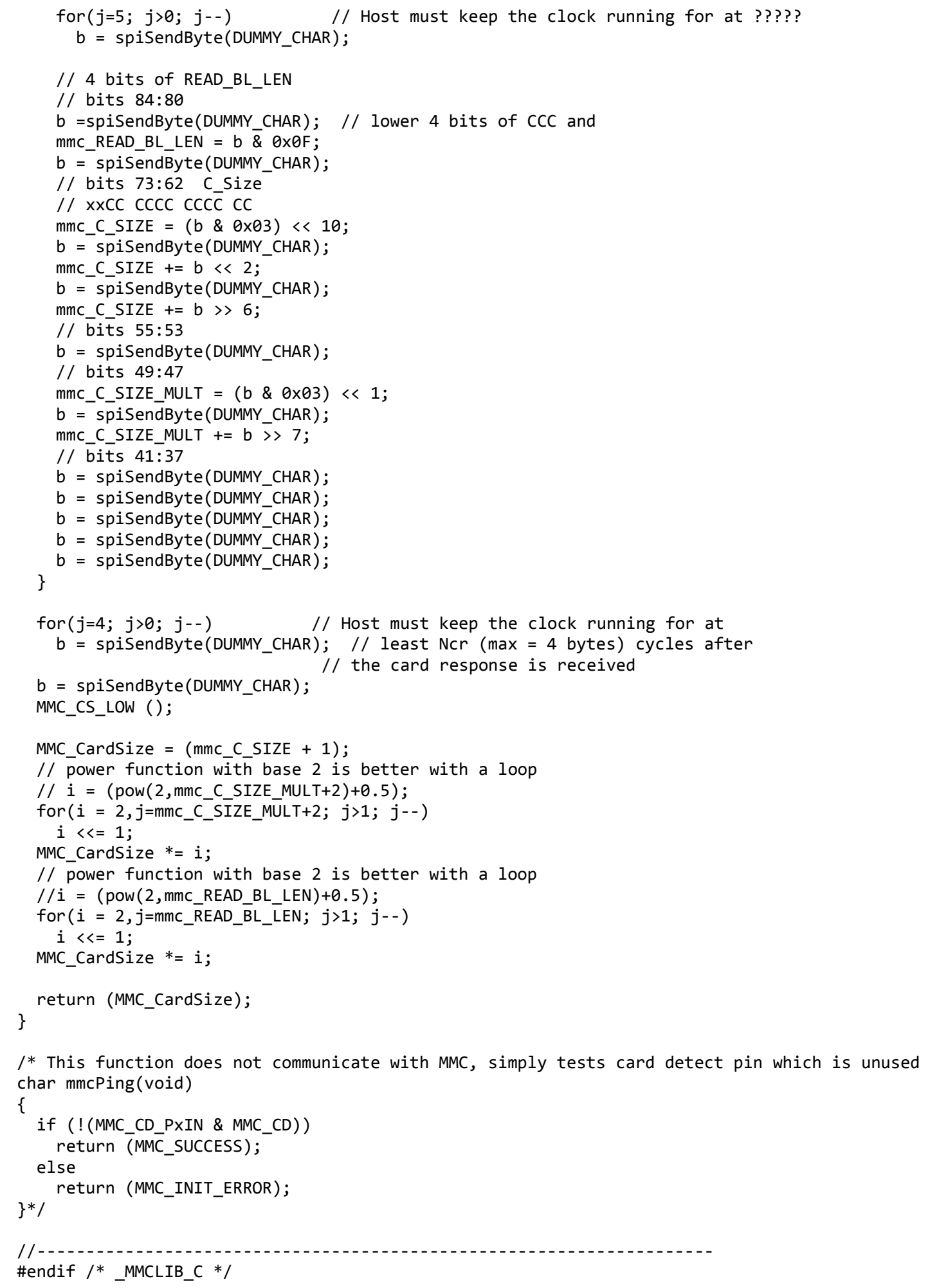

MMC.h

Affiliated MMC communication header file, also ported from TEMPO 3.2 
\#ifndef_MMCLIB_H

\#define_MMCLIB_H

\#include "hal.h"

\#define MMC_INIT_BAUD

UBR_DIV(230000) $\quad / / /<$ Init the MMC card at 230kBaud

\#define MMC_DEF_BAUD

UBR_DIV(2000000) $/ / /<$ Reset the baud rate to 2 Mbaud

\#define MMC_CS_HIGH()

MMC_CS $=1$

$/ / /<$ Raise Chip Select (de-select the

MMC)

\#define MMC_CS_LOW()

\#define DUMMY_CHAR

$M M C \_C S=0$

OxFF

$/ / /<$ Lower Chip Select (select the MMC)

shifts

\#define MMC_RX_BUFF_SIZE 512

// macro defines

\#define HIGH(a) ((a>>8)\&OxFF)

// high byte from word

\#define LOW(a) (a\&0xFF)

// low byte from word

// Tokens (necessary because at NPO/IDLE (and CS active) only Oxff is on the data/command line)

\#define MMC_START_DATA_BLOCK_TOKEN Oxfe // Data token start byte, Start Single Block Read

\#define MMC_START_DATA_MULTIPLE_BLOCK_READ Oxfe // Data token start byte, Start Multiple Block Read

\#define MMC_START_DATA_BLOCK_WRITE - Oxfe // Data token start byte, Start Single Block Write

\#define MMC_START_DATA_MULTIPLE_BLOCK_WRITE Oxfc // Data token start byte, Start Multiple Block Write

\#define MMC_STOP_DATA_MULTIPLE_BLOCK_WRITE Oxfd // Data toke stop byte, Stop Multiple Block Write

// an affirmative R1 response (no errors)

\#define MMC_R1_RESPONSE $\quad$ Ox00

// this variable will be used to track the current block length

$/ /$ this allows the block length to be set only when needed

$/ /$ unsigned long_BlockLength = 0 ;

// error/success codes

\#define MMC_SUCCESS $\quad 0 \times 00$

\#define MMC_BLOCK_SET_ERROR $0 \times 01$

\#define MMC_RESPONSE_ERROR OxO2

\#define MMC_DATA_TOKEN_ERROR $0 \times 03$

\#define MMC_INIT_ERROR ${ }^{-} \quad 0 \times 04$

\#define MMC_CRC_ERROR $\quad 0 \times 10$

\#define MMC_WRITE_ERROR $0 \times 11$

\#define MMC_OTHER_ERROR

\#define MMC_TIMEOUT_ERROR OXFF

// commands: first bit $\theta$ (start bit), second 1 (transmission bit); CMD-number + $\theta$ ffsett $\theta \times 40$

\#define MMC GO IDLE STATE $\quad$ Ox40 //CMDO

\#define MMC_SEND_OP_COND $\quad$ Ox41 //CMD1

\#define MMC READCSD $\quad$ Ox49 //CMD9

\#define MMC_SEND_CID $\quad$ Ox4a $\quad / / C M D 10$

\#define MMC_STOP_TRANSMISSION

\#define MMC_SEND_STATUS $\quad$ 0x4d //CMD13

\#define MMC SET BLOCKLEN

\#define MMC_READ_SINGLE_BLOCK

0x50 //CMD16 set block length for next read/write

\#define MMC_READ_MULTIPLE_BLOCK Ox52 //CMD18

\#define MMC_CMD_WRITEBLOCK Ox54 //CMD20 Write block to memory

\#define MMC WRITE_BLOCK

$0 \times 58 \quad / / C M D 24$

\#define MMC_WRITE_MULTIPLE_BLOCK

\#define MMC_WRITE_CSD $\quad$ Ox5b //CMD27

\#define MMC_SET_WRITE_PROT $\quad$ Ox5C $\quad / / C M D 28$

\#define MMC_CLR_WRITE_PROT

\#define MMC_SEND_WRITE_PROT $\quad 0 \times 5 \mathrm{e} \quad / /$ CMD30

\#define MMC_TAG_SECTOR_START $\quad 0 \times 60 \quad / / C M D 32$

\#define MMC_TAG_SECTOR_END $\quad 0 \times 61 \quad$ //CMD33

\#define MMC_UNTAG_SECTOR $\quad 0 \times 62 \quad / / C M D 34$

\#define MMC_TAG_EREASE_GROUP_START $0 \times 63 \quad / / C M D 35$

\#define MMC_TAG_EREASE_GROUP_END 0x64 //CMD36

\#define MMC_UNTAGG_EREASE_GROÜP

\#define MMC_EREASE $\quad$ Ox66 


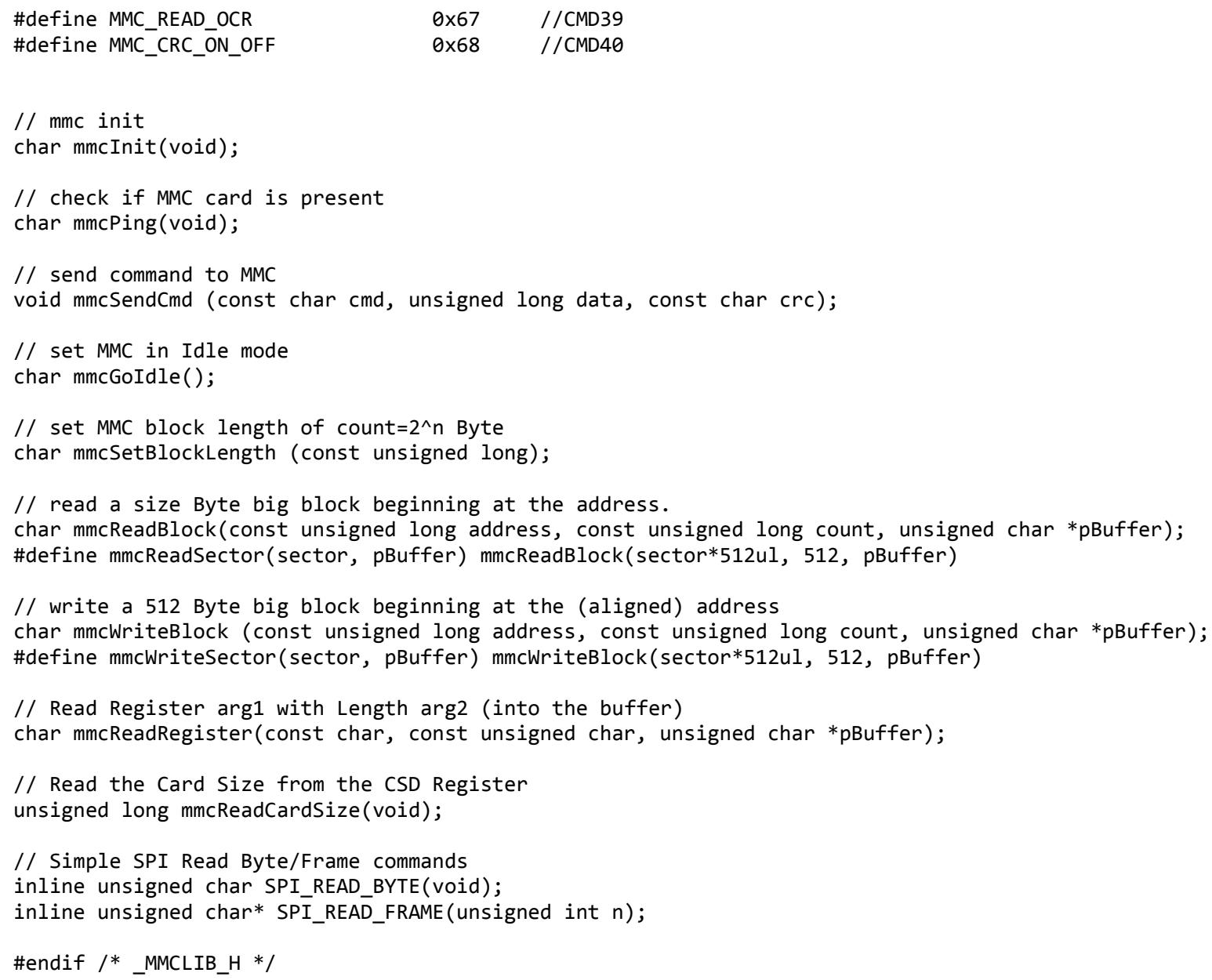

\section{MPU.c}

Simple MPU6xxx series driver, written on top of communications library I2C interface

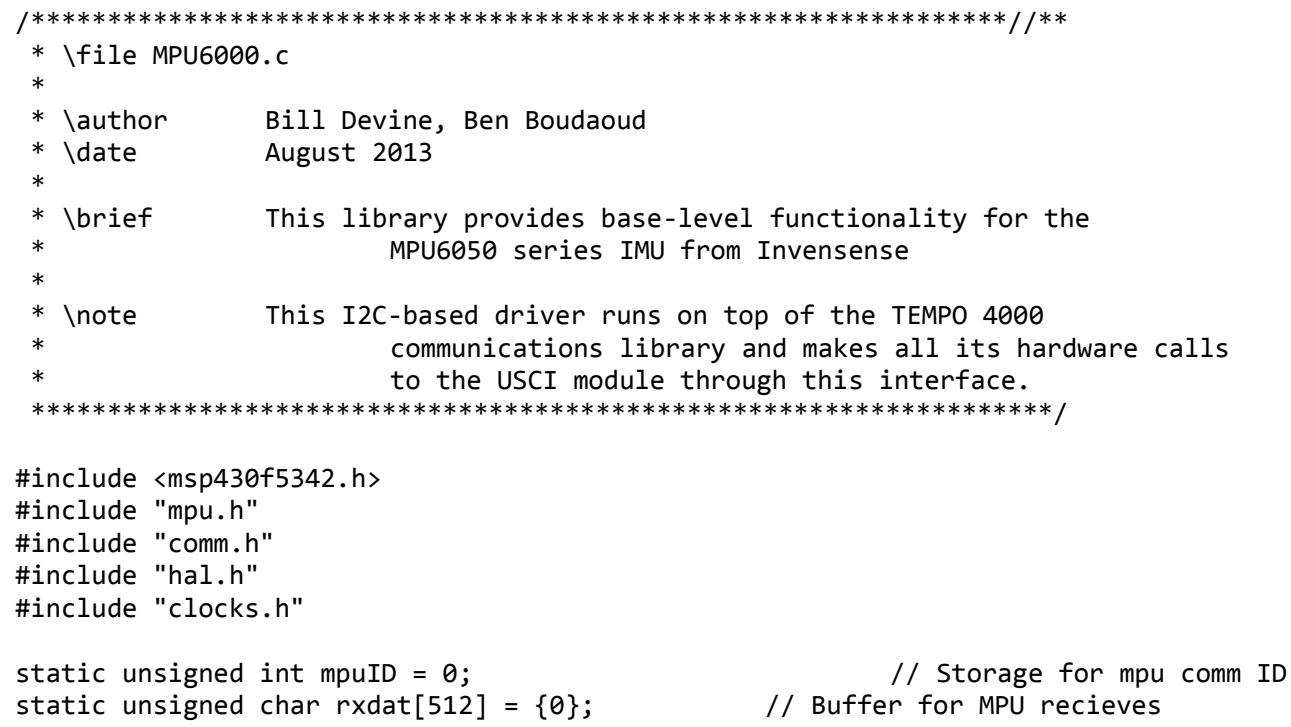




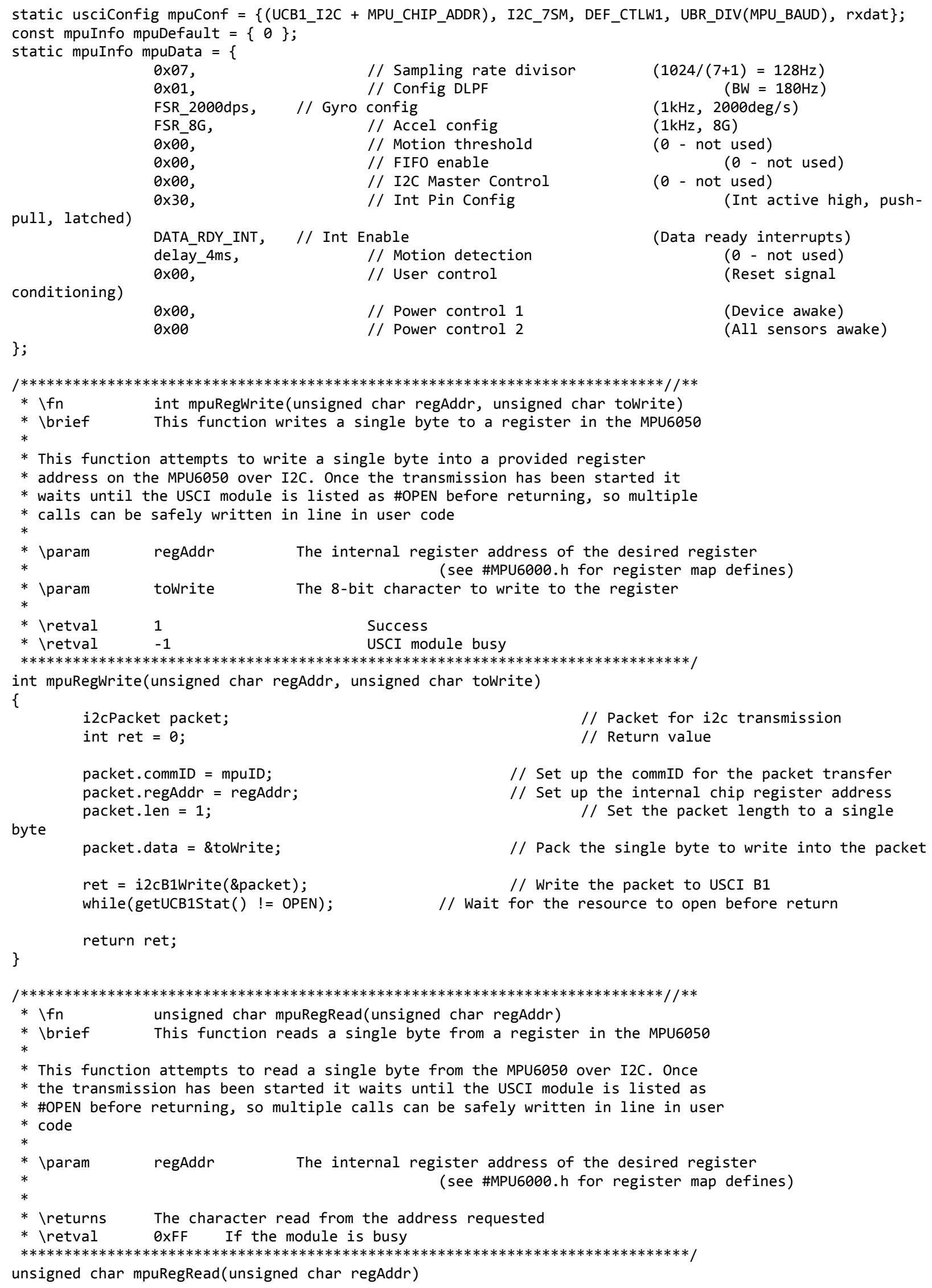




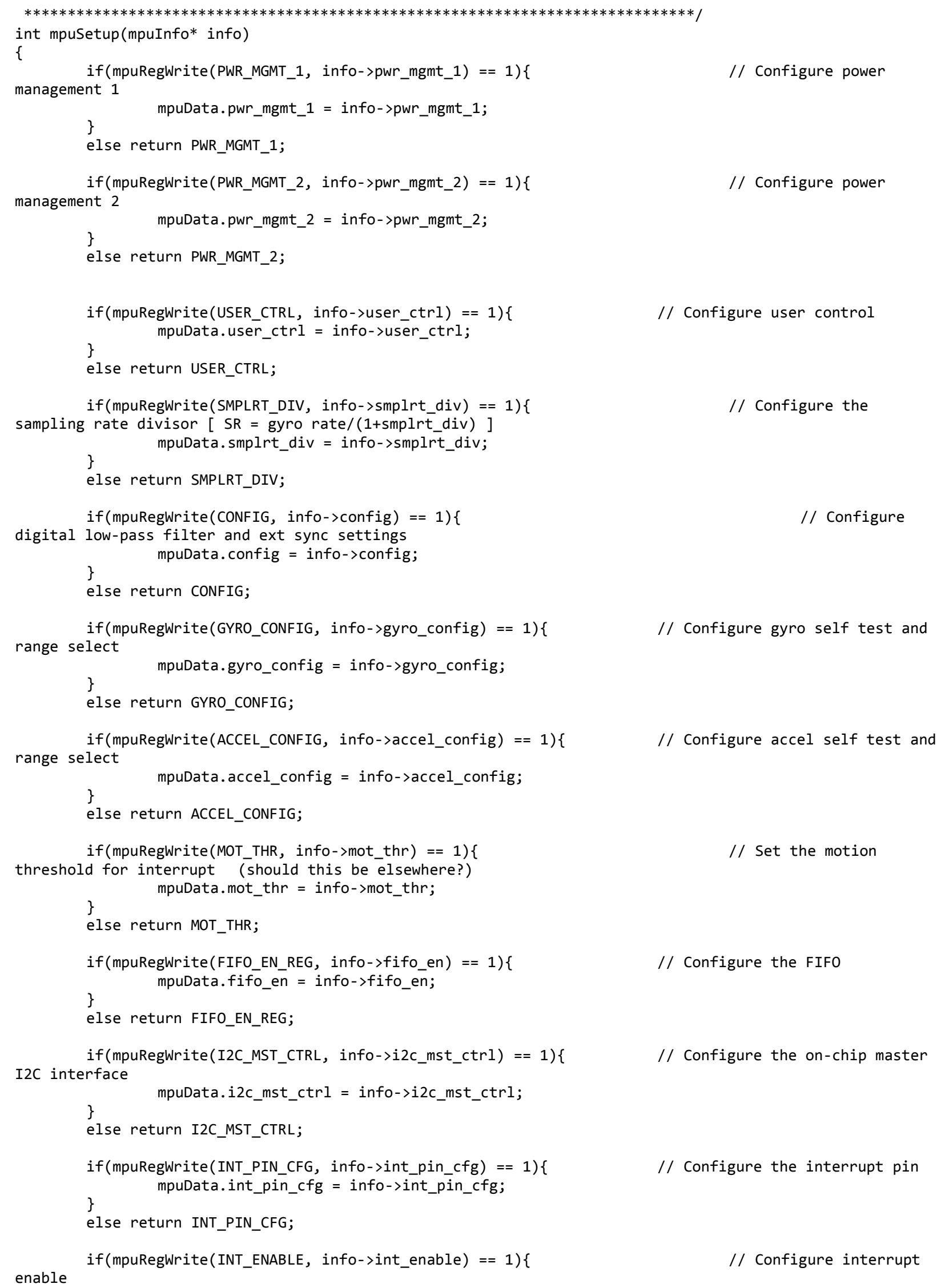




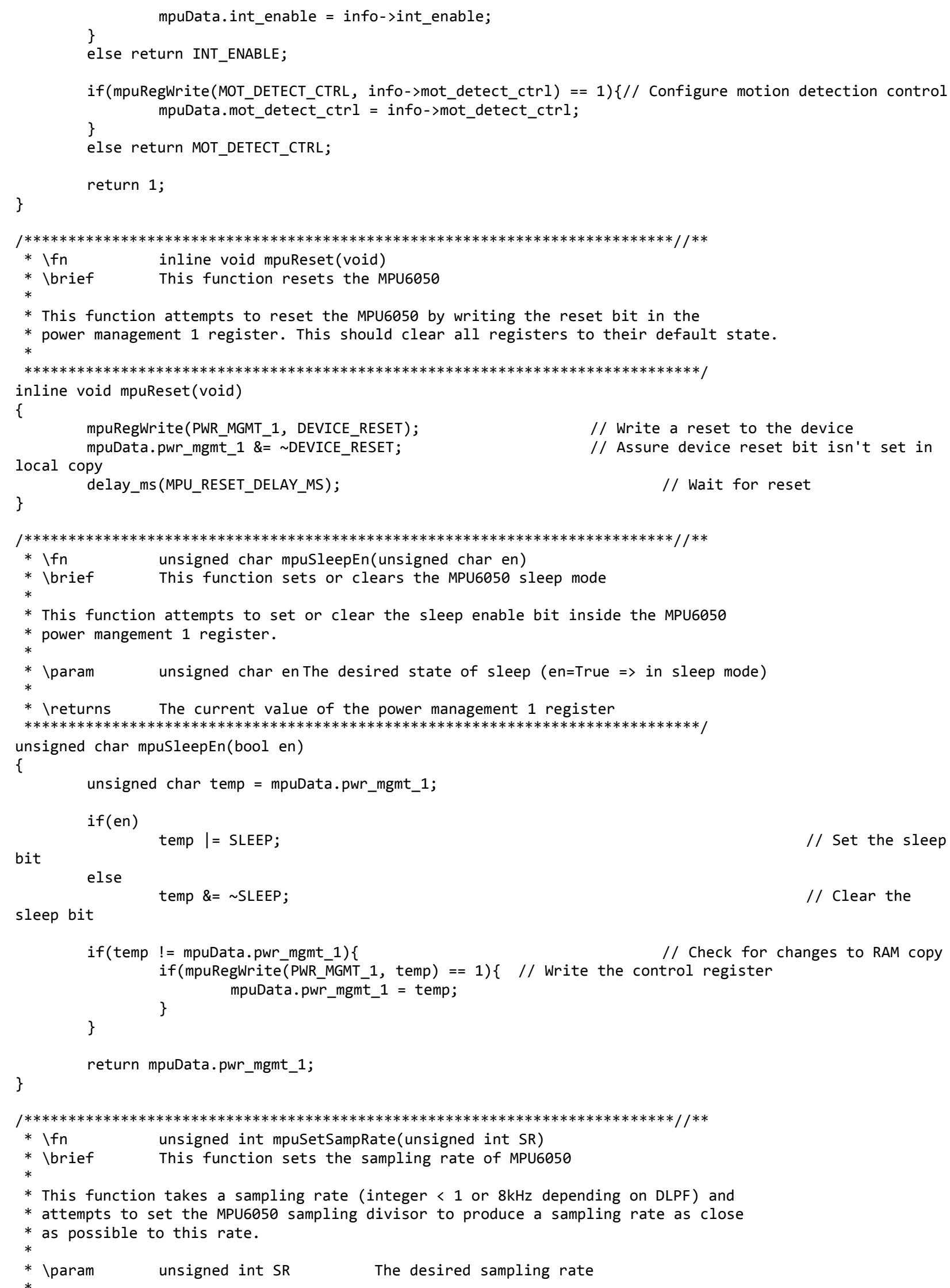




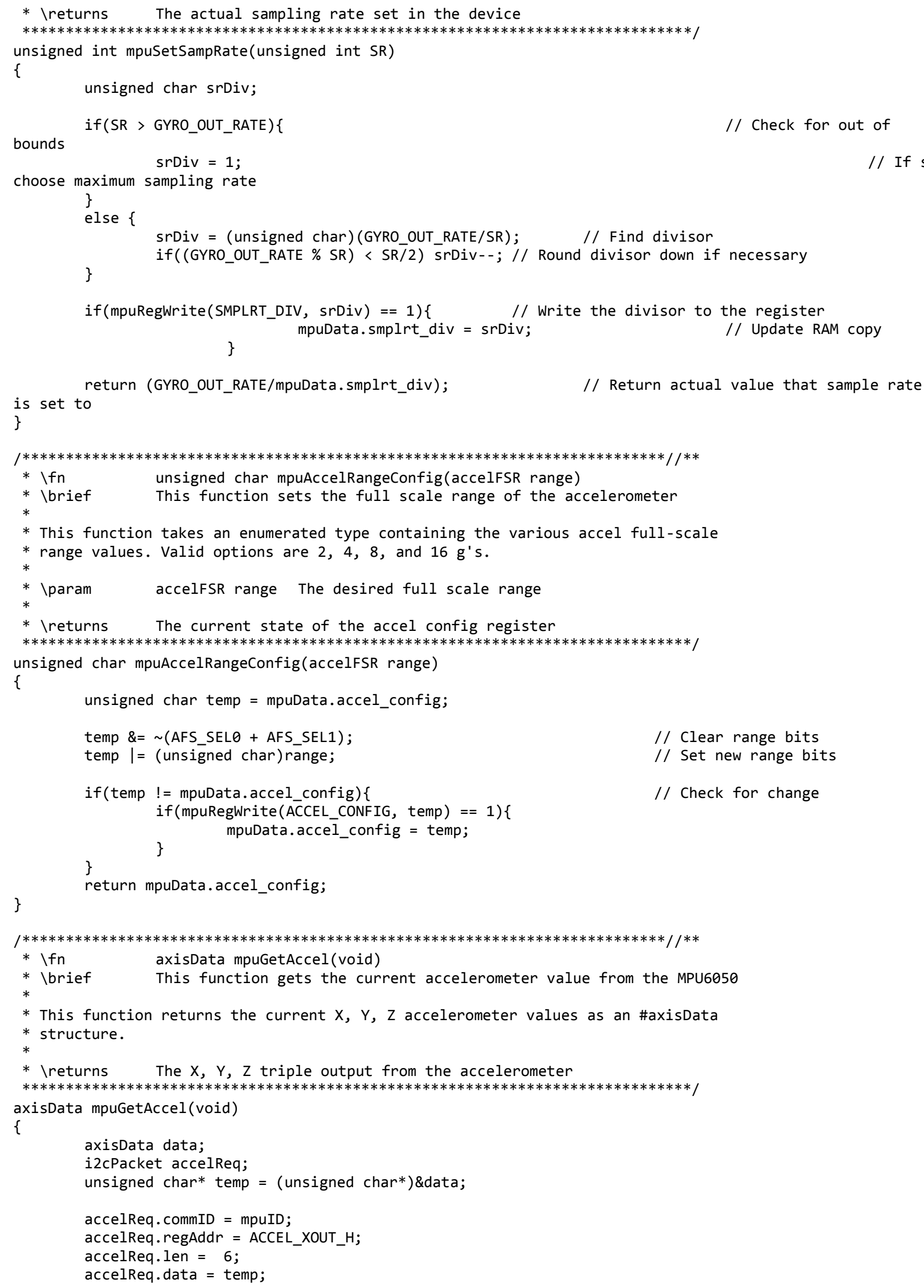




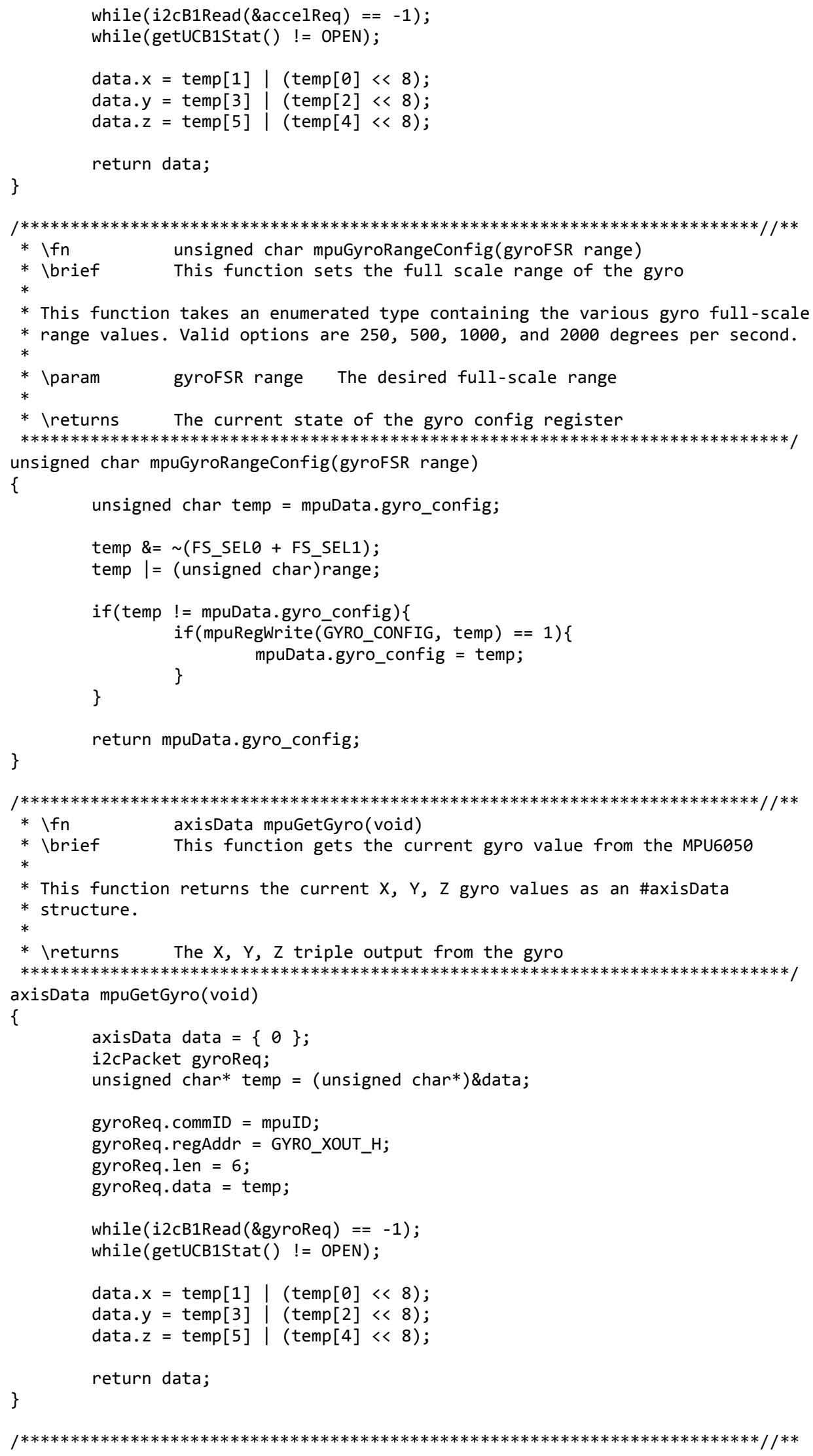




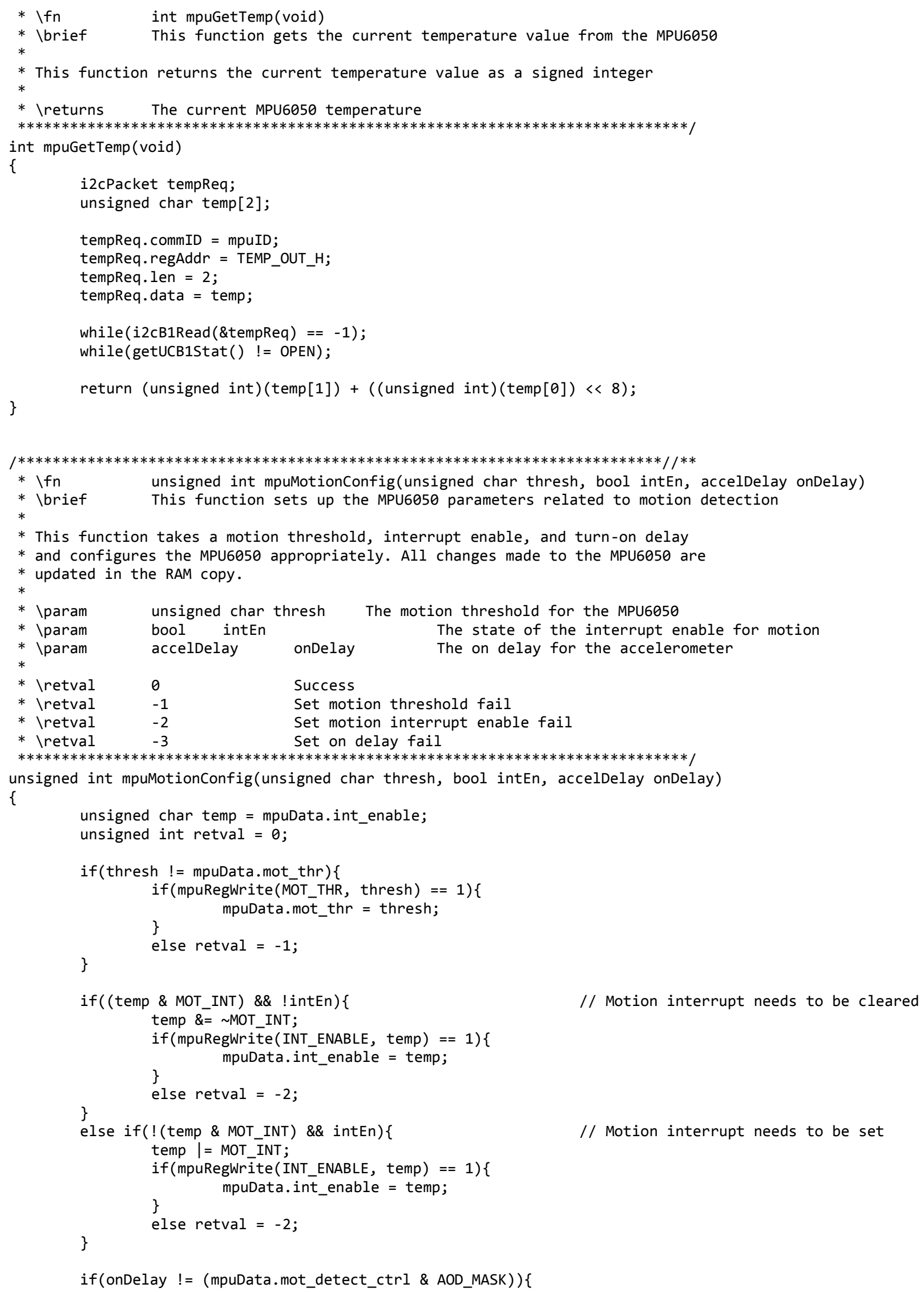




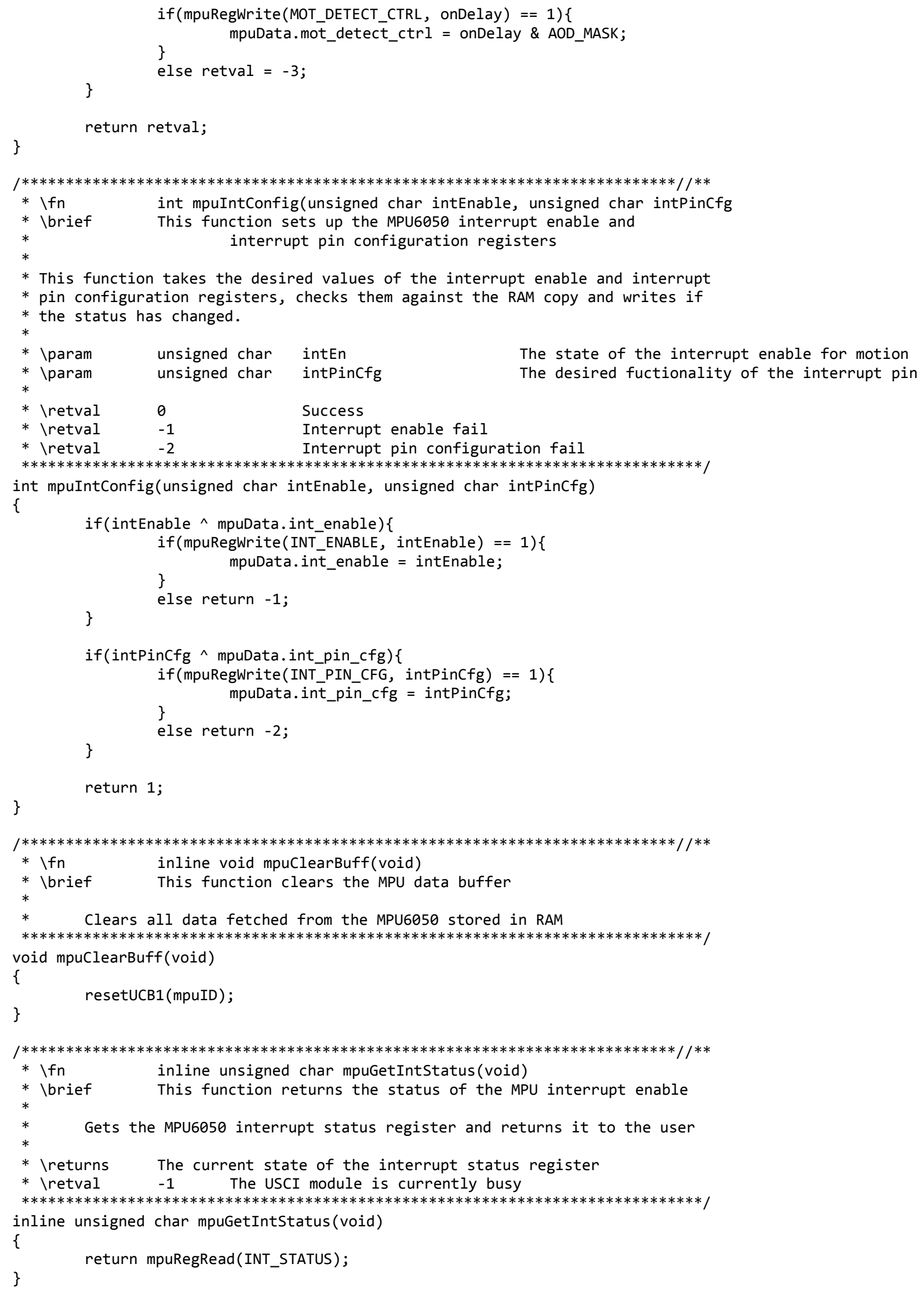




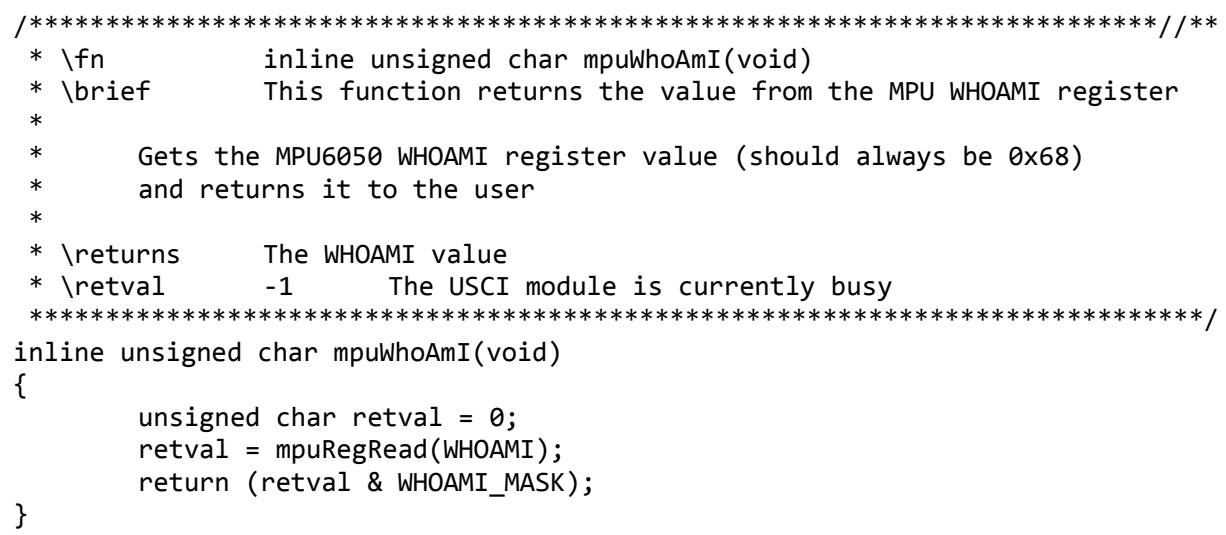

\section{MPU.h}

Affiliated MPU header file, with full register map for MPU series motion capture ICs

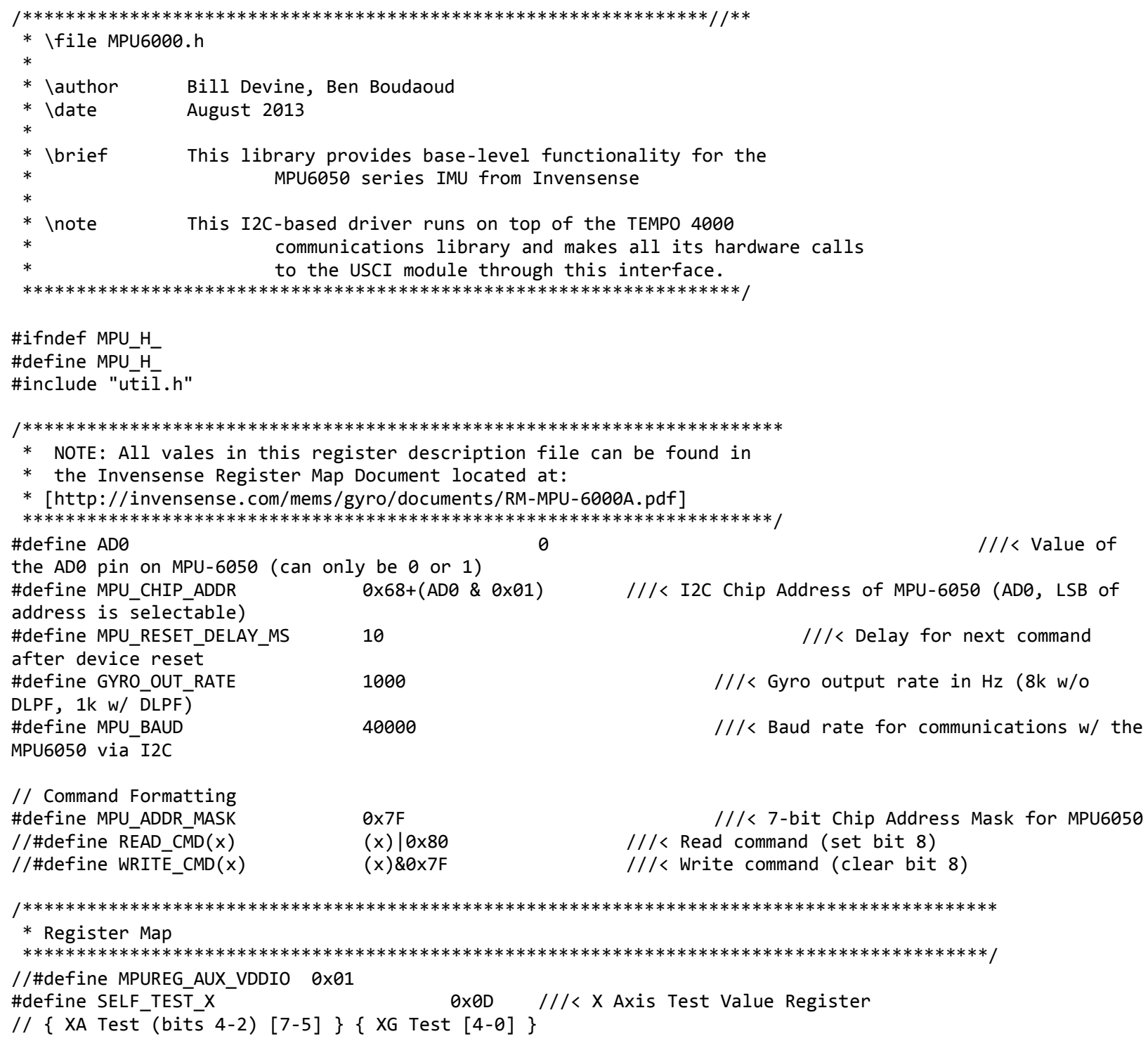

$/ / /<$ Value of ///< I2C Chip Address of MPU-6050 (AD0, LSB of $/ / /<$ Delay for next command $/ / /$ Gyro output rate in $\mathrm{Hz}$ ( $8 \mathrm{k}$ w/o ///< Baud rate for communications $w /$ the /// 7-bit Chip Address Mask for MPU6050 $/ / /<$ Read command (set bit 8 ) $/ / /<$ Write command (clear bit 8 ) 


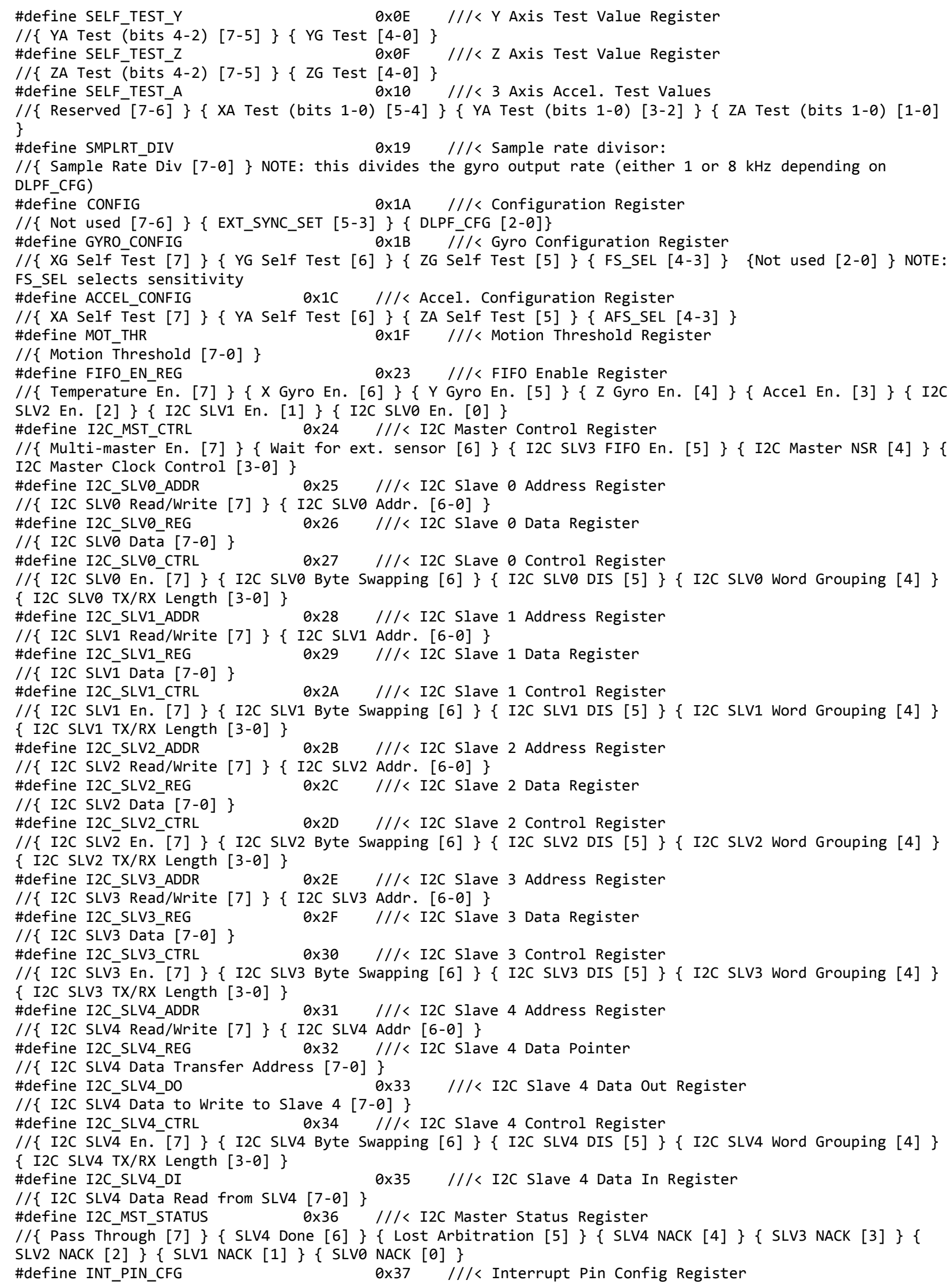


$/ /\{$ Int. Level [7] $\}\{$ Int. Open [6] \} \{ Latch Int. En. [5] \} \{ Int. Read Clear [4] \} \{ FSYNC Int. Level [3] \} \{ FSYNC Int. En. [2] $\}\{$ I2C Bypass En. [1] $\}\{$ Not Used [0] $\}$

\#define INT ENABLE $\quad 0 \times 38 \quad / / /<$ Interrupt Enable Register

$/ /\{$ Not Used [7] $\}\{$ Motion En. [6] $\}\{$ Not Used [5] $\}\{$ FIFO OVFL [4] $\}\{$ I2C MST INT [3] $\}\{$ Not Used

[2-1] \} \{ Data Ready [0] \}

\#define INT_STATUS $\quad 0 \times 3 \mathrm{~A} \quad / / /<$ Interrupt Status Register

$/ /\{$ Not Used [7] $\}\{$ Motion Int. [6] $\}\{$ Not Used [5] $\}\{$ FIFO Int. [4] $\}$ \{ I2C MST INT [3] $\}\{$ Not Used

[2-1] \} \{ Data Ready [0] \}

\#define ACCEL_XOUT_H $0 \times 3 B \quad / / /<\times$ Accel. High Byte Register

$/ /\{X$ Accel. Value High Byte [7-0] \}

\#define ACCEL_XOUT_L

$/ /\{$ X Accel. Value Low Byte [7-0] \}

\#define ACCEL_YOUT_H

$/ /\{$ Y Accel. Value High Byte [7-0] \}

\#define ACCEL_YOUT_L

$/ /\{$ Y Accel. Value Low Byte [7-0] \}

\#define ACCEL ZOUT H Ox3F

$/ /\{$ Z Accel. Value High Byte [7-0] \}

\#define ACCEL_ZOUT_L

$/ / /<x$ Accel. Low Byte Register

$/ / /<Y$ Accel. High Byte Register

$/ / /<Y$ Accel. Low Byte Register

$/ / /<$ Z Accel. High Byte Register

$/ /\{$ Z Accel. Value Low Byte [7-0] \}

\#define TEMP_OUT_H

//\{ Temperature Value High Byte [7-0] \}

\#define TEMP_OUT_L

$/ /\{$ Temperature Value Low Byte [7-0] \}

\#define GYRO_XOUT_H

$/ /\{X$ Gyro Value High Byte [7-0] \}

\#define GYRO_XOUT_L

$/ /\{X$ Gyro Value Low Byte [7-0] $\}$

\#define GYRO_YOUT_H

//\{ Y Gyro Value High Byte [7-0] \}

\#define GYRO_YOUT_L

$/ /\{$ Y Gyro Value Low Vyte [7-0] $\}$

\#define GYRO_ZOUT_H

//\{ Z Gyro Value High Byte [7-0] \}

\#define GYRO_ZOUT_L

$/ /\{$ Z Gyro Value Low Byte [7-0] \}

\#define EXT_SENS_DATA_00 0x49 $/ / /<$ External Sensor Data Register 0

$/ /\{$ Value $[\overline{7}-0] \overline{\}}$

\#define EXT_SENS_DATA_01 $0 \times 4$ A

$/ /\{$ Value $[\overline{7}-\theta] \overline{\}}$

\#define EXT_SENS_DATA_02 0x4B

$/ /\{$ Value $[\overline{7}-\theta] \overline{\}}$

\#define EXT_SENS_DATA_03 0x4C

$/ /\{$ Value $[\overline{7}-0] \overline{\}}$

\#define EXT_SENS_DATA_04 0x4D

$/ /\{$ Value $[\overline{7}-\theta] \overline{\}}$

\#define EXT_SENS_DATA_05 0x4E

$/ /\{$ Value $[\overline{7}-0] \overline{\}}$

\#define EXT_SENS_DATA_06 0x4F

$/ /\{$ Value $[\overline{7}-\theta] \overline{\}}$

\#define EXT_SENS_DATA_07 0x50

$/ /\{$ Value $[\overline{7}-\theta] \overline{\}}$

\#define EXT_SENS_DATA_08 0x51

$/ /\{$ Value $[\overline{7}-\theta] \overline{\}}$

\#define EXT_SENS_DATA_09 $0 \times 52$

$/ /\{$ Value $[\overline{7}-\theta] \overline{\}}$

\#define EXT SENS DATA $100 \times 53$

$/ /\{$ Value $[\overline{7}-\theta] \overline{\}}$

\#define EXT_SENS_DATA_11 0x54

$/ /\{$ Value $[\overline{7}-\theta] \overline{\}}$

\#define EXT_SENS_DATA_12 0x55

$/ /\{$ Value $[\overline{7}-\theta] \overline{\}}$

\#define EXT_SENS_DATA_130x56

$/ /\{$ Value $[\overline{7}-0] \overline{\}}$

\#define EXT_SENS_DATA_14 0x57

$/ /\{$ Value $[\overline{7}-\theta] \overline{\}}$

\#define EXT_SENS_DATA_15 0x58

$/ /\{$ Value $[\overline{7}-\theta] \overline{\}}$

\#define EXT_SENS_DATA_16 0x59

///< External Sensor Data Register 1

$/ / /<$ External Sensor Data Register 2

$/ / /<$ External Sensor Data Register 3

$/ / /<$ External Sensor Data Register 4

$/ / /<$ External Sensor Data Register 5

$/ / /<$ External Sensor Data Register 6

$/ / /<$ External Sensor Data Register 7

$/ / /<$ External Sensor Data Register 8

$/ / /<$ External Sensor Data Register 9

$/ / /<$ External Sensor Data Register 10

///< External Sensor Data Register 11

///< External Sensor Data Register 12

///< External Sensor Data Register 13

$/ / /<$ External Sensor Data Register 14

$/ / /<$ External Sensor Data Register 15

$/ / /<$ External Sensor Data Register 16 
$/ /\{$ Value [7-0] $\}$

\#define EXT_SENS_DATA_17 0x5A

$/ /\{$ Value $[\overline{7}-0] \overline{\}}$

\#define EXT_SENS_DATA_18 0x5B

$/ /\{$ Value $[\overline{7}-0] \overline{\}}$

\#define EXT_SENS_DATA_19005C

$/ /\{$ Value $[\overline{7}-\theta]\}$

\#define EXT_SENS_DATA_20 0x5D

$/ /\{$ Value $[\overline{7}-0]\}$

\#define EXT_SENS_DATA_21 0x5E

$/ /\{$ Value $[\overline{7}-\theta]\}$

\#define EXT_SENS_DATA_22 0x5F

$/ /\{$ Value $[\overline{7}-\theta]\}$

\#define EXT_SENS_DATA_23 0x60

$/ /\{$ Value $[\overline{7}-\theta]\}$

\#define I2C_SLVO_DO

$/ /\{$ I2C SLVo Data Out [7-0] \}

\#define I2C_SLV1_DO

$/ /\{$ I2C SLV 1 Data Out [7-0] \}

\#define I2C_SLV2_DO

$/ /\{$ I2C SLV2 Data Out [7-0] $\}$

\#define I2C_SLV3_DO

$/ /\{$ I2C SLV 3 Data Out [7-0] $\}$

\#define I2C_MST_DELAY_CTRL

$/ / /<$ External Sensor Data Register 17

$/ / /<$ External Sensor Data Register 18

$/ / /<$ External Sensor Data Register 19

$/ / /<$ External Sensor Data Register 20

$/ / /<$ External Sensor Data Register 21

$/ / /<$ External Sensor Data Register 22

$/ / /<$ External Sensor Data Register 23

Ox67 $/ / /<$ I2C Master Delay Control Reg

$/ /\{$ Delay Shadow En. [7] \} \{ Not Used [6-5] \} \{ I2C SLV4 Delay [4] \} \{ I2C SLV3 Delay [3] $\}$ I2C SLV2

Delay [2] $\}\{$ I2C SLV1 Delay [1] $\}\{$ I2C SLV0 Delay [0] $\}$

\#define SIGNAL_PATH_RESET Ox68 ///< Signal Path Reset Register

$/ /\{$ Not Used [7-3] $\}\{$ Gyro Reset [2] $\}\{$ Accel. Reset [1] \} \{ Temp Reset [0] \}

\#define MOT_DETECT_CTRL $\quad 0 \times 69 \quad / / /<$ Motion Detection Control Reg

$/ /\{$ Not Used [7-6] $\}\{$ Accel. Power-on Delay [5-4] $\}$ \{ Not Used [3-0] \}

\#define USER_CTRL

$0 \times 6 \mathrm{~A} \quad / / /<$ User Control Register

$/ /\left\{\right.$ Not Used [7] $_{\text {[ }}\{$ FIFO En. [6] $\}\{$ I2C Master En. [5] $\}\{$ I2C IF DIS [4] $\}\{$ Not Used [3] $\}\{$ FIFO

Reset [2] \} \{I2C Master Reset [1] \} \{ Signal Cond. Reset [0] \}

\#define PWR_MGMT_1 Ox6B $/ / /<$ Power Management Register 1

$/ /\{$ Device Reset [7] $\}\{$ Sleep [6] $\}\{$ Cycle [5] $\}\{$ Not Used [4] \} \{ Temp. Disable [3] \} \{ Clock Select $[2-0]\}$

\#define PWR_MGMT_2 $\quad$ 0x6C $\quad / / /<$ Power Management Register 2

$/ /\{$ Low-power wake control [7-6] \} \{ Standby X Accel [5] \} \{ Standby Y Accel [4] \} \{ Standby Z Accel. [3]

\} \{ Standby X Gyro [2] \} \{ Standby Y Gyro [1] \} \{ Standby Z Gyro [0] \}

\#define FIFO_COUNTH $\quad 0 \times 72 \quad / / /<$ FIFO Size High Byte Register

$/ /\{$ FIFO Size Value High Byte [7-0] $\}$

\#define FIFO_COUNTL

$/ /\{$ FIFO Size Value Low Byte [7-0] \}

\#define FIFO_R_W

$/ /\{$ FIFO Value $[7-0]\}$

\#define WHOAMI

$0 \times 74$

0x73 $/ / /<$ FIFO Size Low Byte Register

$/ /\{$ Not Used [7] \} \{ Upper 5 bits of I2C Address [6-1] $\{$ Not Used [0]

// NOTE: the least-significant bit of the device I2C address is provided by the status of the ADO pin if the MPU6050 is used (this is not reflected in WHOAMI)

\begin{tabular}{|c|c|c|}
\hline \multicolumn{3}{|l|}{ //CONFIG } \\
\hline \#define EXT_SYNC_SETO & $0 \times 00$ & $/ / /<$ FSYNC Disabled \\
\hline \#define EXT_SYNC_SET1 & $0 \times 08$ & $/ / /<$ FSYNC on low bit of TEMP_OUT_L \\
\hline \#define EXT_SYNC_SET2 & $0 \times 10$ & $/ / /<$ FSYNC on low bit of GYRO_XOUT_L \\
\hline \#define EXT_SYNC_SET3 & $0 \times 18$ & $/ / /<$ FSYNC on low bit of GYRO_YOUT_L \\
\hline \#define EXT_SYNC_SET4 & $0 \times 20$ & $/ / /<$ FSYNC on low bit of GYRO_ZOUT_L \\
\hline \#define EXT_SYNC_SET5 & $0 \times 28$ & $/ / /<$ FSYNC on low bit of ACCEL_XOUT_L \\
\hline \#define EXT_SYNC_SET6 & $0 \times 30$ & $/ / /<$ FSYNC on low bit of ACCEL_YOUT_L \\
\hline \#define EXT_SYNC_SET7 & $0 \times 38$ & $/ / /<$ FSYNC on low bit of ACCEL_ZOUT_L \\
\hline $\begin{array}{l}\text { \#define DLPF_CFG0 } \\
\text { notes. }\end{array}$ & $0 \times 00$ & $/ / /<$ DLPF on setting 0 . Check Register Description document for \\
\hline $\begin{array}{l}\text { \#define DLPF_CFG1 } \\
\text { notes. }\end{array}$ & $0 \times 01$ & $/ / /<$ DLPF on setting 0 . Check Register Description document for \\
\hline \#define DLPF_CFG2 & $0 \times 02$ & $/ / /<$ DLPF on setting 0 . Check Register Description document for \\
\hline
\end{tabular}




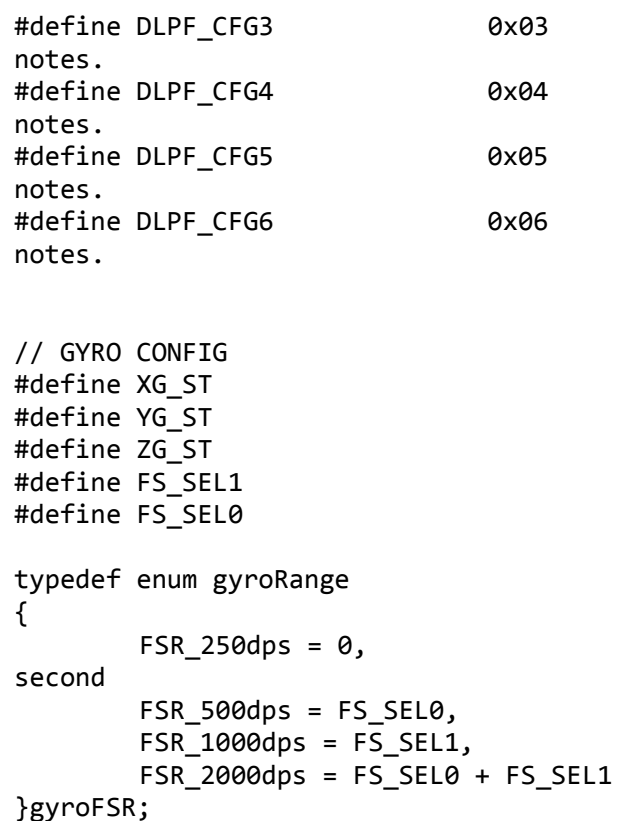

// ACCEL_CONFIG

\#define XA_ST

\#define YA_ST

\#define ZA_ST

\#define AFS_SEL1

\#define AFS_SELO

typedef enum accelerometerRange

\{

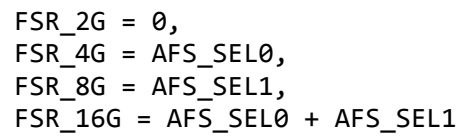

0x80 $\quad / / /<$ Enable multi-master capability

0x04 $\quad / / /<$ Delay data ready interrupt until

$/ / /<$ Enables EXT_SENS_DATA for SLV3 to be written to FIFO

$/ / /<0$ : reset occurs between slave reads, 1 : stop and

$/ / /<$ I2C Clk Rate divisor mask (SEE REGISTER MAP PAGE 19 FOR

0x80 $/ / /<$ I2C Read/Write Control (1: Read, 0 : Write) $/ / /<7$ Bit slave address field

0x80 $\quad / / /<$ Enable slave device for data transfers $/ / /<$ Enable byte swapping (high and low byte order) $/ / /<1$ : Read/write only, 0 : write before read 
\#define I2C SLV GRP a word, $1: \overline{1}$ and 2 form a word) \#define I2C_SLV_LEN_MASK $0 x \theta F$
$0 \times 10$

$/ / /<$ Byte pairing for word order $(0: 0$ and 1 form

$/ / /<$ Number of bytes transferred to/from slave device

// I2C MASTER STATUS

\#define PASS_THROUGHT 0x80 $\quad / / /<$ Status of FSYNC interrupt from an external device (interrupt triggered if FSYNC_INT_EN asserted in INT_PIN_CFG)

\#define I2C_SLV4_DONE

$/ / \overline{<}$ Set to 1 when Slave 4 completes transmit (interrupt triggered if SLV4 DONE INT asserted in I2C SLV4 CTRL)

\#define I2C_LOST_ARB $\quad 0 \times 20 \quad-/ / /$ Set to 1 when I2C master loses arbitration of the bus (interrupt triggered if I2C_MST_INT_EN asserted in INT_ENABLE)

\#define I2C_SLV4_NACK $\quad$ ox10 $\quad / / /$ S Set when I2C master receives a NACK from slave 4

(interrupt triggered if I2C_MST_INT_EN asserted in INT_ENABLE)

\#define I2C_SLV3_NACK $\quad 0 x 08 \quad / / /<$ Same for slave 3

\#define I2C_SLV2_NACK $\quad 0 \times 04 \quad / / /<$ Same for slave 2

\#define I2C_SLV1_NACK $\quad 0 \times 02 \quad 1 / /<$ Same for slave 1

\#define I2C_SLVO_NACK $\quad 0 \times 01 \quad 1 / /<$ Same for slave $\theta$

// INTERRUPT PIN CONFIG

\#define INT_LEVEL

\#define INT_OPEN

0x80 $\quad / / /<$ Current status of the INT pin

configured as open-drain

\#define LATCH_INT_EN

held high until cleared

\#define INT_RD_CLEAR

INT_STATUS, 1: Interrupt bits cleared on any read

\#define FSYNC_INT_LEVEL

\#define FSYNC_INT_EN

interrupts

\#define I2C_BYPASS_EN

$/ / /<\theta$ : INT pin configured as push-pull, 1: INT pin

$0 \times 20$

$/ / /<0$ : INT pin emits a 50us long pulse, 1 : INT pin is

0x02

$/ / /<0$ : Interrupt status bits are cleared only by reading

$0 \times 10$ $/ / /<$

0x08 $\quad / / /<0$ : FSYNC is active high, 1 : FSYNC is active low

0x04 $/ / /<0$ : Disable FSYNC interrupts, 1: Enable FSYNC

I2C_MST_EN is $\theta$ the host processor can directly access the auxiliary I2C bus

// INTERRUPT ENABLE/STATUS (write to INT ENABLE, read from INT STATUS)

\#define MOT_INT

\#define FIFO_OFLOW_INT

\#define I2C_MST_INT_INT

\#define DATA_RDY_INT

// I2C MASTER DELAY CONTROL \#define DELAY_ES_SHADOW

until all data has been RX'd

\#define I2C_SLV4_DLY_EN

\#define I2C_SLV3_DLY_EN

\#define I2C_SLV2_DLY_EN

\#define I2C_SLV1_DLY_EN

\#define I2C_SLVO_DLY_EN

// SIGNAL PATH RESET

\#define GYRO_RESET

\#define ACCEL_RESET

\#define TEMP_RESET

0x10
0x08
0x01

$/ / /<$ Enable motion detection interrupt bit

$/ / /<$ Enable FIFO overflow interrupt generation

$/ / /<$ Enable I2C interrupt sources

$/ / /<$ Enable sensor register write interrupt

0x80 $\quad / / /<$ When set delays shadowing of external sensor data

0x10 $\quad / / /<$ Slave 4 only accessed at a decreased rate

0x08 $\quad / / /<$ Same as \#I2C_SLV4_DLY_EN for slave 3

0x04 $\quad / / /<$ Same as \#I2C_SLV4_DLY_EN for slave 2

0x02 $\quad / / /<$ Same as \#I2C_SLV4_DLY_EN for slave 1

0x01 $\quad / / /<$ Same as \#I2C_SLV4_DLY_EN for slave $\theta$

0x04 $\quad / / /<$ Reset analog and digital gyro signal paths 0x02 $\quad / / /<$ Reset analog and digital accel signal paths 0x01 $\quad / / /<$ Reset analog and digital temp signal paths

// MOTION DETECTION CONTROL

// NOTE: accelerometer has a default start-up delay of $4 \mathrm{~ms}$, these bits can be used to extend it up to $7 \mathrm{~ms}$

\#define ACCEL_ON DELAY1

\#define ACCEL_ON_DELAYO

$0 \times 20 \quad / / /<$ Accel. power on additional delay bit 1

\#define AOD_MASK

0x10 $\quad / / /<$ Accel. power on additional delay bit $\theta$ ACCEL_ON_DELAYO + ACCEL_ON_DELAY1

$/ / /$ < Mask for acceleromter on

delay bits

typedef enum accelonDelay

/// Accel on delay enumerated type

\{

delay_4ms $=0$,

$4 \mathrm{~ms}$ total ( $+\theta$ extra ms)

extra ms)

delay_5ms = ACCEL_ON_DELAYO,

$/ / /<$ On delay of

extra ms)

delay_6ms = ACCEL_ON_DELAY1,

$/ / /<$ On delay of $5 \mathrm{~ms}$ total $(+1$

delay_7ms $=$ ACCEL_ON_DELAYO + ACCEL_ON_DELAY1

$/ / /<$ On delay of $6 \mathrm{~ms}$ total $(+2$

$/ / /<$ On delay of $7 \mathrm{~ms}$ total (+3 extra ms) 
\} accelDelay;

// USER CONTROL

// NOTE: for MPU6000 the primary SPI interface will be enabled in place of primary I2C when I2C_IF_DIS is 1

\#define FIFO_EN

\#define I2C_MST_EN

\#define I2C_IF_DIS

write as 0

\#define FIFO_RESET

clears to $\theta$ on reset complete)

\#define I2C_MST_RESET

$\theta$ on reset complete)

\#define SIG_COND_RESET

$\theta$ on reset complete)

// POWER MANAGEMENT 1

\#define DEVICE RESET

clears to $\theta$ on reset complete)

\#define SLEEP

\#define CYCLE

wake to take a single sample from each sensor $\begin{array}{ll}\text { 0x04 } & / / /<\text { Enable FIFO operation } \\ \text { 0x02 } & / / /<\text { Enable I2C Master mode }\end{array}$

0x01 $/ / /<$ MPU6000: Disable I2C and enable SPI

$/ / /<$ MPU6050: Always

0x04 $\quad / / /<$ Reset FIFO buffer when FIFO_EN $=0$ (auto-

0x02 $/ / /<$ Reset I2C Master when I2C_MST_EN $=0$ (auto-clears to

0x01 $\quad / / /<$ Resets the signal path for all sensor (auto-clear to

0x80 $\quad / / /<$ Reset all internal registers to defaults (auto-

0x40 $\quad / / /<$ Enter sleep mode

$/ / /<$ When set and SLEEP cleared, the device will

$/ / /<$ NOTE: the rate of

this wake is determined by the value of LP_WAKE_CTRL

\#define TEMP_DIS

0x08

$/ / /<$ Disables the temperature sensor

\#define CLKSELL2

$0 \times 04$

$/ / /<$ Clock source selection bit 2

\#define CLKSEL1

\#define CLKSELO

// Operating clock freq enumeration

0x02 $\quad / / /<$ Clock source selection bit 1

\#define CLK SEL 8MHZ $0 \times 00$

\#define CLK_SEL_XG_PLL $\quad 0 \times 01$

\#define CLK_SEL_YG_PLL $\quad 0 \times 02$

\#define CLK_SEL_ZG_PLL

\#define CLK_SEL_EXT32_PLL

reference

\#define CLK_SEL_EXT19_PLL 0x05

reference

\#define CLK_SEL_STOP $\quad 0 \times 07$

$/ / /<$ Clock source selection bit $\theta$

reset

$/ / /<$ Select the internal $8 \mathrm{MHz}$ oscillator for operation

$/ / /<$ Use the internal $\mathrm{PLL}$ with $\mathrm{X}$ gyro reference

$/ / /<$ Use the internal PLL with $Y$ gyro reference

$/ / /<$ Use the internal PLL with $Z$ gyro reference

$/ / /<$ Use the internal PLL with external $32.768 \mathrm{kHz}$ crystal

// POWER MANAGEMENT 2

\#define LP_WAKE_CTRL1

\#define LP_WAKE_CTRLO

$0 \times 80$

$/ / /<$ Use the internal PLL with external 19.2MHz crystal

$0 \times 40$

$/ / /<$ Stop the clock and keep the timing generator in

\#define STBY_XA

\#define STBY_YA

\#define STBY_ZA

\#define STBY_XG

\#define STBY YG

\#define STBY_ZG

\#define LP_WAKE_1.25HZ

\#define LP_WAKE_5HZ

\#define LP_WAKE_2OHZ

\#define LP_WAKE_ $40 \mathrm{HZ}$

$0 \times 20$

$0 \times 10$

$0 \times 08$

$0 \times 04$

$0 \times 02$

$0 \times 01$

CYCLE bit

$0 \times 00$

$0 \times 02$

$0 \times 03$

// WHO AM I

\#define WHOAMI_MASK

\#define WHOAMI_VAL

typedef struct mpuInformation

\{

unsigned char smplrt_div;

unsigned char config;

unsigned char gyro_config;

unsigned char acce $\bar{l}_{\text {_config; }}$

unsigned char mot_thr;

unsigned char fifo_en;

unsigned char i2c_mst_ctrl; $/ / /<$ Low-power wake up at $5 \mathrm{~Hz}$

$/ / /<$ Low-power wake up at $20 \mathrm{~Hz}$

$/ / /<$ Low-power wake up at $40 \mathrm{~Hz}$

0x7E $\quad / / /<$ Mask for 6 bit I2C address of MPU-60X0

0x68 $\quad / / /<$ WWHOAMI value from register

/// Typedef structure for MPU6050 state control

$/ / /<$ Sample rate divisor

$/ / /<$ Chip configuration

$/ / /<$ Gyro configuration

$/ / /<$ Accel configuration

$/ / /<$ Motion threshold

$/ / /<$ Fifo enable

$/ / /<$ MPU6050 I2C master control 


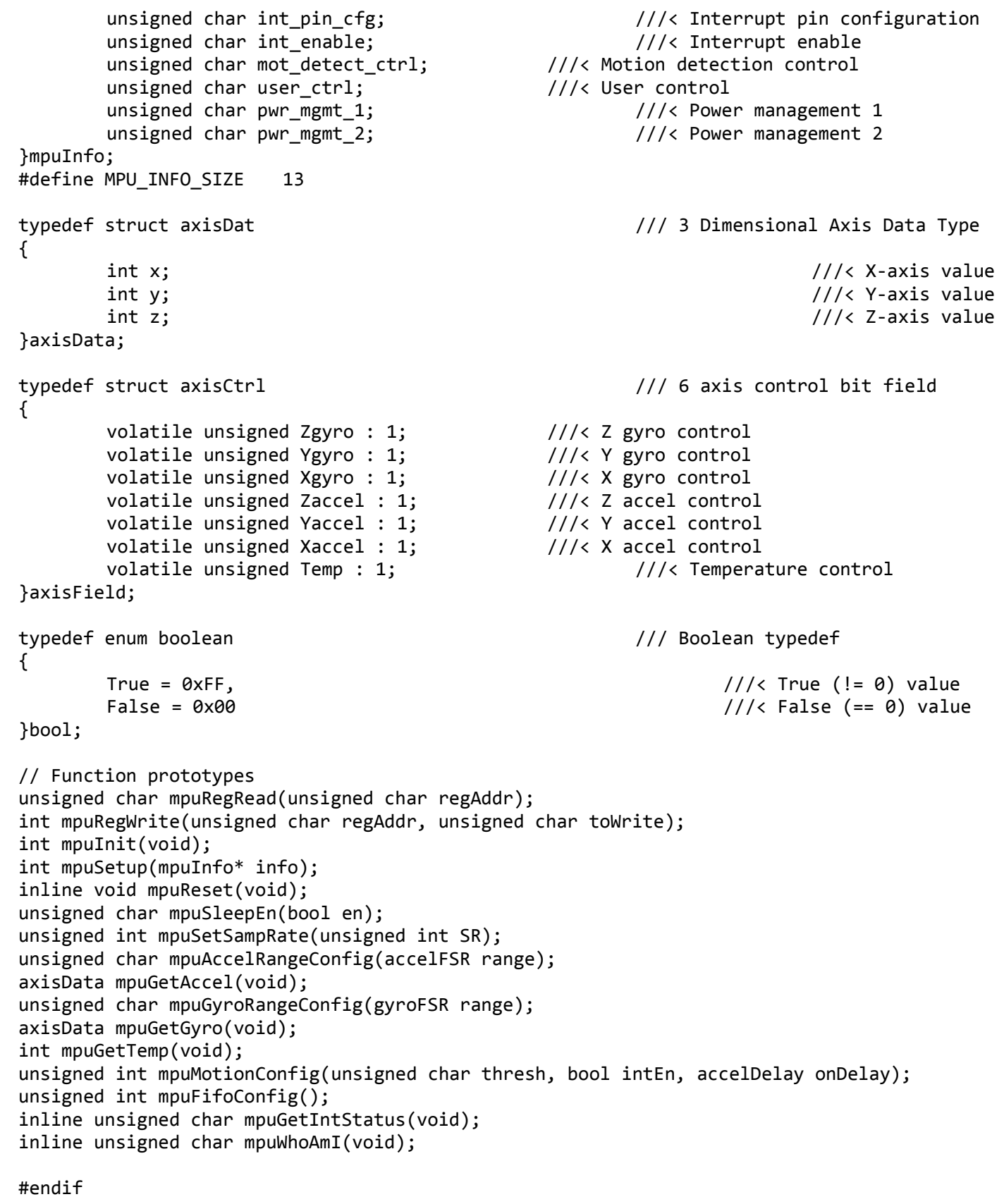

$/ / /<$ Interrupt enable

$/ / /<$ Motion detection control

$/ / /<$ User control

$/ / /<$ Power management 1

$/ / /<$ Power management 2

/// 3 Dimensional Axis Data Type

$/ / /<X$-axis value

$/ / /<Y$-axis value

$/ / /<Z$-axis value

/// 6 axis control bit field

$/ / /<\mathrm{Z}$ gyro control

$/ / /<Y$ gyro control

$/ / /<\mathrm{X}$ gyro control

$/ / /<\mathrm{Z}$ accel control

$/ / /<Y$ accel control

$/ / /<\mathrm{X}$ accel control

$/ / /<$ Temperature control

/// Boolean typedef

$$
/ / /<\operatorname{True}(!=0) \text { value }
$$$$
/ / /<\text { False }(==\theta) \text { value }
$$

\section{RTC.c}

Real-time clock module management library

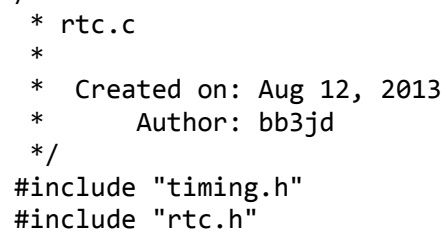




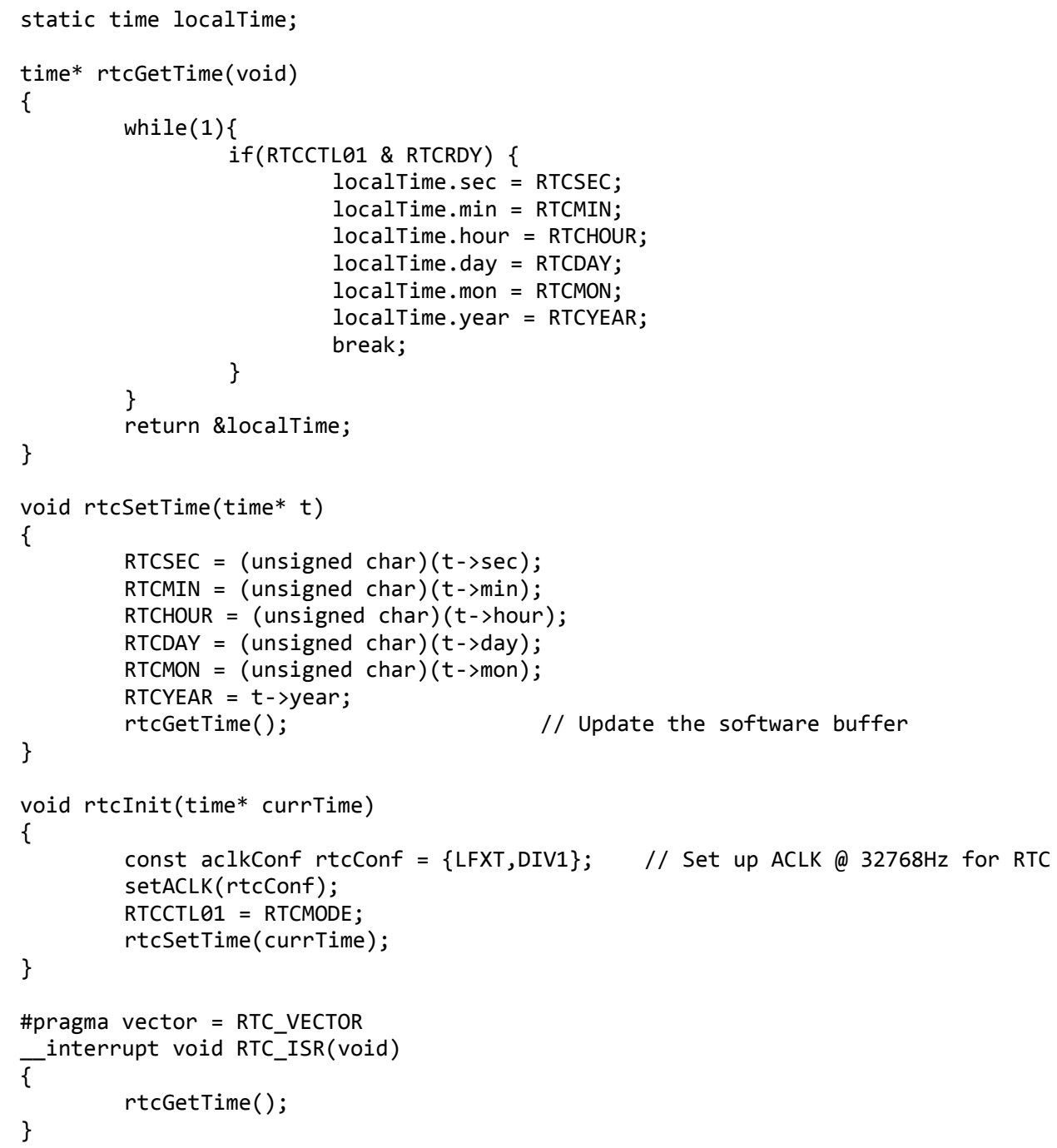

\section{RTC.h}

Affiliated real-time clock header file

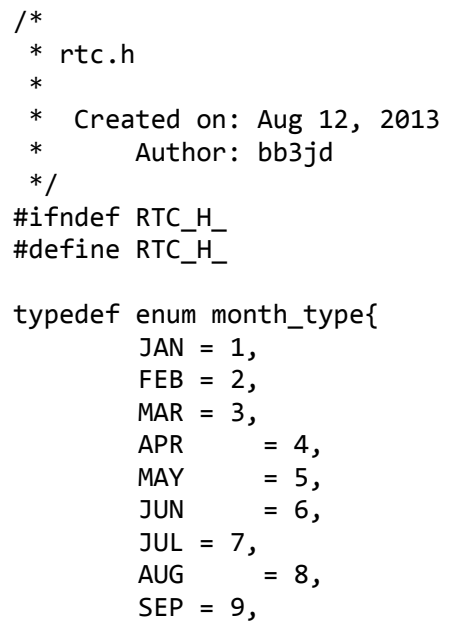




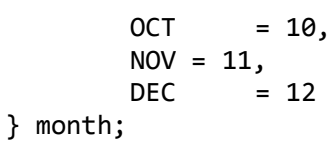

\section{Timing.c}

System timing and clock management library

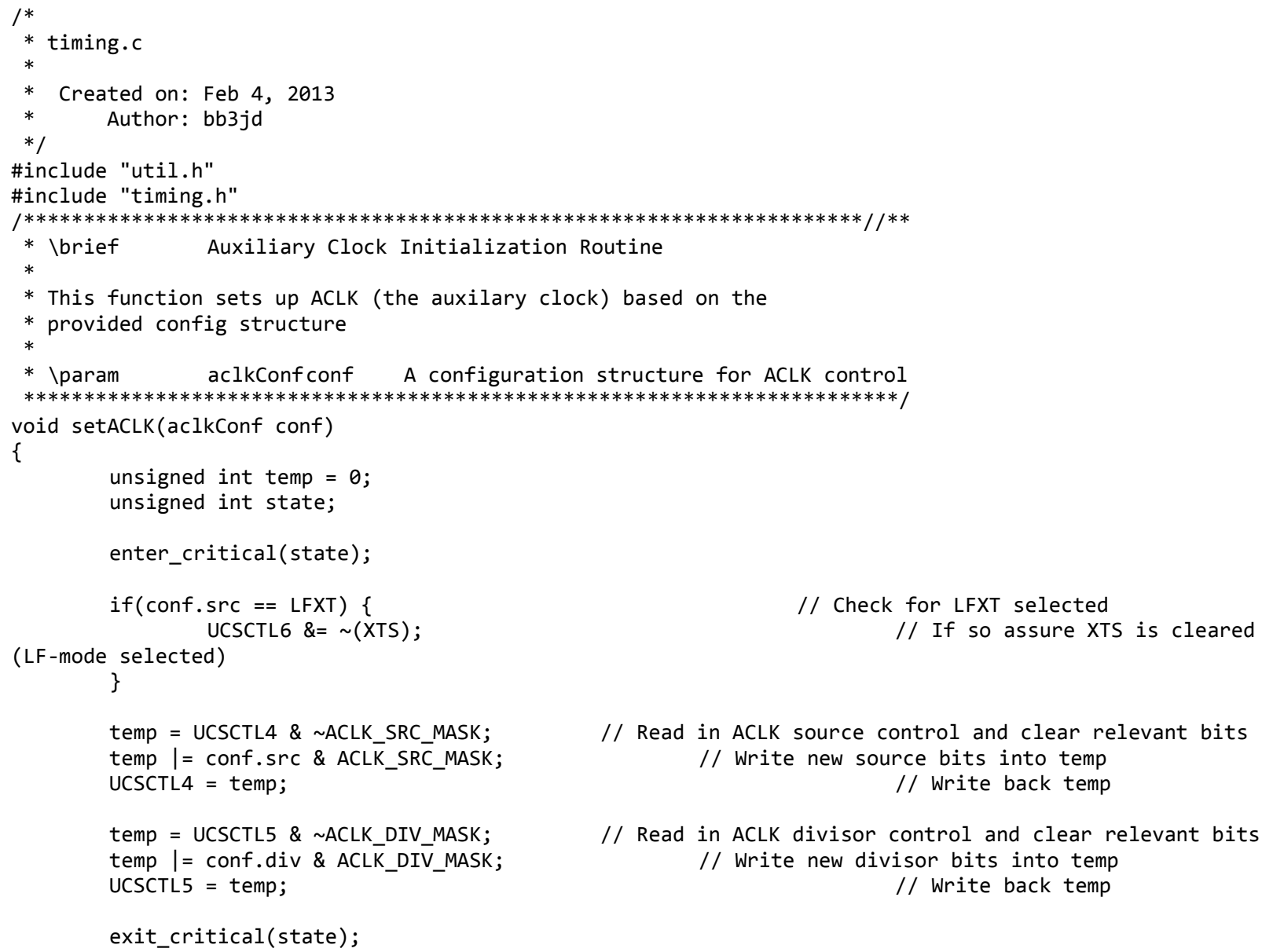




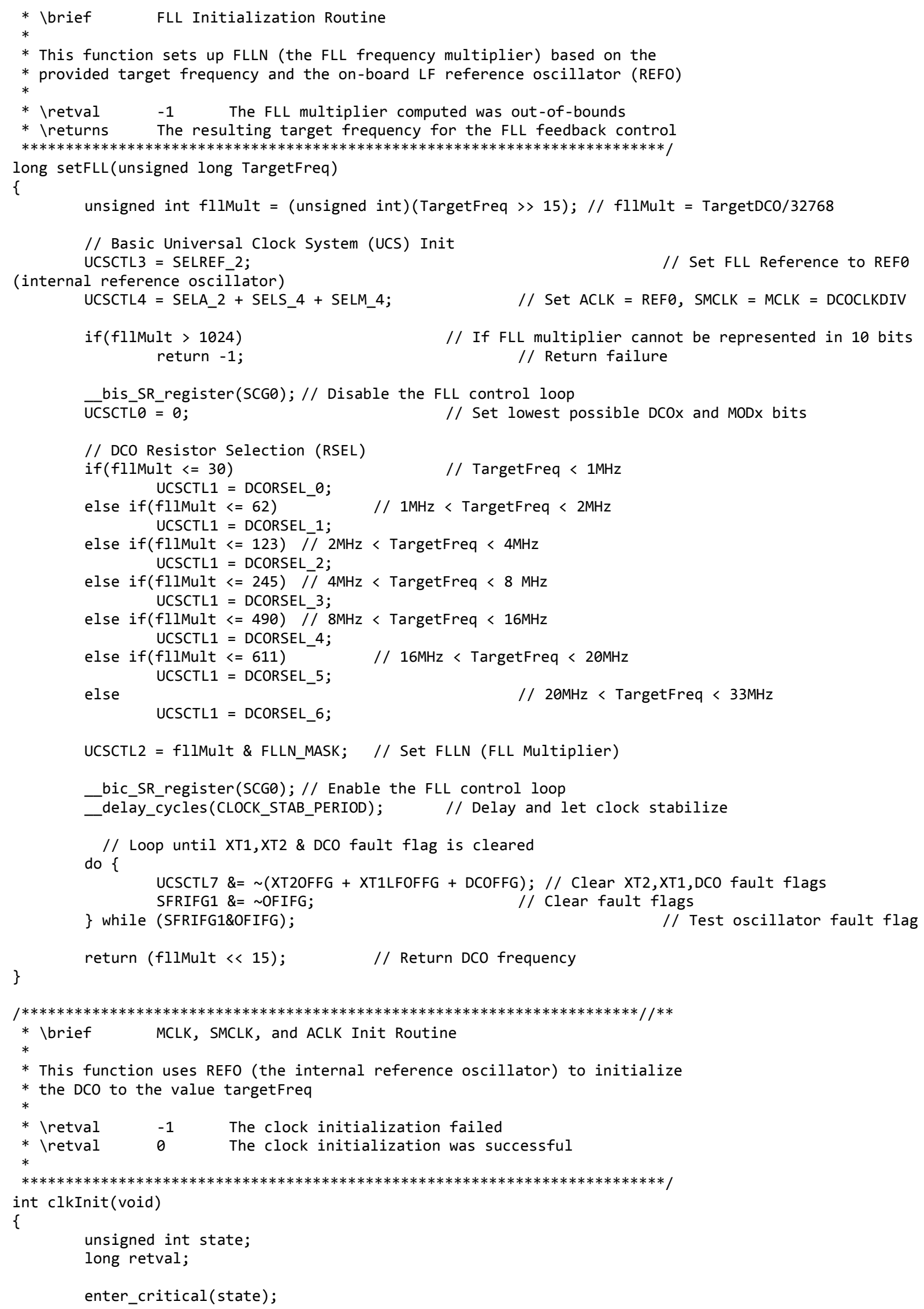




\section{Util.c}

Utilities for operation and useful functions

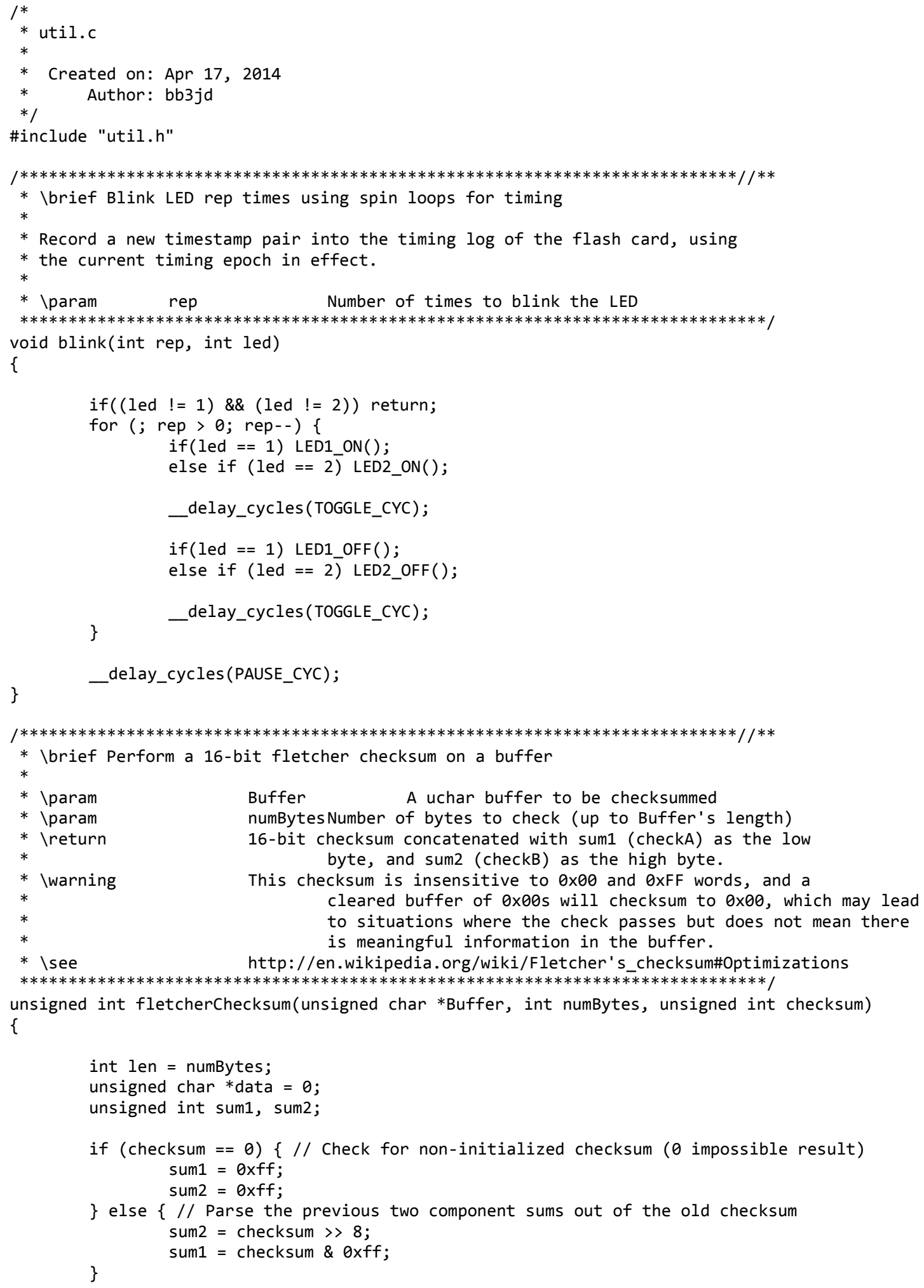




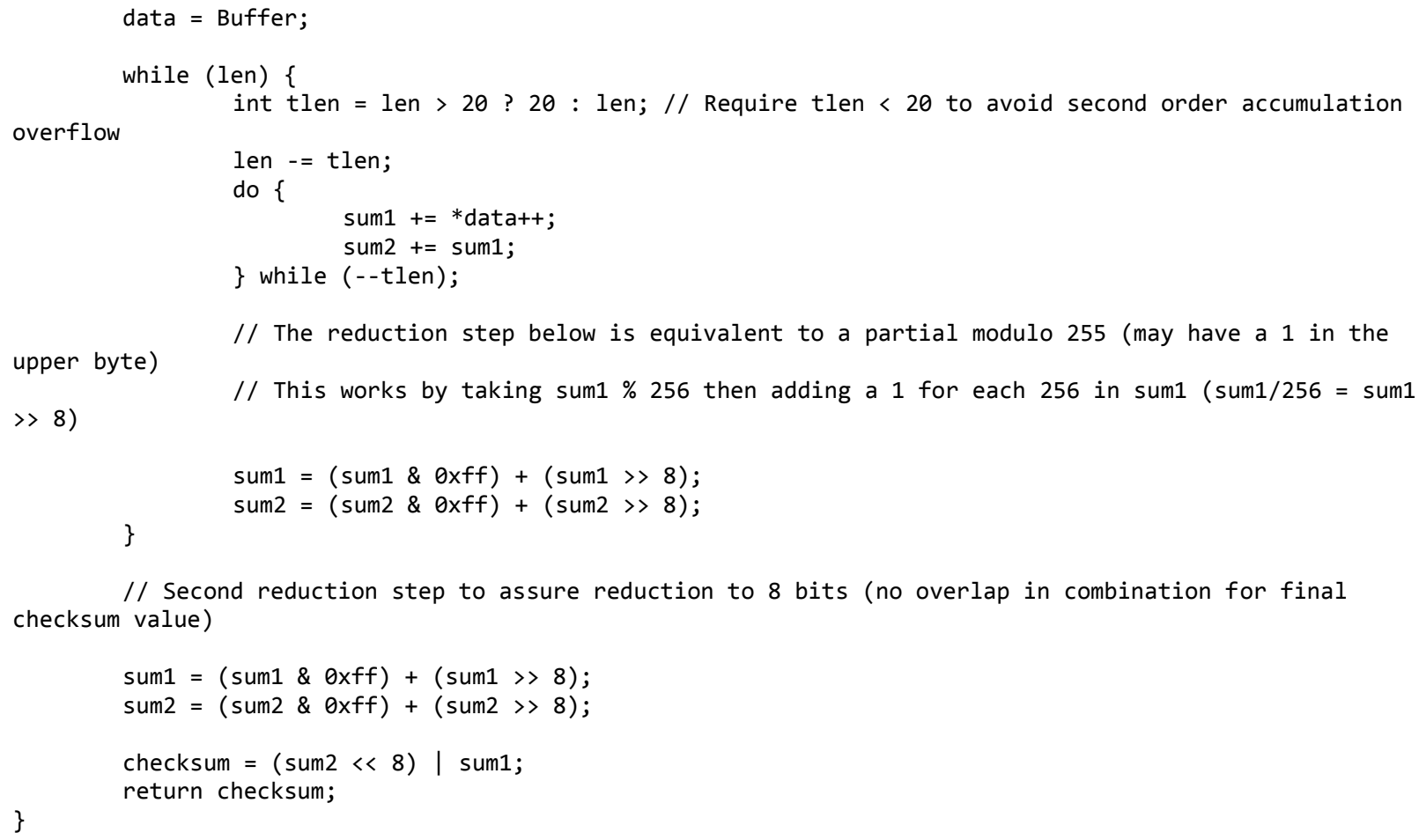

\section{Util.h}

Affiliated useful routine header file

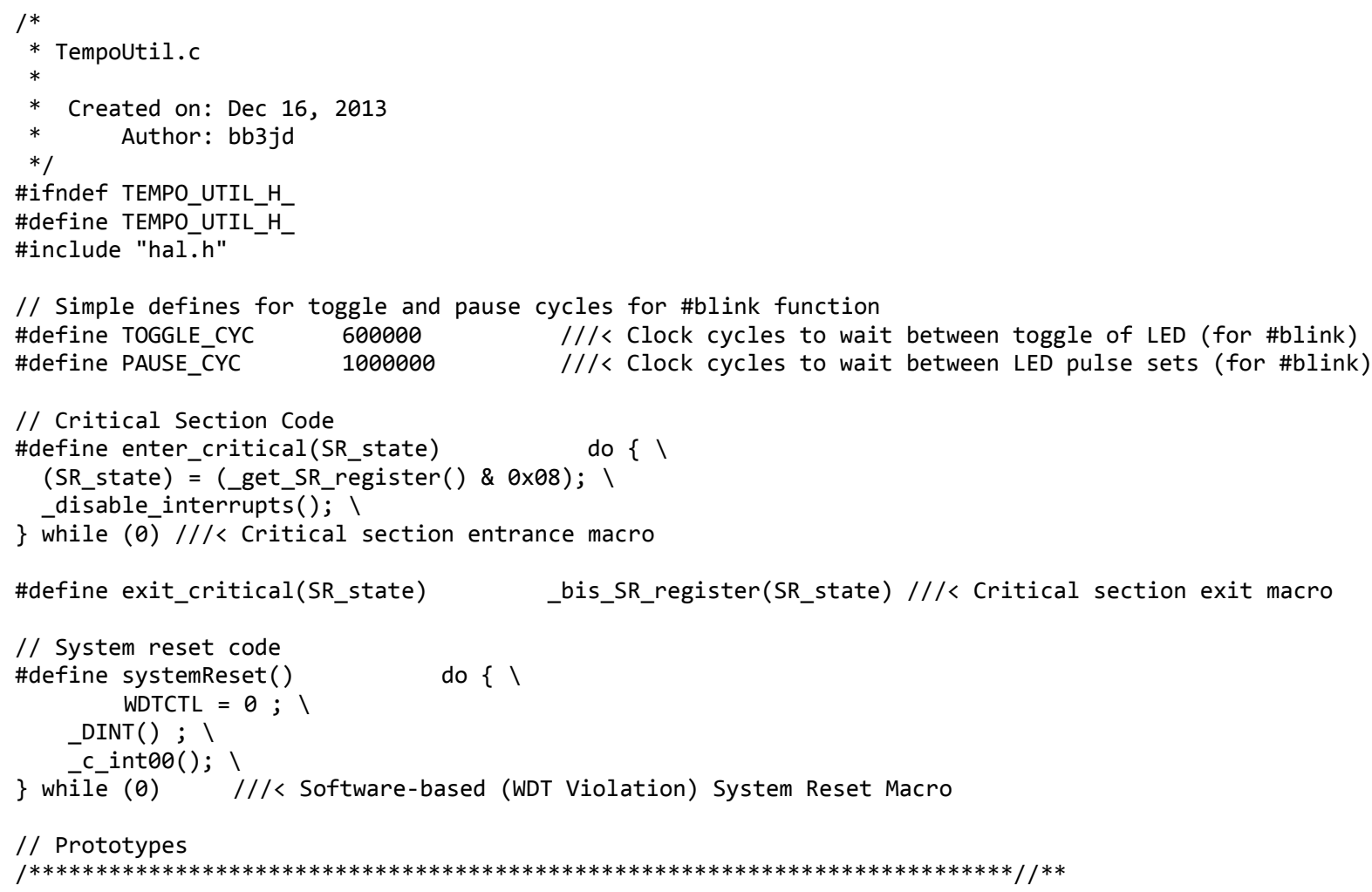


* Jbrief Blink LED rep times using spin loops for timing

$*$

* Record a new timestamp pair into the timing log of the flash card, using

* the current timing epoch in effect.

$*$

* Iparam rep Number of times to blink the LED

$* * * * * * * * * * * * * * * * * * * * * * * * * * * * * * * * * * * * * * * * * * * * * * * * * * * * * * * * * * * * * * * * * * * * * * * * * * * * *)$

void blink(int rep, int led);

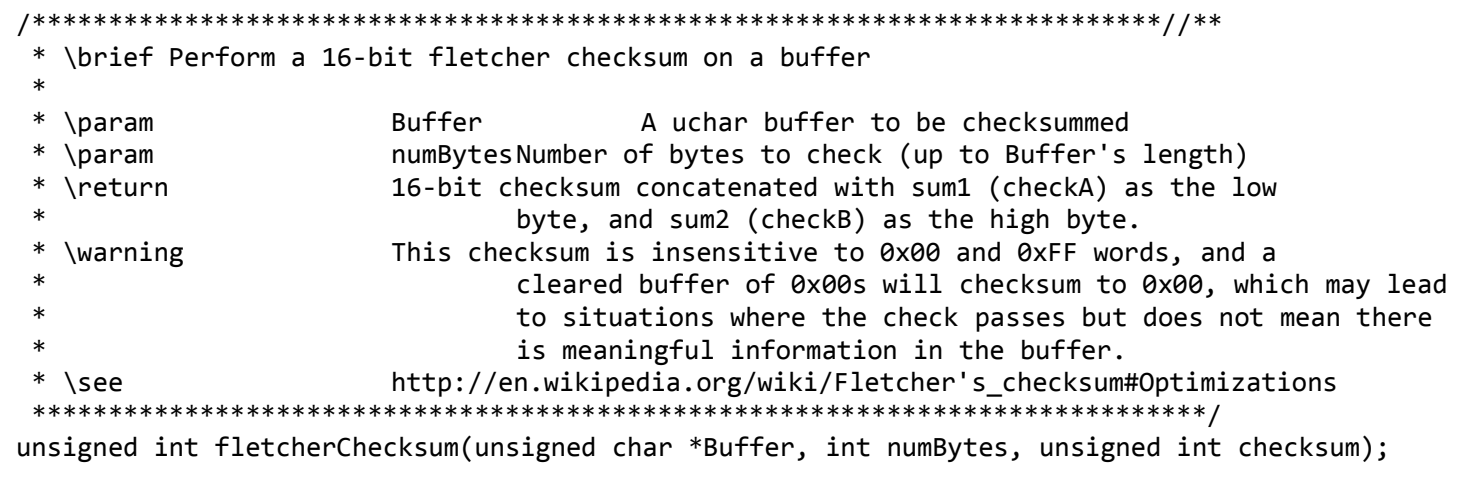

\#endif //TEMPO_UTIL_H_ 